\title{
A Learning Behaviour Based Controller for Maintaining Balance in Robotic Locomotion
}

by

Richard Beranek

A Dissertation submitted to

the Faculty of Graduate Studies and Postdoctoral Affairs

in partial fulfilment of

the requirements for the degree of

Doctor of Philosophy

in

Mechanical Engineering

Ottawa-Carleton Institute for

Mechanical and Aerospace Engineering

Department of Mechanical and Aerospace Engineering

Carleton University

Ottawa, Ontario, Canada

September 25, 2014

Copyright (c)

2014 - Richard Beranek 


\section{Abstract}

Maintaining balance in robotic systems has become an increasingly important control task as roboticists move towards designing systems, such as legged robots and mobile manipulators, to operate in unstructured environments. The balance problem is especially challenging for bipedal systems such as humanoid robots and exoskeletons. These systems have a small support polygon and a higher centre of mass (COM), making it more challenging to maintain balance. Although new control strategies have increased the robustness of bipeds to certain disturbances, they still lack the ability to walk on varied, uneven terrains, and to compensate for unknown disturbances.

The Behaviour-Based Locomotion Controller (BBLC) proposed in this thesis is a novel controller for robotic locomotion that introduces an architecture capable of generating new balancing strategies to compensate for unknown disturbances in the environment. The core feature of the BBLC is to apply the Behaviour-Based Control (BBC) architecture to the balance problem. In this architecture, several simpler control behaviours are combined together to generate more complex control strategies. In the BBLC, behaviours consist of different methods of planning task-space trajectories such as foot swing motions, torso motions and COM motions, in the case of a bipedal robot. A learning algorithm is then applied to determine which combination of behaviours result in successful balancing strategies for a given disturbance.

The BBLC is initially evaluated in simulation on two separate systems, a mobile manipulator and a planar biped. In both cases, the BBLC generates new balancing 
strategies which maintain balance when an unknown disturbance is applied. The BBLC is then implemented on ABL-BI (Advanced Biomechatronics and Locomotion Laboratory Biped One), a 13 degree of freedom bipedal robot designed to experimentally evaluate the BBLC. Initial learning of balancing strategies is performed in a 3D dynamic simulation of ABL-BI. The same disturbances were then applied to the experimental platform, where the results show that the learnt strategies from simulation were activated and increased the robustness of the system. Using a linear inverted pendulum model, it is shown the BBLC is capable of identifying a change in the stable region (in the COM phase plane) of the controller and select behaviours which can compensate for this change. These results confirm the BBLC generates new emergent balancing strategies capable of compensating for disturbances which are unknown to the controller. This controller architecture presents a novel framework capable of learning and adapting to a wide variety of disturbances, without needing a priori controller design for each disturbance case. 
Pro mamku a tatku. 


\section{Acknowledgments}

Completing this thesis has been a long but extremely rewarding journey that required the support of many people. First and foremost, I would like to thank Prof. Mojtaba Ahamadi. His role has been much more than thesis supervisor. Through the years, he has become a mentor and a close friend that has provided invaluable guidance in completing this work. A special thanks goes to all those who have contributed to the ABL-BI project throughout the years, most importantly Ali, Henry, and Owain; without your contributions this work would not have been possible. Working at the ABL laboratory has also provided a wonderful environment and I have made many valuable friendships.

I would like to thank my family which has continually provided support and encouragement. Manon, your love and support have been my foundation and have helped me through the most difficult times. Brett, you have taught me so much and always pushed me to achieve greater things. Lastly, I would like to thank my parents for their unconditional support. Before even my birth they started a daring journey to provide a better life for their family. I hope that this thesis, in some way, is a symbol showing that their journey has been successful. 


\section{Table of Contents}

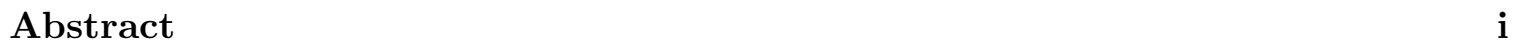

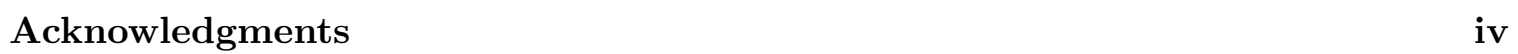

$\begin{array}{ll}\text { Table of Contents } & \mathrm{v}\end{array}$

List of Tables $\quad$ ix

List of Figures $\quad$ xi

List of Abbreviations $\quad x x$

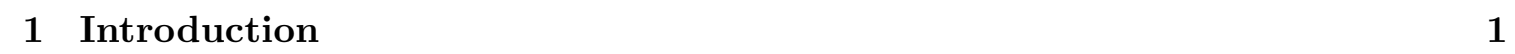

1.1 Motivation . . . . . . . . . . . . . . . . . . . . . 2

1.2 Objectives and Contributions $\ldots \ldots \ldots \ldots$

1.3 Thesis Overview $\ldots \ldots \ldots \ldots \ldots$

1.4 Background . . . . . . . . . . . . . . . . . . . 7

$1.4 .1 \quad$ Biped and Humanoid Robots . . . . . . . . . . . . . . . . . . . . . . 7

1.4 .2 Biped Locomotion Controllers . . . . . . . . . . . . . . . . . . . . 9

1.4 .3 Behaviour Based Control . . . . . . . . . . . . . . . . . . . 21

$\begin{array}{|ll|}2 & \text { Design of ABL-BI and Simulation Tools } \\ 24\end{array}$

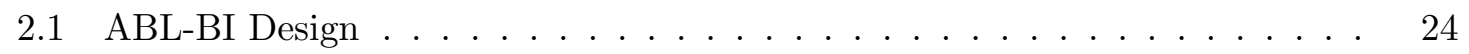

2.1 .1 Initial Requirements $\ldots \ldots \ldots \ldots$. . . . . . . . . . . . 25

$2.1 .2 \quad$ Mechatronic Design $\ldots \ldots \ldots \ldots \ldots \ldots$ 
2.1 .3 Communications and Control Architecture $\ldots \ldots \ldots \ldots$

$2.1 .4 \quad$ Software Architecture . . . . . . . . . . . . . . . . 32

$2.1 .5 \quad$ Kinematics and State Machine $\ldots \ldots \ldots \ldots$. . . . . . . 37

2.2 Simulation Tools . . . . . . . . . . . . . . . . . . . . . . . . 42

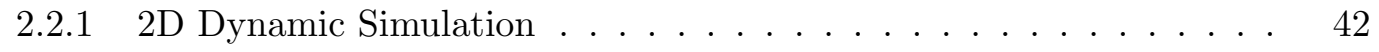

$2.2 .2 \quad 3$ D Dynamic Simulation $\ldots \ldots \ldots \ldots \ldots \ldots$

2.3 Chapter Summary $\ldots \ldots \ldots \ldots \ldots$

3 Behaviour-Based Locomotion Controller - Architecture and Implemen-

$\begin{array}{ll}\text { tation Examples } & 46\end{array}$

3.1 The Behaviour-Based Locomotion Controller - General Mathematical For-

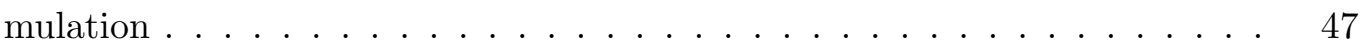

$3.1 .1 \quad$ Task layer $\ldots \ldots \ldots \ldots \ldots$

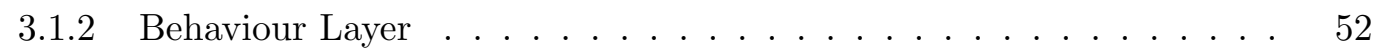

3.1 .3 Reinforcement Learning Layer $\ldots \ldots \ldots \ldots \ldots$

3.2 Mobile Manipulator Balance Control . . . . . . . . . . . . . . . . 57

$3.2 .1 \quad$ Task Layer $\ldots \ldots \ldots$

$3.2 .2 \quad$ Behaviour Layer $\ldots \ldots \ldots \ldots \ldots$

3.2 .3 Reinforcement Learning Layer $\ldots \ldots \ldots \ldots$

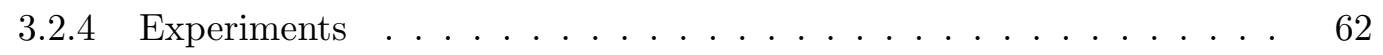

$3.3 \quad$ Planar Biped Balance Control $\ldots \ldots \ldots \ldots$. . . . . . . . . . . . 68

3.3 .1 Task Layer $\ldots \ldots \ldots \ldots$

$3.3 .2 \quad$ Behaviour Layer $\ldots \ldots \ldots \ldots \ldots$

$3.3 .3 \quad$ Behaviour Learning Algorithm $\ldots \ldots \ldots \ldots$

3.3 .4 Experiments . . . . . . . . . . . . . . . . . . 71

3.4 Chapter Summary $\ldots \ldots \ldots \ldots \ldots \ldots \ldots$

$\begin{array}{|lll|}4 & \text { BBLC Implementation on ABL-BI: Simulation } & 80\end{array}$

$4.1 \quad$ BBLC for ABL-BI $\ldots \ldots \ldots \ldots \ldots$

4.1 .1 Task Layer $\ldots \ldots \ldots \ldots$ 
$4.1 .2 \quad$ Behaviour Layer $\ldots \ldots \ldots$. . . . . . . . . . . . . . . . . . . 82

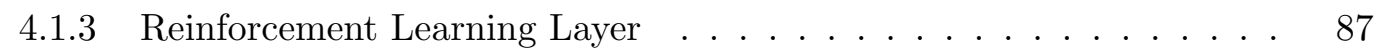

4.2 Simulation Results $\ldots \ldots \ldots \ldots$

4.2 .1 Walking . . . . . . . . . . . . . . . . . . . . . . . 90

$4.2 .2 \quad$ Push Disturbance $\ldots \ldots \ldots \ldots$

4.2 .3 Step Disturbance $\ldots \ldots \ldots$. . . . . . . . . . . . . . . 102

$4.2 .4 \quad$ Slope Disturbance $\ldots \ldots \ldots$. . . . . . . . . . . . . . 106

4.3 Chapter Summary . . . . . . . . . . . . . . . . . . . 107

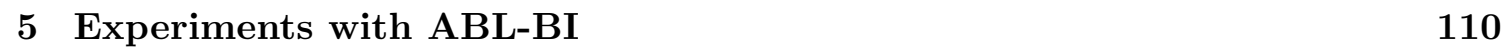

5.1 Estimation of Ground Reaction Forces . . . . . . . . . . . . . . . . . . . . . 111

$5.2 \quad$ BBLC Experiments on ABL-BI and Analysis . . . . . . . . . . . . . . . 114

$5.2 .1 \quad$ Periodic Walking Experiment . . . . . . . . . . . . . . . . . 115

$5.2 .2 \quad$ Push Disturbance Experiment $\ldots \ldots \ldots \ldots$

$5.2 .3 \quad$ Step Disturbance Experiment . . . . . . . . . . . . . . . . . . . . . 124

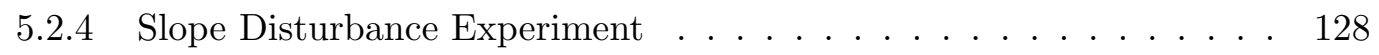

5.3 Chapter Summary $\ldots \ldots \ldots \ldots$. . . . . . . . . . . . . . . . . 132

6 Stability Analysis of Behaviour Strategies Using the Linear Inverted $\begin{array}{ll}\text { Pendulum Model } & 134\end{array}$

$6.1 \quad$ LIPM Stability Analysis $\ldots \ldots \ldots \ldots$. . . . . . . . . . . . . . . . 134

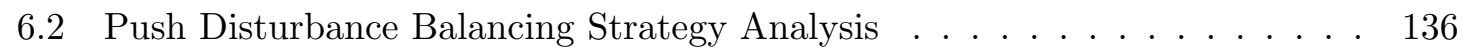

$6.2 .1 \quad$ CMP Controller Effect on Stability . . . . . . . . . . . . . . . 137

$6.2 .2 \quad$ Effect of Excluding Swing Leg Motion . . . . . . . . . . . . . . . . . 139

6.2 .3 Results $\ldots \ldots \ldots \ldots \ldots \ldots$

6.3 Chapter Summary . . . . . . . . . . . . . . . . . . 145

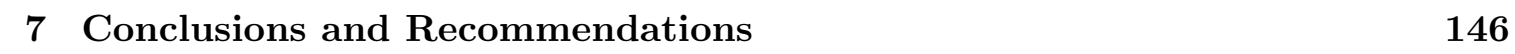

7.1 Conclusions . . . . . . . . . . . . . . . . . . . . . . . . . 146

$7.1 .1 \quad$ BBLC Architecture $\ldots \ldots \ldots \ldots$. . . . . . . . . . . 146 
7.1 .2 Simulation and Learning . . . . . . . . . . . . . . . . . . . . 147

$7.1 .3 \quad$ Experimental Validation $\ldots \ldots \ldots$. . . . . . . . . . 147

7.2 Recommendations for Future Work . . . . . . . . . . . . . . . . . . . . . . . 148

\begin{tabular}{ll}
\hline List of References & 150
\end{tabular}

Appendix A Transformation Equations and Parameters for ABL-BI Kine-

matics

163

\begin{tabular}{lll}
\hline Appendix B Calibration Curves for Ground Reaction Force Estimator & 168
\end{tabular} 


\section{List of Tables}

$2.1 \quad$ Design requirements for the ABL-BI robot. . . . . . . . . . . . . . . . 26

2.2 Estimated peak and average torques for each joint. . . . . . . . . . . . . . 28

2.3 Selected actuation system for the ABL-BI design based on estimated torque requirements. . . . . . . . . . . . . . . . . . 30

2.4 Joint definitions and rotation axis. . . . . . . . . . . . . . . . . 40

2.5 Biped model specifications. $\ldots \ldots \ldots \ldots \ldots$. . . . . . . . . . . 42

$2.6 \quad$ Ground modelling parameters for planar biped simulation. . . . . . . . . . . 44

$3.1 \quad$ Mobile manipulator model parameters. . . . . . . . . . . . . . . . . . . 58

3.2 Behaviours for mobile manipulator. . . . . . . . . . . . . . 60

$3.3 \quad$ Behaviours for planar biped implementation . . . . . . . . . . . 70

3.4 Gait parameters. . . . . . . . . . . . . . . . . . . . 71

3.5 Reinforcement learning algorithm parameters. . . . . . . . . . . . 75

$3.6 \quad$ Reinforcement learning performance for bipedal robot simulation. . . . . . . 76

4.1 Summary of behaviours for each task-space motion. . . . . . . . . . . 83

$4.2 \quad$ Discretization of the reduced state-space for the Q-value lookup table. . . . 88

$4.3 \quad$ Values of learning parameters used in the reinforcement learning algorithm. 89

4.4 Gait parameters used to plan walking motion of ABL-BI. . . . . . . . . . . 91

4.5 Summary of simulation results for 4 different cases that were examined. . . 108

$5.1 \quad$ Calibrated GRF estimator parameters. . . . . . . . . . . . . . . . . . . . 113

5.2 Gait parameters used for walking experiment. . . . . . . . . . . . . 115

$5.3 \quad$ Comparison of state vector initiating balancing strategy between experiment and simulation for the push disturbance experiment. . . . . . . . . . . . 120 
5.4 Comparison of the state vector for which a balancing strategy is activated in the simulated and experimental step walking experiments. . . . . . . . . . 125

5.5 Comparison of the state vectors for which a balancing strategy is activated in the simulated and experimental slope walking experiments. . . . . . . . . 129

A.1 Relative position between joint frames. . . . . . . . . . . . . . . . . . 163

A.2 Relative position of the joint centre of mass to joint frame. . . . . . . . . . 164 


\section{List of Figures}

1.1 Humanoid robots designed in industry (a)ASIMO by Honda corporation [1] (b) HOAP-3 by Fujitsu 2 , (c) QRIO by Sony $[3] . \ldots \ldots$. . . . . . . . . . 7

$1.2 \quad$ Research biped platforms: (a) Rabbit $|4|$ (b) JOHNNIE $[5]$. . . . . . . . . 8

$1.3 \quad$ Linear inverted pendulum model for humanoid robots $|6| . \ldots$. . . . . . . 11

1.4 (a) Spring flamingo robot with series elastic actuators $[7$, , (b) virtual spring damper forces are applied to the biped to stabilize it [7] . . . . . . . 15

1.5 Reinforcement learning block diagram. . . . . . . . . . . . . . . 17

1.6 The location of the ground reference points CMP, ZMP, and COM when there is (a) an uncompensated moment acting about the COM, and (b) zero moment acting about the COM. . . . . . . . . . . . . . 20

1.7 Behaviour based control architecture . . . . . . . . . . . . . . . . . . . . . 21

1.8 Coordination architectures for behaviour-based systems; (a) competitive, (b) cooperative. . . . . . . . . . . . . . . . . . . 22

$2.1 \quad$ Different conceptual designs initially developed for ABL-BI: (a) DC gearmotor actuation concept with pulley transmission, (b) DC gearmotor actuation concept using pulley transmissions and a linear actuator for the hip pitch joint and (c) using direct actuation of each joint using DC gearmotors. . . . 27

2.2 Graphical representation of the initial dynamic models used for actuator sizing and concept evaluation using Webots ${ }^{\mathrm{TM}}$ software. . . . . . . . . . 28

2.3 Different stances that were used to evaluate required joint torques: (a) maximum side weight shift, (b) maximum forward weight shift, and (c) crouched position. . . . . . . . . . . . . . . . . . 29 
2.4 Example of resulting applied torque on the right hip roll joint to move the robot to the maximum side weight shift position (Figure $2.3(\mathrm{a})$ ). . . . . . . 29

2.5 Progression of ABL-BI design from concept to detailed mechanical design and completed assembly. . . . . . . . . . . . . . . . . . . . 30

$2.6 \quad$ ABL-BI with associated joint numbers as defined in Table 2.4 . . . . . . . 31

2.7 Communication and control architecture for ABL-BI. Control is implemented on a Cheetah EMP-32 embedded PC which communicates velocity commands via two CAN busses to Elmo Whistle motor drives. The drives implement a local velocity control loop with encoder feedback from motors (Maxon RE-30 or RE-40) and implement joint limits locally. . . . . . . . . . . . . . . 33

2.8 Software layers in ABL-BI control architecture. . . . . . . . . . . . . . . . . 34

$2.9 \quad$ Architecture for individual software layers. . . . . . . . . . . . . . 36

2.10 The different states of walking used in locomotion planning and control. . . $\quad 38$

2.11 Kinematic configuration of the ABL-BI robot. . . . . . . . . . . . . . . 40

2.12 2D planar biped model. . . . . . . . . . . . . . . . . . . . . 43

2.13 Visualization of the 3D dynamic simulation of ABL-BI. . . . . . . . . . . 44

$3.1 \quad$ The BBLC architecture $\ldots \ldots \ldots \ldots \ldots$. . . . . . . . . . . . . . . 48

3.2 The task layer combines the output for each task-space controller, with priority defined from the behaviour layer, to compute the final set of reference joint angles. . . . . . . . . . . . . . . . . . . . . . . . 49

3.3 The behaviour layer has multiple locomotion behaviours which independently generate a reference task-space trajectory for each task-space motion. For each task-space motion, the trajectory from one locomotion behaviour is selected. A learning algorithm is used to determine which behaviour combinations lead to stabilization in the presence of disturbances. . . . . . . . . . 53

$3.4 \quad$ Flow chart describing the learning process as it occurs in simulation. . . . . $\quad 56$

3.5 Mobile-manipulator dynamic model is composed of a four wheel, car like steering mobile base and a five DOF manipulator. . . . . . . . . . . . 58 
3.6 Mobile-manipulator recovering from a potential fall with $85 \mathrm{~kg}$ load at the end-effector. . . . . . . . . . . . . . . . . . . . 64

$3.7 \quad$ Q-function for the reference behaviour combination where the end-effector moves to a user defined position. Negative Q-values are accumulated when this behaviour combination is unable to compensate for tipping caused by a large load at the end-effector. . . . . . . . . . . . . . . . 64

3.8 Q-function for the behaviour combination preventing tip over, when an 85 $\mathrm{kg}$ load is placed at the end-effector. . . . . . . . . . . . . . . 65

3.9 Mobile-manipulator tipping over on a $40^{\circ}$ slope. . . . . . . . . . . . . . . . 66

3.10 Q-function for the behaviour combination used for reference trajectory tracking. 66

3.11 Q-function for the behaviour combination resulting in a successful stabilization strategy on sloped surfaces. $\ldots \ldots \ldots \ldots$. . . . . . . . . 67

3.12 Mobile-manipulator recovering on a $40^{\circ}$ slope. . . . . . . . . . . . . . . 67

3.13 Q-function for the behaviour combination used for stable walking on flat terrain. Negative Q-values are accumulated when this behaviour combination is unable to compensate for push-type disturbances. . . . . . . . . . . . . 73

3.14 Biped motion after push disturbance on a flat terrain. In this motion the biped shifts the COM backwards to counter the effect of the disturbance. . $\quad 73$

3.15 Q-function for a behaviour combination capable of stabilizing the robot to push-type disturbances. . . . . . . . . . . . . . . . . . . 74

3.16 Learning curve for biped robot push test on flat terrain. A successful behaviour combination is found after 42 episodes. . . . . . . . . . . . 76

3.17 Q-function for reference walking behaviour combination on sloped terrain. . $\quad 77$

3.18 Q-function for the behaviour combination capable of stabilizing the robot when a push-type disturbance is applied on a slope. . . . . . . . . . . . 77

3.19 Biped motion after a push disturbance on a $10^{\circ}$ slope. . . . . . . . . . . . 78

4.1 The method of estimating a slope angle is illustrated. The height difference at landing between the front and rear of the foot are used to estimate the ground slope angle $\theta . \ldots \ldots \ldots \ldots \ldots$. . . . . . . . . . . . . 84 
4.2 Reference ZMP or CMP trajectories for the $\mathrm{X}$ and $\mathrm{Z}$ axes for a cycle of walking. The figure is divided between different walking stances as defined in Chapter $2(\mathrm{WS}=1$ double stance left foot, $\mathrm{WS}=3$ single stance left foot, $\mathrm{WS}=4$ double stance right foot, $\mathrm{WS}=6$ single stance right foot). Note a step occurs between $\mathrm{WS}=3$ and $\mathrm{WS}=4$ due to a change in reference frames. . . . 85

4.3 Freeze frame view of the biped during walking. (a) Double stance start; (b) weight shift to left foot; (c) right foot swing; (d) right foot down - double stance; (e) weight shift to right foot; (f) left foot swing. . . . . . . . . . . .

4.4 The phase plane of the X COM motion is shown in (a) and corresponding Poincaré map in (b) for a 17 step walking simulation. . . . . . . . . . . . . 92

4.5 The phase plane of the Y COM motion is shown in (a) and corresponding Poincaré map in (b) for a 17 step walking simulation. . . . . . . . . . . . 93

4.6 The phase plane of the Z COM motion is shown in (a) and corresponding Poincaré map in (b) for a 17 step walking simulation. . . . . . . . . . . . 94

4.7 The balancing strategy being used in the BBLC controller where strategy 2 corresponds to an activation vector of $\vec{A}=\left[\begin{array}{llllll}1 & 3 & 2 & 3 & 2 & 1\end{array}\right]^{T}$ and strategy 1 to $\vec{A}=\left[\begin{array}{lllll}1 & 1 & 1 & 1 & 1\end{array}\right]^{T}$. The strategy is activated at $6.752 \mathrm{~s}$. The magnitude of the disturbance is $15 \mathrm{~N}$ in the $\mathrm{X}$ axis and $25 \mathrm{~N}$ in the $\mathrm{Z}$ axis (red area corresponds to the period when the disturbance is applied). . . . . . . . . . . 96

4.8 Motion of ABL-BI when a push is continually applied with a magnitude of $15 \mathrm{~N}$ in the $\mathrm{X}$ axis and $25 \mathrm{~N}$ in the $\mathrm{Z}$ axis. The initial position is shown in yellow, while the final steady state position is in grey. The motion of ABL-BI is against the direction of the disturbance. . . . . . . . . . . . . . 97

4.9 As a result of the push-type disturbance (red area corresponds to the period when the disturbance is applied) the CMP behaviour is activated for planning the COM motion. The resulting motion for X COM is shown in (a) where the dotted boundary denotes the support polygon and the measured GRF in the $\mathrm{X}$ axis in (b) . . . . . . . . . . . . . . . . . . 97 
4.10 As a result of the push-type disturbance (red area corresponds to the period when the disturbance is applied) the CMP behaviour is activated for planning the COM motion. The resulting motion for $\mathrm{Z} \mathrm{COM}$ is shown in (a) where the dotted boundary denotes the support polygon and the measured GRF in the $\mathrm{Z}$ axis in $(\mathrm{b}) . \ldots \ldots \ldots \ldots \ldots$

4.11 The balancing strategy being used in the BBLC controller where strategy 2 corresponds to an activation vector of $\vec{A}=\left[\begin{array}{llllll}1 & 3 & 2 & 3 & 2 & 1\end{array}\right]^{T}$ and strategy 1 to $\vec{A}=\left[\begin{array}{llllll}1 & 1 & 1 & 1 & 1 & 1\end{array}\right]^{T}$. The strategy is activated at $6.804 \mathrm{~s}$ and the magnitude of the disturbance (red area corresponds to the period when the disturbance is applied) is $10 \mathrm{~N}$ in the $\mathrm{X}$ axis and $20 \mathrm{~N}$ in the $\mathrm{Z}$ axis. . . . . . . . . . . 99

4.12 The area of push magnitudes under which a balancing strategy $(\vec{A}=$ $\left.\left[\begin{array}{llllll}1 & 3 & 2 & 3 & 2 & 1\end{array}\right]^{T}\right)$ is activated to maintain balance, given one learnt case. . . . . 100

4.13 Surface plot showing Q-values as a function of the measured GRFs. All other states are held constant for this plot at values of $0^{\circ}$ for the slope estimate, Walking State $=3$, and stability margin $<0.01 \mathrm{~m}$. . . . . . . . . . . . 101

4.14 Balancing strategy activation for a transient disturbance (red area corresponds to the period when the disturbance is applied). The balancing strategy is activated $0.752 \mathrm{~s}$ after the disturbance is applied and deactivates 0.3050 s after the disturbance is removed. . . . . . . . . . . . . . . . . . 102

4.15 Walking motion of ABL-BI when a $1 \mathrm{~cm}$ step is placed under the right foot: (a) DBL; (b) DBLS; (c) SSL; (d) DBR. . . . . . . . . . . . . . . . . 103

4.16 Activation of the balancing strategy when walking with a $1 \mathrm{~cm}$ step under the right foot in (b). The balancing strategy activation coincides with the robot being in single stance on the right foot where the walking state is shown in (a).103

4.17 Balancing strategy is artificially kept activated in (a) causing a continual drop in the Y COM position in (b), eventually resulting in a fall. . . . . . . 105

4.18 Motion of ABL-BI when walking up a $5^{\circ}$ slope: (a) double stance; (b) left foot swing; (c) double stance; (d) right foot swing; (e) double stance. . . . . 105 
4.19 Balancing strategy activation when walking on a $5^{\circ}$ slope. The balancing strategy activation coincides with single stance for both the right and left foot.106

4.20 The slope estimate for a complete walking cycle where the robot transitions from a flat to sloped terrain. . . . . . . . . . . . . . . . . . . 107

5.1 Comparison of the measured and estimated GRFs from simulation for both the $\mathrm{X}$ axis in (a) and $\mathrm{Z}$ axis in (b). The applied disturbance is $15 \mathrm{~N}$ in the $\mathrm{X}$ axis and $25 \mathrm{~N}$ in the $\mathrm{Z}$ axis. . . . . . . . . . . . . . . . . . . . . . . 113

5.2 Method for calibrating lateral GRF estimation. A known mass is suspended generating a disturbance of a known magnitude acting on ABL-BI. . . . . . 114

5.3 Resulting motion of ABL-BI during a balanced cyclic walk. Each frame corresponds to the following walking states: (a) DBL, (b) DBLS, (c) SSL, (d) DBR, (e) DBRS, (f) SSR, (g) DBL, (h) DBL. . . . . . . . . . . . . . . . 116

5.4 The phase plane of the X COM motion is shown in (a) and corresponding Poincaré map in (b) for 8 steps of the walking experiment. . . . . . . . . . . 117

5.5 The phase plane of the Y COM motion is shown in (a) and corresponding Poincaré map in (b) for 8 steps of the walking experiment. . . . . . . . . . . 117

5.6 The phase plane of the Z COM motion is shown in (a) and corresponding Poincaré map in (b) for 8 steps of the walking experiment. . . . . . . . . . . 118

5.7 Resulting motion of ABL-BI when a push disturbance is applied to the pelvis during SSL. Frame (a) corresponds to the initial posture before the disturbance is applied, (b) the posture after the disturbance is applied and (c) a comparison of the intail and final postures, where the shaded area corresponds to the initial posture. . . . . . . . . . . . . . . . . . . . . . 119

5.8 The balancing strategy being used in the BBLC controller where strategy 2 corresponds to an activation vector of $\vec{A}=\left[\begin{array}{llllll}1 & 3 & 2 & 3 & 2 & 1\end{array}\right]^{T}$. The strategy is activated at $12.52 \mathrm{~s}$ and the magnitude of the disturbance is $15 \mathrm{~N}$ in the $\mathrm{X}$ axis and $23 \mathrm{~N}$ in the $\mathrm{Z}$ axis (red area corresponds to the period when the disturbance is applied) 
5.9 As a result of the push disturbance (red area corresponds to the period when the disturbance is applied) the CMP behaviour is activated for planning the COM motion. The resulting motion for X COM is shown in (a) and the estimated GRF in the X axis in (b). . . . . . . . . . . . . . . . . . . . . 121

5.10 As a result of the push-type disturbance (red area corresponds to the period when the disturbance is applied) the CMP behaviour is activated for planning the COM motion. The resulting motion for X COM is shown in (a) where the dotted boundary denotes the support polygon and the measured GRF in the $\mathrm{X}$ axis in (b) . . . . . . . . . . . . . . . . . . . . . . . 122

5.11 As a result of the push disturbance (red area corresponds to the period when the disturbance is applied) the CMP behaviour is activated for planning the COM motion. The resulting motion for Z COM is shown in (a) and the estimated GRF in the $\mathrm{Z}$ axis in (b). . . . . . . . . . . . . . . . . . . . 122

5.12 As a result of the push-type disturbance (red area corresponds to the period when the disturbance is applied) the CMP behaviour is activated for planning the COM motion. The resulting motion for Z COM is shown in (a) where the dotted boundary denotes the support polygon and the measured GRF in the $\mathrm{Z}$ axis in (b). . . . . . . . . . . . . . . . . . . . . 123

5.13 The set of different push disturbance tests resulting in a balanced posture as a function of the magnitude of the disturbance. The dotted box corresponds to the boundary within which the robot remains balanced while applying the default action vector. . . . . . . . . . . . . . . . . . . . . . . . . 124

5.14 Resulting motion of ABL-BI during the step walk test. Each frame corresponds to the following walking states: (a) DBR, (b) DBRS, (c) SSR, (d) DBL, (e) DBLS, (f) SSL, (g) DBR, (h) DBR. . . . . . . . . . . . . . . . 126

5.15 Activation of the balancing strategy when walking with a $1 \mathrm{~cm}$ step under the right foot in (b). The balancing strategy activation coincides with the robot being in single stance on the right foot (walking state 6) where the

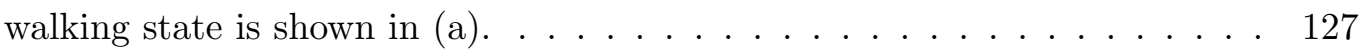


5.16 The phase plane of the X COM motion is shown in (a) and corresponding Poincaré map in (b) for 12 steps of the step walking experiment. . . . . . . 127

5.17 The $5^{\circ}$ slope along which ABL-BI walks during the slope experiment. . . . 129

5.18 Resulting motion of ABL-BI during the step walk test. Each frame corre\begin{tabular}{|c|}
\hline sponds to the following walking states: (a) DBL, (b) DBLS, (c) SSL, (d) \\
\hline
\end{tabular} DBR, (e) DBRS, (f) SSR, (g) DBL, (h) DBL. . . . . . . . . . . . . . . . 130

5.19 Balancing strategy activation when walking on a $5^{\circ}$ slope shown in (b). The balancing strategy activation coincides with single stance for both the right and left foot, where the walking state is shown in (a). . . . . . . . . . 131

5.20 The phase plane of the X COM motion is shown in (a) and corresponding Poincaré map in (b) for a 10 steps of the slope walking experiment. . . . . . 131

6.1 Stable region restriction on the X COM phase plane where the green region is stable and red is unstable. . . . . . . . . . . . . . . . . . . 137

6.2 Stable region restriction on the X COM phase plane where the green region is stable and the yellow region corresponds to the undisturbed stable region. 139

6.3 Maximum achievable COM velocity during push disturbance test as estimated by kinematic manipulability for (a) X COM and (b) Z COM. The manipulability is reduced during single stance, but returns to a higher level

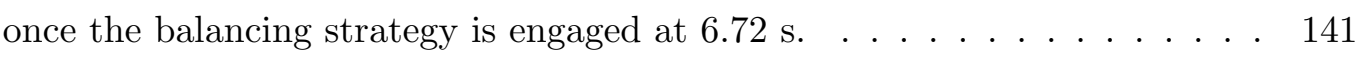

6.4 Stability region for X COM phase plane under $15 \mathrm{~N}$ push disturbance. Additional limits on X COM velocity result from manipulability of the COM, limiting the maximum achievable velocity. . . . . . . . . . . . . . . . . . . . 142

6.5 Phase plane trajectory of $\mathrm{Z}$ COM when pushed by a $25 \mathrm{~N}$ disturbance superimposed on the expected stable region from the LIPM model. Without the CMP controller, the originally defined COM trajectory would lie outside the stable region. . . . . . . . . . . . . . . . . . . . . . . . . . . . 143

6.6 The shaded region corresponds to the region in which the default action

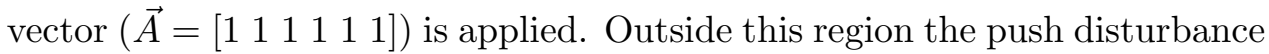

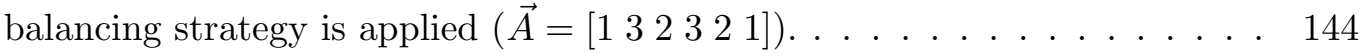


B.1 Calibration curves used to establish the parameters for the ground reaction forces estimates.$\ldots \ldots \ldots$. . . . . . . . . . . . . . . . . 169 


\title{
List of Abbreviations
}

\author{
ABL-BI Advanced Biomechatronics and Locomotion Laboratory-Biped One \\ BBC Behaviour Based Control \\ BBLC Behaviour Based Locomotion Controller \\ CAD Computer Aided Design \\ CAN Controller Area Network \\ CMP Centroidal Moment Pivot \\ COG Centre of Gravity \\ COM Centre of Mass \\ COP Centre of Pressure \\ DOF Degree of Freedom \\ DS Double Stance \\ DSL Double Stance Left \\ DSLS Double Stance Left Shifted centre of mass \\ DSRS Double Stance Right Shifted centre of mass \\ FEA Finite Element Analysis
}


GRF Ground Reaction Force

INS Inertial Navigation System

LIPM Linear Inverted Pendulum Model

ROC Rate of Change

SSL Single Stance Left

SSR Single Stance Right

ZMP Zero Moment Point 


\section{Chapter 1}

\section{Introduction}

About 4 million years ago a significant evolutionary change occurred in our ancestors - they became bipedal walkers. This allowed them to traverse greater distances more efficiently and freed their upper limbs to use tools [8]. Today, roboticists seek to build bipedal robotic walkers with many of these benefits. However, bipedal walking also has its challenges. Most importantly it is inherently less stable than most wheeled and tracked mobile bases. This is mainly a result of reducing the support polygon, the area encompassed by the feet in contact with ground. This makes it easier for the system to become unbalanced resulting in a fall and potentially causing damage. Combined with the additional challenges of complex kinematics, high computational costs and mechatronic design limits, we have yet to design and control a bipedal walking robot with the same walking abilities as a human.

Early efforts to build two legged robots were focused at Waseda University in Japan, where the first controlled dynamic robots were introduced in the early 1980s [9]. Since then, many teams have developed bipedal and humanoid robots capable of not only walking, but also, running, dancing and ascending staircases $[1,4,5,9,16]$. However, the variety of terrains on which these robots can walk remains limited primarily to flat level ground. Small irregularities in the terrain such as bumps, depressions or slopes can still easily cause even the most advanced robots to fall. The work presented in this thesis is a proposed approach to design a controller, called the Behaviour Based Locomotion Controller (BBLC) that can learn to adapt to this wide variety of disturbances. The following sections in this 
chapter present the motivations and objectives for designing the BBLC in Section 1.1 with the contributions resulting from this work summarized in Section 1.2, A chapter summary follows in Section 1.3. Finally, a general background on bipedal control methods and bipedal robots is presented in Section 1.4 .

\subsection{Motivation}

Early bipedal control systems created stable walking gaits primarily by planning the trajectory of the Centre of Mass (COM) of the robot. Initially, static stability was enforced, where the COM was constrained to lie within the support polygon. This approach limited walking speeds and as a result, the Zero Moment Point (ZMP) 17, 18 (further discussed in Section 1.4.2 was developed, which consisted of a simplified model of the robot's dynamics and allowed for quasi-dynamically-stable walking and faster walking speeds 19 21]. Researchers then began to focus on further increasing the robustness of bipedal systems to external disturbances. This led to the development of many augmented ZMP based approaches which could compensate for larger push disturbances, steps and slopes $[22[26]$. However, in attempts to further increase robustness, researchers established that COM manipulation alone was a limited approach. In recent years, this has led to the development of new balance criteria and strategies such as those based on momentum regulation $27,32,32,37]$ or modified stepping manoeuvres $22,38,43]$. However, most of these approaches are designed to compensate for specific types of disturbances known a priori. If the disturbance is unknown, and a strategy has not been developed for the disturbance, then these approaches will fail.

The primary challenge with the balance problem is its generality. The variety of disturbances is extremely large and an approach where a controller is designed for each specific disturbance quickly becomes impractical. A similar problem existed for mobile robot navigation in unknown environments. In this problem, a mobile robot was required to navigate to a position while moving through an environment without a map [44]. A 
widely successful approach to this problem was Behaviour Based Controllers (BBC) 44 (further discussed in Section 1.4.3). In these controllers, simple control behaviours such as go to goal, or move away from obstacle are combined together using a coordination method. As a result, a more complex controller emerges from this coordination, capable of solving the greater control problem of navigating in an unknown environment 44 47 . This adaptability to unknown environments was the primary motivation to apply the BBC architecture to the locomotion balance problem in the BBLC proposed in this thesis. The intent is to apply the same feature of emergent control in order to create a controller capable of adapting to the unknown environment.

\subsection{Objectives and Contributions}

The primary objectives for this work are to design, implement and validate a controller that has the following features:

- A modular behaviour-based architecture in which existing balancing behaviours can be combined together.

- The ability to learn new balancing strategies to compensate for unknown disturbances.

- A generalized architecture that can be applied to any robotic system where locomotion and balance are required.

The development of this controller resulted in the following principal contributions as presented in this thesis:

1. Robotic design of a 13-DOF bipedal robot called ABL-BI (Advanced Biomechatronics and Locomotion Laboratory Biped One) capable of performing experiments in bipedal locomotion control and balance. The design included mechanical, mechatronics, software and electrical design of the system, including the development of a unique distributed control architecture. 
2. The design of a novel robotic locomotion control architecture called the Behaviour Based Locomotion Controller (BBLC). In this architecture, Behaviour Based Control is applied to the locomotion problem by dividing locomotion into several task-space motions, for which different balancing behaviours can be planned. These behaviours are then combined together to generate new balancing strategies. A reinforcement learning algorithm is used to determine which of these balancing strategies increase robustness to different unknown disturbances. The feasibility of this architecture is initially evaluated on two different platforms: a planar biped and a mobile manipulator.

3. The implementation and validation of the BBLC on a 13-DOF biped robot in simulation. This implementation demonstrates that the BBLC is capable of generating new balancing strategies for the bipedal locomotion problem for several different unknown disturbances. Additionally, the high simulation fidelity allows for offline learning which can be transferred to the experimental platform

4. Experimental validation of the BBLC on the ABL-BI robot. The BBLC is implemented on an experimental platform, showing that the emergent balancing strategies are effective in experiment.

The contributions listed above have been recognized in the following publications:

1. Beranek, R. and Ahmadi, M. A Behaviour Based Approach to Bipedal Balance Control. IEEE Transactions on Robotics (Submitted September 2014)

2. Beranek, R. and Ahmadi, M. A Learning Behavior Based Controller for Maintaining Balance in Robotic Locomotion. Journal of Intelligent and Robotic Systems (Revised version submitted June 2014)

3. Beranek, R., Fung, H., and Ahmadi, M. Disturbance Compensation in Bipedal Locomotion Using Ground Reaction Force Feedback and the CMP. International Journal of Robotics and Automation, 2014 (Accepted and in press) 
4. Beranek, R., and Ahmadi, M. A behavior based locomotion controller with learning for disturbance compensation in bipedal robots. IEEE International Conference on Robotics and Automation, 2012 (pp. 1915-1920).

5. Beranek, R., Fung, H., and Ahmadi, M. A walking stability controller with disturbance rejection based on CMP criterion and ground reaction force feedback. IEEE/RSJ International Conference on Intelligent Robots and Systems, 2011 (pp. 2261-2266).

6. Barker, O., Beranek, R., and Ahmadi, M. Design of a 13 degree-of-freedom biped robot with a CAN-based distributed digital control system. IEEE/ASME International Conference on Advanced Intelligent Mechatronics, 2010 (pp. 836-841).

\subsection{Thesis Overview}

\section{Chapter 2: Design of ABL-BI and Simulation Tools}

In this chapter, the design of ABL-BI is presented. This includes an overview of the conceptual design, actuator selection and detailed design. Additionally, the simulation tools that were developed for controller testing are presented. These include a simplified planar bipedal simulation and a 3D dynamic simulation of ABL-BI.

\section{Chapter 3: Behaviour Based Locomotion Controller - Architecture and Imple- mentation Examples}

In this chapter, the general architecture of the BBLC is presented. This includes a formal mathematical formulation of the controller and presentation of the generalized architecture, as it could be implemented on any platform. This is followed by two examples of a BBLC implementation: (1) a planar biped and (2) a mobile manipulator. For both these examples, the details of how the BBLC is implemented are presented along with simulation results. The objective of presenting these two examples is to show the generalizability of the BBLC to different robotic systems and demonstrate the feasibility of the BBLC. More detailed analysis of the BBLC is presented in Chapters 4 to 6 . 


\section{Chapter 4: BBLC Implementation on ABL-BI: Simulation}

This chapter presents the full implementation of the BBLC on ABL-BI as well as simulation results. This includes three different disturbance cases (push, slope and step) in addition to walking on flat level ground. For each case the BBLC learns a new balancing strategy which can compensate for these disturbances with no a priori knowledge of the disturbance.

\section{Chapter 5: Experiments with ABL-BI}

Experimental results using the ABL-BI robot are presented. These experiments consist of reproducing the test cases examined in simulation in Chapter 4. The primary objective in this chapter is to examine if the learnt balancing strategies from the simulation are also activated on the experimental platform and if they increase the robustness to disturbances. Overall, the experiments are used to validate the results achieved in simulation.

\section{Chapter 6: Stability Analysis of Behaviour Strategies Using the Linear Inverted Pendulum Model}

An analytical evaluation is performed on some of the balancing strategies that were generated as part of the results presented in Chapter 4. This analysis is completed to gain a better understanding of how these strategies augment the balance of the biped and why the BBLC selects them. This is accomplished by performing a stability analysis of the linear inverted pendulum model and establishing how different behaviours affect the stable region defined in the model.

\section{Chapter 7: Conclusions and Recommendations}

This chapter summarizes the work presented in the thesis, the conclusions that can be drawn from the experimental results and their implication to balance control. 

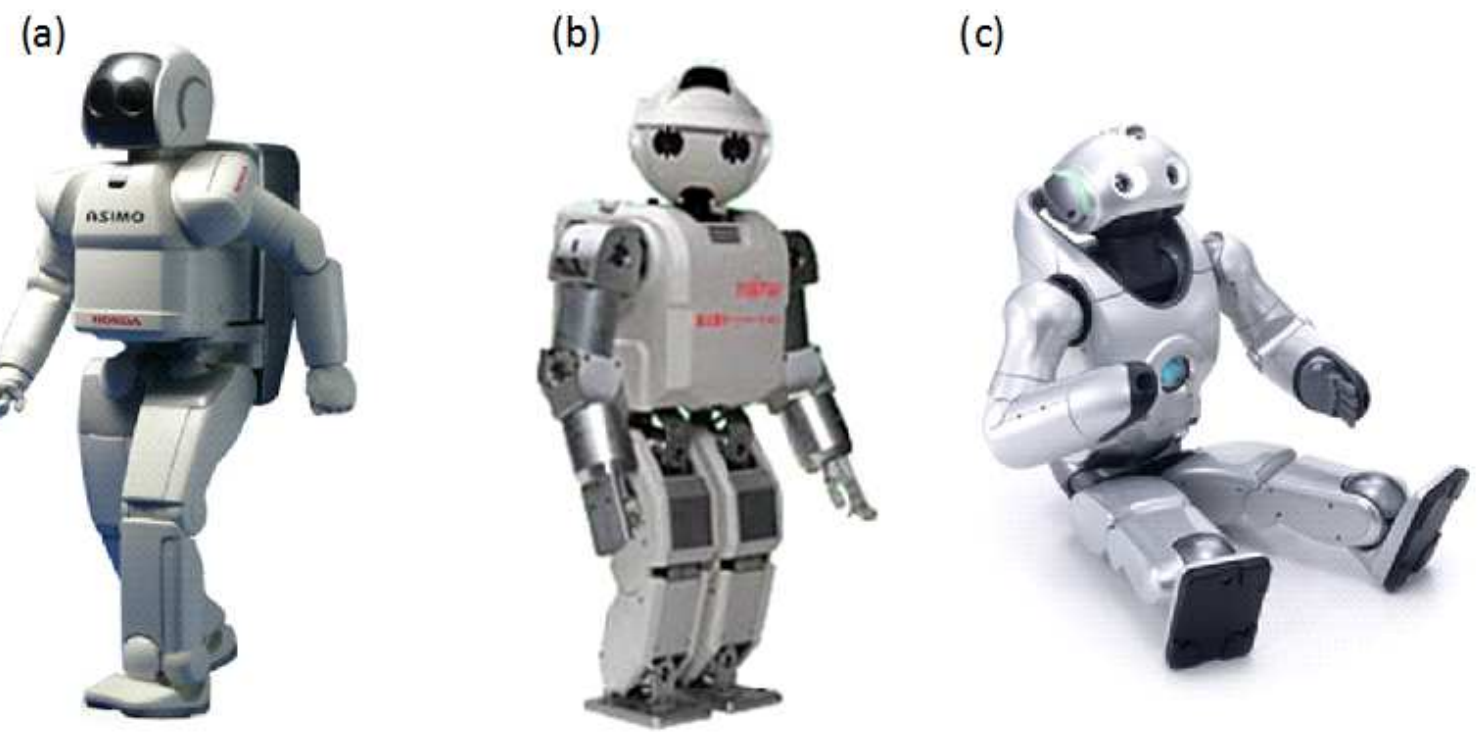

Figure 1.1: Humanoid robots designed in industry (a)ASIMO by Honda corporation 1] (b) HOAP-3 by Fujitsu [2, (c) QRIO by Sony [3].

\subsection{Background}

\subsubsection{Biped and Humanoid Robots}

The complex dynamics of bipedal robots, including the difficulty to accurately model ground contact, make it difficult to develop high-fidelity simulations. As a result, experimental verification on a biped or humanoid robot is always required to truly validate a controller design. Designing a biped or humanoid robot has many challenges ranging across several fields of engineering, including mechanical design, software design, communications architecture and controls engineering. As a consequence, many different research groups from academia and industry have designed bipedal and humanoid robot prototypes to resolve these design challenges. The most famous humanoid is ASIMO from Honda [1, 9, shown in Figure 1.1 [1]. Other robots have been designed in industry by Toyota, Fujitstu [2] and Sony 3]. The recent Darpa Robotics Challenge has led to the development of several new humanoid robots from both academic teams as well as private companies such as Boston Dynamics and Schaft (acquired by Google) [48]. 
(a)

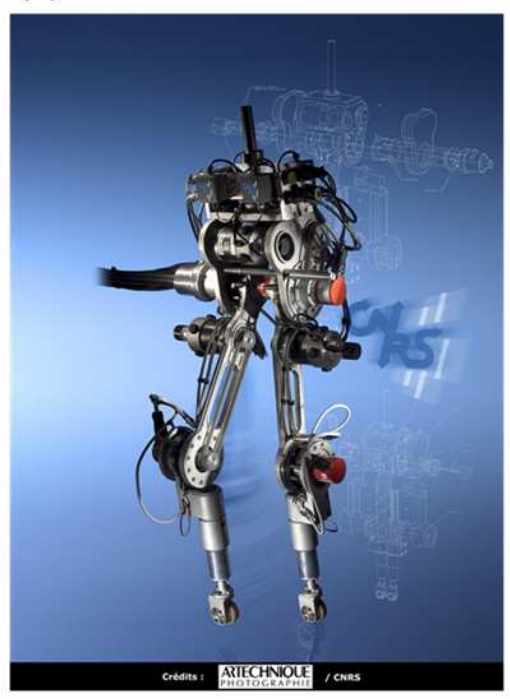

(b)

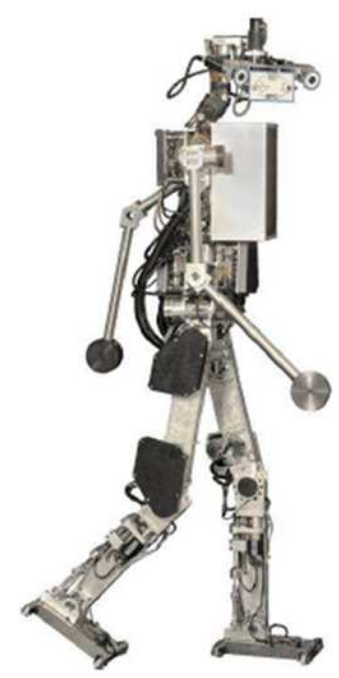

Figure 1.2: Research biped platforms: (a) Rabbit [4] (b) JOHNNIE [5].

Given the costs associated with these robots designed by industry, many biped and humanoid robots have been designed in academia, ranging from very simple, planar bipedal robots to complete humanoid robots [3, $5,13,15,49,57]$, some of which are shown in Figure $1.2[4,5]$. These robots are prototypes and are not available commercially. A final class of humanoid robots is designed for entertainment purposes. These robots are usually servo actuated, with large feet and are capable of performing various motions, but they do not provide the required low-level access to their controllers to perform research [3]. These robots present a wide range of different designs and capabilities, both for research and other purposes. However, there are very few platforms commercially available for research purposes. This was the primary motivation to design and build a robot for the purposes of the research presented in this thesis. 


\subsubsection{Biped Locomotion Controllers}

There are several factors that have made the problem of stabilizing bipedal robot locomotion a challenging one. The fundamental challenge is that the system is under-actuated given that the contact between the robot's feet and the ground is not rigid. This means that the forces required to stabilize the robot cannot be directly applied. Stabilization forces can only be generated indirectly through the robot's motion. Additionally, the dynamics of the system are highly nonlinear given the large number of DOFs and discrete nature of walking (intermittent foot contacts). The large number of actuated DOFs also introduces redundancy in the system, i.e., the number of DOFs exceeds the number of joints required to perform a task-space objective. Finally, these control-based challenges are also compounded by the mechatronic design challenges typically associated with designing biped robots.

Given the complexity of the problem, there have been many different approaches to generating stable walking gaits in bipedal robots. The most successful algorithms are capable of creating stable walking and running gaits on flat terrain in real-time [1,16]. These controllers have been developped in a combination of simulation and experimental work. Section 1.4 .2 gives an overview of the most common approaches to this control challenge. However, in each of these approaches there are usually the following common elements:

- Stability criterion: The concept of stability in bipedal robotic systems remains ill defined [17]. Therefore a variety of stability criteria exist, usually based on a simplified model of the robot's dynamics and/or studies into human posture stability [17].

- Model: The exact dynamics of a multi-DOF bipedal or humanoid robot, including a ground contact model, are highly nonlinear and are therefore challenging to model, analyze or use for controller development. A simplified model of the robot is usually used for controller development $6,20,21,23,58$.

- Redundancy resolution: Bipedal and humanoid robots have a high number of 
redundant actuated DOFs. Almost any desired motion of the robot has infinite solutions. Every controller has some means of resolving this additional redundancy, and this will impact the robot's stability 21,59 .

\section{ZMP Criterion Based Controllers}

The Zero Moment Point (ZMP) criterion stems from research into biomechanical properties

of walking as far back as the $19^{\text {th }}$ century [17]. However, it was initially proposed as a criterion for bipedal locomotion stability by Vukobratovic [17]. The ZMP is the point where the ground reaction force (GRF) acting on the system must act to negate all the moments acting on the robot. If the ZMP remains in the support polygon, formed by the feet in contact with the ground, balance will be maintained and the robot will not tip over [17]. If the ZMP is not within the support polygon, then the moments cannot be compensated by the GRFs; the robot's foot will roll on its edge and the robot will tip over.

The ZMP stability criterion gave a definition of stability (balance can be equally used instead of stability in this case) in bipedal locomotion that could be effectively used in control algorithms to generate stable gaits. However, two key challenges remained. Determining the ZMP from the robot's dynamics was challenging theoretically and computationally, given the complex nonlinear dynamics of the robot. Also, there was no clear way of manipulating the ZMP position to maintain stability [17].

Both these issues were resolved by introducing the linear inverted pendulum model (LIPM) 6, $19,21,23$. As shown in Figure 1.3 [6], this model consisted of simplifying the legged system's dynamics to a simple cart and pendulum model, where the lumped mass of the pendulum represents the centre of mass $(\mathrm{COM})$ of the entire body. Using this model the dynamics of the system can be simplified as 6

$$
\ddot{x}_{C O M}=w_{G}^{2}\left(x_{C O M}-x_{Z M P}\right),
$$




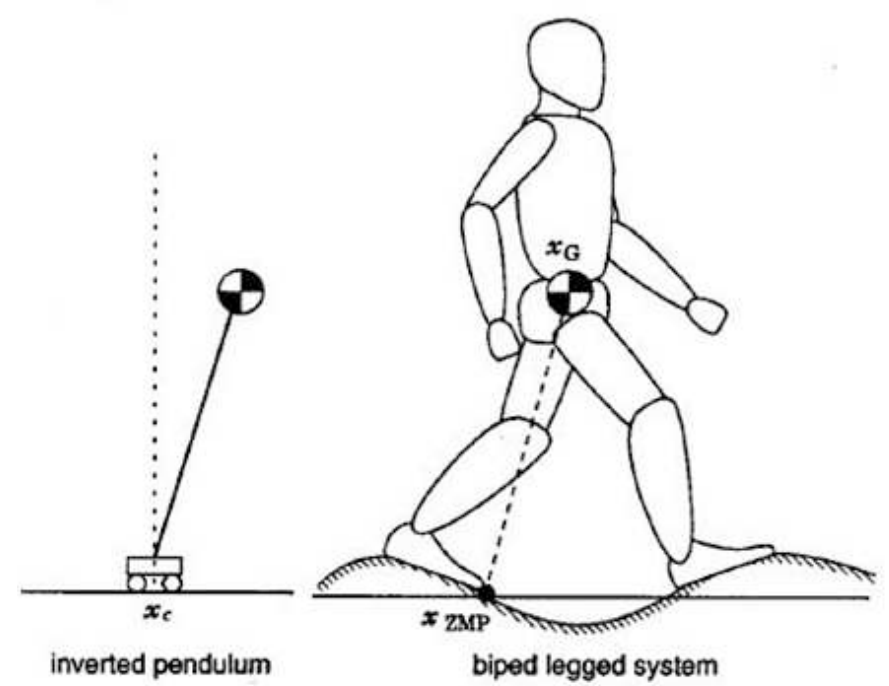

Figure 1.3: Linear inverted pendulum model for humanoid robots [6].

$$
\ddot{y}_{C O M}=w_{G}^{2}\left(y_{C O M}-y_{Z M P}\right),
$$

where

$$
w_{G}=\sqrt{\frac{\ddot{z}_{C O M}+g}{z_{C O M}-z_{Z M P}}},
$$

and $x_{C O M}, y_{C O M}, z_{C O M}$ are the positions of the COM, $x_{Z M P}, y_{Z M P}, z_{Z M P}$ are the positions of the ZMP and $g$ is the acceleration due to gravity. This model was validated with studies in human walking [34], and the LIPM was found to accurately approximate the position of the ZMP during stable walking [18, 33, 35].

From these dynamic equations, there exists a clear relationship between the COM of the legged system and the ZMP. This relationship was exploited to design reference COM trajectories that would maintain the ZMP within the support polygon of the robot. The second step in ZMP controllers is to plan the motion of the robot such that the COM matches the reference COM. Again, the complex kinematics of the robot make this a challenging task. Early ZMP-based controllers placed large masses in the robot's torso 60, 61]. This allowed for the COM of the robot to be effectively controlled by pitching or rolling the torso 
by small angles. Later, a more complete algorithm was designed that uses all of the robot's joints to move the COM. The whole body cooperative method (WBC) 66, 21, 59, 62, uses the velocity level kinematics relating the joint angular velocities, $\overrightarrow{\dot{\theta}}$, to the COM velocity, $\overrightarrow{\dot{X}}_{C O M}$, as

$$
\overrightarrow{\dot{X}}_{C O M}=J_{C O M} \overrightarrow{\dot{\theta}}
$$

where the Jacobian, $J_{C O M}$, is defined as

$$
J_{C O M} \equiv \frac{\partial \vec{X}_{C O M}}{\partial \vec{\theta}}
$$

Given a reference COM velocity $\overrightarrow{\dot{X}}_{r C O M}$, the inverse relationship from 1.5 can be used to find a set of reference joint velocities $\overrightarrow{\dot{\theta}}_{r}$ as

$$
\overrightarrow{\dot{\theta}}_{r}=J_{C O M}^{\dagger} \overrightarrow{\dot{X}}_{r C O M}
$$

The reference joint velocities can then be integrated to get a set of reference joint positions that will move the COM to the desired position. Note that the pseudoinverse, $J_{C O M}^{\dagger}$, is used to find the inverse Jacobian. This is due to the high number of actuated degrees of freedom in the robot, causing the Jacobian matrix to be non-square.

The WBC approach allowed for stable walking gaits to be generated for many different robots $[1,5,9,16,20,54,62,66$, initially using offline pre-planning of the motion, to more recent implementations, that are capable of generating stable gaits in real-time [6, 20]. However, several limitations have emerged, mainly stemming from the use of the LIPM model which makes the following assumptions:

- Flat foot contacts are made with the ground during single stance and double stance.

- No foot slipping occurs.

- The robot walks on flat smooth ground.

- The LIPM model accurately predicts the ZMP position.

- The only external forces acting on the system are the ground reaction forces. 
If the terrain becomes uneven or the motion of the robot becomes too quick then this approach can no longer be used given that the LIPM no longer provides an accurate mapping between the COM position and the ZMP [17]. To address some of these issues, several variations of the LIPM have been developed [29, 32, 58, 64, 67]. These new models mostly consist of increasing the complexity of the mass-cart model by adding additional masses, bodies with inertias and/or spring-damper connections between the cart and the pendulum mass. These models have allowed for faster walking and walking on sloped terrains, but still have not allowed for walking on non-flat terrain or compensating for external disturbances.

Another issue that has received little study in the literature is the coordination of the COM position control with other task-space control objectives required to generate a stable gait. These task-space objectives consist of [21,34]:

- Moving the swing leg.

- Maintaining flat foot contact.

- Maintaining upright torso posture.

- Moving the COM.

- Modifying the control algorithm to generate compliance in joints to minimize impact forces.

Additionally there are constraints to the robots motion that must be considered:

- Avoiding singular positions for both COM position control and swing leg position control.

- Prevent self collisions.

- Prevent foot slip during double stance. 
There are several approaches that have been used in current ZMP controllers to address these different objectives and constraints. Swing leg motion control is generally controlled in parallel with the COM position control 62,68. Some joints are dedicated to controlling the swing leg position while others are used to control the COM position. Other task-space objectives are satisfied by careful preplanning of the reference COM motion and/or by applying additional constraints to the COM position control. These approaches generally result in a controller that can successfully generate a stable periodic gait. However if there are significant variations from pre-panned trajectories, or if the robot is disturbed, these approaches generally fail 62,63. Also, these coordination methods can lead to some controllers hindering others; e.g., moving the swing leg independently can interfere with COM control. Overall, there is no unified approach to coordinate the control such that the constraints and task-space objectives listed above are satisfied.

In summary, the ZMP stability criterion, coupled with the LIPM have provided a framework that has allowed for successful generation of stable periodic walking gaits for bipedal robots on flat terrain. However, robustness to unknown external disturbances or aperiodic changes to the gait have not been achieved using the ZMP framework.

\section{Virtual Model Control}

In ZMP-based methods the stable gaits are generated by planning a COM trajectory that will maintain the ZMP within the support polygon based on the LIPM model of the biped. Virtual Model Control (VMC) moves away from the COM manipulation paradigm to a force based control strategy. The concept of VMC is that the robot can be stabilized intuitively by applying external forces to the robot as shown in Figure 1.4 (b) 7,69 . These virtual

external forces can be effectively reproduced by applying the correct joint torques at each individual joint which can be computed by the Jacobian transpose relationship as

$$
\vec{\tau}=J_{A B}^{T} \vec{F},
$$


(a)

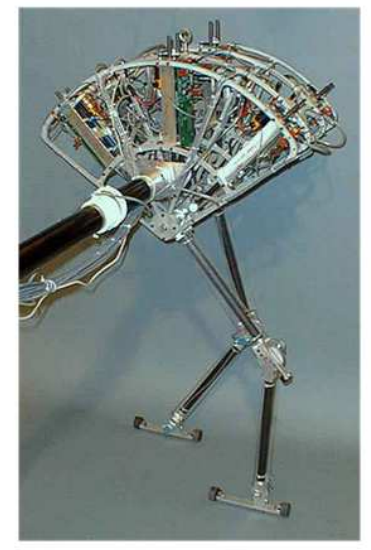

(b)

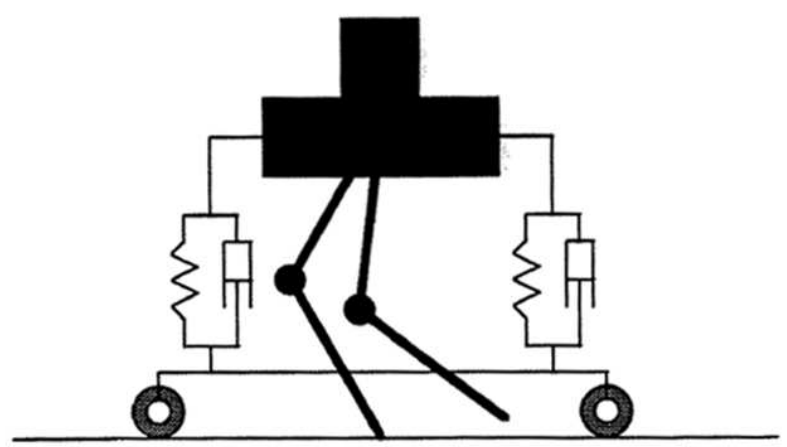

Figure 1.4: (a) Spring flamingo robot with series elastic actuators [7], (b) virtual spring damper forces are applied to the biped to stabilize it [7].

where $\vec{\tau}$ is the vector of all joint torques, the Jacobian $J_{A B}$ is the kinematic Jacobian between any base frame $A$ and frame $B$ on the robot and $\vec{F}$ is the vector of virtual forces that is applied at frame $B$. The virtual forces are then modelled as spring-damper connections that act to stabilize the robot's motion as shown in Figure 1.4 (b) [7]. Unlike in the ZMP based approach, there is no explicit definition of stability. Instead, virtual forces are designed to maintain the robot's height, velocity and torso orientation within predefined values. The controller attempts to maintain these parameters near values that have been experimentally found to correspond to a balanced posture and stable forward walking speed. Additionally, the task-space objectives such as swing leg control can be accomplished by applying a separate set of virtual forces at the tip of the swing leg [7].

This method was extensively tested in planar simulations where the controller demonstrated the ability to generate stable walking on flat terrain and was able to adjust to terrains of varying slopes by modifying the virtual force model [7. Given that a dynamic model of the robot is not used for this approach, the virtual forces must be tuned experimentally in order to stabilize the robot's gait. The key limitation with this method is that for the virtual forces to be reliably reproduced by the relationship in (1.7), the 
reference joint torques must be accurately reproduced by the joint actuators. In traditional electrically actuated bipedal robots this is difficult to accomplish [7]. A robot with series elastic actuators or joint torque sensors is required so that accurate feedback of the joint torques being applied can be used in the control system. Thus far, Spring Flamingo, shown in Figure 1.4 (a), is the only biped robot with the appropriate actuators where VMC has been successfully tested.

\section{Reinforcement Learning}

Due to the complexity of the bipedal control problem, its nonlinear intermittent dynamics, under actuated nature, and redundant DOFs, analytical derivation of a control algorithm is beyond the capability of existing control design techniques. The controllers presented in the previous sections used two different approaches to solve the bipedal control challenge. The ZMP based approach used a simplified model of the system in order to derive a control law while VMC used an experimental approach to determine a virtual force model that can stabilize the robot. More recently a new approach has emerged, where machine learning algorithms are applied to learn the control algorithm rather than designing it a priori 70 98. More specifically, the biped control problem has been formulated as a reinforcement learning problem. In this framework, as illustrated in Figure 1.5, a control law is learnt by the system by trying different control actions and evaluating the resulting state. Actions resulting in desirable states, based on the control objective, are kept in memory to be used again in the future. There are three key components to reinforcement learning: (1) The actions applied to the system by the controller, also known as the policy or controller output; (2) the reward function, defined by the designer, evaluates whether the current state of the system is desirable or not based on the control objective; (3) the value function estimates the total accumulated reward (present reward plus sum of future reward from the reward function) if a given action is performed at the current state of the robot. In other words, the value function provides a measure of how much reward a particular action will generate over time if it is applied. From the value function, the 


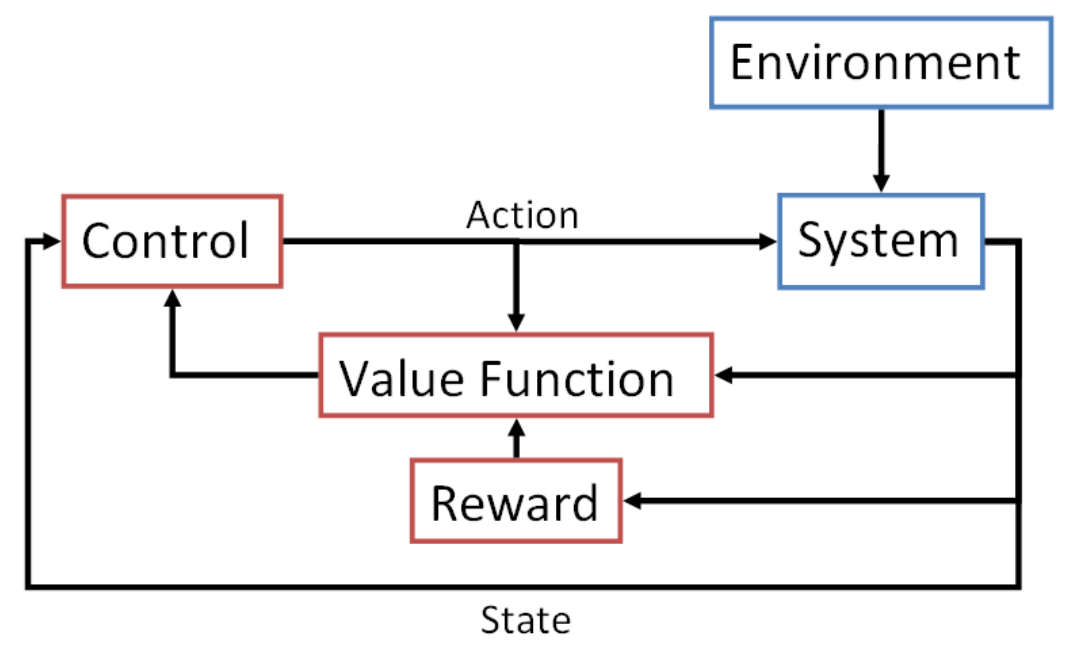

Figure 1.5: Reinforcement learning block diagram.

action that is applied to the system is selected by finding the action that will maximize the value function at a particular state; i.e., the action that is expected to provide the most accumulated reward is selected. The value function is generally not known; therefore, the objective of the reinforcement learning problem is to approximate the value function. Different function approximation tools such as lookup tables, neural networks and support vector machines [99] are used. The learning task then consists of iterating through different state-action pairs to determine which actions generate the most reward. The reward information is used to update the approximation of the value function. If the system is simple, all state-action pairs can be visited and the exact value function can be found. If the system is more complex, it may be impractical to test all state-action pairs. Hence, an additional algorithm will be required to determine which state-action pairs should be visited. A typical example is a stochastic search algorithm. In this approach different actions are tested randomly until the approximation of the value function converges.

\section{Learning Controllers}

In the biped control problem, the reinforcement learning architecture has been used to learn control policies that will successfully generate stable walking gaits. In all the biped 
learning approaches a common reward function is used. If the robot stays upright and moves forward, a positive reward is given and if the robot falls, a strong negative reward is given. However, several different approaches have been used to learn policies that will generate stable walking $70,87,89]$. Initially a direct approach was used, where the policy consisted of generating reference joint angles depending on the robot's state $70,95,96$. This approach consists of a problem with a high number of continuous states and policies, resulting in very long learning times that were impractical except for simple planar bipeds with five joints or less. As a result, the control policies have been simplified by two main approaches: (1) Simplify the walking gait to parameters such as stride angle and phase $71,73,74,91$ and (2) use coupled Central Pattern Generator nonlinear oscillators as a base trajectory for the gait $76,87,88,90]$.

In the first approach, a base walking trajectory is generated using a control approach such as ZMP. The reinforcement learning algorithm is then used to modify some parameters in the walking gait. This approach reduces the dimensionality of the action space of the algorithm, from every actuated DOF to a smaller set of walking parameters which can be tuned. This reduces the size of the learning problem by reducing the number of state-action pairs, allowing for approximation of the value function to converge with less iterations. In their approach, Morimoto et al. 91 modified the maximum hip angle as well as the phase between the right leg swing and left swing to modify a walking gait to adapt to sloped terrains. In general, this technique has been successful in adapting stable gaits on flat terrain to periodic walking on sloped terrain as well as increased walking speed or energy efficiency.

The second approach is to use nonlinear oscillators to generate stable gaits. Given that the walking motion is periodic, nonlinear oscillators generate a signal that can be used to provide a base trajectory. Similarly to the previous approach, the neural oscillators provide a base gait that can be used in the controller. In their work, Endo et al. 76, 87, 88, used neural oscillators to generate the nonlinear periodic motion for each joint in a humanoid 
robot. Additionally, several joint motions were coupled with each other to reduce the dimensionality of the learning problem to a small number of entrainment variables, which varied the phase and amplitude of the neural oscillator output. The learning algorithm consisted of varying the entrainment variables until a stable gait was found. This method was successful in generating stable walking gaits as well as walking gaits capable of turning, in both simulation and experimental tests [76].

The key advantage of learning based approaches is that explicit modelling of the system and environment is not required. Rather, feedback comes in the form of a reward function which evaluates the controller's performance $76,87,88,90,100]$. Another advantage is that the gait is not limited by any definition of stability such as ZMP. This allows the algorithm to explore gaits which may violate a stability criterion in an attempt to improve efficiency or walking speed. The key limitation of learning approaches is that current methods only allow to find gaits which work on level or sloped terrain, without disturbances applied to the robot. As the environment becomes more varied, such as changes in terrain as well as adding disturbances, the motion of the robot can no longer be periodic. Hence, the assumptions used to simplify the problem in current learning approaches can no longer be applied. The learning task increases in dimensionality and becomes impractical to solve.

\section{Momentum Based Controllers}

Recent studies in human walking biomechanics have indicated that total angular momentum is a highly regulated parameter during walking [32, 33, 35]. Based on this observation, some researchers have attempted to include regulation of angular momentum in bipedal stability controllers $18,27,29,32,33,41,42,68,101,102$. The first approach was proposed by Kajita [27] in his resolved momentum control approach. In this controller, the ZMP based control approach is augmented to regulate total angular momentum about the COM by modifying the reference COM motion via inverse dynamics. This method was success-

fully tested on HRP-2 (a humanoid robot designed by Kawada Industries Inc.) [51 and was 


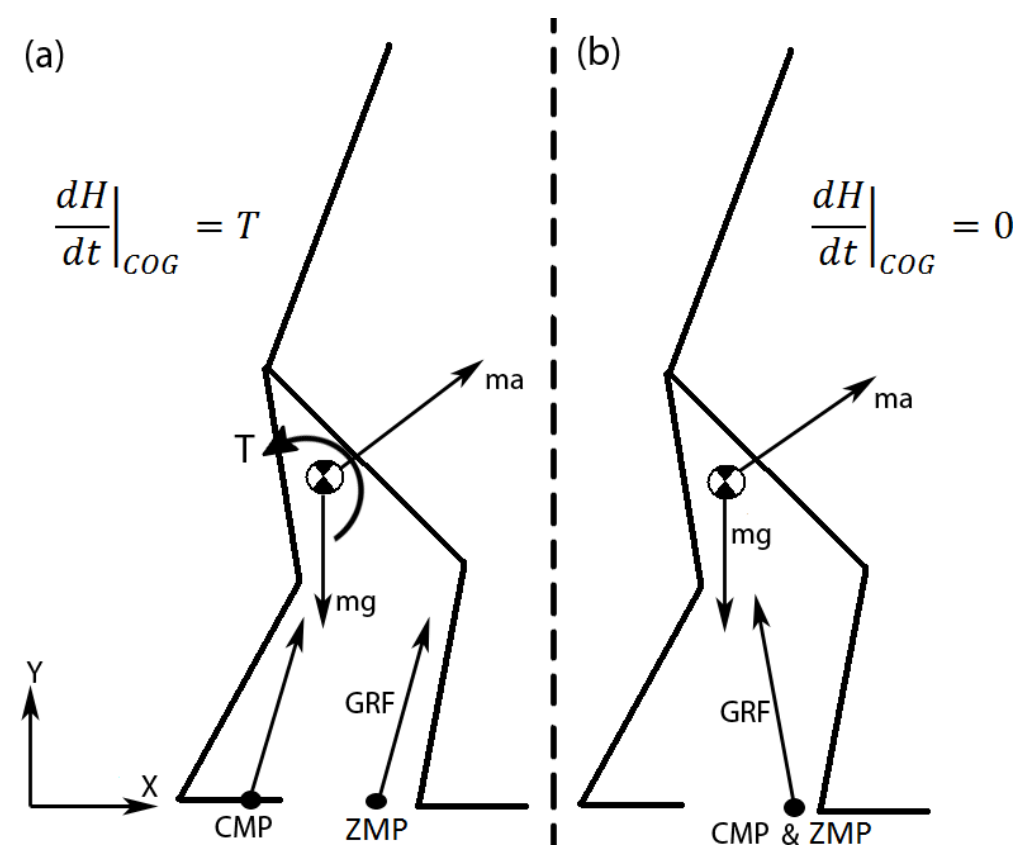

Figure 1.6: The location of the ground reference points CMP, ZMP, and COM when there is (a) an uncompensated moment acting about the COM, and (b) zero moment acting about the COM.

shown to generate stable walking.

Recent research has proposed a new stability criterion, known as the centroidal moment pivot (CMP), based on regulating spin angular momentum (rate of change of angular momentum) about the COM 18. As shown in Figure 1.6 (a), the CMP corresponds to the point on the ground where the ground reaction force vector must act to intersect with the position of the COM . If this criteria is satisfied, the rate of change of angular momentum $\frac{d H}{d t}$ will be zero 18 . If there is an uncompensated moment, $T$, acting on the robot, which may be caused by a disturbance, the CMP will diverge from the ZMP as in Figure 1.6 (a). Otherwise, if the robot is not disturbed the CMP and ZMP will be coincident, and there will be no uncompensated moment acting about the COM, as shown in Figure 1.6 (b). Given the sensitivity of the CMP to disturbances acting on the robot, it has been suggested that this stability criterion can be used in a controller to compensate for disturbances acting on the robot [18,32. Thus far, two implementations 


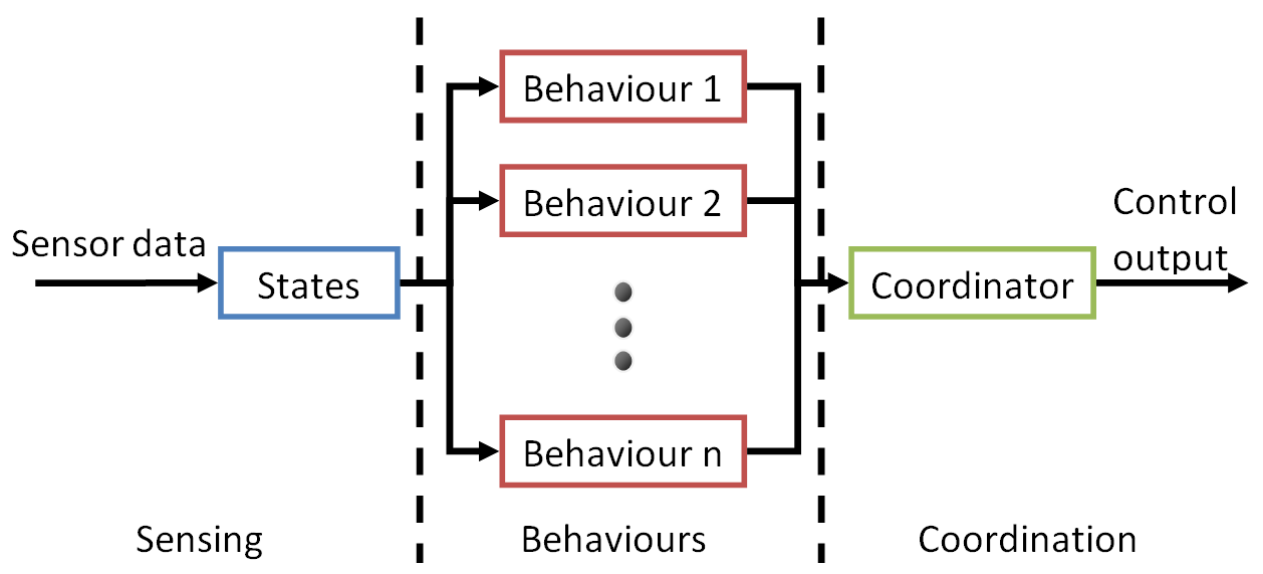

Figure 1.7: Behaviour based control architecture.

of the CMP criteria have shown in planar simulations that using the CMP as a stability criterion may be used to increase disturbance compensation of the controller $37,68,103$.

\subsubsection{Behaviour Based Control}

The founding concept of behaviour-based control is the concept of emergence, where complexity arises from a simple set of rules without being explicitly designed into the system [44]. In the general behaviour-based scheme, shown in Figure 1.7, the system is divided into three separate modules: sensing, behaviours and coordination 44, 45. In the sensing module, sensors are used to measure states that may be relevant to the control tasks. In the second module, individual behaviours use the sensor data to compute a desired control output to be applied on the system. Each behaviour corresponds to a different control objective and is computed in parallel with the others. The last module, the coordinator, coordinates the different control outputs from each behaviour. It is through the coordination that a more complex control emerges, without being defined a priori.

There are two main classes of coordination algorithms, shown in Figure 1.8, The first is the competitive algorithm, where the coordinator selects the control output from only one behaviour to be applied to the system. This coordination method is also known as 

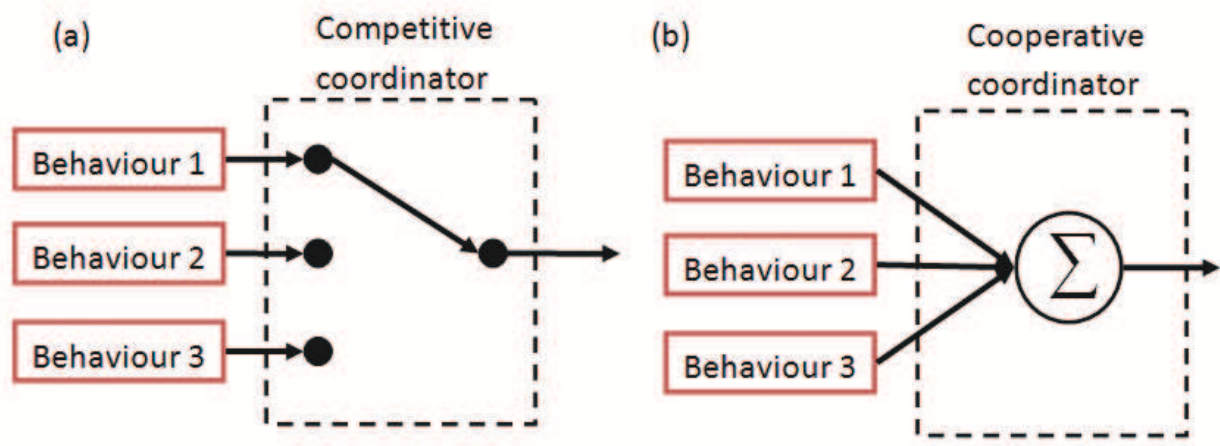

Figure 1.8: Coordination architectures for behaviour-based systems; (a) competitive, (b) cooperative.

arbitration. The algorithm determines which behaviour has the highest priority given the current state of the system [44]. The second approach is a cooperative method, where the control outputs of all the behaviours are fused together to compute the final control output to be applied on the system [44. There are various frameworks in which this approach is used, for example the potential field method for mobile robot motion planning [44, 104]. The coordination algorithm can also have a hybrid architecture, where competitive and cooperative approaches are combined. In this approach, some behaviours are arbitrated while others are fused together to generate the final control action 105.

A typical example where $\mathrm{BBC}$ is used is in mobile robotics. In this application, the behaviours are typically "go to goal" and "avoid obstacles" 44, 106, 107]. Each behaviour will specify a different velocity vector for the robot to follow. Both of these outputs are then coordinated to define a final velocity vector that satisfies both objectives $45,104,106,109]$.

Controllers using $\mathrm{BBC}$ have several advantages. These controllers do not require a model of the environment or a model of the forces acting on the system. It also simplifies controller design, where each behaviour is designed as a controller that individually satisfies a single control objective. This provides a modular architecture where different behaviours can be added or modified without affecting other aspects of the control [44,105]. Overall, 
this bottom-up design architecture has solved many control and artificial intelligence problems that could not be solved by traditional control design techniques, where explicit modelling of the system and environment were required [44].

The $\mathrm{BBC}$ architecture is adapted in this work for the robotic balance problem where it is used to combine several balancing behaviours together to generate emergent balancing strategies. Some previous works of adapted BBC previously for more complex systems, including bipedal robots $110-112$. These works focused on the motion coordination and activation of certain balancing behaviours in complex redundant systems. The BBLC presented in this work also augments BBC for redundant architectures in a unique three layered architecture. Additionally, unlike in these previous works, the focus of the BBLC is on generating emergent balancing strategies to unknown disturbances, including a learning algorithm. 


\section{Chapter 2}

\section{Design of ABL-BI and Simulation Tools}

This chapter introduces the design of ABL-BI (Advanced Biomechatronics and Locomotion Laboratory Biped One), a 13 DOF bipedal robot that was designed to evaluate controllers designed as part of the research. The development of this robot required a complete robotic system design, including mechanical design, mechatronic design, software development and electrical system design. Additionally, this chapter presents 2D and 3D biped dynamic simulations that were developed for controller development. The 3D dynamic simulation is also used as a platform to perform offline machine learning which is then transferred to the experimental platform.

Section 2.1 presents the design process that was used to develop ABL-BI, design requirements and basic kinematic equations that are used throughout the thesis. Section 2.2 introduces two simulation tools that were developed to facilitate this research: (1) a planar biped simulation and (2) a full 3D dynamic simulation of ABL-BI.

\subsection{ABL-BI Design}

The original objective for designing ABL-BI was to build a robotic bipedal platform to perform research in two legged locomotion, balance and walking energetics. At the time of the design, there were no bipedal robots with an open control architecture that were 
commercially available to perform such research. As such, a custom designed robot was developed. The following sections present the design process and completed design of the ABL-BI robot.

\subsubsection{Initial Requirements}

The general objective which dictated the design requirements was to have an autonomous robot capable of 3D walking, approximately the size of a 10 year old child and with anthropomorphic dimensions. This requirement was generally set to keep the robot mass weight to be manageable for one operator, as well as keep part costs manageable. From this objective a set of requirements was determined and listed in Table 2.1.

\subsubsection{Mechatronic Design}

The first step in the design process was concept generation, which primarily focused on different actuation concepts. Many different actuation technologies were considered such as hydraulic actuators, pneumatic actuators, pneumatic muscles and DC motors. It was decided early in the design process that an electric actuation system would be the easiest to control and most appropriate for the type of research that ABL-BI would be used for. This resulted in several concepts being developed, some of which are shown in Figure 2.1. in which different placements of actuators and transmissions were considered.

The following step was to evaluate these concepts. The primary evaluation criteria was the required joint torques for each design, given that higher required torques result in the need for larger, heavier actuators. To size the actuators correctly, a dynamic model was developed using the dimensions in Table 2.1, and using Webots ${ }^{\mathrm{TM}}$ modelling software. This dynamic model allowed for an evaluation of the torques required in the system while varying other design parameters such as the mass of links and the placement of joints. Webots $^{\mathrm{TM}}$ also generates a visualization of the model as shown in Figure 2.2. Given 
Table 2.1: Design requirements for the ABL-BI robot.

\begin{tabular}{l|l}
\hline Dimensions & \\
\hline Height & $1.0 \mathrm{~m}$ \\
Width & $0.35 \mathrm{~m}$ \\
Depth & $0.25 \mathrm{~m}$ \\
Leg length & $0.6 \mathrm{~m}$ \\
Upper leg length & $0.3 \mathrm{~m}$ \\
Lower leg length & $0.3 \mathrm{~m}$ \\
Torso height & $0.4 \mathrm{~m}$ \\
\hline Kinematics & \\
\hline Hip roll & $\pm 45^{\circ}$ \\
Hip pitch & $-45^{\circ}$ to $40^{\circ}$ \\
Hip yaw & $\pm 45^{\circ}$ \\
Torso roll & $\pm 35^{\circ}$ \\
\hline Weights and Payload & \\
\hline Total mass & 15 to $25 \mathrm{~kg}$ \\
Support for additional sensors & \\
Ability to upgrade to a humanoid (add arms and head) \\
Ability to support assistive walking devices & \\
\hline Actuation & \\
\hline Low weight & \\
High power to torque ratio & \\
Low friction & \\
Backlash below 10 arcmin & \\
Low friction in transmission & \\
\hline
\end{tabular}




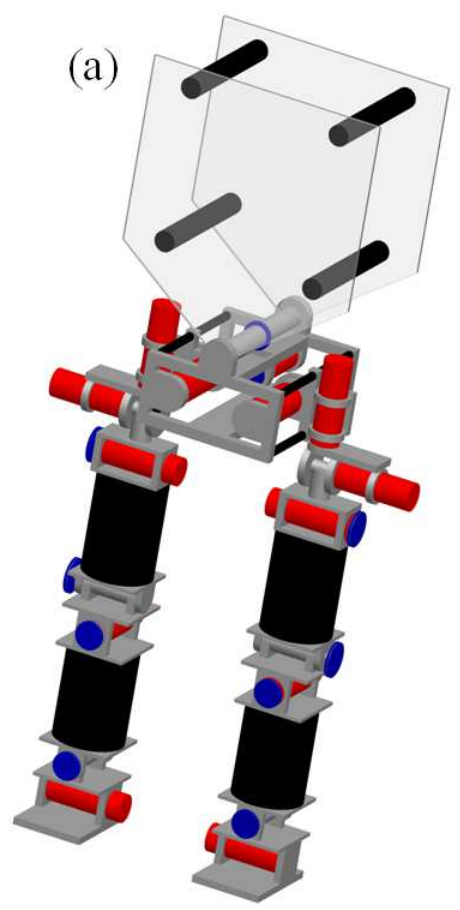

(b)

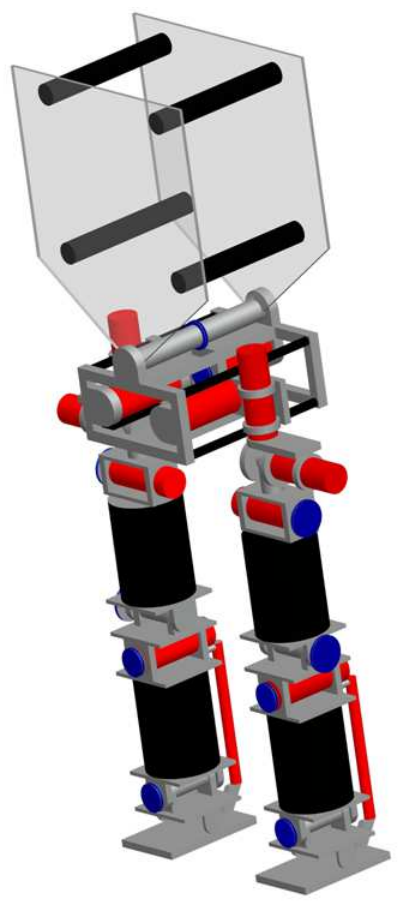

(c)

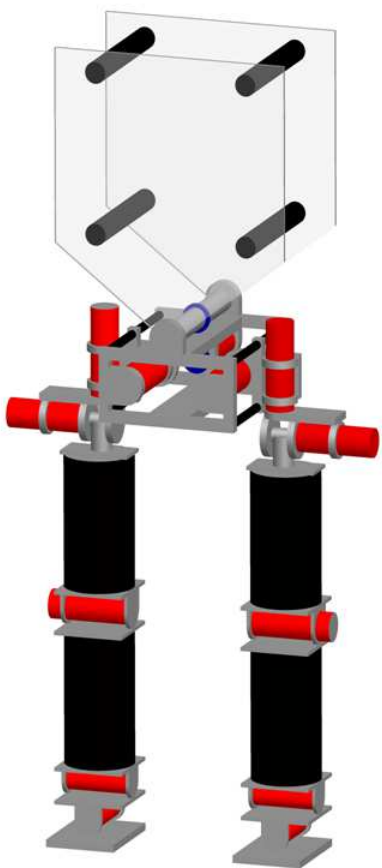

Figure 2.1: Different conceptual designs initially developed for ABL-BI: (a) DC gearmotor actuation concept with pulley transmission, (b) DC gearmotor actuation concept using pulley transmissions and a linear actuator for the hip pitch joint and (c) using direct actuation of each joint using DC gearmotors.

that a walking controller had not been developed yet at this point in the design, required actuator torques were determined by moving the biped to stances at the limit of the joint ranges and to the limit of statically balanced postures. A sample of stances which were considered are shown in Figure 2.3. An example of a resulting joint torque curve is shown in Figure 2.4. This figure shows the torque applied to the right hip roll joint for the stance test shown in Figure 2.3 (a). For this test, the maximum torque for the right hip roll joint peaks at $45 \mathrm{Nm}$. This analysis was extended for all joints and possible stances to establish average and peak torque requirements for each joint, as listed in Table 2.2. The concept that was chosen consisted of directly actuating each joint with DC gearmotors. This concept represented the option with the simplest mechanical design and lowest torque requirements. The actuators sized to meet the established torque requirements in the most lightweight package. The selected actuators and gearboxes are listed in Table 2.3 . 


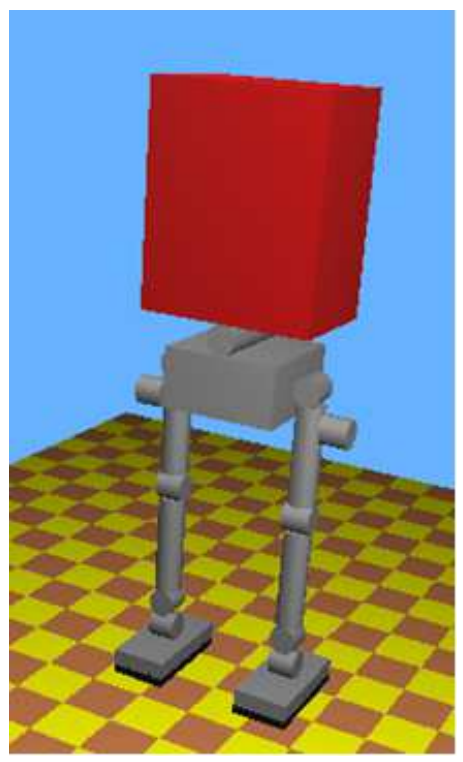

Figure 2.2: Graphical representation of the initial dynamic models used for actuator sizing and concept evaluation using Webots ${ }^{\mathrm{TM}}$ software.

Table 2.2: Estimated peak and average torques for each joint.

\begin{tabular}{r|l|l} 
Joint & Maximum Torque $[\mathrm{Nm}]$ & Average Torque $[\mathrm{Nm}]$ \\
\hline Torso Roll & 7.46 & 2.36 \\
Hip Pitch & 19.7 & 6.5 \\
Hip Roll & 44.8 & 16.45 \\
Hip Yaw & 14.95 & 4.27 \\
Knee Pitch & 38.0 & 10.6 \\
Foot Roll & 31.1 & 7.98 \\
Foot Pitch & 20.0 & 7.08 \\
\hline
\end{tabular}

The final step for the mechanical design was to complete a detailed design of all mechanical components. This included designing for machinability and an FEA analysis. The resulting design and completed biped are shown in Figures 2.5 and 2.6 . 


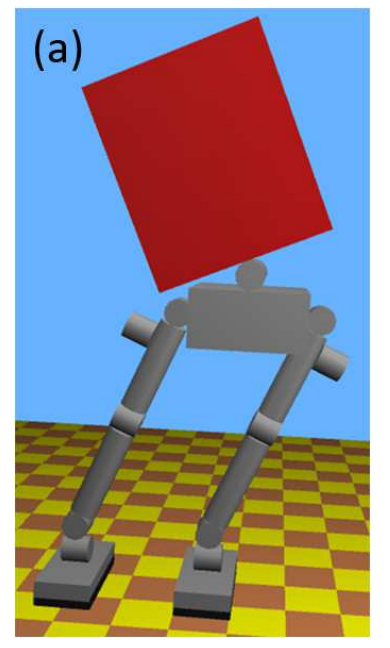

(b)

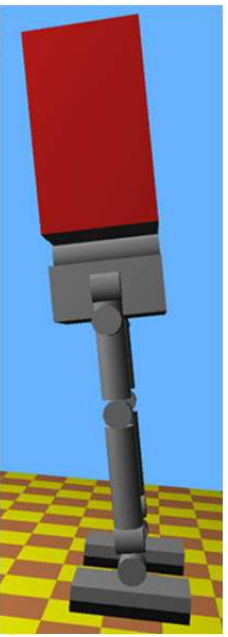

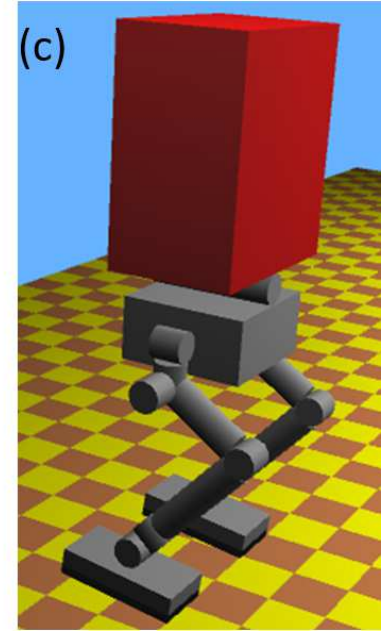

Figure 2.3: Different stances that were used to evaluate required joint torques: (a) maximum side weight shift, (b) maximum forward weight shift, and (c) crouched position.

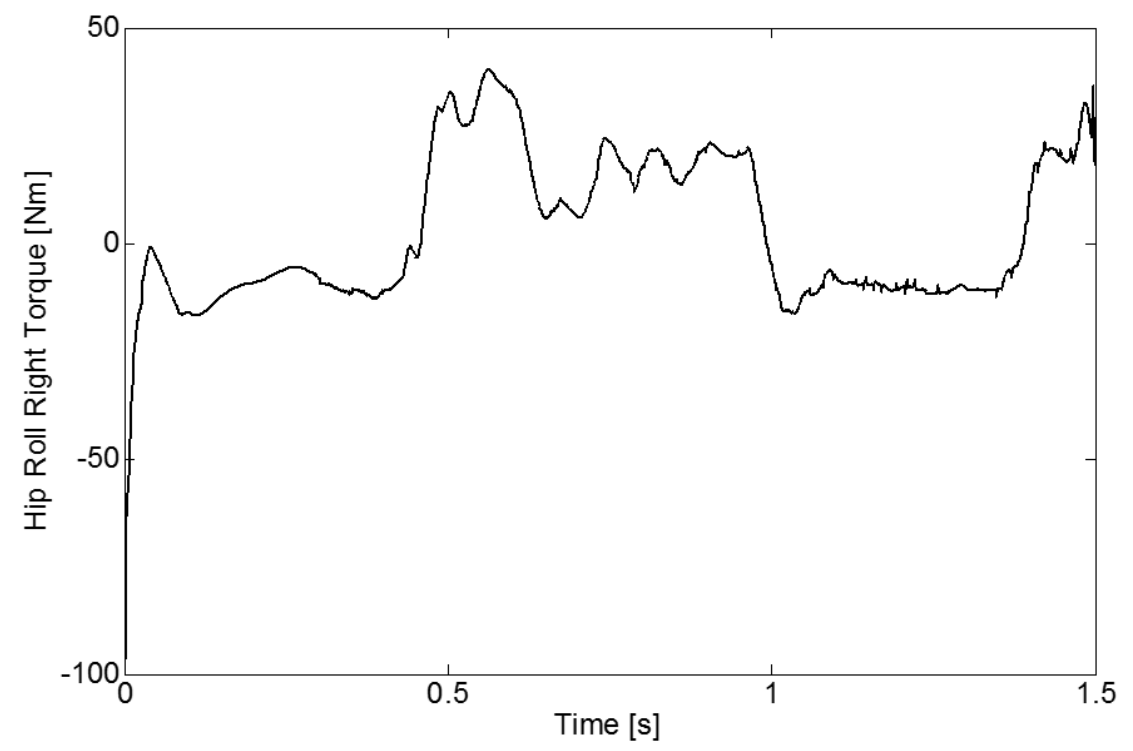

Figure 2.4: Example of resulting applied torque on the right hip roll joint to move the robot to the maximum side weight shift position (Figure 2.3(a)). 
Table 2.3: Selected actuation system for the ABL-BI design based on estimated torque requirements.

\begin{tabular}{r|l|l|l|l} 
Joint & Motor & Gear & Gear Reduction & Output Torque [Nm] \\
\hline Hip Roll & Maxon RE-40 & CSG171202UH & 120 & 44.2 \\
Hip Yaw & Maxon RE-30 & CSF141002XHF & 100 & 15.1 \\
Hip Pitch & Maxon RE-40 & CSG171202UH & 120 & 44.2 \\
Knee Pitch & Maxon RE-40 & CSG171202UH & 120 & 44.2 \\
Foot Roll & Maxon RE-30 & CSF141002XHF & 100 & 15.1 \\
Foot Pitch & Maxon RE-30 & CSF141002XHF & 100 & 15.1
\end{tabular}

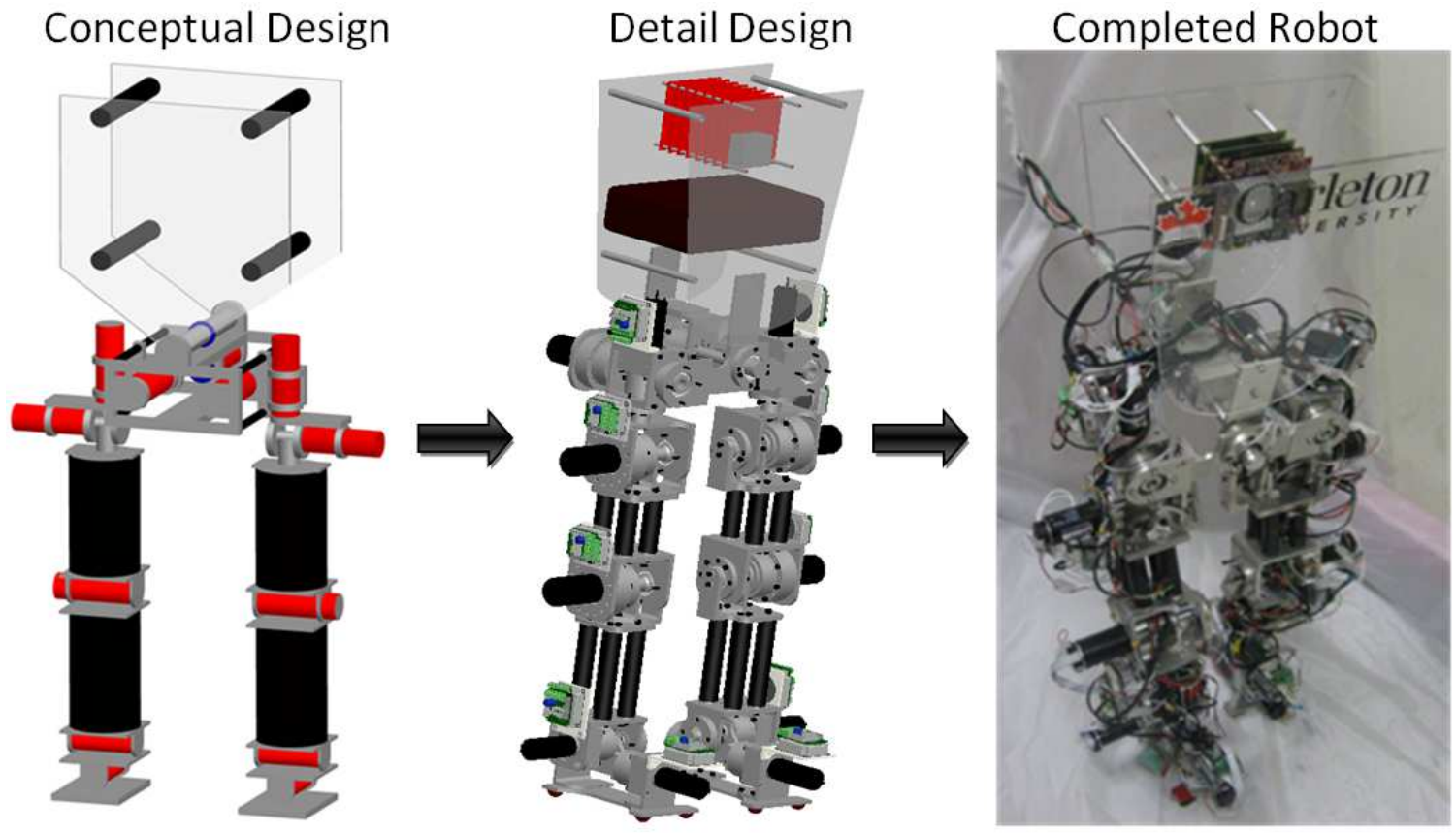

Figure 2.5: Progression of ABL-BI design from concept to detailed mechanical design and completed assembly. 


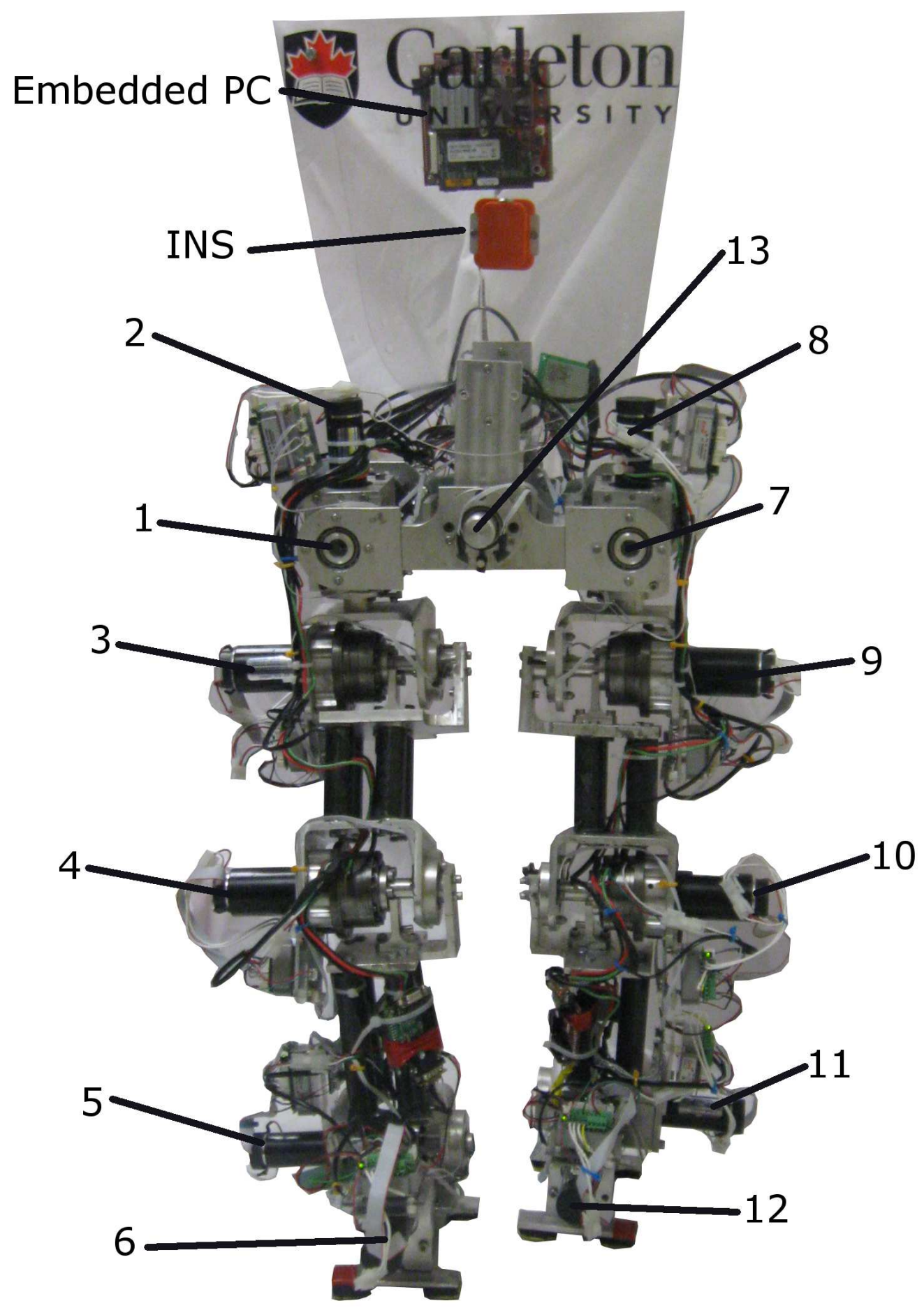

Figure 2.6: ABL-BI with associated joint numbers as defined in Table 2.4 


\subsubsection{Communications and Control Architecture}

The communications and control architecture was driven by two primary requirements: (1) real-time performance with control rates up to $500 \mathrm{hz}$ and (2) limiting the amount of wiring. This resulted in the selection of a distributed control architecture as shown in Figure 2.7. In this architecture local joint drives perform velocity, position or torque control of the motor. These drives also perform homing operations, check hall effect joint limit sensors as well as set current and velocity limits internally. Higher level control tasks are performed on an onboard embedded PC (Cheetah EMP-32 from Versalogic) or off-board control PC which can be connected to the network. The embedded PC communicates to the drives via a distributed CAN (controller area network) bus. Given the bandwidth limitations of the CAN bus ( $1 \mathrm{mbits} / \mathrm{s}), 2$ separate CAN buses share the total communication load. Joint position data is gathered by encoders on each motor and communicated back to the embedded PC via the CAN bus. Additional sensors include 8 Tekscan ${ }^{\circledR} \quad$ Flexiforce ${ }^{\circledR}$ sensors which provide a measurement of the normal force at the corners of each foot and an inertial navigation system (INS) in the torso which is used to measure the torso orientation. Overall, this architecture allowed for reduced wiring compared to a central, analog controlled architecture, increased robustness to noise and modularity, given that additional devices could easily be added or removed from the bus.

\subsubsection{Software Architecture}

The software architecture was co-developed with Owain Barker and presented in [113]. The primary requirements for the software architecture were (1) the ability to implement different control strategies and (2) the ability to easily integrate changes in hardware such as addition or removal of actuators and sensors. The software architecture must also provide a robust, real-time platform for the control system. Hence, a real-time operating system is necessary to implement the required scalable architecture. 

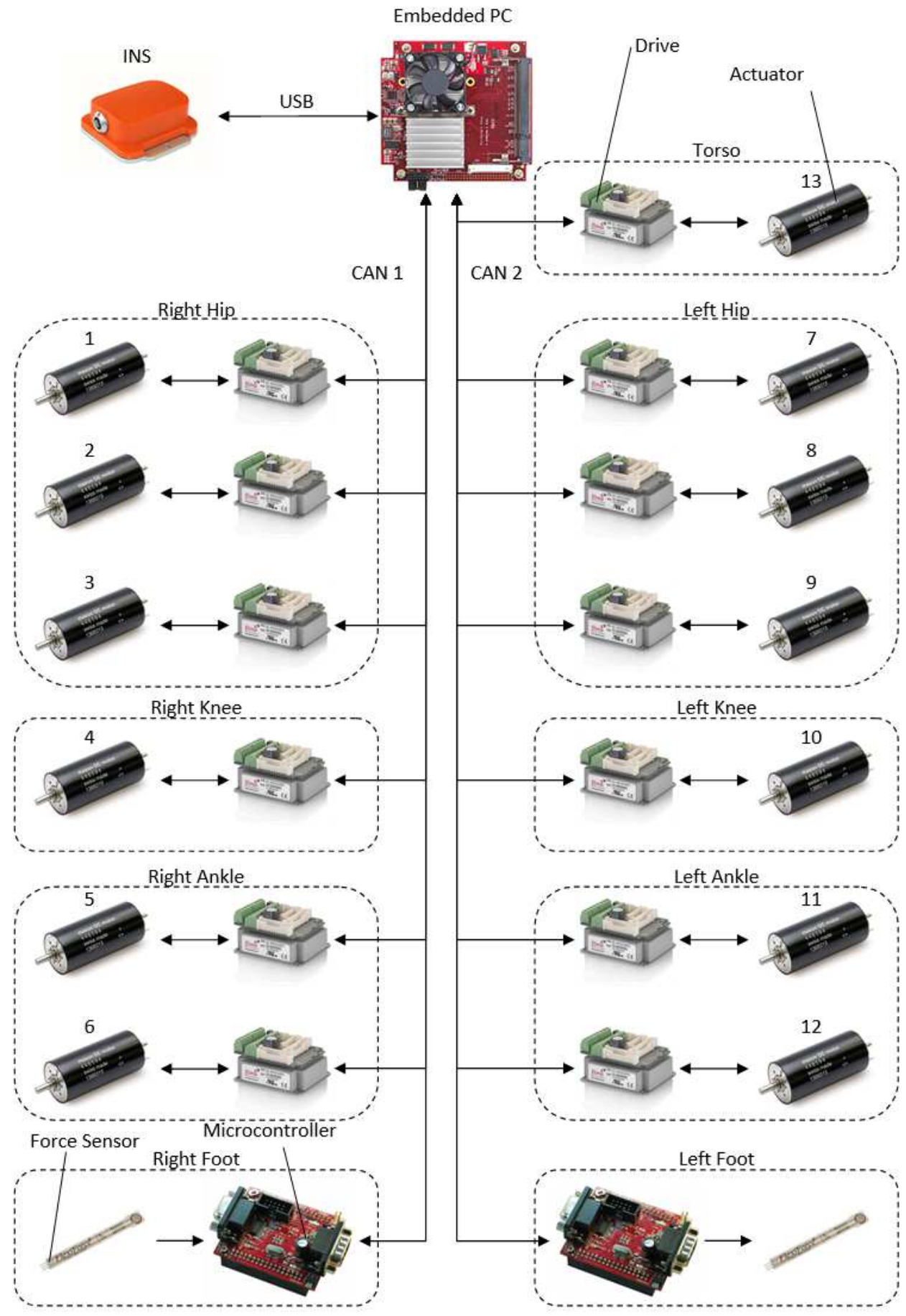

Figure 2.7: Communication and control architecture for ABL-BI. Control is implemented on a Cheetah EMP-32 embedded PC which communicates velocity commands via two CAN busses to Elmo Whistle motor drives. The drives implement a local velocity control loop with encoder feedback from motors (Maxon RE-30 or RE-40) and implement joint limits locally. 


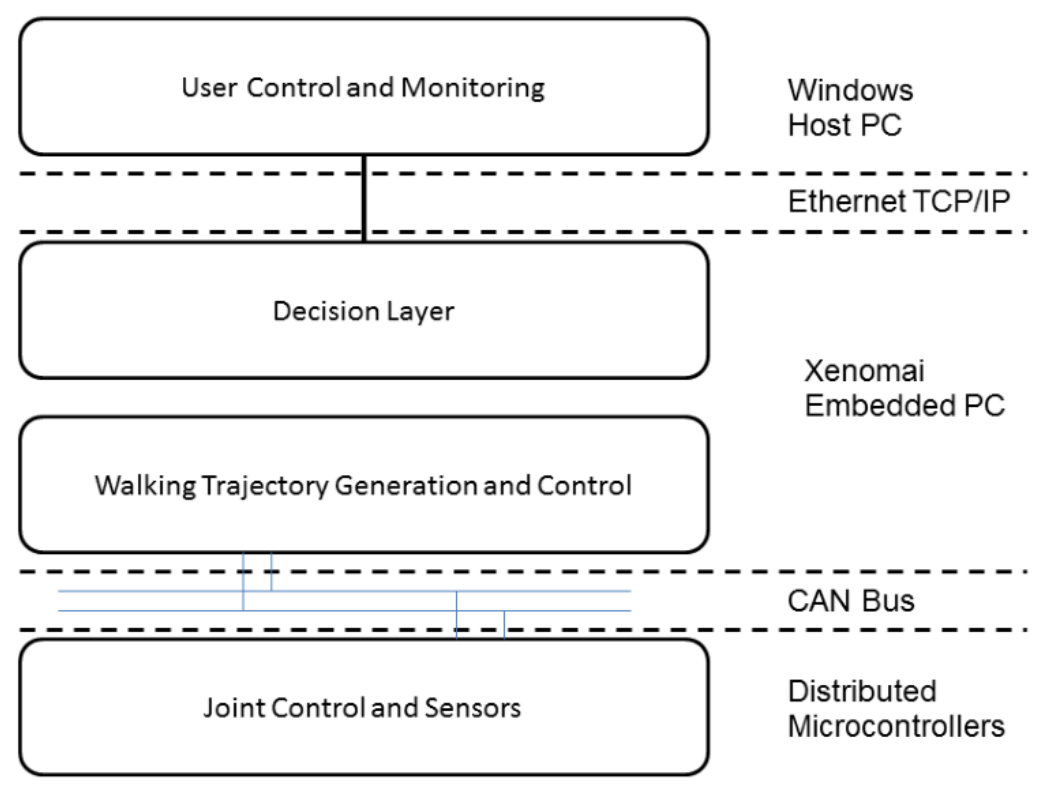

Figure 2.8: Software layers in ABL-BI control architecture.

\section{Real-Time Operating System}

The selection of the real-time operating system was based on real-time performance, hardware compatibility, cost, support and availability. Based on initial studies done and available comparative literature [114], Xenomai Linux was chosen. Xenomai is a branch that splits off from the RealTime Application Interface for Linux (RTAI) project. The performance achieved in both delay and jitter [114] of Xenomai, as well as the availability, and online community made it the choice for the embedded controller PC for ABL-BI.

\section{Software Design}

The software architecture includes four conceptual layers as illustrated in Figure 2.8. The layers are User Control and Monitoring, Decision, Walking Trajectory Generation and Control, and Joint Control and Sensors. These four layers may include one or multiple threads and span across three major device groupings; Client PC (Windows user station), embedded controller (Linux Xenmoai-based embedded computer), and distributed microcontrollers (joint control and sensors). 
The user control and monitoring encapsulates all the functionality for the user to interact, test, and run the hardware. This layer has no real-time requirements. The specific design requirements include; scalable and reconfigurable data logging and display, detailed error display, diagnostic tools, and initialization and runtime user interface. The decision layer operates on top of the real-time layer. This layer is responsible for the high-level decisions related to the overall control of the biped including: navigation through the environment, collision detection, user inputs, and data logging. This layer runs in the real-time kernel but is given the lowest priority.

The walking trajectory generation and control layer is the hard real-time portion of the system. Strict timing enforcement must maintain a constant control loop time period set by the user. The software architecture allows for sampling rates in excess of $1 \mathrm{KHz}$, but the control loop frequency is limited to $500 \mathrm{~Hz}$, due to the limited speed of operation of ELMO-Whistle drives on the CAN network. The trajectory generation, as well as the low level (joint-level) control loops are within this layer. Furthermore, the real-time network communication is handled within this layer. This provides the communication link between the embedded controller and all the actuators and sensors within the system. In total, the layer has two separate processes which contain a total of three separate threads.

The joint control and sensors layer is handled by the distributed hardware (within the Elmo drives). This layer is also hard real-time. The distributed microcontrollers interface with the actuator and sensor hardware, interpret the signals, and send the information at a user defined rate back to the embedded PC via the CAN bus. The Walking Trajectory Generation and Control layer coupled with the Joint Control and Sensors layer implements a feedback control loop across a digital bus.

\section{Software Architecture for the Embedded Controller}

The embedded controller encapsulates the decision layer and the Control and Trajectory layer shown in Figure 2.8. The structure for these layers is shown in Figure 2.9. The task 


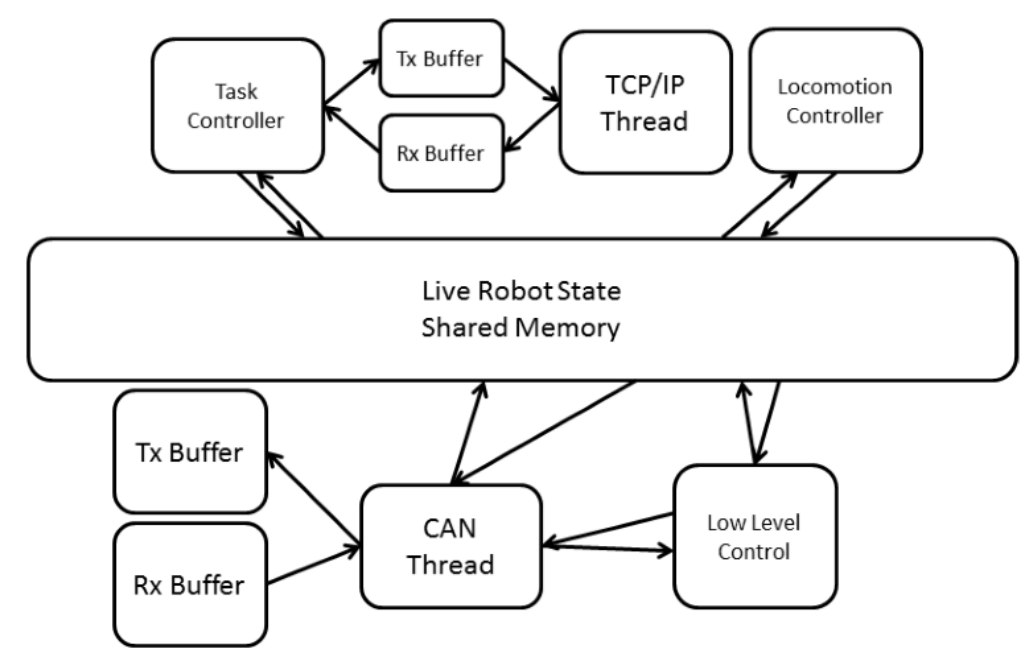

Figure 2.9: Architecture for individual software layers.

controller is the implementation of the decision layer. The requirements of this module are: communicate with the user interface via the TCP/IP thread, generate task-level control trajectories (such as COM velocity), and facilitate data logging. This layer is the lowest priority as the tasks it performs have soft deadlines.

The locomotion controller provides the modularity for plugging in the control strategies. There are two major subsections within this thread. The first is the supervisory controller, which is a state machine determining the current state of the robot. The number of states which can exist within the envelope of operation depends on the control strategy being employed. The second section is the trajectory planner. This is where the stabilizing walking control strategies which generate the joint trajectories are implemented. Finally the low-level control thread implements the network communication and performs the low level control loop to control the position of the joints. The low level controller receives all the sensor data from the CAN bus, the desired trajectories from the locomotion controller and computes the desired torque output. The output is sent to the ELMO-Whistle drives which provide the required current to the actuator.

In total, the architecture for the software has five threads, two for communication and 
three for control of the biped's motion. The current state of the robot as well as any information about past states is stored in shared memory. Each thread has access to the shared memory where strict access control is maintained. Given this layered, modular design, different control strategies can be tested quickly as long as they are in the form of $\mathrm{C}++$ functions. Also given that they are operating with the real-time framework of the Xenomai operating system, control of the robot is performed in a hard real-time manner.

\subsubsection{Kinematics and State Machine}

There are two core tools that must be developed for any bipedal robot controller: (1) a walking state machine and (2) forward and reverse kinematics. The kinematics are used in the controller to control biped motions such as the leg swing and COM shift. The walking state machine's primary objective is to identify which phase of walking the robot is in. This state is then used in the controller to plan the walking motion accordingly.

\section{State machine}

For the controllers used in this work, walking is separated in six walking phases as shown in Figure 2.10 and they are defined as follows:

- Double stance right (DSR): Phase of walking where both feet are on the ground, the right foot is the support foot and the left foot will be swinging.

- Double stance shift right (DSRS): Phase of walking where both feet are on the ground and the ZMP is located in support polygon of the right foot.

- Single stance right (SSR): Phase of walking where only the right foot is on the ground and the left foot is swinging.

- Double stance left (DSL): Phase of walking where both feet are on the ground, the left foot is the support foot and the right foot will be swinging. 


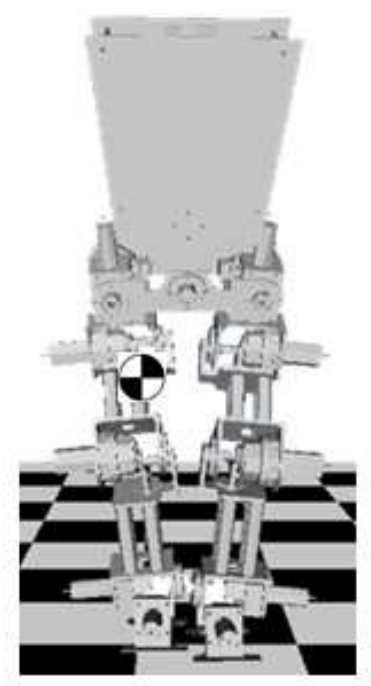

1-DSL

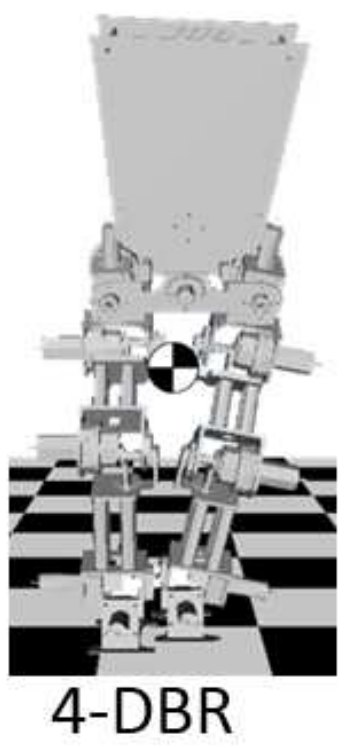

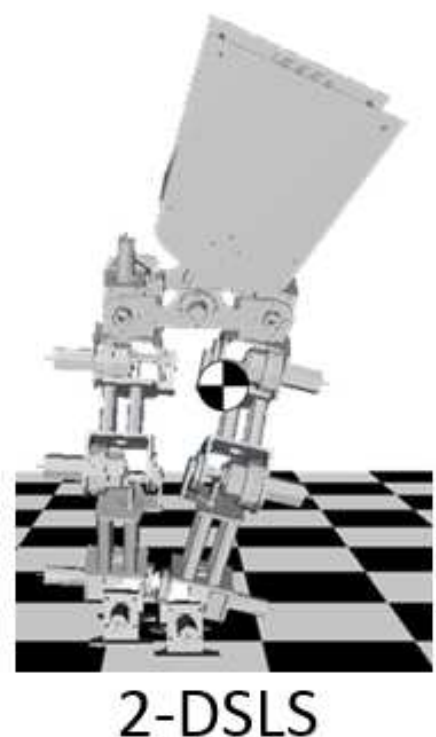

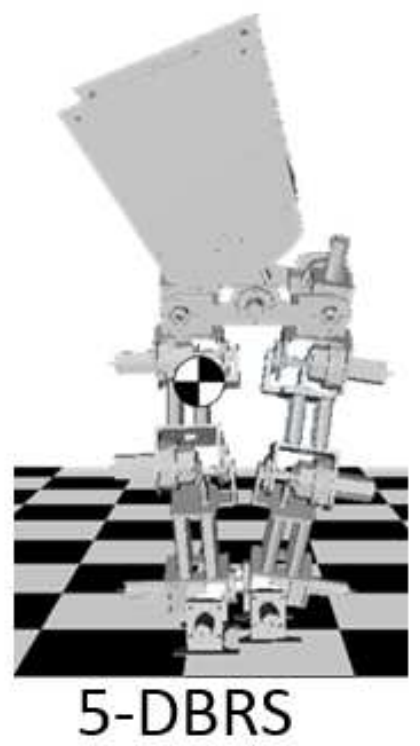

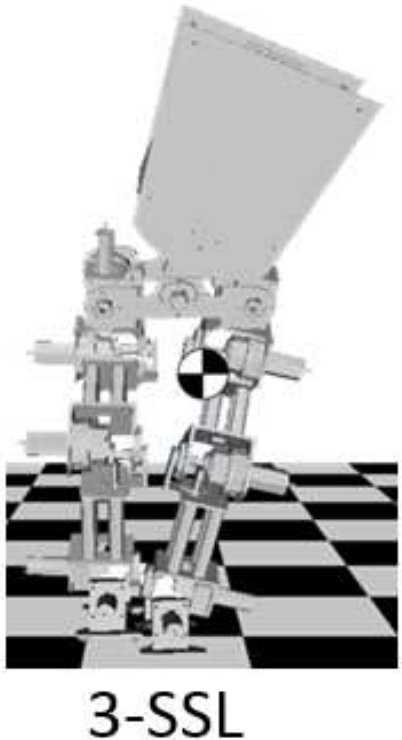

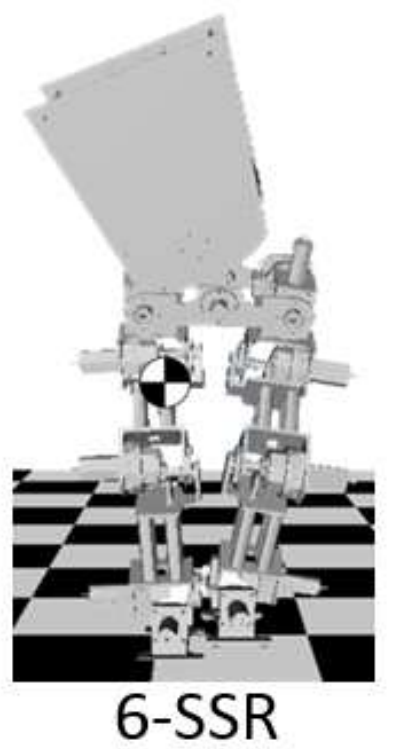

Figure 2.10: The different states of walking used in locomotion planning and control. 
- Double stance shift left (DSLS): Phase of walking where both feet are on the ground and the ZMP is located in support polygon of the left foot.

- Single stance left (SSL): Phase of walking where only the left foot is on the ground and the right foot is swinging.

During walking, the states will cycle through the different phases of walking as shown in Figure 2.10. The control system determines which state the robot is in by using the foot force sensors located on the bottom of the feet. The conditions for state transition are:

- DSR to DSRS: The ZMP or CMP (depending on the controller being used) must move within the support polygon of the right foot (stance foot).

- DSRS to SSR: The left foot begins to swing and ceases to be in contact with the ground, leaving only the right foot in contact with the ground.

- SSR to DSL: The left foot lands entering in contact with the ground.

- DSL to DSLS: The ZMP or CMP (depending on the controller being used) must move within the support polygon of the left foot (stance foot).

- DSLS to SSL: The right foot begins to swing and ceases to be in contact with the ground, leaving only the left foot in contact with the ground.

- SSL to DSR: The right foot lands entering in contact with the ground.

\section{Kinematics}

The overall kinematic structure for ABL-BI is shown in Figure 2.11 and local joint definitions are defined in Figure 2.11 and Table 2.4. This scheme is not consistent with Denavit-Hartenberg parameters typically used for robotic kinematics. Rather, they are consistent with the convention used in Webots ${ }^{\mathrm{TM}}$, the software initially used to model ABL-BI.

When deriving the kinematics, a reference frame must be defined for the derivation of the forward kinematics. In bipedal control there are three common reference frames: (1) 


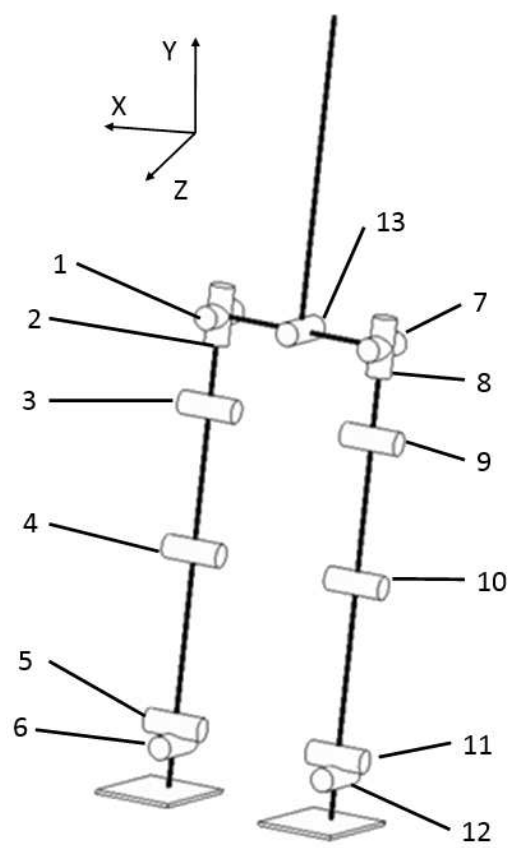

Figure 2.11: Kinematic configuration of the ABL-BI robot.

Table 2.4: Joint definitions and rotation axis.

\begin{tabular}{l|r|r} 
Joint Number & Joint Name & Axis of Rotation \\
\hline 1 & Hip Roll Right (HRR) & $\mathrm{Z}$ \\
2 & Hip Yaw Right (HYR) & $\mathrm{Y}$ \\
3 & Hip Pitch Right (HPR) & $\mathrm{X}$ \\
4 & Knee Pitch Right (KRR) & $\mathrm{X}$ \\
5 & Foot Pitch Right (FPR) & $\mathrm{X}$ \\
6 & Foot Roll Right (FRR) & $\mathrm{Z}$ \\
7 & Hip Roll Left (HRL) & $\mathrm{Z}$ \\
8 & Hip Yaw Left (HYL) \\
9 & Hip Pitch Left (HPL) & $\mathrm{Y}$ \\
10 & Knee Pitch Left (KPL) & $\mathrm{X}$ \\
11 & Foot Pitch Left (FPL) & $\mathrm{X}$ \\
12 & Foot Roll Left (FRL) & $\mathrm{X}$ \\
13 & Torso Roll (TR) & $\mathrm{Z}$ \\
&
\end{tabular}


the pelvis or body centred frame, (2) right foot frame or (3) left foot frame. Foot frames are used when the foot is in flat contact with the ground, in which case the foot reference frame acts as a ground-fixed reference frame. For each joint, the position of joint $i$ with respect to joint $j$ is denoted by $\vec{P}_{j}^{i}$ where $i, j=1,2, \ldots 13$. Similarly, the position of the COM of joint $i$ with respects to joint $j$ is denoted by $\vec{P}_{j}^{i C O M} \in \mathbb{R}^{4 X 1}$ where $i, j=1,2, \ldots 13$. The homogenous transformation matrix between two joint reference frames is denoted by $T_{j}^{i} \in \mathbb{R}^{4 X 4}$.

\section{Centre of Mass Position}

The position of the robot's COM is defined as

$$
\vec{P}_{j}^{C O M}=\frac{\sum_{i=1}^{i=13} m_{i} \vec{P}_{j}^{i}{ }_{\text {COM }}}{\sum_{i=1}^{i=13} m_{i}} j=6,12,13,
$$

where $m_{i}$ is the mass of link $i$ and $j$ is the selected reference frame which can be: (1) $j=6$ - right foot frame, (2) $j=12$ - left foot frame and (3) $j=13$ - pelvis frame. The velocity level kinematics can then be found by computing the derivative of (2.1) resulting in

$$
\dot{\vec{P}}_{j}^{C O M}=J_{j}^{C O M}(\vec{\theta}) \overrightarrow{\dot{\theta}} j=6,12,13
$$

where $J_{j}^{C O M} \epsilon \mathbb{R}^{3 X 13}$ is the Jacobian of the COM with respect to frame $j$ and is a function of the joint angles $\vec{\theta} \epsilon \mathbb{R}^{13}$. The inverse of this relationship can then be found as

$$
\overrightarrow{\dot{\theta}}=J_{j}^{\dagger C O M}(\vec{\theta}) \overrightarrow{\dot{P}}_{j}^{C O M} j=6,12,13,
$$

where, $J_{j}^{\dagger C O M}$ is the COM Jacobian pseudo-inverse with respect to joint $j$. Note that a complete list of kinematic parameters and joint homogeneous transformation matrices between joints is provided in Appendix A.

Swing Foot Position The second set of kinematics primarily required for control is defining the forward and reverse kinematics for the swing foot during single stance. These 
are used to control the position of the foot through a swing trajectory. In this case, the position of the swing foot will be found relative to the stance foot, which acts as the fixed reference frame during single stance. Therefore, if the right foot is the swing foot, we begin with the position of the right foot relative to the left foot frame as

$$
\vec{P}_{12}^{6}=T_{12}^{6} \vec{P}_{12}^{12}
$$

The velocity-level kinematics can then be derived by taking the derivative of (2.4) to get

$$
\overrightarrow{\dot{P}}_{12}^{6}=J_{12}^{6}(\vec{\theta}) \overrightarrow{\dot{\theta}}
$$

where $J_{12}^{6}$ is the Jacobian of joint 6 with respect to joint 12 . Similarly to the COM kinematics, the inverse relationship can be found as

$$
\overrightarrow{\dot{\theta}}=J_{12}^{\dagger 6}(\vec{\theta}) \overrightarrow{\dot{P}}_{12}^{6}
$$

Using these two sets of kinematic equations (COM position and swing leg position), basic control and motion planning operations for bipedal walking can be performed.

\subsection{Simulation Tools}

\subsubsection{D Dynamic Simulation}

The planar simulation consisted of a planar biped model as illustrated in Figure 2.12 with the model parameters listed in Table 2.5. This planar model is composed of 7 links with point

Table 2.5: Biped model specifications.

\begin{tabular}{lr}
\hline Height & $1.0[\mathrm{~m}]$ \\
Leg length & $0.6[\mathrm{~m}]$ \\
Torso length & $0.4[\mathrm{~m}]$ \\
Foot length & $0.15[\mathrm{~m}]$ \\
Total mass & $23[\mathrm{~kg}]$ \\
\hline
\end{tabular}




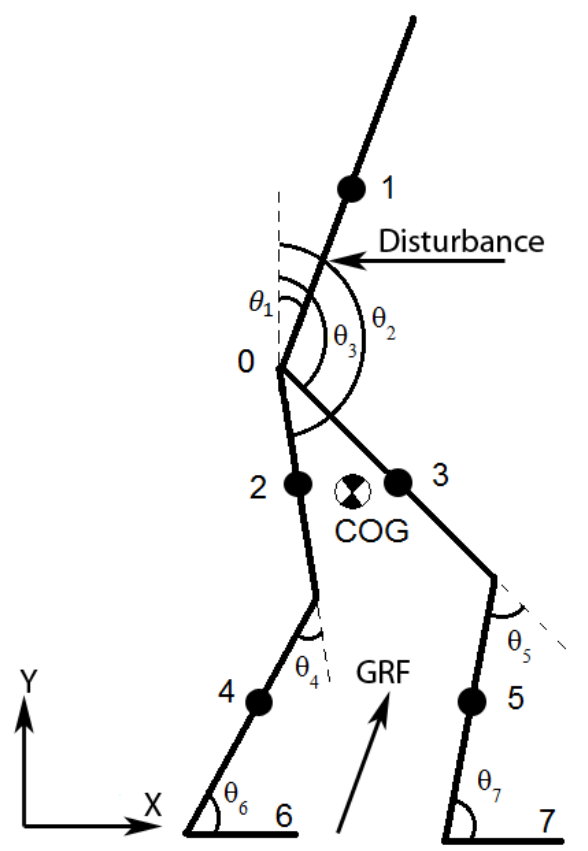

Figure 2.12: 2D planar biped model.

masses at the centre of each link. The model was built using Simulink ${ }^{\mathrm{TM}}$ SimMechanics $^{\mathrm{TM}}$ where each link is connected with a revolute joint. Joint angles are controlled via local joint PD controllers. Four contact points with the ground are defined, namely, forward and aft points of each foot. The normal ground reaction force is defined as a spring damper model as

$$
F_{N}=K_{g}\left(P_{y}\right)-D_{g}\left(\dot{P}_{y}\right)
$$

where $P_{y}$ is the height of the contact point (the ground height is considered to be the reference height), $K_{g}$ and $D_{g}$ are the ground stiffness and damping parameters. Similarly, the lateral ground reaction force is estimated using

$$
F_{L}=K_{g l}\left(P_{x}-P_{i x}\right)-D_{g l}\left(\dot{P}_{x}\right)
$$




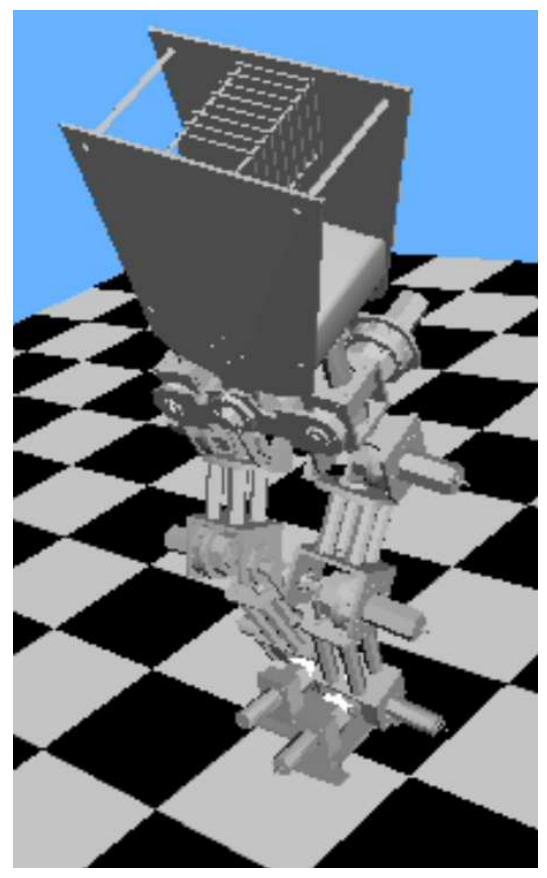

Figure 2.13: Visualization of the 3D dynamic simulation of ABL-BI.

where $P_{i x}$ is the initial contact point on touchdown of the foot with the ground and $P_{x}$ is the position of the contact point along the $\mathrm{X}$ axis. The lateral force can be limited to

$$
-\mu_{x} * F_{N}<F_{L}<\mu_{x} * F_{N}
$$

where $\mu_{x}$ is the friction coefficient. The parameters used for this model are listed in Table 2.6 ,

Table 2.6: Ground modelling parameters for planar biped simulation.

\begin{tabular}{lr}
\hline$K_{g}$ & $100000[\mathrm{~N} / \mathrm{m}]$ \\
$D_{g}$ & $500[\mathrm{Ns} / \mathrm{m}]$ \\
$K_{g l}$ & $1000[\mathrm{~N} / \mathrm{m}]$ \\
$D_{g l}$ & $500[\mathrm{Ns} / \mathrm{m}]$ \\
$\mu_{x}$ & 0.7 \\
\hline
\end{tabular}




\subsubsection{D Dynamic Simulation}

The 3D dynamic simulation was designed to emulate ABL-BI with a visualization shown in Figure 2.13. To achieve this, the kinematic structure of the model was designed to match the one in Figure 2.11. Link mass and inertia parameters were estimated from the detailed CAD model shown in Figure 2.5. Similarly to the planar model, the ground reaction force was estimated using a spring damper model as defined in Equations (2.8) and (2.9), where Equation (2.9) is also extended to the $\mathrm{Z}$ axis. The parameters for the ground model are the same as those listed in Table 2.6 .

\subsection{Chapter Summary}

In this chapter the design of ABL-BI, a 13 DOF biped was presented along with simulations tools that were built to develop and evaluate controllers. The design process consisted of setting requirements, a conceptual design which focused on actuator type and placement, and a detailed mechanical design using CAD tools.

Two separate simulation tools were developed for controller development: a planar biped simulation and a full 3D simulation meant to match ABL-BI's design. Both models were

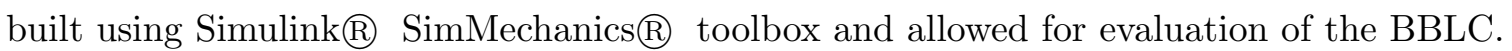




\section{Chapter 3}

\section{Behaviour-Based Locomotion Controller - Architecture and Implementation Examples}

As discussed in Chapter 1, one of the challenges with existing balance controllers is in adapting to disturbances that are not planned for a priori. This is a consequence of the primary assumption used in ZMP and other approaches as presented in Chapter 1. In these controllers, it is assumed that the robot is travelling on flat level ground. To adapt to steps or slopes, additional sensors and a modification to the controller are required [25, 26, 115]. Other approaches, such as using stepping manoeuvres or momentum regulation [38, 41, 43], have focussed on augmenting the robustness of the system to push disturbances. However, these approaches have not shown increased robustness to other disturbances the robot may encounter.

As a result, the principal motivation for the BBLC is to create a control architecture that is capable of generating new balancing strategies when faced with unknown disturbances. To achieve this objective, the BBLC controller employs a novel control architecture based on BBC. The core concept behind applying this architecture is that new balancing strategies will emerge from combining simpler balancing behaviours. To apply BBC architecture to locomotion problems, there are three core questions that must be answered and are the focus of this chapter: 
- Can the BBC architecture be augmented to redundant systems with multiple taskspace objectives, such as multi-legged robots?

- Can new balancing strategies emerge from combining multiple balancing behaviours?

- Is the novel BBLC strategy generalizable to robotic locomotion?

The generalized mathematical structure of the BBLC is presented in 3.1 and two demonstrative examples, mobile manipulator balance and bipedal robot balance, are presented in Sections 3.2 and 3.3 . The purpose of presenting these results is twofold: first to demonstrate the application of the BBLC, and second to show the generality of the BBLC by applying it to both legged and wheeled robotic platforms. Note that a more complete description and analysis of the BBLC will be presented in Chapter 4. Conclusions and a chapter summary follow in Section 3.4 .

\subsection{The Behaviour-Based Locomotion Controller - General Mathematical Formulation}

As discussed in Chapter 1, the concept of emergence is the principal behind the BBC architecture. However, previous uses of $\mathrm{BBC}$ were primarily applied to mobile robotic applications, which, compared to the proposed bipedal control problem, did not have redundancy or multiple task-space objectives. In this work, the BBLC augments the standard BBC architecture to accommodate systems with these two features. This is done by dividing the controller into three separate layers as illustrated in Figure 3.1 .

The Task Layer consists of the higher level motions a robotic system must achieve, such as position of an end effector in Cartesian space. For the two systems examined in this chapter, there are multiple task-space motions, each of which can be planned and controlled independently. For example, in a mobile manipulator, the motion of the mobile base and the motion of the manipulator can each be planned independently, as separate

task-space motions with a trajectory $\vec{X}_{r e f}$. Each motion must eventually be coordinated 


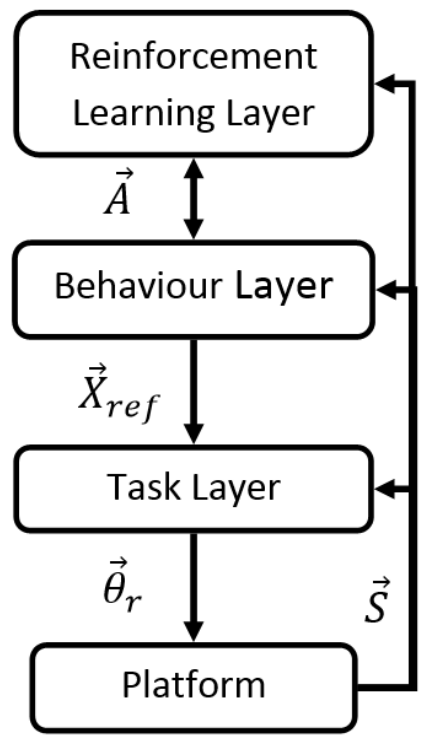

Figure 3.1: The BBLC architecture.

and decomposed into joint-space trajectories the system must track. The vector of jointspace trajectories, $\vec{\theta}_{r e f}$, is the output of the Task Layer sent to the physical system. These reference joint-space trajectories are then tracked by local joint controllers. The inputs into the Task Layer are the task-space trajectories planned in the Behaviour Layer. In this layer, behaviours consist of different methods of planning each task-space trajectory. Returning to the mobile manipulator example, two general planning behaviours can be defined for both the motion of the end effector and mobile base. A first set of behaviours which plan the trajectory of the end effector and mobile base to move the end effector to a defined position. A second set of behaviours can plan the motion to prevent tipping of the robot. For each task-space motion, the two different behaviours will plan a different trajectory. As a result, the system must select which behaviours to apply at a given time in the action vector $\vec{A}$, each element corresponds to a task-space motion and the entry to the element is an index corresponding to the behaviour being applied. Determining which combination of behaviours result in emergent balancing strategies is the role of the Reinforcement Learning Layer. In this layer, reinforcement learning is used to learn which behaviour should be applied for each task-space motion, given the current state of 


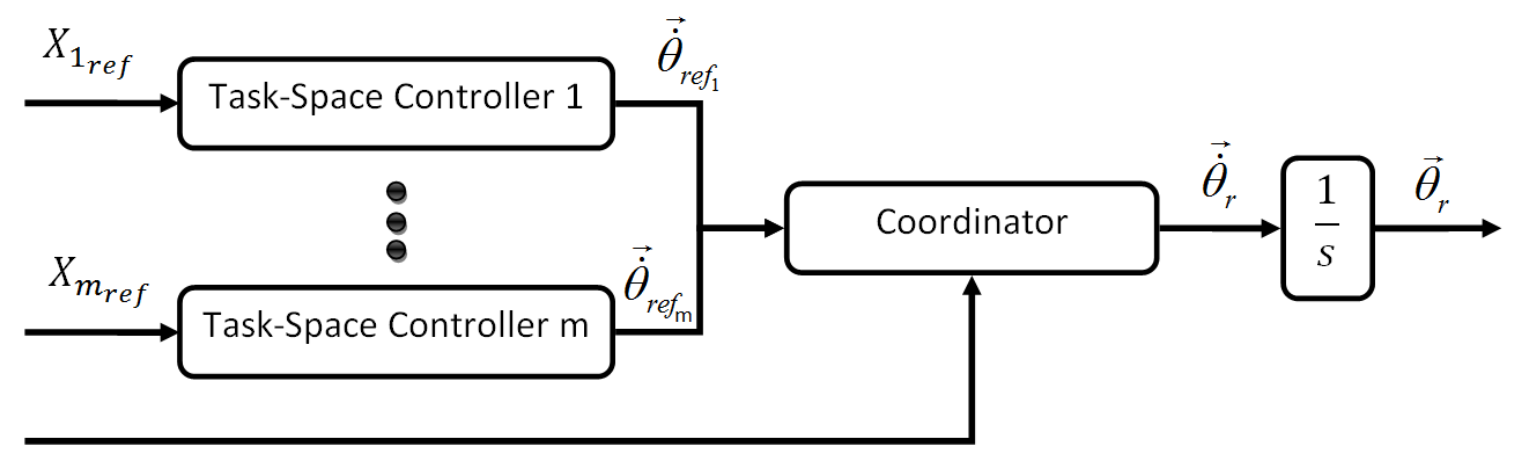

Task Priorities

Figure 3.2: The task layer combines the output for each task-space controller, with priority defined from the behaviour layer, to compute the final set of reference joint angles.

the system $\vec{S}$. As the system is exposed to more situations, it learns new combinations of behaviours that result in successful balancing strategies to particular disturbances. This gives a higher level understanding of each layer, Sections 3.1.1 to 3.1.3 will present each layer in more detail.

\subsubsection{Task layer}

Each task-space motion has a reference task-space trajectory, $\vec{X}_{i_{\text {ref }}} \in \mathbb{R}^{d_{i}} i=[1,2, . ., m]$, defined in the behaviour layer, where $d_{i}$ is the dimension of the task-space for a given task-space motion. The objective of the task layer is to find a set of reference joint-space trajectories, $\vec{\theta}_{r} \in \mathbb{R}^{n}$, that will track the reference task-space motion trajectories, where the joint-space consists of the controlled or actuated states of the system (e.g., the joint angles in a robotic arm) and $n$ is the dimension of the joint-space. The task layer divides the motion of the system into separate task-space controllers each having the objective of tracking one task-space trajectory. The division of the motion into separate modular controllers allows for different strategies to be planned for each task-space motion independently, as shown in Figure 3.2 . Once the trajectories $\vec{X}_{r e f}$ are planned in the behaviour layer, the objective of the task layer will be to generate reference joint-space trajectories, $\vec{\theta}_{r}$, that will coordinate the task-space motions together such that the reference task-space trajectories are tracked. A coordination method is required to combine the 
different task-space motions into a coherent final motion of the robot. A simple additive coordination, where the reference joint trajectories generated to track individual task-space trajectories are added together is unlikely to result in a coordinated motion where any task-space trajectories are followed. Conversely, a purely arbitrative coordination, where only one task-space trajectory is tracked at a time may result in an overly conservative approach, where certain task-space motions could have been followed concurrently. Hence in the BBLC, coordination is achieved by applying the task priority redundancy resolution algorithm [116]. In this algorithm, task-space motions are coordinated together based on priority. Lower priority tasks are projected into the null-space of higher priority tasks. This allows the higher priority task-space motions to be tracked unimpeded by the lower priority motions, while allowing the lower priority motions to also be tracked if there is sufficient redundancy in the system.

The first step is to define the inverse kinematics relationship at the velocity-level. The forward kinematics for a given task-space controller are defined as

$$
T: \theta \rightarrow X_{i} \quad i=[1, \ldots, m]
$$

where $X_{i} \in \mathbb{R}^{d_{i}}$ is the task-space position, $\vec{\theta} \in \mathbb{R}^{n}$ is the vector of joint angles, $i$ is the index of the task-space motion. Equation (3.1) can be differentiated to find the corresponding velocity level relationship for each task-space objective as

$$
\overrightarrow{\dot{X}}_{i}=J_{i} \overrightarrow{\dot{\theta}}
$$

where, $J_{i} \in \mathbb{R}^{d_{i} \times n}$, is the Jacobian. For the linear equation in 3.2 , the inverse relationship which uses the task-space velocities to find the reference joint velocities is

$$
\overrightarrow{\dot{\theta}}_{r e f_{i}}=J_{i}^{\dagger} \overrightarrow{\dot{X}}_{i}
$$

where $J_{i}^{\dagger}$ is the pseudoinverse of the Jacobian matrix and is non-square for redundant systems. The solution from (3.3) is a least-square solution with respects to the magnitude of $\overrightarrow{\dot{\theta}}_{\text {ref }}$. The task execution can be controlled in the task-space for a robot using simple 
linear controllers. For example, a PD controller can be used to define a reference velocity $\overrightarrow{\dot{X}}_{\text {iref }}$ as

$$
\overrightarrow{\dot{X}}_{i_{r e f}}=K_{P_{i}} \vec{e}_{i}-K_{D_{i}} \overrightarrow{\dot{e}}_{i}
$$

where $K_{P_{i}}, K_{D_{i}}$ are the proportional and derivative gains of the controller and $e_{i}$ is the error between the reference and actual task-space positions.

Once reference joint-space velocities are defined for each task-space controller, the task priority coordination method 116 can be used as a method of behaviour coordination [117, 118. The resulting equation that defines the final reference joint velocity is

$$
\overrightarrow{\dot{\theta}}_{r}=\overrightarrow{\dot{\theta}}_{r_{1}}+\left(I-J_{1}^{\dagger} J_{1}\right)\left[\overrightarrow{\dot{\theta}}_{r_{2}}+\ldots\left(I-J_{m-1}^{\dagger} J_{m-1}\right)\left(\overrightarrow{\dot{\theta}}_{r_{m}}\right)\right]
$$

where the subscripts $[1,2, \ldots, m]$ denote the priority of each individual task-space controller, 1 being the highest and $m$ the lowest. The priority assigned to each task-space layer is defined a priori in the design of each behaviour. The resulting reference velocities, $\overrightarrow{\dot{\theta}}_{r}$, consist of the solution that satisfies as many of the task-space controllers as possible, without compromising the highest priority controllers (where 1 corresponds to the highest priority). In other words, this algorithm allows for concurrent tracking of multiple task-space motions. Note that $\overrightarrow{\dot{\theta}}_{r_{i}}$ corresponds to the reference joint-space velocities for a priority level in 3.5 while $\overrightarrow{\dot{\theta}}_{r e f_{i}}$ corresponds to reference joint-space velocities for a task-space motion.

In summary, the key feature of the task layer is dividing the system's motion into multiple task-space controllers. This allows for a modular architecture, where the reference trajectory for each task-space controller can be planned independently in the behaviour layer. Additionally, using the task priority behaviour coordination method allows each task-space objective to be controlled individually, and its priority dynamically changed to accommodate the higher-level locomotion behaviours. 


\subsubsection{Behaviour Layer}

In the behaviour layer, behaviours are defined for each task-space motion, each defining

a different reference trajectory $\vec{X}_{r e f}$. Each behaviour corresponds to a different strategy or control objective for planning the task-space motion, e.g., for the motion planning of a biped's COM, ZMP or CMP based motion planning can be used. Each corresponds to a different method of planning the COM trajectory. Generally, each of these behaviours will be designed specifically for each application. In the BBLC, there is no optimization performed for individual behaviours; however, such an optimization can be performed independently to improve the performance of a specific behaviour. The selection of which behaviours are used is determined in the Reinforcement Learning Layer which outputs the action vector $\vec{A}$ to the Behaviour Layer. Given the behaviours selected in $\vec{A}$, the Behaviour Layer will output the set of reference task-space trajectories $\vec{X}_{r e f}$.

As described in Figure 3.3, for each task-space motion, multiple locomotion behaviours are defined, each generating a different trajectory, $\vec{X}_{i_{r e f}}$. For each task-space motion, only one behaviour can be selected at a time. The specific combination of different behaviours among the task-space motions consists of an emergent, higher-level stabilization strategy that can be applied to stabilize the system, where the total number of possible combinations is

$$
\prod_{i=1}^{i=m} b_{i}
$$

and $b_{i}$ is the number of behaviours for the $i^{\text {th }}$ task-space motion. To identify which behaviour is selected for a task-space motion, each behaviour can be given an index number that identifies the behaviour. This will result in $m$ elements, each containing an index for a task-space motion, that form the action vector $\vec{A} \in \mathbb{R}^{m}$. Hence, each action vector represents a unique combination of behaviours that can be applied to the system.

In addition to arbitrating between the different behaviours, a priority must be assigned to each task-space controller by the designer for the null-space coordination algorithm that 


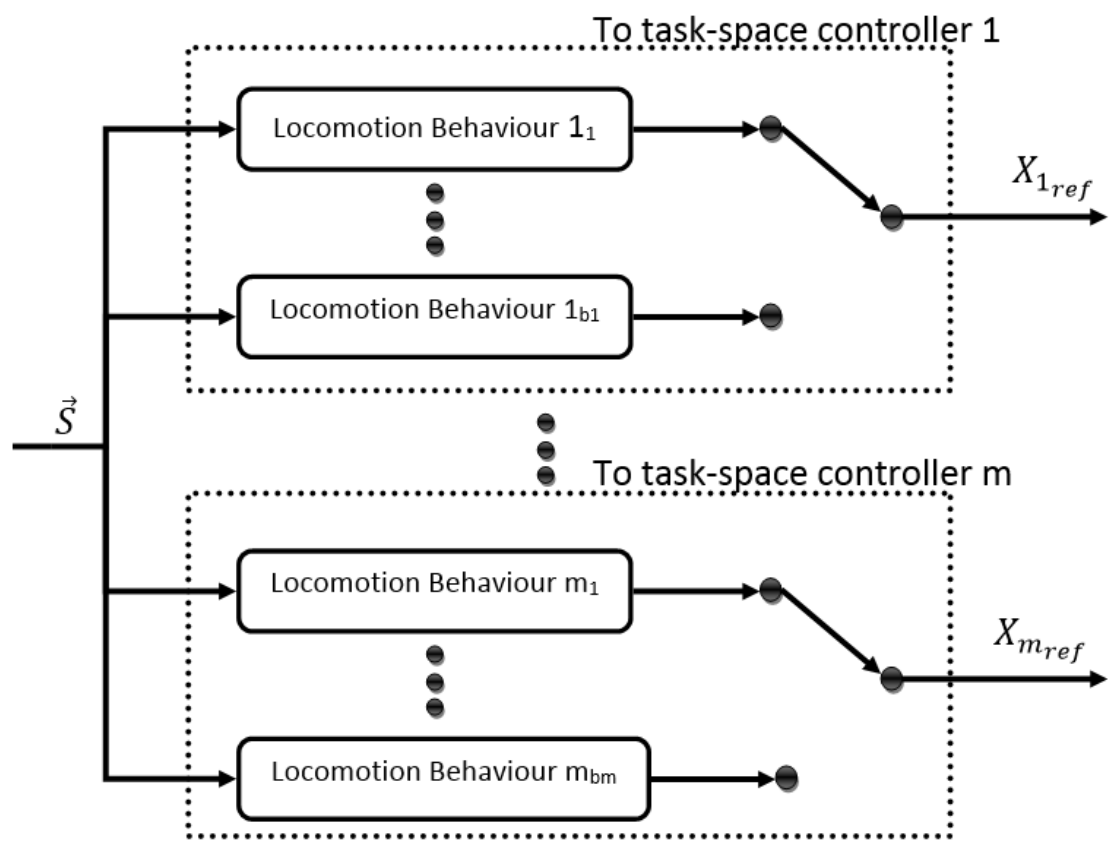

Figure 3.3: The behaviour layer has multiple locomotion behaviours which independently generate a reference task-space trajectory for each task-space motion. For each task-space motion, the trajectory from one locomotion behaviour is selected. A learning algorithm is used to determine which behaviour combinations lead to stabilization in the presence of disturbances.

is applied in the task layer. This priority is set in each behaviour. If a priority conflict occurs between two behaviours, the task-space controller with the smaller index is given priority (i.e., task 1 has the a higher priority than task 2 in case of a conflict). Finally, a given task-space controller can be excluded from the motion by assigning a priority outside the range of the coordination algorithm defined in $(3.5)$.

\subsubsection{Reinforcement Learning Layer}

The architecture of the behaviour layer and the task layer allow for different behaviours to be used for each task-space motion. Each behaviour combination represents a different strategy that can be applied to move and stabilize the robot. Given the large number of potential behaviour combinations, the challenges are (1) to find which combinations of behaviours result in successful emergent balancing strategies, and (2) for which disturbances they are applicable. In the BBLC, a reinforcement learning algorithm is used to learn which 
strategies can balance the robot when a specific disturbance is applied. Therefore, the objective of the reinforcement learning algorithm is to classify the disturbance that is applied to the system and determine which stabilization strategy is most likely to stabilize the robot. Classifying the disturbance has been studied previously in reactive-based approaches. In his work on bipedal control, Zaier classified the disturbance using sensors to detect steps or obstacles, and monitored the torso angle to detect push-type disturbances [2, 119, 120]. A more general classification approach has been studied to determine whether the robot is falling or not. Höhn and Gerth used a statistical classification, based on the robot's states, to determine whether the robot was falling and which fall preparation behaviour should be applied 121]. Similarly, Kalyanakrishnan and Goswami applied a supervised learning algorithm to classify whether the robot is falling or not 122 . However, rather than using the robot's states, composed of joint angles and joint angular velocities, they use a reduced state-space, $\bar{s}$, composed of stability relevant parameters, such as the linear and angular momenta and the contact state to predict stability. This approach allows for a reduced state-space that can be used to classify whether the robot is being disturbed or not.

In the BBLC, similar to the approaches above, a learning algorithm is used to determine which states corresponds to balanced or unbalanced conditions. Additionally, unlike in previous approaches, the learning algorithm also searches for an action vector $\vec{A}$ which can compensate for the unbalanced state. To classify the disturbance applied to the robot, a similar reduced state-space can be used for disturbance classification in the BBLC, where, $\bar{s}$, will vary as a function of the disturbances being considered as well as the application. Once a disturbance is classified, the learning algorithm must then find a behaviour combination capable of maintaining the robot's balance. In other words, in this learning problem an action vector $\vec{A}$ must be paired with an area in the state-space $\bar{s}$ in which $\vec{A}$ will successfully keep the system balanced. Additionally, the states causing a fall are distributed in time, i.e., the states causing a fall occur at some time before the fall. This is referred to as the state assignment problem, where it is unclear which conditions caused the current state of the robot. To address all of these conditions, learning is achieved by applying a Q-learning 
algorithm with eligibility traces $99,123,124$. The Q-learning algorithm $[99$ is defined as

$$
\begin{aligned}
& \underbrace{Q\left(\bar{s}_{t}, \vec{A}_{t}\right)}_{\text {new } Q}:=\underbrace{Q\left(\bar{s}_{t}, \vec{A}_{t}\right)}_{\text {previous } Q}+\underbrace{\alpha_{t}}_{\text {learning rate }} \underbrace{\lambda\left(\bar{s}_{t}, \vec{A}_{t}\right)}_{\text {eligibility trace }} \\
& \times[\underbrace{R\left(\vec{S}_{t+1}\right)+\gamma \max _{\tilde{\mathrm{A}}} \mathrm{Q}\left(\overline{\mathrm{s}}_{\mathrm{t}+1}, \tilde{\mathrm{A}}_{\mathrm{t}+1}\right)}_{\text {learnt update }}-Q\left(\bar{s}_{t}, \vec{A}_{t}\right)] .
\end{aligned}
$$

The Q-Learning algorithm is generally applied to determine optimal action-policy pairs for finite Markov Decisions Processes, where no knowledge of the value function $Q$ can be estimated initially [99. This differentiates it from predictor-corrector approaches where an initial estimate of the value function can be made. The variables in the Q-Learning algorithm are:

Q-value - the Q-value $Q\left(\bar{s}_{t}, \vec{A}_{t}\right)$ is a measure of the expected reward of performing the action, $\vec{A}$, at state, $\bar{s}$, and time, $t$. The new $Q$ is the newly calculated expected reward after the learnt update in applied at time $t$ to the previous $Q$.

Learnt update - In the learnt update, the reward function, $R\left(\vec{S}_{t+1}\right)$, is used to evaluate whether the resulting state meets the control objective or not. The definition of the reward function will vary based on the system; e.g., if maintaining balance is the control objective, positive reward will be given when the robot is balanced and negative reward will be given when falling, or tip-over is detected.

Learning rate - The learning rate $\alpha_{t}=\left[\begin{array}{ll}0 & 1\end{array}\right)$ is a parameter which weighs the learnt update at time $t$. If $\alpha_{t}=0$, then no learning occurs and the previous and new Q-values will remain equivalent. As $\alpha_{t} \rightarrow 1$ then the learnt update is weighted in favour of the previous Q-value, i.e., the old Q-value is discarded in favour of a new value. In the BBLC $\alpha_{t}$ is kept constant with time; however, generally $\alpha_{t}$ can vary with time, and is typically kept constant for simplicity. Learning can also be divided into two phases where in a first phase $\alpha_{t} \approx 1$ for faster initial learning. In a second phase $\alpha_{t}$ is reduced to a lower value where the previous Q-Values are weighted higher and the learnt update acts to fine tune to current Q-Value rather than replace it.

Eligibility trace - The eligibility trace will apply the learnt update from time $t+1$ to the historical states, $t-i$ where $i=[1,2, \ldots, h]$, that preceded the current state, where 


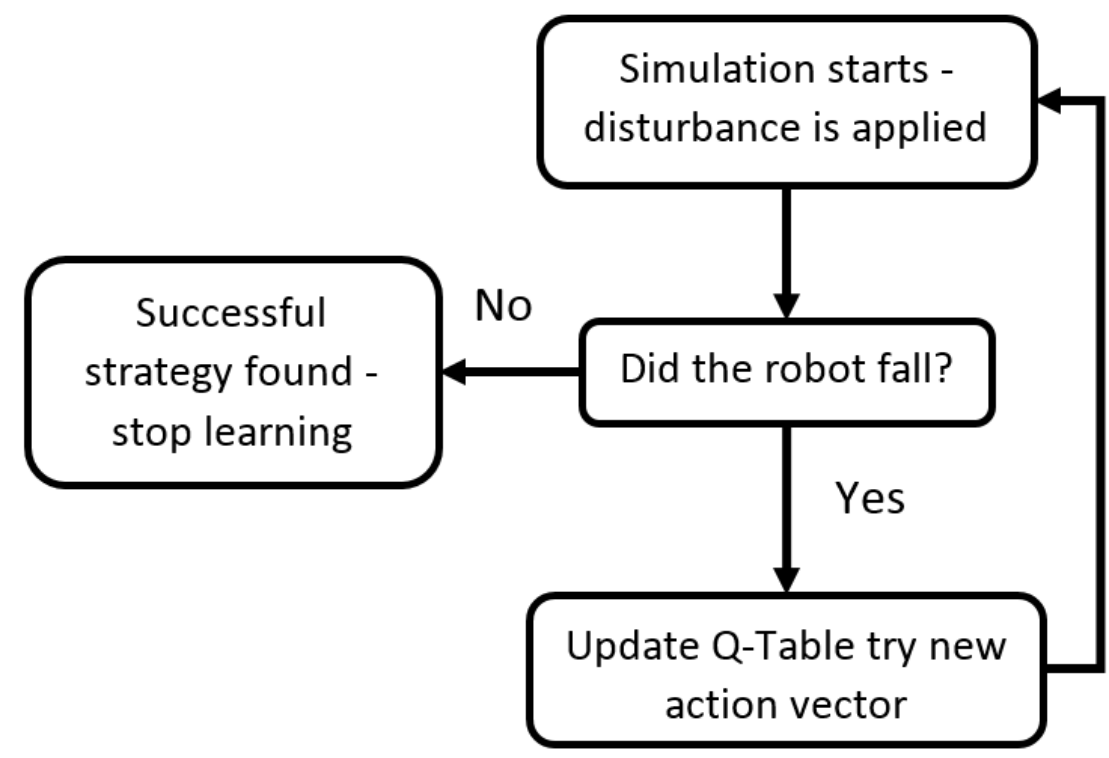

Figure 3.4: Flow chart describing the learning process as it occurs in simulation.

$h$ is the number of time steps kept in memory. The eligibility trace parameter $\lambda=\left[\begin{array}{ll}0 & 1\end{array}\right)$ weighs the learnt update for each historical state by $\lambda^{i}$. This approach is used to apply the learnt update to previous states as well, as these states may have contributed to the current reward; e.g., an action that occurred several seconds ago may have contributed to the current balance condition of the robot.

The learning process applied in simulation is summarized in the flow chart in Figure 3.4. Initially a disturbance is applied to the system where the BBLC applies the default action vector $\vec{A}=[11, \ldots, 1]^{T}$. If the system remains balanced, then the default strategy was sufficient to maintain balance and additional learning is not required. Conversely, if a fall occurs, negative reward will be applied using the algorithm described in (3.7). The simulation will then repeat with the same disturbance condition, and a new action vector $\vec{A}=[11, \ldots, 2]^{T}$ will be applied as a result of the negative Q-value assigned in the previous learning iteration to $Q\left(\bar{s}_{t}, \vec{A}_{t}\right)$. This iterative process will continue until an action vector resulting in a balanced system is found or until all possible action vectors have been attempted where the final action vector is $\vec{A}=\left[b_{1} b_{2}, \ldots, b_{m}\right]^{T}$. After learning is completed, 
if the system enters the same state-space in $\bar{s}$ as in the learnt case, the controller will activate the new balancing strategy, as it will have accumulated positive reward, compared to negative reward for other unsuccessful strategies. The resulting Q-function can be used in the control of the actual system without performing additional learning. This allows for unsafe learning, where failure may be common, to occur in simulation only.

\subsection{Mobile Manipulator Balance Control}

The BBLC is implemented on a mobile manipulator to demonstrate its application through a simpler example, where balance is still an important control objective. Mobile manipulators are an application where maintaining balance becomes challenging when large loads, compared to the mass of the mobile base, are being manipulated. For most applications, the manipulator has a workspace that extends beyond the support polygon of the mobile base 125 . If a sufficiently large load is being manipulated, the robot can tip over, causing the system to fail. Also, travelling on sloped or rough terrain can cause tipping [126]. Similarly to the bipedal balance problem, current approaches to preventing tipping in mobile manipulator balance have been based on the ZMP 125, 127,130. However, given that most mobile manipulators remain tele-operated, most efforts have been focused on designing tipping measures that estimate when the robot will tip, rather than designing automatic control strategies capable of continuously predicting and avoiding falls $126,131,135$.

The mobile manipulator example provides a first application of the BBLC. Maintaining balance of the system is important and there are multiple task-space motions that can be planned with various stabilization behaviours. The robot is modelled after a four-wheeled, car-like mobile base and a five DOF manipulator (all revolute) placed in the centre of the base, as shown in Figure 3.5. The link and model parameters are listed in Table 3.1 .

Two challenging test cases are examined: (1) a large load that can cause tipping is placed 


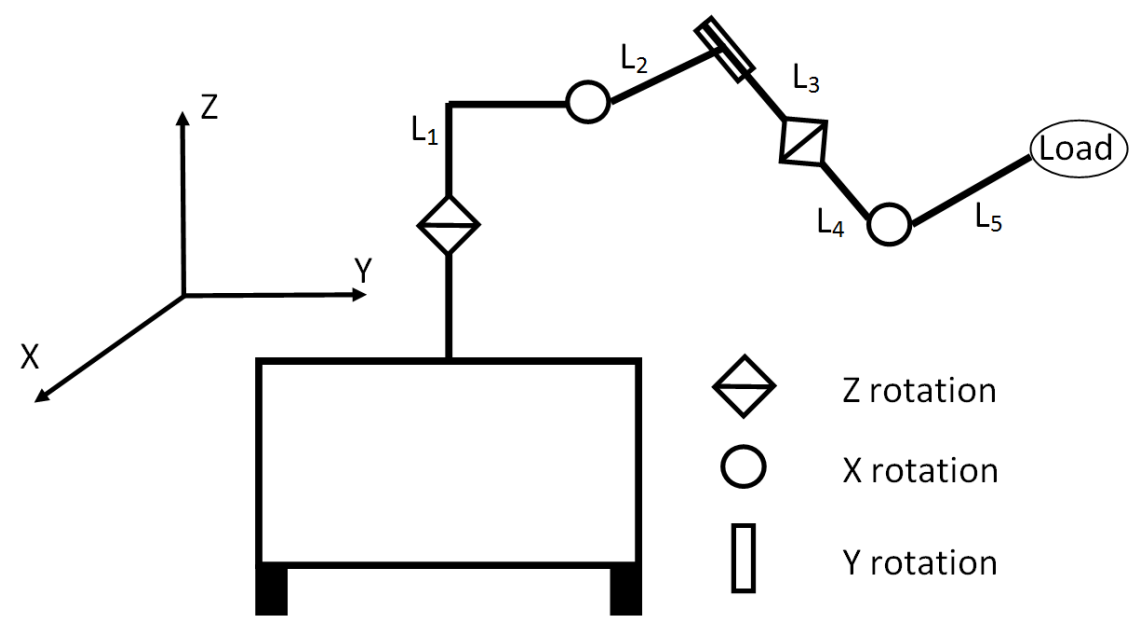

Figure 3.5: Mobile-manipulator dynamic model is composed of a four wheel, car like steering mobile base and a five DOF manipulator.

Table 3.1: Mobile manipulator model parameters.

\begin{tabular}{|l|l|l|l|l|}
\hline Link & $\mathrm{X}[\mathrm{m}]$ & $\mathrm{Y}[\mathrm{m}]$ & $\mathrm{Z}[\mathrm{m}]$ & mass $[\mathrm{kg}]$ \\
\hline \hline Base & 0.25 & 0.25 & 0.2 & 50 \\
L1 & 0 & 0.2 & 0.6 & 30 \\
L2 & 0 & 0 & 0 & 10 \\
L3 & 0 & 0 & 0 & 5 \\
L4 & 0 & 0 & 0.2 & 5 \\
L5 & 0 & 0.3 & 0 & 15 \\
\hline
\end{tabular}


at the end effector and (2) the robot is placed on a steep slope. The objective of presenting these two cases is to examine if the BBLC can generate new balancing strategies for two different disturbance conditions, while using the same bank of behaviours. The results for each case are presented in Section 3.2.4 preceded by a description of each controller layer in Sections 3.2 .1 to 3.2 .3 ,

\subsubsection{Task Layer}

The system must first be separated into multiple task-space motions for which different behaviours can be planned. For the mobile manipulator three task-space motions are defined as:

1. Positioning the end effector at a desired position in the Cartesian space $\vec{X}_{1} \in \mathbb{R}^{3}$;

2. Positioning the mobile base at a desired position $\vec{X}_{2} \in \mathbb{R}^{3}$;

3. Controlling the position of the COM of the robot $\vec{X}_{3} \in \mathbb{R}^{3}$.

For each task-space motion, a reference trajectory must be defined in the behaviour layer. As described in Section 3.1.1, for each task-space motion a velocity-level controller is defined with the corresponding Jacobian transformation relating each task-space velocity to the joint space. For the mobile manipulator, the joint-space consists of the manipulator joint angles, $\vec{\psi} \in \mathbb{R}^{5}$, and the mobile base pose, $\left[\begin{array}{lll}x & y & \rho\end{array}\right]^{t}$, as

$$
\vec{\theta}=\left[\vec{\psi}^{t} x y \rho\right]^{t}
$$

Given that there are three task-space motions, the resulting task priority equation as adapted from (3.5) is

$$
\overrightarrow{\dot{\theta}}_{r}=\overrightarrow{\dot{\theta}}_{r_{1}}+\left(I-J_{1}^{\dagger} J_{1}\right)\left[\overrightarrow{\dot{\theta}}_{r_{2}}+\left(I-J_{2}^{\dagger} J_{2}\right)\left(\overrightarrow{\dot{\theta}}_{3}\right)\right]
$$

where the priority of each task-space motion is defined in the behaviour layer. 


\subsubsection{Behaviour Layer}

The behaviour layer defines the trajectories for each task-space motion defined in the task layer. In this case three different behaviours for each task-space motion are defined, as listed in Table 3.2. As discussed previously, each behaviour plans the trajectory of a task-space motion to achieve a control objective, which in this example are either to position the end effector or to maintain balance. Each behaviour is planned independently to achieve one of these objectives.

Table 3.2: Behaviours for mobile manipulator.

\begin{tabular}{l}
\hline End effector position \\
\hline \hline 1-Track reference trajectory \\
2-Move end effector to centre of mobile base \\
3-Exclude end effector control \\
\hline \hline Mobile base control \\
\hline \hline 1-Move mobile base to reference position \\
2-Move base to position the ZMP towards the centre of the \\
support polygon \\
3-Exclude mobile base control \\
\hline \hline Centre of mass position \\
\hline \hline 1-Move COM to reference position \\
2-Generate COM motion to move ZMP to the \\
centre of the support polygon \\
3-Exclude COM position control
\end{tabular}

\section{Tipping Load}

When an "exclude behaviour" is applied, the given task-space motion is excluded for the reference task-space velocity computation in (3.9). For the reference tracking trajectory behaviours, a reference position is defined by the user and trajectory tracking is achieved through the inverse Jacobian control described in Section 3.1.1. The mobile base reference 
position control is achieved by applying a direct feedback linearization controller as defined in [136]. As stated in the introduction, many balance controllers are based on the ZMP criterion. The ZMP is defined as the point on the ground where the net GRF must act to balance the net moments acting on the system. Hence, a sufficient condition to maintain balance is for the ZMP to remain within the support polygon. Mobile base control behaviour 2, attempts to move the ZMP toward the centre of the support polygon by defining a reference base velocity as

$$
\left[\begin{array}{l}
\dot{x} \\
\dot{y}
\end{array}\right]=K_{2} \vec{e}_{Z M P},
$$

where, $\vec{e}_{Z M P}$, is

$$
\vec{e}_{Z M P}=\left[\begin{array}{c}
x_{Z M P} \\
y_{Z M P}
\end{array}\right]-\left[\begin{array}{l}
x_{0} \\
y_{0}
\end{array}\right] .
$$

$x_{Z M P}$ and $y_{Z M P}$ are the position of the ZMP, and $x_{0}$ and $y_{0}$ are the centre of the mobile manipulator.

\subsubsection{Reinforcement Learning Layer}

For the Q-learning algorithm, the reduced state-space, $\bar{s}$, must be defined, as well as a method of function approximation for the Q-function. For the mobile manipulator application, three states are used: (1) the stability margin $S_{m} \in \mathbb{R},(2)$ the Rate Of Change (ROC)

of the stability margin $\dot{S}_{m} \in \mathbb{R}$ and an estimate of the terrain slope angle $\lambda \epsilon \mathbb{R}$ with respect to the horizontal plane. The stability margin, $S_{m} \in \mathbb{R}$, defined as

$$
S_{m} \equiv \min _{\forall \vec{X}_{s p}}\left(\vec{X}_{s p}-\vec{X}_{z m p}\right)
$$

where, $\vec{X}_{s p}$ is a point on the support polygon. These states are selected to estimate the balance state of the robot based on their characteristics. The stability margin and its time derivative give a direct measure of how close the robot is to tipping while the slope estimate gives a measure of the magnitude of the slope the robot may be travelling on. This results in 
$\bar{s}=\left[S_{m} \dot{S}_{m} \lambda\right]^{T} \in \mathbb{R}^{3}$. Given this dimensionality, the Q-function can be approximated with a lookup table where $S_{m}, \dot{S}_{m}$ are discretized with 100 points with a range of [0 0.25$] \mathrm{m}$ and [-5 5] $\mathrm{m} / \mathrm{s}$ respectively. The terrain slope is discretized more coarsely, with 10 points and a range of [-50 50] degrees. This discretization keeps the size of the lookup table manageable from a computational perspective (a total of 100,000 different state combinations exist), without losing significant resolution of the state variables. The values for each cell in the Q-function lookup table are initialized to zero. The reward function used to evaluate the current state of the robot is defined as

$$
R \equiv\left\{\begin{aligned}
1 & \delta \leq 0.5[\mathrm{rad}] \\
-1 & \delta>0.5[\mathrm{rad}]
\end{aligned}\right.
$$

where $\delta$ is defined as

$$
\delta \equiv \max \left(\alpha_{\mathrm{x}}-\lambda, \alpha_{\mathrm{y}}-\lambda\right)
$$

is the maximum difference between the mobile base orientation and the ground slope and $\alpha_{x}, \alpha_{y}$ are the pitch and roll of the mobile base. Hence, a positive reward is given if the robot is not tipping and a negative reward is given if the robot has tipped beyond $0.5 \mathrm{rad}$.

\subsubsection{Experiments}

Two different conditions are examined in the mobile manipulator simulation: a first where an $85 \mathrm{~kg}$ load is placed on the end-effector and the end-effector position initially lies outside of the support polygon; a second where the mobile manipulator moves up a $40^{\circ}$ slope. Both these cases present conditions which can cause the robot to tip over and in both cases the controller is not aware of the disturbance acting on the system. The objective of both experiments is to examine if the BBLC will recognize the unbalanced state of the system and learn an emergent strategy to recover and maintain balance.

\section{Tipping Load}

In this experiment, a load is placed at the end effector where the mass of the load is not known by the controller. The initial configuration of the end effector extends beyond 
the support polygon as seen in Figure 3.6 at $0 \mathrm{~s}$ and the robot is initially at rest on flat level ground. For loads of $20 \mathrm{~kg}$ and above, if no action is taken this causes the robot to tip over. For the reference behaviour combination where $\vec{A}=\left[\begin{array}{lll}1 & 1 & 1\end{array}\right]^{T}$, the robot tracks a reference mobile base and end effector trajectory without considering the system's balance. Applying this behaviour combination, the Q-function in Figure 3.7 is obtained, where $\lambda=0^{\circ}$. For this function, it can be seen that for $S_{m}<0$ and $\dot{S}_{m}<-1$ the Qvalue becomes negative; indicating that under these conditions a tip is expected to occur, based on the learnt Q-function. The learning algorithm successfully finds several behaviour combinations which can maintain stability. As the load is increased to $85 \mathrm{~kg}$, only one stabilization strategy remains successful, where the behaviours used are $\vec{A}=\left[\begin{array}{lll}2 & 2 & 3\end{array}\right]^{T}$, resulting in the motion shown in Figure 3.6. Using this behaviour combination, the robot moves the end effector to the centre of the mobile base, effectively moving the COM toward the centre of the robot. Additionally, the robot applies the mobile base ZMP strategy described in Section 3.2.2, while excluding the control of the COM position. The resulting Q-function for this strategy, shown in Figure 3.8, indicates that in the same region where the reference behaviour Q-function was negative, the Q-value is positive, indicating that for similar states, balance was maintained. This result indicates the controller was able to identify an emergent balancing strategy from the set of behaviours provided, allowing it to maintain balance in the presence of the unknown disturbance. Additionally, although not presented in the results, for disturbances of a smaller magnitude, it was found that several successful strategies were found when an exhaustive search was performed. However, as the magnitude of the disturbance was increased, fewer strategies successfully maintained balance until only a single strategy (the one presented above) was able to maintain balance.

\section{Slope}

In this experiment, the robot travels up a $40^{\circ}$ slope at $1 \mathrm{~m} / \mathrm{s}$ and attempts a $90^{\circ}$ turn to move transversely across the slope with no payload placed on the end-effector. If no balancing strategy is used, the robot tips over as seen in Figure 3.9. The resulting Q-function for using 


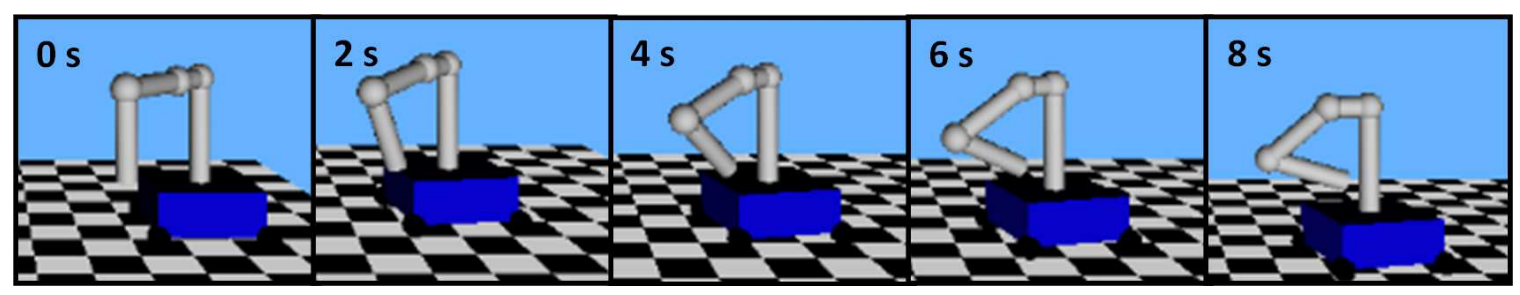

Figure 3.6: Mobile-manipulator recovering from a potential fall with $85 \mathrm{~kg}$ load at the end-effector.

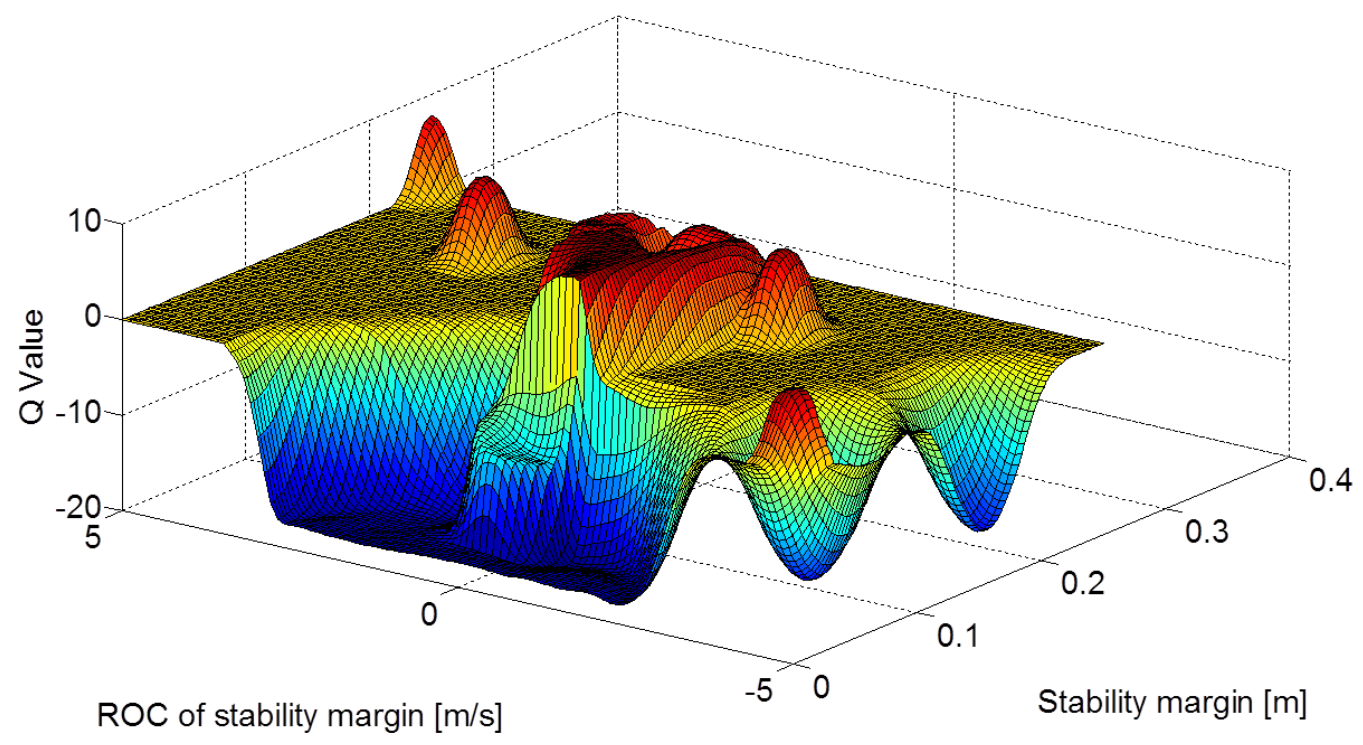

Figure 3.7: Q-function for the reference behaviour combination where the end-effector moves to a user defined position. Negative Q-values are accumulated when this behaviour combination is unable to compensate for tipping caused by a large load at the end-effector. 


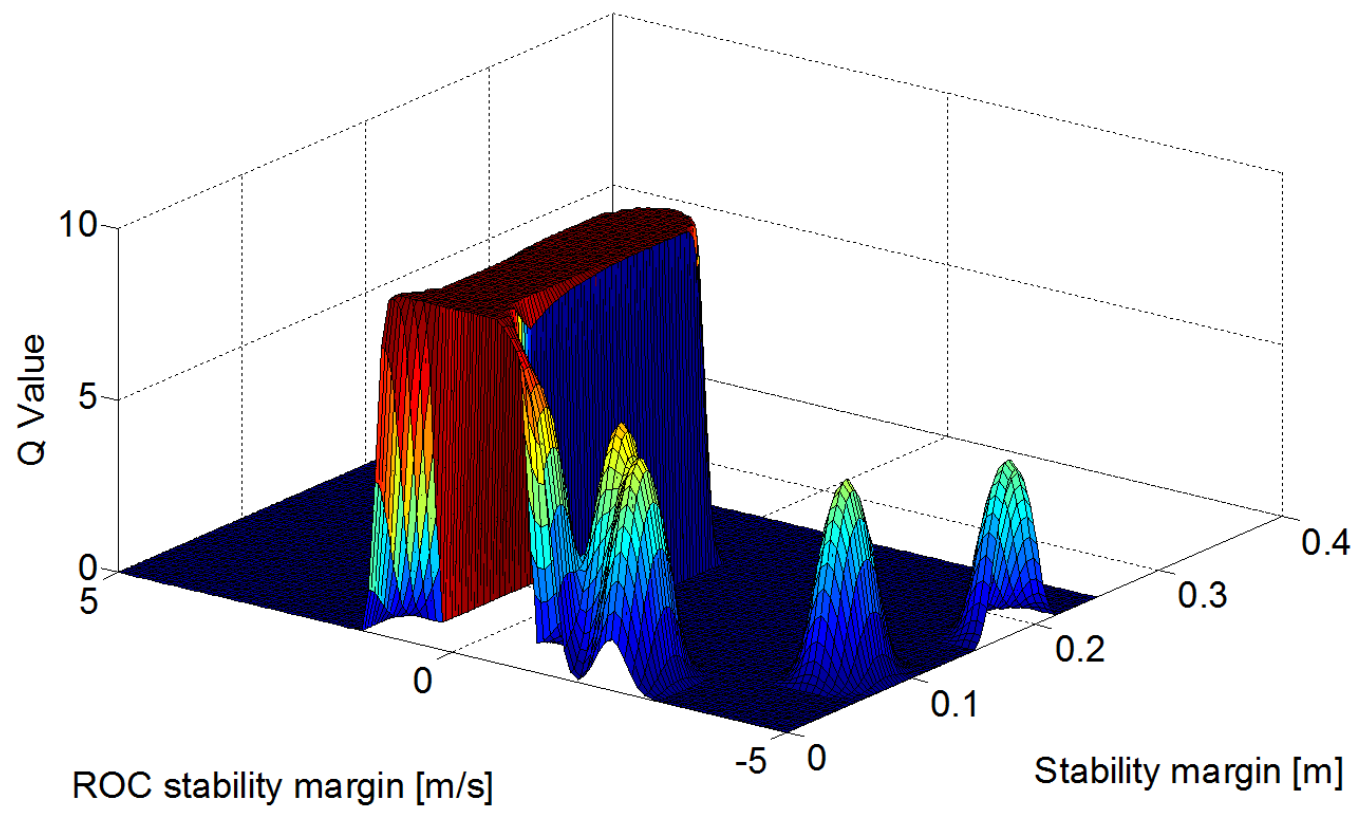

Figure 3.8: Q-function for the behaviour combination preventing tip over, when an $85 \mathrm{~kg}$ load is placed at the end-effector.

the reference behaviour combination of, $\vec{A}=\left[\begin{array}{lll}1 & 1 & 1\end{array}\right]^{T}$, and $\lambda=40^{\circ}$ is shown in Figure 3.10 This figure shows that negative reward is accumulated for, $S_{m}<0.05$. Using the same set of behaviours used in the previous test case, the BBLC is able to find that the behaviour combination of $\vec{A}=\left[\begin{array}{lll}3 & 1 & 3\end{array}\right]^{T}$ results in a balancing strategy that successfully maintains balance on the slope with the motion shown in Figure 3.12. The search for the balancing strategy is exhaustive, meaning all possible behaviour combinations were attempted by the learning algorithm (from $\vec{A}=\left[\begin{array}{lll}1 & 1 & 1\end{array}\right]^{T}$ to $\vec{A}=\left[\begin{array}{lll}3 & 3 & 3\end{array}\right]^{T}$. In the successful strategy, the controller only moves the COM of the robot such that the ZMP remains in the support polygon, while excluding other task-space motions. In other words, when a fall is imminent, the robot ignores other tasks and only maintains balance by moving the COM as the highest priority task. Additionally, in the same region where the reference behaviour accumulated a negative Q-value, the successful strategy accumulates positive reward as show in Figure 3.11. The controller was able to adapt to a new disturbance, using the same behaviour library as used previously to find a new emergent strategy. These results are generalized for similar cases, where the slope manoeuvre varies from the specific learning case initially 


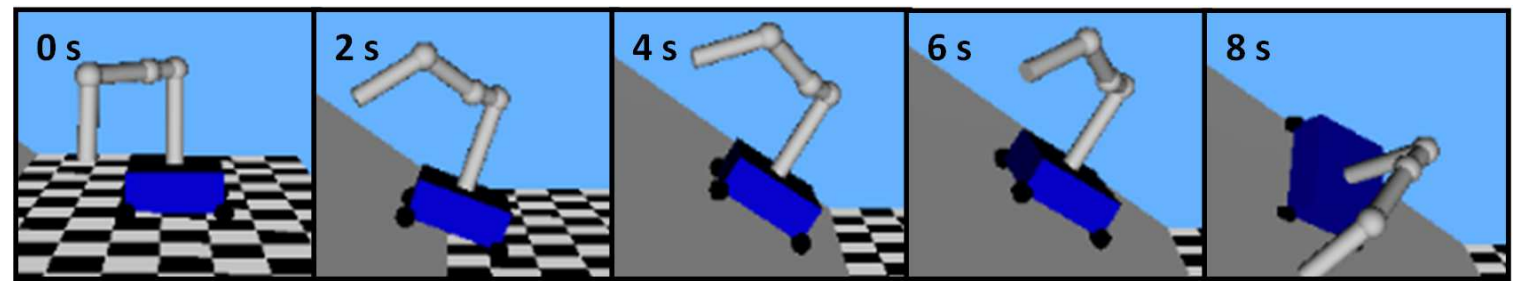

Figure 3.9: Mobile-manipulator tipping over on a $40^{\circ}$ slope.

tested for. In other words, the same strategy was applied for slopes of different angles than of the learnt case.

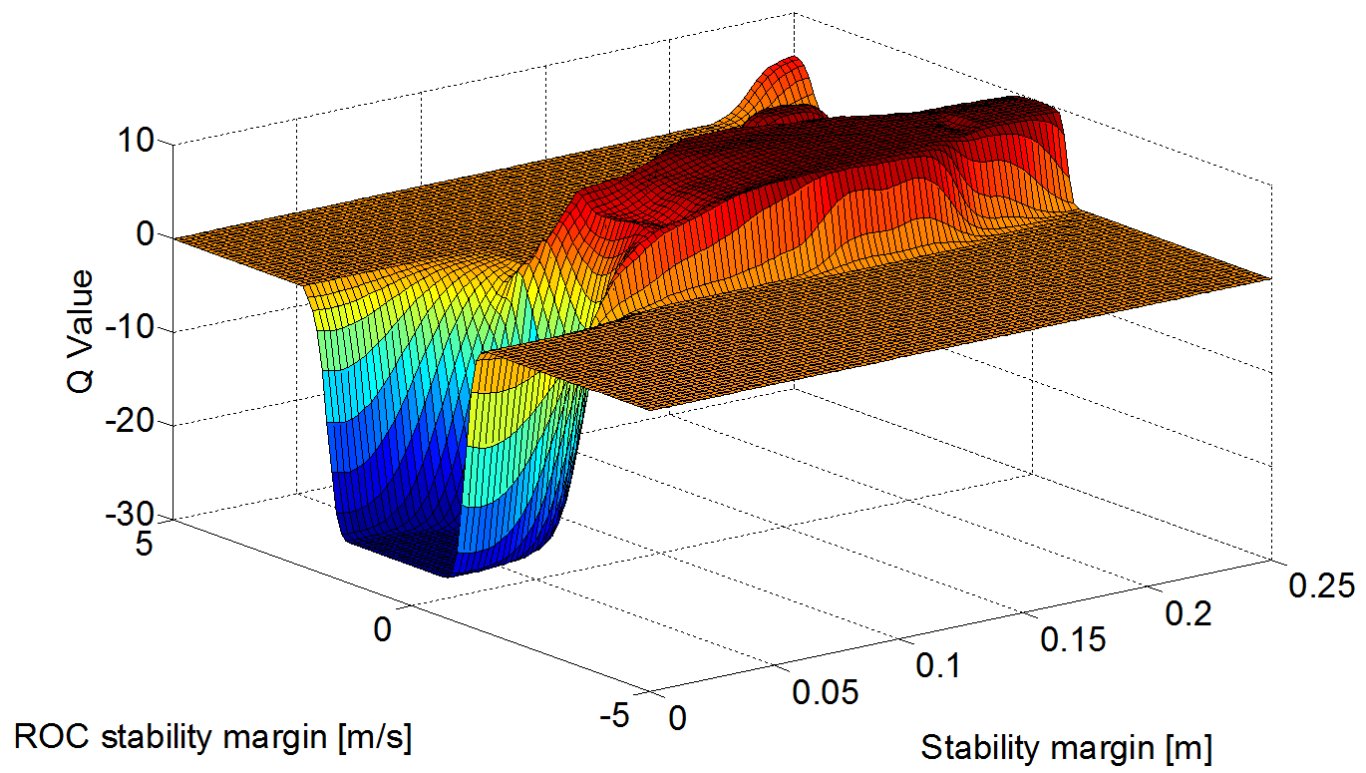

Figure 3.10: Q-function for the behaviour combination used for reference trajectory tracking.

In summary, the results from both experiments with the mobile manipulator show that the BBLC was able to generate an emergent balancing strategy by combining different behaviours for the task-space motions. Hence, the same features found in BBC applied to a single task system were reproduced by extending the BBC architecture to encompass multiple tasks. A more complex composite control emerged from the combination of lower level behaviours, and this emergent control was able to adapt to new environment conditions. 


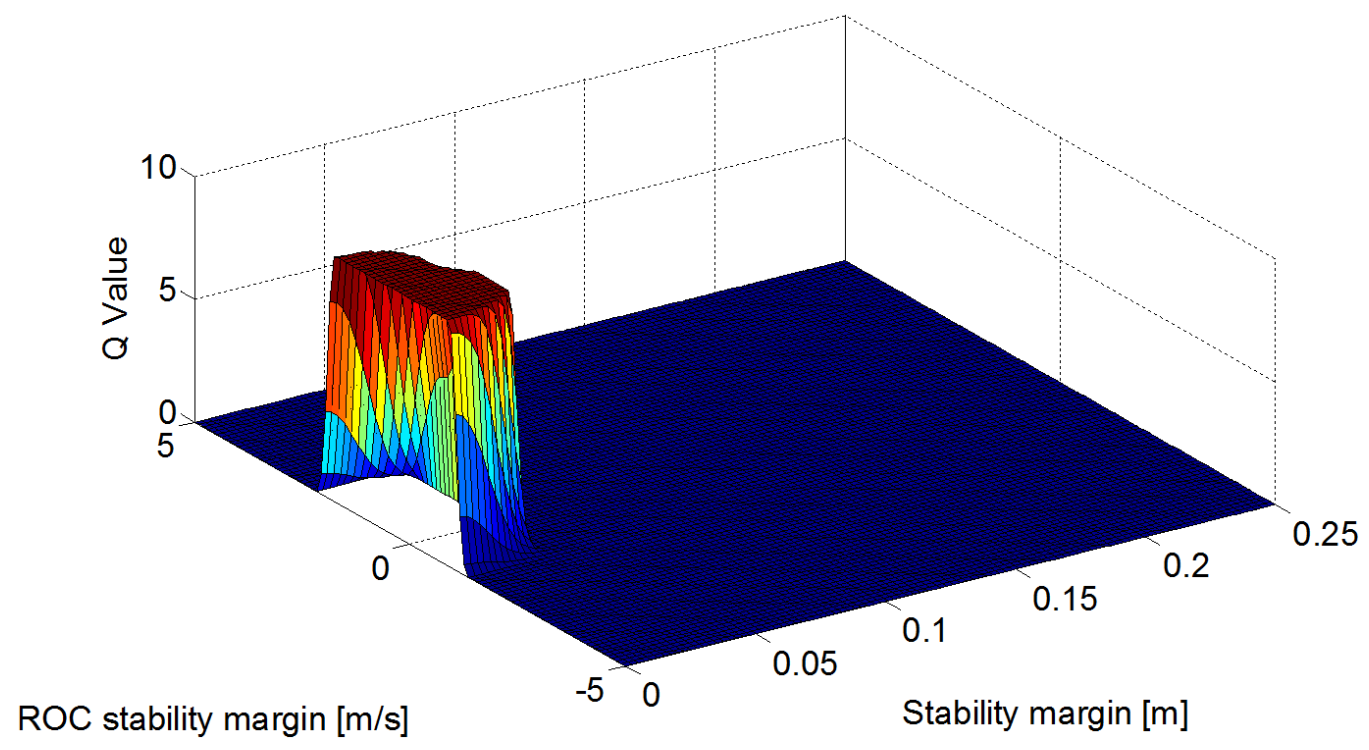

Figure 3.11: Q-function for the behaviour combination resulting in a successful stabilization strategy on sloped surfaces.

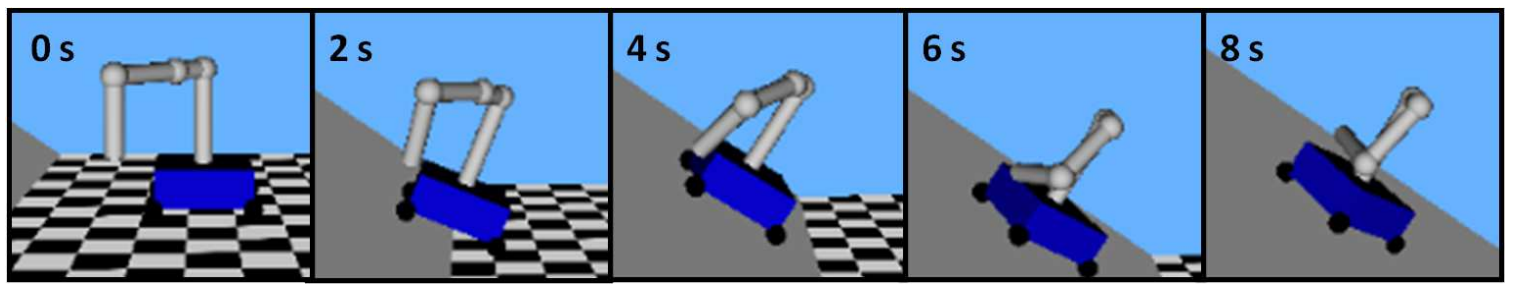

Figure 3.12: Mobile-manipulator recovering on a $40^{\circ}$ slope. 


\subsection{Planar Biped Balance Control}

The BBLC is implemented on a 7 link, planar biped presented in Chapter 2. The objective of the simulation was to determine whether the algorithm was able to find behaviour combinations that can stabilize the robot in the presence of different disturbances. Two different conditions are tested, (1) a push disturbance on flat terrain, and (2) a push disturbance on sloped terrain.

\subsubsection{Task Layer}

As discussed in Chapter 1, the minimal task-space motions required to generate a stable walking gait for a biped robot are: (1) shifting the COM to maintain balance and (2) swinging the non-stance foot to generate a walk. For the BBLC implementations on the planar biped, five task-space motions are defined as follows:

1. Controlling the ankle orientation with respect to the ground $X_{1} \in \mathbb{R}^{2}$.

2. Positioning of the swing leg $X_{2} \in \mathbb{R}^{2}$.

3. Controlling torso posture orientation $X_{3} \in \mathbb{R}^{1}$.

4. Positioning of the COM in the vertical (Y) direction $X_{4} \in \mathbb{R}^{1}$.

5. Positioning of the COM in the horizontal (X) direction $X_{5} \in \mathbb{R}^{1}$.

In addition to the swing foot control and COM based task-space motions, additional taskspace motions have been defined for controlling the ankle orientation and torso posture. These have been defined so that their trajectory can be separately planned, and therefore different behaviours can be created for these task-space motions which may be relevant in compensating for specific disturbances such as a slope. For the biped, the joint space consists of the seven actuated revolute joints as defined in Figure 2.12 


\subsubsection{Behaviour Layer}

A summary of the set of locomotion behaviours for each task-space controller is listed in Table 3.3. The individual behaviours are defined by the designer and can range in complexity from defining a task-space motion to a specific point, to a more complex control method based on a dynamic model of the system. The behaviours presented here are a mix of control strategies that have been developed as balancing behaviours in previous controllers and behaviours that are intuitively defined as potential balancing behaviours. This set of behaviours are an example of a behaviour set that can be used for bipedal balance. A different behaviour set could be implemented depending on the objectives of the designer and the applications being considered.

As with the mobile manipulator example an "exclude behaviour" excludes a task from the task priority coordination in (3.5). The ZMP-based behaviours plan the COM position to maintain the ZMP within the support polygon of the biped. A momentum regulation behaviour-based on the Centroidal Moment Pivot (CMP) criterion [37] plans the COM position to indirectly regulate the total momentum about the COM of the robot. With this set of behaviours for 5 task-space controllers, there are 128 possible different combinations of behaviours that can be sent to the task layer.

\subsubsection{Behaviour Learning Algorithm}

For the biped application the reward function is defined as

$$
R=\left\{\begin{array}{rll}
1 & \text { pelvis height }>0.5 & {[\mathrm{~m}] \text { \& leg is swinging }} \\
0.1 & \text { pelvis height }>0.5 & {[\mathrm{~m}] \& \text { leg is not swinging }} \\
-1 & \text { pelvis height } \leq 0.5 \quad[\mathrm{~m}]
\end{array} .\right.
$$

This reward function gives a negative reward if a fall is detected and a reduced reward if forward motion is stopped. This allows behaviour combinations that allow for forward motion to be selected over those that stop the walking motion. Finally, the Q-function is approximated with a lookup table which is updated in each learning step. The stability 
Table 3.3: Behaviours for planar biped implementation.

Foot ankle orientation control

1-Align ankle orientation with flat floor

2-Adjust ankle orientation for sloped floor

\begin{tabular}{l}
\hline \hline Swing leg trajectory control \\
\hline \hline 1-Swing leg with reference gait parameters \\
2-Exclude leg swing \\
\hline \hline
\end{tabular}

Torso posture orientation control

1-Adjust posture to reference posture position

2-Exclude posture control

COM vertical position control

1-Plan reference COM based on ZMP

2-Plan reference COM based on ZMP with higher gains

3-Plan reference COM based on CMP 37

4-Exclude COM vertical position control

COM sagittal position control

1-Plan reference COM based on ZMP

2-Plan reference COM based on ZMP with smaller stability

margin

3-Plan reference COM based on CMP

4-Exclude COM sagittal position control 


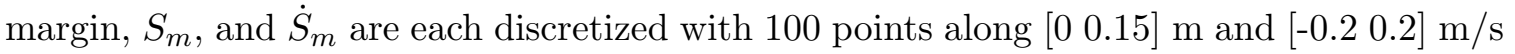
respectively. The slope estimate, $\lambda$, is discretized along [0 10] degrees with 10 points. The values for each cell in the Q-function lookup table are initialized to zero.

\subsubsection{Experiments}

For the planar biped two different experiments are presented. In the first experiment, a push-type disturbance is applied to the torso of the robot. In the second experiment, a push-type disturbance is applied in addition to the robot walking down a slope. As with the mobile manipulator example, the BBLC does not know what kind of disturbance is being applied a priori. The primary objective of these experiments is to examine if the BBLC will be able to identify the unbalanced state of the system and find a new balancing strategy to compensate for the disturbance.

\section{Push Disturbance}

In this experiment the planar biped begins walking on flat level ground with the gait parameters listed in Table 3.4. Once the biped settles into a periodic walking pattern, a 40 Ns (20 N during $2 \mathrm{~s}$ ) impulse is applied to the torso of the robot while the robot is in single stance as shown in Figure 2.12. The impulse force is applied during single stance as this consists of a worst case scenario for maintaining balance. The support polygon during this stance of walking is the smallest, and therefore the robot is less robust to tipping. If

the reference walking behaviour combination, where $\vec{A}=\left[\begin{array}{lllll}1 & 1 & 1 & 1 & 1\end{array}\right]^{T}$, the robot maintains forward walking by using the standard ZMP planning method, and swinging the leg with predefined gait parameters, such as the stride length. Using this action vector results in

Table 3.4: Gait parameters.

\begin{tabular}{lr}
\hline Parameter & Value \\
\hline Step size & $0.1 \mathrm{~m}$ \\
Swing time & $3 \mathrm{~s}$ \\
Double stance time & $3 \mathrm{~s}$ \\
\hline
\end{tabular}


a fall which is reflected in the Q-function, shown in Figure 3.13 . From this function, we see that in the region where $S_{m}>0.06$ and $\dot{S}_{m}>-0.03$, the reference walking behaviour combination generates stable walking and positive reward is given. However, when the disturbance is applied, this causes the stability margin to decrease. Hence, in the range where $S_{m}<0.06$ and $\dot{S}_{m}<-0.03$, the Q-function becomes negative. Using the greedy policy selection algorithm, the learning algorithm finds that with $\vec{A}=\left[\begin{array}{lllll}1 & 2 & 2 & 3 & 3\end{array}\right]^{T}$ the controller is able to stabilize the robot. The resulting motion is shown in Figure 3.14 and the Q-function for the behaviour combination is shown in Figure 3.15. Comparing the resulting Q-function in Figure 3.15 to the one in Figure 3.13, it can be seen that the resulting stabilization strategy accumulates positive reward in the same region where the reference behaviour fell, and accumulated negative reward. This successful behaviour combination results in the robot stopping its swing leg and modifying the COM planning to the angular momentum regulation based strategy for both the sagittal and vertical COM position. Excluding the swing leg motion from the control allows the controller to use all the robot's joints to track the reference COM trajectory more accurately. This result shows that the BBLC algorithm is able to find a combination of behaviours that led to stabilization of the biped to an unknown push-type disturbance. The resulting strategy emerges from the behaviour combination without being planned a priori. The generality of this result was then examined by applying disturbances of varying magnitude from $-25 \mathrm{~N}$ to $25 \mathrm{~N}$. It was found that the new balancing strategy was initiated for these different disturbances without any additional learning. This indicates that one learning case may be sufficient to develop a new balancing strategy for a given type of disturbance, such as a push-type disturbance.

\section{Reinforcement Learning Process}

As described in Section 3.1.3, the objective of the reinforcement learning layer is to find a behaviour combination that will stabilize the robot to a particular disturbance. Learning occurs by exposing the robot to a specific disturbance and iterating through different action

vectors, $\vec{A}$, until one that stabilizes the robot is found. The parameters used for the learning process are listed in Table 3.5. The learning rate parameters $\alpha$ and $\gamma$ were heuristically 


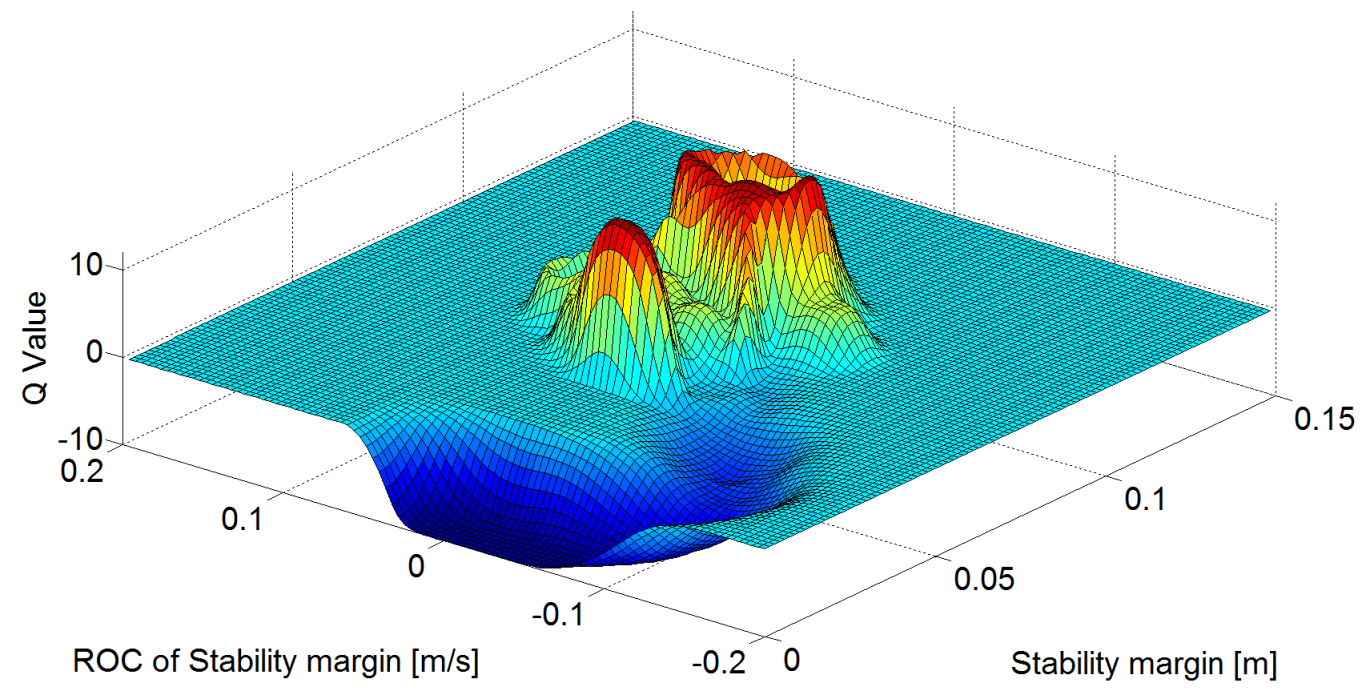

Figure 3.13: Q-function for the behaviour combination used for stable walking on flat terrain. Negative Q-values are accumulated when this behaviour combination is unable to compensate for push-type disturbances.

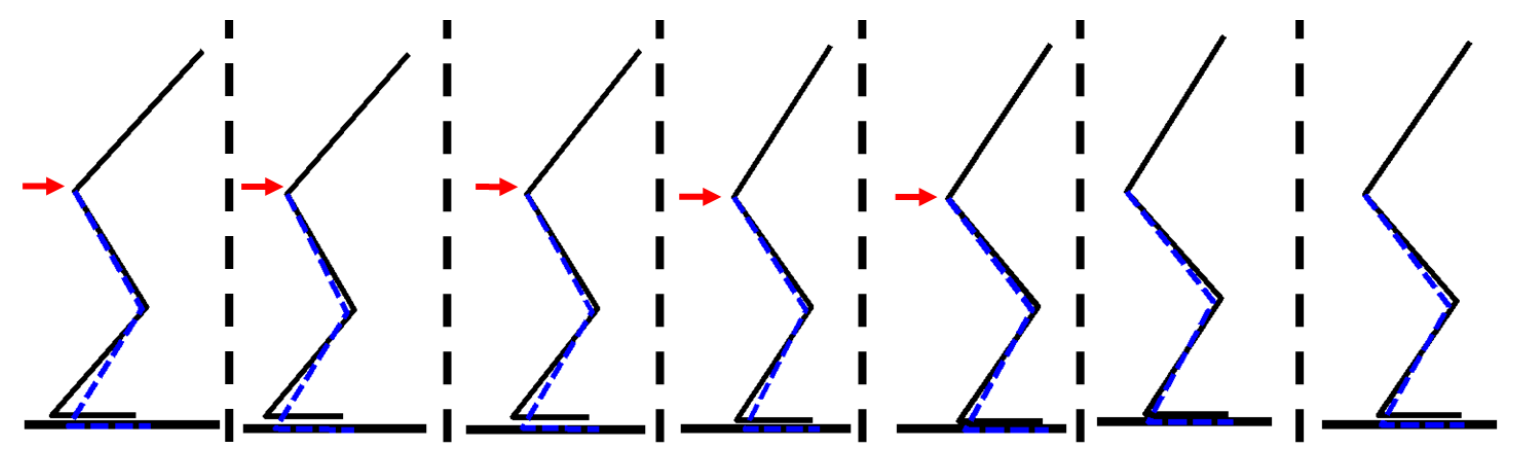

Figure 3.14: Biped motion after push disturbance on a flat terrain. In this motion the biped shifts the COM backwards to counter the effect of the disturbance. 


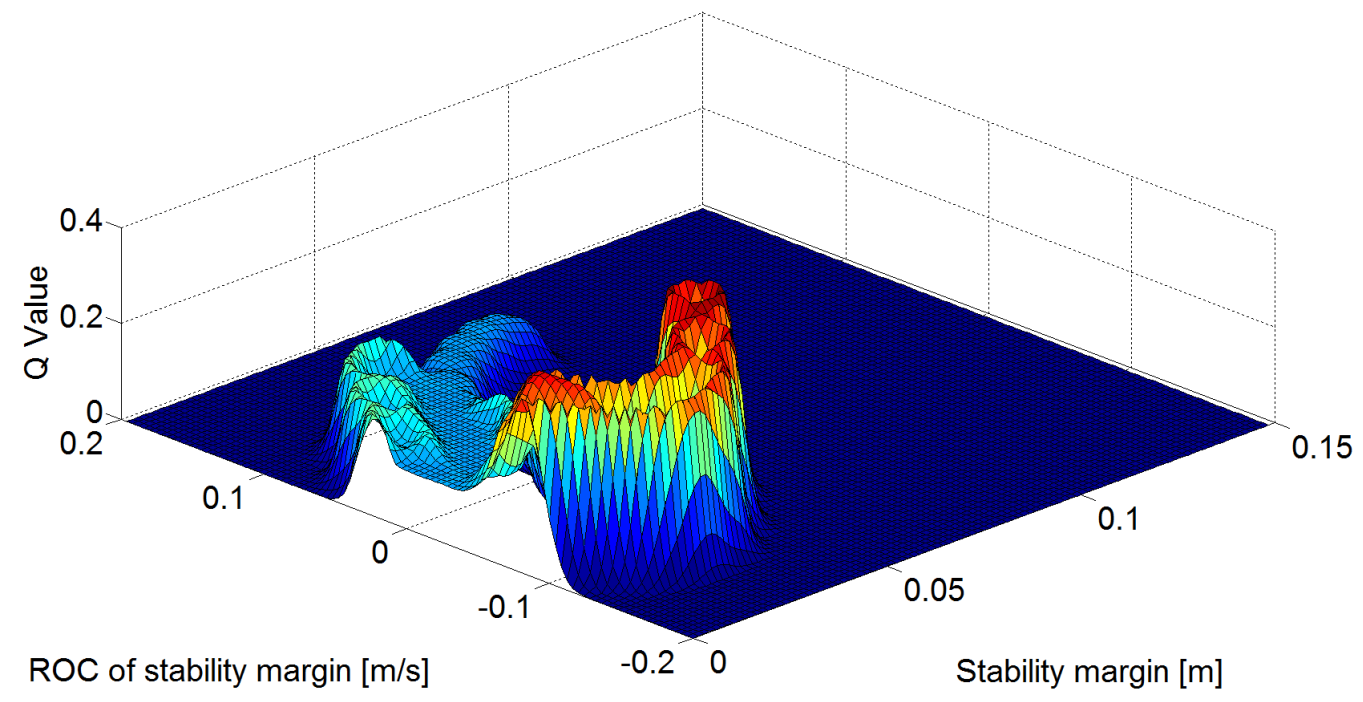

Figure 3.15: Q-function for a behaviour combination capable of stabilizing the robot to push-type disturbances.

selected to be $\approx 1$ to allow for faster learning. With both these parameters $\approx 1$, the previous value for $Q(\bar{s}, \vec{A})$ is discarded and replaced by a newly learnt value. The eligibility trace parameter $\lambda$ was tuned heuristically by alternating between a scenario where a push was applied versus one where no push is being applied. If the balancing strategy was applied when no disturbance was present, this indicated that the Q-learning algorithm had learnt to activate a balancing strategy for states which were balanced and therefore could continue using $\vec{A}=\left[\begin{array}{llll}1 & 1 & 1 & 1\end{array}\right]^{T}$. The parameters were found to work well where an eligibility trace memory of 30 learning steps (consisting of a $1.5 \mathrm{~s}$ memory) and $\lambda=0.9$. This resulted in the Q-learning algorithm associating negative reward only with states which resulted in an unbalanced condition.

The initial default action vector in the push disturbance case is $\vec{A}=\left[\begin{array}{lllll}1 & 1 & 1 & 1 & 1\end{array}\right]^{T}$, which does not result in a balanced system when the robot is pushed. This first simulation iteration corresponds to the first episode in Figure 3.16. The second episode corresponds to applying an action vector $\vec{A}=\left[\begin{array}{lllll}1 & 1 & 1 & 1 & 2\end{array}\right]^{T}$. This iteration continues until the action vector $\vec{A}=\left[\begin{array}{lllll}1 & 2 & 2 & 3 & 3\end{array}\right]^{T}$ results in a balanced system, resulting in a higher cumulative reward, as shown in Figure 3.16. At this point learning is stopped given that a successful, strategy has been found. However, an exhaustive search could also be performed, where 
the learning algorithm is forced to iterate through all possible action vectors to determine if other successful balancing strategies exist. Given the discrete action space, the resulting learning curve does not show a gradual improvement of cumulative reward; rather, a discrete jump in cumulative reward occurs when a successful behaviour combination is found, as shown in Figure 3.16, Other variations in the learning curve occur when certain action vectors result in behaviours that will keep the robot balanced longer, but will not prevent a fall. The time required to find a successful behaviour combination, if one exists, will depend on the size of the action space. However, a successful action vector may be found after one episode. Simulation time for this experiment was 20 seconds and simulations were run on a $2.66 \mathrm{GHz}$ processor with $4 \mathrm{~GB}$ of RAM. The resulting learning time and simulation parameters are listed in Table 3.6 .

\section{Slope Combined with Push Disturbance}

The objective of the second experiment is to examine whether the algorithm can compensate for a different kind of disturbance with the same set of locomotion behaviours. The robot is first exposed to a sloped terrain. As expected, the robot must apply the Adjust ankle orientation for sloped floor behaviour for the ankle control task-space controller. The learning algorithm successfully identifies that the behaviour combination capable of walking on sloped terrain is $\vec{A}=\left[\begin{array}{lllll}2 & 1 & 1 & 2 & 1\end{array}\right]^{T}$. Similarly to the flat terrain test, if a push-type disturbance (40 Ns impulse) is applied to the robot, using this reference behaviour combination fails to stabilize the robot with the resulting Q-function shown

Table 3.5: Reinforcement learning algorithm parameters.

\begin{tabular}{lr}
\hline Parameter & Value \\
\hline$\gamma$ & 0.99 \\
$\alpha$ & 0.99 \\
$\lambda$ & 0.9 \\
Learning time step & $0.05 \mathrm{~s}$ \\
Eligibility trace memory & 30 learning steps \\
\hline
\end{tabular}




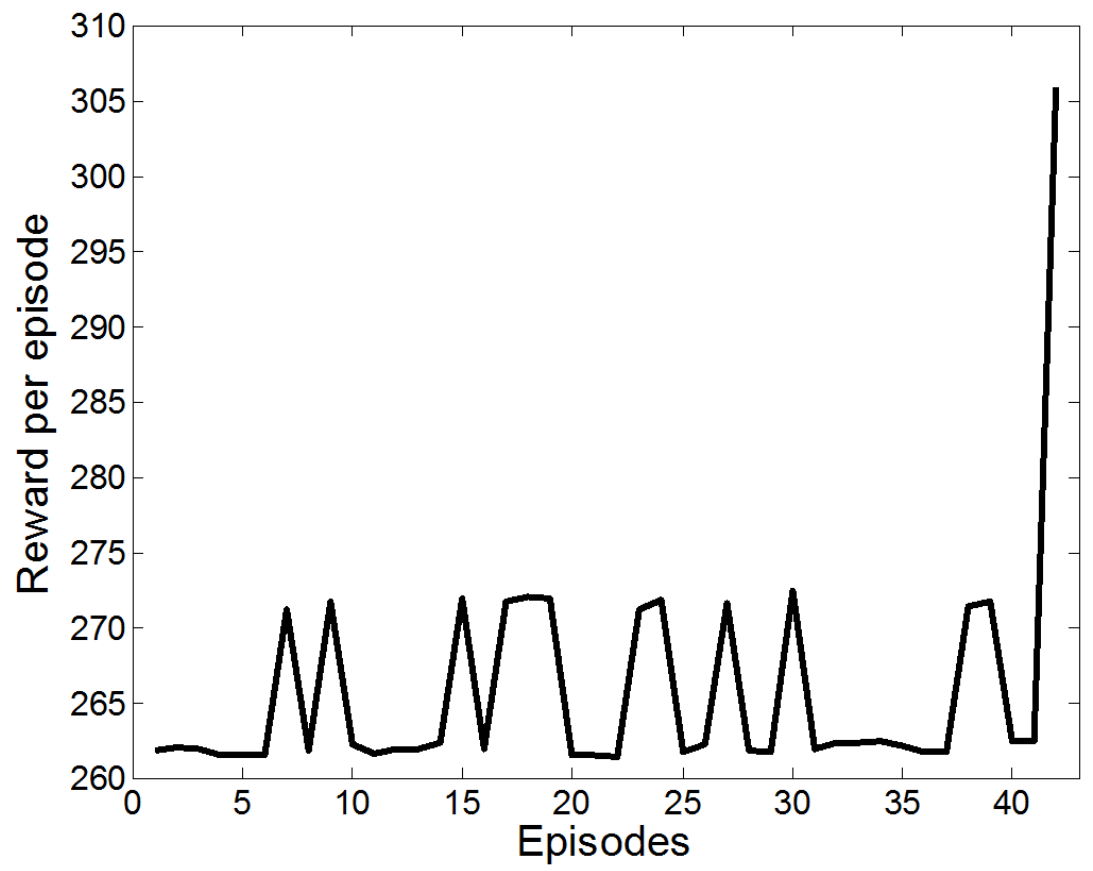

Figure 3.16: Learning curve for biped robot push test on flat terrain. A successful behaviour combination is found after 42 episodes.

in Figure 3.17, where $\lambda$ is fixed at $10^{\circ}$. The algorithm finds that behaviour combination $\vec{A}=\left[\begin{array}{lllll}2 & 2 & 2 & 2 & 3\end{array}\right]^{T}$ stabilizes the robot with the resulting Q-function shown in Figure 3.18 and motion shown in Figure 3.19. This behaviour combination results in a similar stop and regulate angular momentum strategy generated in the 'push disturbance on flat terrain' case. From this experiment, the results show that by using the same set of behaviours, some of which were also used in the flat terrain push experiment, the controller was able to generate a strategy that stabilizes the robot.

Table 3.6: Reinforcement learning performance for bipedal robot simulation.

Computation time per episode with learning $101.1 \mathrm{~s}$

Computation time per episode without learning $\quad 62.2 \mathrm{~s}$

Dynamic simulation fixed time step $\quad 0.0001 \mathrm{~s}$ 


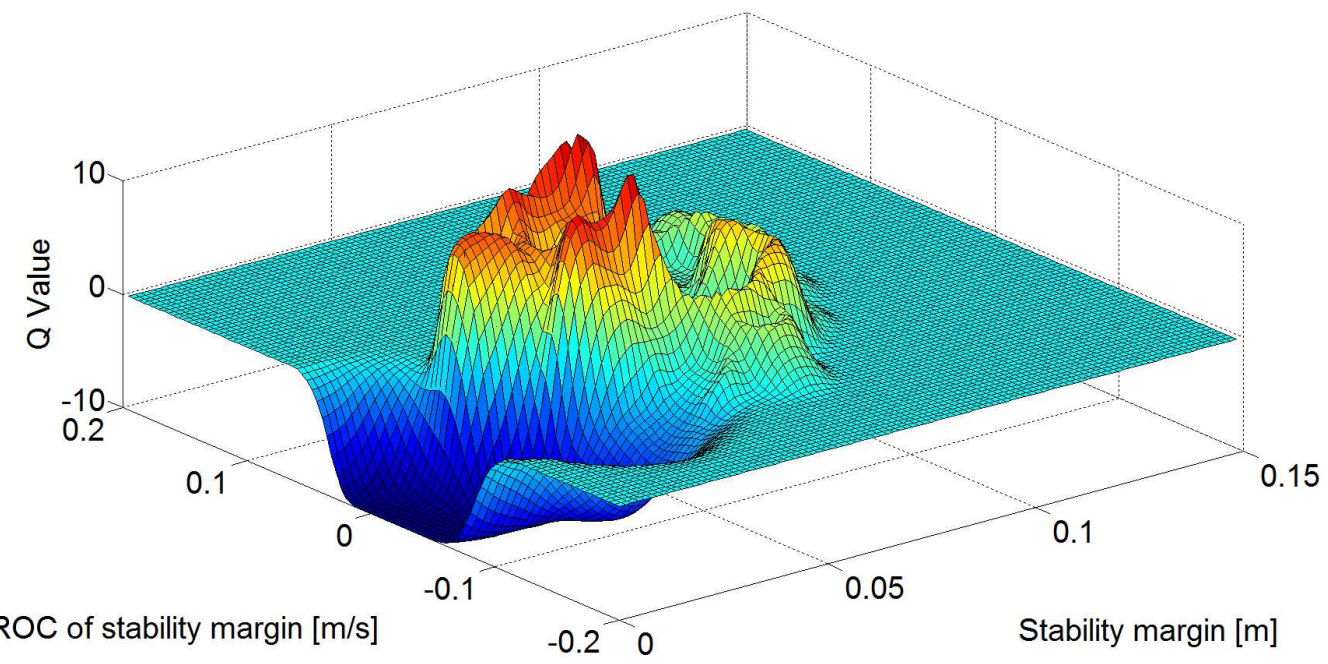

Figure 3.17: Q-function for reference walking behaviour combination on sloped terrain.

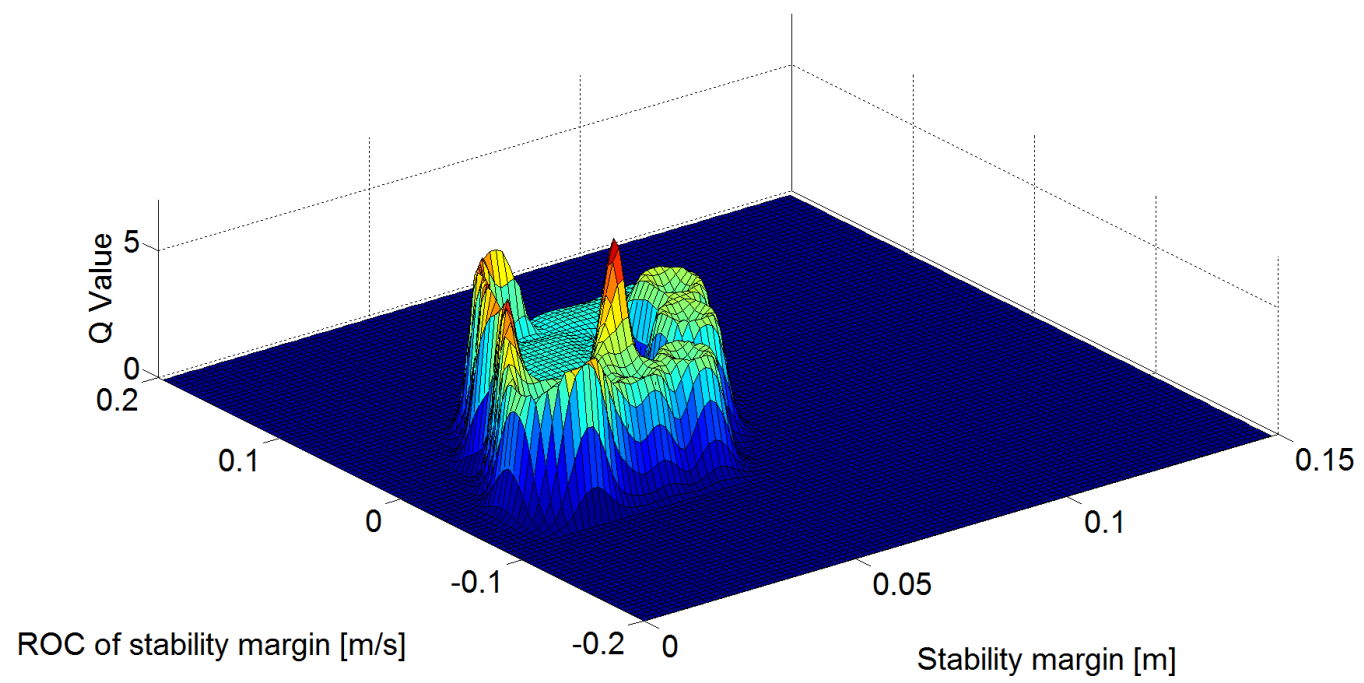

Figure 3.18: Q-function for the behaviour combination capable of stabilizing the robot when a push-type disturbance is applied on a slope. 


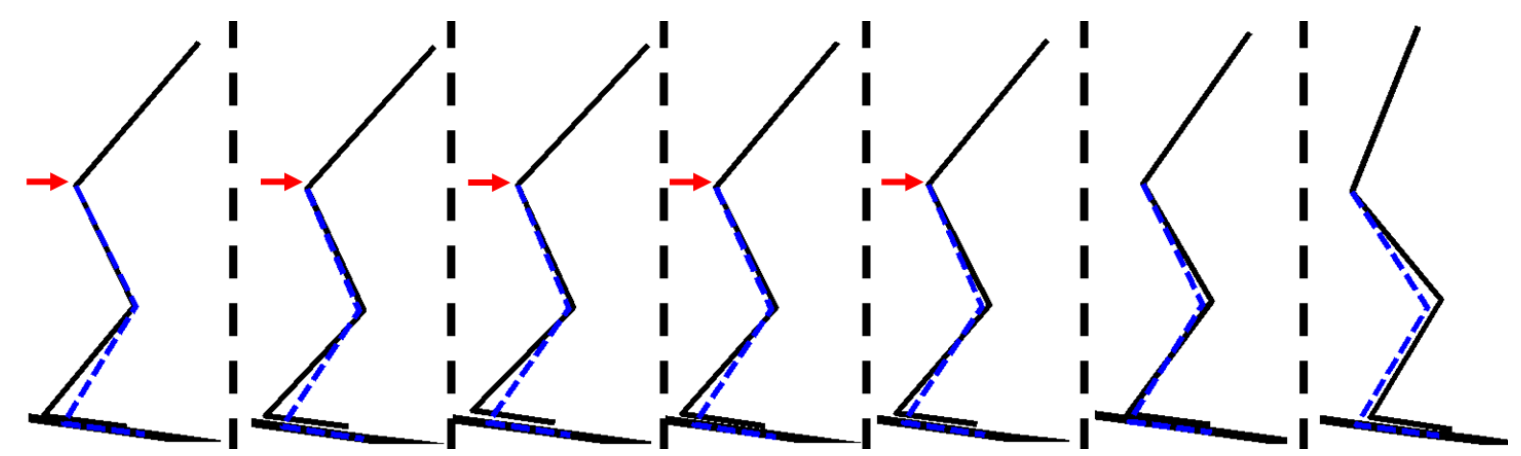

Figure 3.19: Biped motion after a push disturbance on a $10^{\circ}$ slope.

In summary, the biped example provided a more complex system with additional taskspace motions and behaviours for the BBLC to coordinate. Nevertheless, as with the mobile manipulator example, the BBLC algorithm was able to identify behaviour combinations that maintained balance when new, unknown disturbances were applied. These behaviour combinations resulted in new, more complex balancing strategies that emerged without a priori knowledge of the type of disturbance being applied or of the time of application. These results suggest that using the $\mathrm{BBC}$ approach in robotic locomotion can generate emergent balancing strategies for a wide range of unknown disturbances (such as terrain variances or external pushes), by using a fixed library of behaviours for the task-space motions.

\subsection{Chapter Summary}

This chapter introduced a general architecture for the BBLC along with two demonstrative examples. There were three research questions that were examined in this chapter. The first was if the BBC architecture could be augmented to complex locomotion systems with multiple task-space objectives and redundancy. The BBLC architecture presented showed that this could be achieved by applying a multi-layered control architecture, consisting of:

- A Task Layer that coordinates the multiple task-space motions in the system and defines final joint-space trajectories.

- A Behaviour Layer consisting of different task-space trajectory planning behaviours 
for each task-space motion.

- A Reinforcement Learning Layer, which identifies which combinations of behaviours can result in new balancing strategies.

A second core research question was whether or not this novel control architecture could generate new balancing strategies without knowledge of the disturbance. To demonstrate this ability, two examples, a planar biped robot and mobile manipulator, were tested in simulation. In both cases results showed that the BBLC was able to find balancing strategies to unknown disturbances that were able to maintain the robot balanced, for a set of simpler task-space behaviours. The results confirm that the core hypothesis behind the BBLC is valid - balancing strategies can emerge from a simpler set of balancing behaviours.

The last question examined in this chapter was if the BBLC architecture was generalizable to redundant systems in robotic locomotion. To test this, the BBLC was applied to two different systems, a mobile manipulator and a biped robot. In both cases, the controller successfully found new balancing strategies, despite the different kinematic and dynamic properties of each system. Overall, this result suggests that the BBLC could be applied to redundant robotic systems with multiple task-space motions, where maintaining balance is the main control objective. 


\section{Chapter 4}

\section{BBLC Implementation on ABL-BI: Simulation}

The focus of this chapter is to present how the BBLC is implemented on the ABL-BI robot followed by simulation experiments. This consists in implementing the BBLC on a 3D biped, as opposed to the 2D planar example examined in Chapter 3. The additional challenges with moving to a 3D platform include consideration for lateral stability, increased number of DOFs and planar ground contacts (as opposed to two point linear contacts in 2D). The description of the BBLC implementation on ABL-BI will include a detailed description of the individual behaviours that were created for the ABL-BI robot in Section 4.1. The behaviours presented will be the same for both the simulation model presented in Chapter 2, and the controller implemented on the experimental platform. The simulation focused on exposing the controller to three different disturbances: a push, a step under one foot and a slope.

The primary research aim is to determine if the BBLC can be successfully implemented

on the ABL-BI platform with the same results as presented in Chapter 3, i.e., will the BBLC be able to generate emergent balancing strategies for unknown disturbances in the 3D bipedal balance case. The second key research objective in this chapter is to perform a more in depth analysis of the emergent balancing strategies. The generalizability of the emergent strategies is evaluated by examining if these newly learnt strategies will be activated by a disturbance of a similar nature, but different quantitively than the learnt case. Additionally, the behaviour of the BBLC to transient disturbances is examined. The 
results for each of the disturbance cases are presented in Section 4.2 .

\subsection{BBLC for ABL-BI}

As described in Chapter 3, the BBLC is divided primarily into three separate layers: the Task Layer, the Behaviour Layer and the Learning Layer. Although the mathematical structure for each layer is maintained for the complete 3D implementation for ABL-BI, this section will give the specific details for each layer in Sections 4.1.1 to 4.1.3. The individual behaviours for each task-space motion are described in Section 4.1.2.

\subsubsection{Task Layer}

As discussed in Chapters 2 and 3, the principle task-space motions required for walking are shifting the COM position and foot swing motions. Additionally, other task-space motions such as ankle and torso motions can be defined to plan separate behaviours for these tasks which may be relevant for certain disturbances. For the 3D biped, there is a total of six separate task motions:

1. Ankle motion - landing angle of the foot $X_{1} \in \mathbb{R}^{2}$

2. Foot swing motion $X_{2} \in \mathbb{R}^{3}$.

3. X COM motion $X_{3} \in \mathbb{R}^{1}$.

4. Y COM motion $X_{4} \in \mathbb{R}^{1}$.

5. Z COM motion $X_{5} \in \mathbb{R}^{1}$.

6. Torso motion $X_{6} \in \mathbb{R}^{1}$.

The Ankle motion consists of defining the landing angle of the foot at the end of the swing. The Foot swing motion consists of defining the trajectory of the swing foot while 
the robot is walking. For this task, the foot trajectory is defined in Cartesian space and only the joints in the swing leg are included in the task-space Jacobian. All three $\boldsymbol{C O M}$ motion task-space trajectories consist of defining a trajectory for the COM in Cartesian space. With each axis consisting of an individual task-space motion, different behaviours can be individually applied to each axis separately. For each of these motions, the Jacobian makes use of all joints, and is derived from the forward kinematics of the COM position. The Torso motion consists of defining the trajectory of the torso angle independently. As described in Chapter 2, the torso has the ability to rotate about the $\mathrm{Z}$ axis, therefore the torso angle is a function of joint 13 only (for joint definitions please refer to Chapter 2).

\subsubsection{Behaviour Layer}

As discussed in Chapter 3, the Behaviour Layer defines different methods of planning reference trajectories for the task-space motions in the Task Layer. This section will provide an overview of each behaviour for the six task-space motions defined in Section 4.1.1. Table 4.1 provides a summary of all the behaviours, and the corresponding numbering scheme that is used for the action vector $\vec{A}$.

\section{Ankle motion behaviours}

There are three behaviours that are defined for the ankle motion. As for all task-space motions, one of these is an exclude behaviour, where the ankle motion is not controlled in the task-coordination algorithm in Equation 3.5. The other two consist of controlling the foot angle based on walking on flat or sloped terrain.

For walking on flat terrain the reference foot pitch angle $\alpha_{r e f}$ is defined to simply be $\alpha_{r e f}=0^{\circ}$, which results in the foot landing flat on the floor. For sloped walking an estimate of the slope is established from the foot contact kinematics as shown in Figure 4.1 
Table 4.1: Summary of behaviours for each task-space motion.

\begin{tabular}{l}
\hline Ankle angle \\
\hline \hline 1- Flat terrain walking \\
2- Sloped terrain walking \\
3- Exclude
\end{tabular}

Swing leg motion

1- Walking with $0.1 \mathrm{~m}$ stride

2- Walking with $0.12 \mathrm{~m}$ stride

3- Exclude

\section{COM motion}

1- ZMP based planning

2- CMP based planning

3- CMP based planning with reference CMP set to middle of support polygon

4- Exclude

\section{Y COM motion}

1- Track COM height of $0.46 \mathrm{~m}$

2- Track COM height of $0.44 \mathrm{~m}$

3- Exclude

\section{Z COM motion}

1- ZMP based planning

2- CMP based planning

3- CMP based planning with reference CMP set to middle of support polygon

4- Exclude

\section{Torso motion}

1- Move torso with weight shifts to left and right foot

2- Move torso to far right

3- Move torso to middle

4- Move torso to far left

5- Exclude 


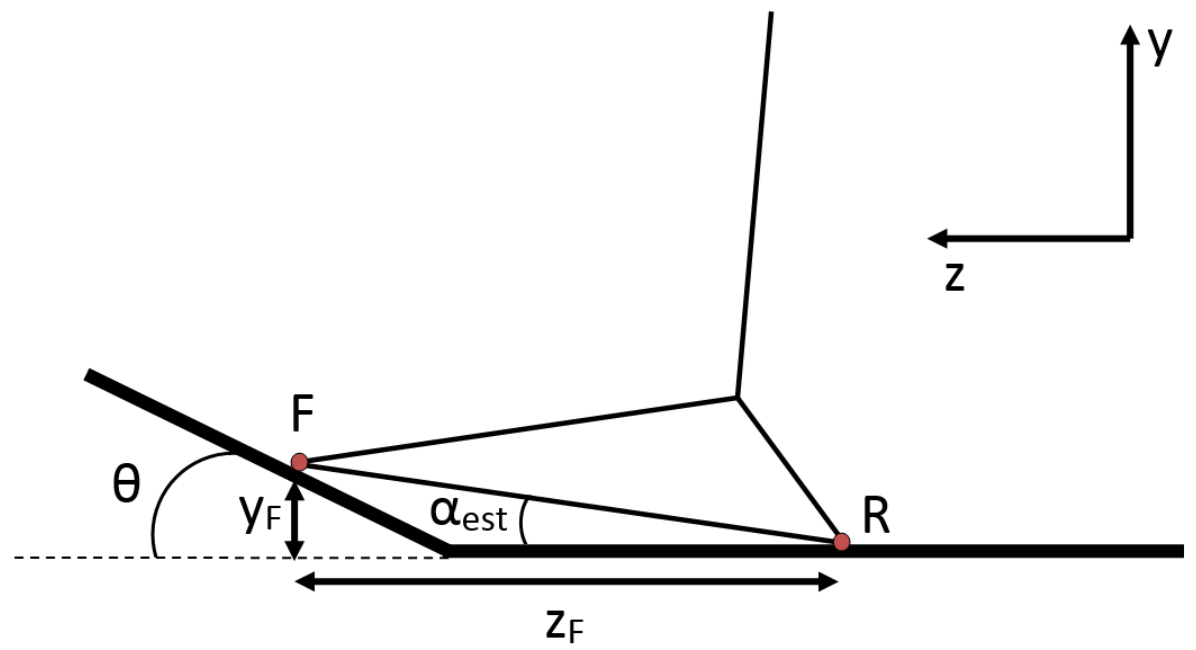

Figure 4.1: The method of estimating a slope angle is illustrated. The height difference at landing between the front and rear of the foot are used to estimate the ground slope angle $\theta$.

and calculated as

$$
\alpha_{e s t}=\arctan \left(\frac{y_{F}-y_{R}}{z_{F}}\right),
$$

where, $\alpha_{e s t}$ is the slope estimate, $y_{F}$ and $y_{R}$ are the height of the front and rear of the foot respectively and $z_{F}$ and $z_{R}$ are the position of the front and rear of the foot along the $\mathrm{Z}$ axis. During walking, the estimate is continually updated and the landing angle of the swing foot is set to the estimated slope angle.

\section{Swing Leg Behaviours}

There are three swing leg behaviours, including an exclusion behaviour. The other two behaviours define two different stride lengths. The first behaviour defines a stride length of $0.1 \mathrm{~m}$, while the second defines a stride length of $0.12 \mathrm{~m}$. The standard stride is a stride length which can easily be tracked by ABL-BI within its workspace. A larger stride results in a larger support polygon during double stance, which may result in greater stability. Reference trajectories for the $\mathrm{Y}$ axis are defined using cubic splines, with a constant peak step height of $0.03 \mathrm{~m}$. This swing height was found to provide sufficient clearance from the 
(a)

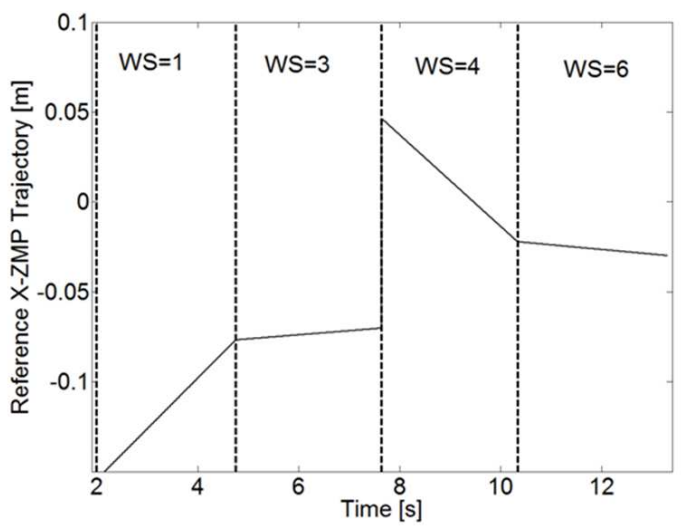

(b)

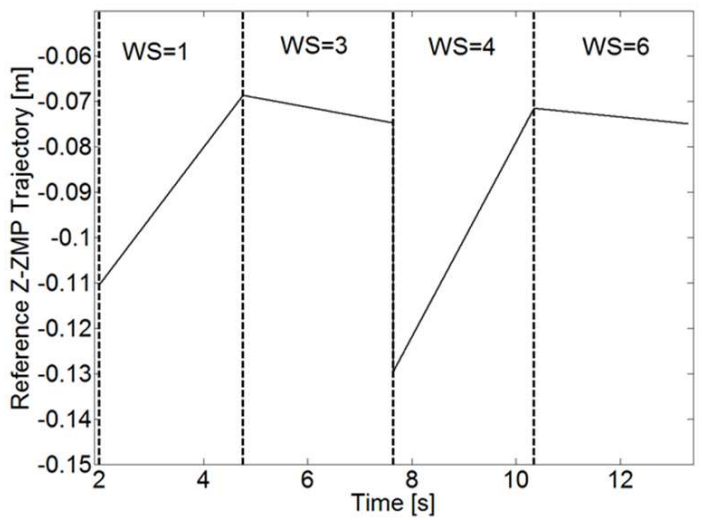

Figure 4.2: Reference ZMP or CMP trajectories for the $\mathrm{X}$ and $\mathrm{Z}$ axes for a cycle of walking. The figure is divided between different walking stances as defined in Chapter 2 (WS=1 double stance left foot, $\mathrm{WS}=3$ single stance left foot, $\mathrm{WS}=4$ double stance right foot, $\mathrm{WS}=6$ single stance right foot). Note a step occurs between $\mathrm{WS}=3$ and $\mathrm{WS}=4$ due to a change in reference frames.

ground during walking, although higher or lower swing heights could be used as well.

\section{COM Motion Behaviours for $\mathrm{X}$ and $\mathrm{Z}$ axis}

The behaviours for the $\mathrm{X}$ and $\mathrm{Z}$ axes are similar and differ only in the magnitude of the planned trajectories. There are a total of four different behaviours:

1. ZMP-based COM planning.

2. CMP-based COM planning.

3. CMP-based COM planning with conservative stability margin.

4. Exclude.

The ZMP-based behaviour consists of planning the motion of the COM according to the ZMP criterion. The reference ZMP is planned to follow the robot's walking pattern as a function of the stride length and step time, and is forced to lie within the support polygon to ensure balance. The reference ZMP motion is then interpolated using splines to generate 
a continuous trajectory as shown in Figure 4.2. The trajectory of the COM for each axis is then calculated as

$$
\begin{aligned}
& x_{r C O M}=x_{r Z M P}+\frac{\ddot{x}_{C O M} y_{C O M}}{g}, \\
& z_{r C O M}=z_{r Z M P}+\frac{\ddot{z}_{C O M} y_{C O M}}{g},
\end{aligned}
$$

where $x_{r C O M}, z_{r C O M}$ are the reference $\mathrm{COM}$ trajectories, $x_{r Z M P}, z_{r Z M P}$ are the reference ZMP trajectories, $\ddot{x}_{C O M}$, $\ddot{z}_{C O M}$ are the COM accelerations, $y_{C O M}$ is the height of the $\mathrm{COM}$, and $g$ is the acceleration due to gravity. If the reference COM motion defined in Equations 4.2 and (4.3) is tracked, then the ZMP will follow the reference ZMP trajectory. Tracking the reference ZMP motion will keep the robot balanced, as long as significant disturbances are not applied.

The CMP-based behaviours are similar to the ZMP based planning, but rather than using the ZMP criterion, the CMP criterion is used, which results in different method of defining the reference COM motion. As with the ZMP, a reference CMP trajectory is defined to lie within the support polygon as a function of the gait parameters. The reference COM is then calculated as

$$
\begin{aligned}
& x_{r C O M}=x_{r C M P}+\frac{F_{x} y_{C O M}}{F_{y}}, \\
& z_{r C O M}=z_{r C M P}+\frac{F_{z} y_{C O M}}{F_{y}},
\end{aligned}
$$

where, $x_{r C M P}, z_{r C M P}$ are the reference CMP trajectories and $F_{x}, F_{y}, F_{z}$ are the ground reaction forces.

For the third behaviour, the reference trajectory is substituted with a reference CMP positioned in the middle of the support polygon. Using this CMP-based control method alone was investigated in further detail by the author in 37. It was shown that 
planning the COM motion with this method resulted in greater robustness to push-type disturbances, compared to using a ZMP planning method in the first behaviour.

\section{COM Motion Behaviours for Y Axis}

For the Y Axis motion, there are two different trajectories that are defined beyond the exclude behaviour. The first is to hold the Y COM at a constant height of $0.46 \mathrm{~m}$, while the second is to move the COM to a lower height of $0.44 \mathrm{~m}$.

\section{Torso Behaviours}

The torso roll behaviours consist in defining the torso roll angle. The first behaviour consists of rolling the torso from right to left following the weight shift of the walking motion. The other behaviours consist of defining a fixed position for the torso: far right $\left(23^{\circ}\right)$, far left $\left(-23^{\circ}\right)$ or middle $\left(0^{\circ}\right)$.

\subsubsection{Reinforcement Learning Layer}

The reinforcement layer for the ABL-BI implementation consists in defining reduced statespace that can be used to identify the disturbances that the robot is exposed to. As presented in Chapter 3, this reduced state-space $\bar{s}$ can be composed of stability relevant parameters such as the stability margin to reduce the dimensionality of the lookup table. The variables that are considered in this reduced state-space are:

- The stability margin $S_{m}$.

- $\mathrm{X}$ axis GRF $F_{x}$.

- $\mathrm{Z}$ axis $\mathrm{GRF} F_{z}$.

- Walking state $W S$. 
- Slope estimate $\alpha_{e s t}$ as defined in 4.1.

Hence, $\bar{s}=\left[\begin{array}{lllll}S_{m} & F_{x} & F_{z} W S & \alpha_{e s t}\end{array}\right]^{T} \in R^{5}$. As listed in Table 4.2 , each state is discretized with only 10 points for each state except for the walking state which is discretized with 6 points - for the 6 different walking states defined in Chapter 2. There were two reasons to use this coarser discretization compared to the one presented for the examples in Chapter 3. First, from the preliminary implementation of the BBLC in the planar biped example, the results showed that greater resolution of the state-space did not result in improved identification of the applied disturbance. Second, a coarser discretization allowed for smaller computational resources required for learning. With the current discretization there is a total of 60,000 possible state combinations. This corresponds to approximately $0.96 \mathrm{MB}$ of memory required to store a complete lookup table. If each state were discretized with 100 points, this would increase to $6.0 * 10^{8}$ points and $9.6 \mathrm{~GB}$ of memory, which is not feasible for existing embedded controllers. Note that defining the reduced state-space consists of a priori planning - if the robot is expected to walk on slopes, then the designer would include a terrain slope measurement in this reduced state-space to characterize the terrain. Although this is the case, we will see in the examples shown in Section 4.2.3, that the reduced state-space will be able to identify disturbances which are not considered a priori as well.

Reinforcement learning is achieved by applying the Q-learning algorithm defined in Equation (3.7). The learning parameters that were used are listed in Table 4.3. These

Table 4.2: Discretization of the reduced state-space for the Q-value lookup table.

\begin{tabular}{|l|c|r|}
\hline Variable & Range & Discretization \\
\hline Stability Margin & 0 to $0.1[\mathrm{~m}]$ & 10 \\
X GRF & -20 to $20[\mathrm{~N}]$ & 10 \\
Z GRF & -30 to $30[\mathrm{~N}]$ & 10 \\
Walking State & 1 to 6 & 6 \\
Slope Estimate & -5 to $5[\mathrm{deg}]$. & 10 \\
\hline
\end{tabular}


parameters were primarily selected such that, at the beginning, new Q-values would be weighted heavily compared to those already learnt. This is done by choosing $\gamma \approx 1$ and $\alpha \approx 1$. Hence, previously learnt Q-values are discarded in favour of new ones. The eligibility trace memory was set to 30 time steps, which corresponds to a learning memory length of $1.5 \mathrm{~s}$. This was selected as a result of simulation experiments, where it was found that the time between the application of a disturbance and a fall occurring is between $1.5 \mathrm{~s}$ to 2.0 s. Thus, qualitatively, a memory length of $1.5 \mathrm{~s}$ corresponds to a relevant memory of states that are associated with a fall occurring, without encroaching on states which did not cause a fall. Finally, the reward function is defined as

$$
R \equiv\left\{\begin{array}{rl}
1 & \text { Pelvis Height } \geq 0.5[\mathrm{~m}] \\
-1 & \text { Pelvis Height }<0.5[\mathrm{~m}]
\end{array} .\right.
$$

With this definition, positive reward is accumulated when the pelvis remains above 0.5 $\mathrm{m}$ and negative reward is accumulated when the pelvis falls below $0.5 \mathrm{~m}$, which is defined as a fall.

\subsection{Simulation Results}

There were several motivating factors to simulate the BBLC on the 3D dynamic simulation described in Chapter 2. The first was to develop and test the controller in simulation before testing on the experimental platform. The second reason was to perform the learning

Table 4.3: Values of learning parameters used in the reinforcement learning algorithm.

\begin{tabular}{|l|c|}
\hline Parameter & Values \\
\hline$\alpha$ & 0.9 \\
$\gamma$ & 0.95 \\
$\lambda$ & 0.99 \\
Memory length & 30 steps \\
Learning time step & $0.05 \mathrm{~s}$ \\
\hline
\end{tabular}


required for the different disturbance cases in simulation to avoid damaging ABL-BI. Four different experimental cases are considered:

1. Walking experiment: no external disturbances are applied on the system. The robot walks on flat level ground to examine if balanced periodic walking is achieved.

2. Push disturbance: a push disturbance (pure force) is applied to the pelvis of the robot, while the robot is in single stance.

3. Step disturbance: a $1 \mathrm{~cm}$ step is placed under one foot of the robot.

4. Slope disturbance: the robot begins walking on flat level ground and transitions to walking on a $5^{\circ}$ slope.

These cases are presented in Sections 4.2.1 to 4.2 .4 respectively. Together, these different experimental cases can show if the BBLC is capable of generating new balancing strategies, using the behaviour set presented in Section 4.1. Moreover, additional testing is performed for the push disturbance case to examine how generalizable the learnt Q-table is to similar disturbances and to examine the behaviour of the controller when faced with transient disturbances.

\subsubsection{Walking}

The walking experiment was performed to examine if the BBLC could generated a stable - periodic walking pattern when no disturbances are present. Primarily this test examines if the unique three layer structure of the BBLC affects the controller's ability to generate a walking motion. No disturbances are applied to the robot during the disturbance and no Q-table learning was initially performed for this case. The default, ZMP-based walking control with an action vector of $\vec{A}=\left[\begin{array}{llllll}1 & 1 & 1 & 1 & 1 & 1\end{array}\right]^{T}$ was applied to the controller. The resulting successful walking motion is illustrated in Figure 4.3 , where a snapshot of each walking state is shown. The gait parameters are listed in Table 4.4 . 


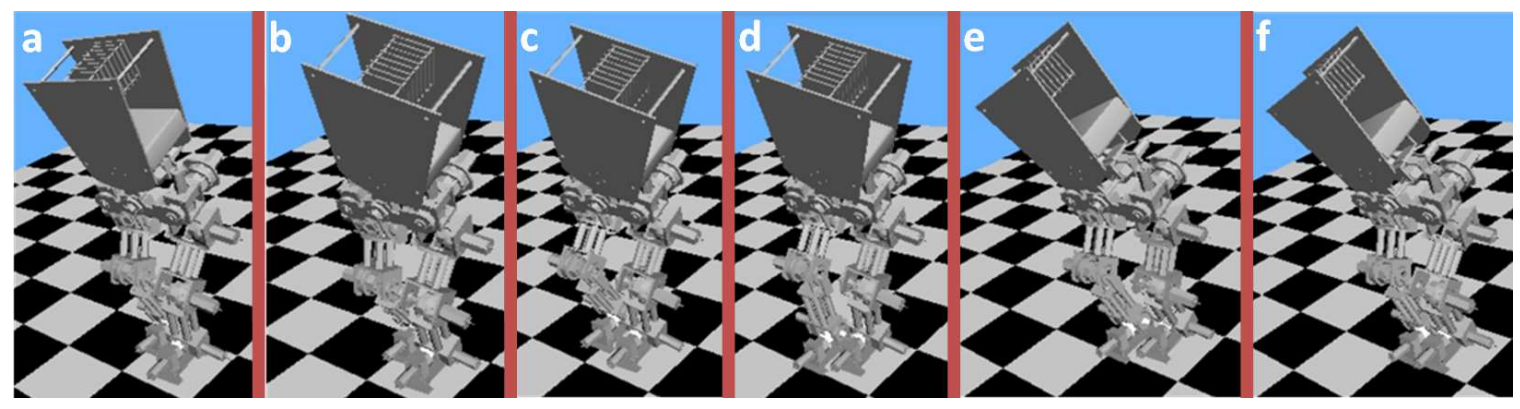

Figure 4.3: Freeze frame view of the biped during walking. (a) Double stance start; (b) weight shift to left foot; (c) right foot swing; (d) right foot down - double stance; (e) weight shift to right foot; (f) left foot swing.

The simulation was run for $100 \mathrm{~s}$ for a total of 17 steps. To examine if the motion is periodic, the phase plane plots and Poincaré map for the COM motion are shown in Figures 4.4 to 4.6 for the $\mathrm{X}, \mathrm{Y}$ and $\mathrm{Z}$ axis respectively. The Poincaré maps provide a more direct way of examining the periodicity of a phase plane trajectory. In the Poincaré map, rather than examining the entire trajectory of the phase plane motion, only one point in the trajectory is examined. In the bipedal balance application, the point that is examined coincides with a state change in the walking cycle, usually a transition from single to double stance. Qualitatively, if the The Poincaré points are clustered closely together, this indicates that the trajectory is periodic between cycles. Additionally, if a linear model of the system is available, the stability bound on the point cluster can be established. For the data presented, the Poincaré map for each cycle is taken at the left foot touch down (walking state 6 to 1). From these figures, we can see that after the first step, the phase variables for each axis settle and vary by less than $0.0001 \mathrm{~m}$ and $0.001 \mathrm{~m} / \mathrm{s}$ between cycles.

Table 4.4: Gait parameters used to plan walking motion of ABL-BI.

\begin{tabular}{|l|c|}
\hline Parameter & Value \\
\hline Stride length & $0.1 \mathrm{~m}$ \\
Stride height & $0.03 \mathrm{~m}$ \\
Swing time & $3 \mathrm{~s}$ \\
Double stance time & $3 \mathrm{~s}$ \\
\hline
\end{tabular}


(a)

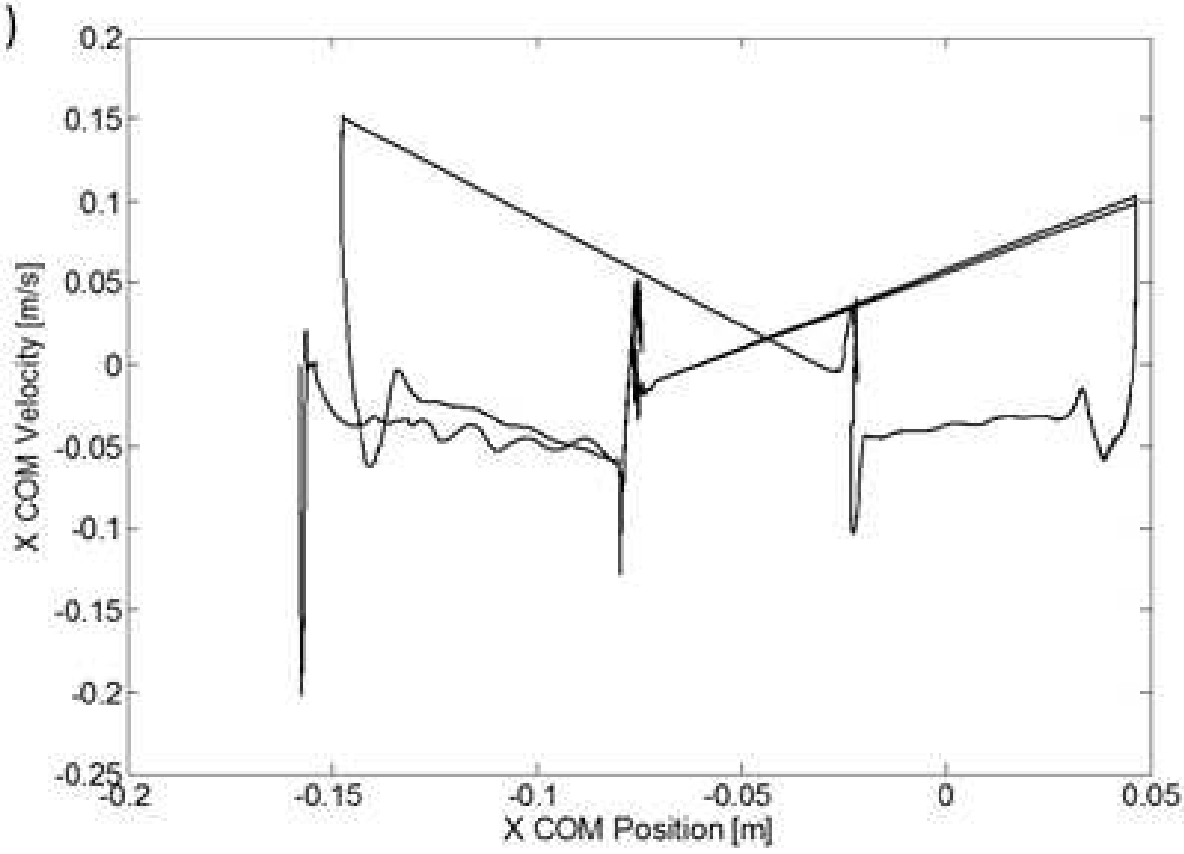

(b)

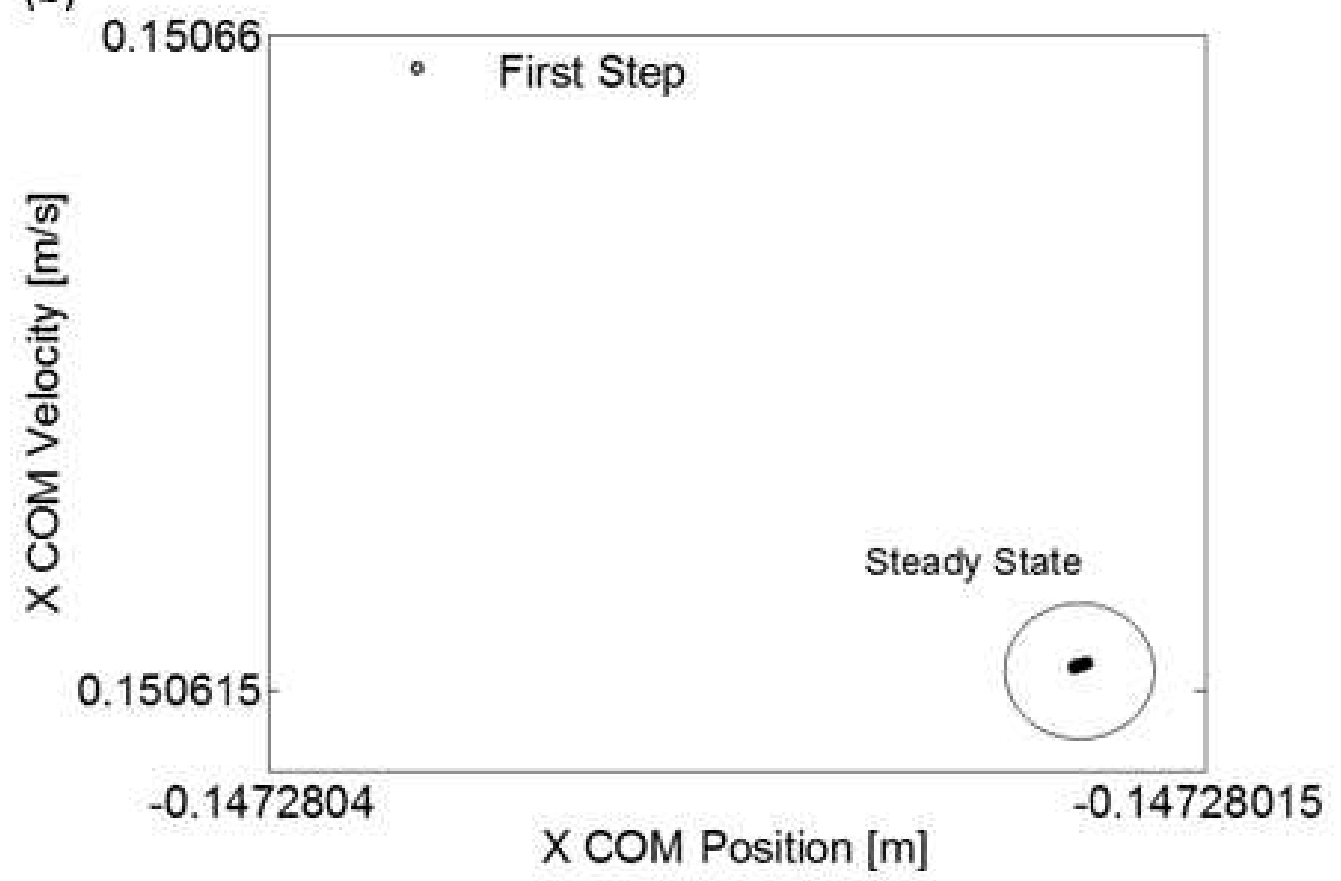

Figure 4.4: The phase plane of the $\mathrm{X}$ COM motion is shown in (a) and corresponding Poincaré map in (b) for a 17 step walking simulation. 
(a)
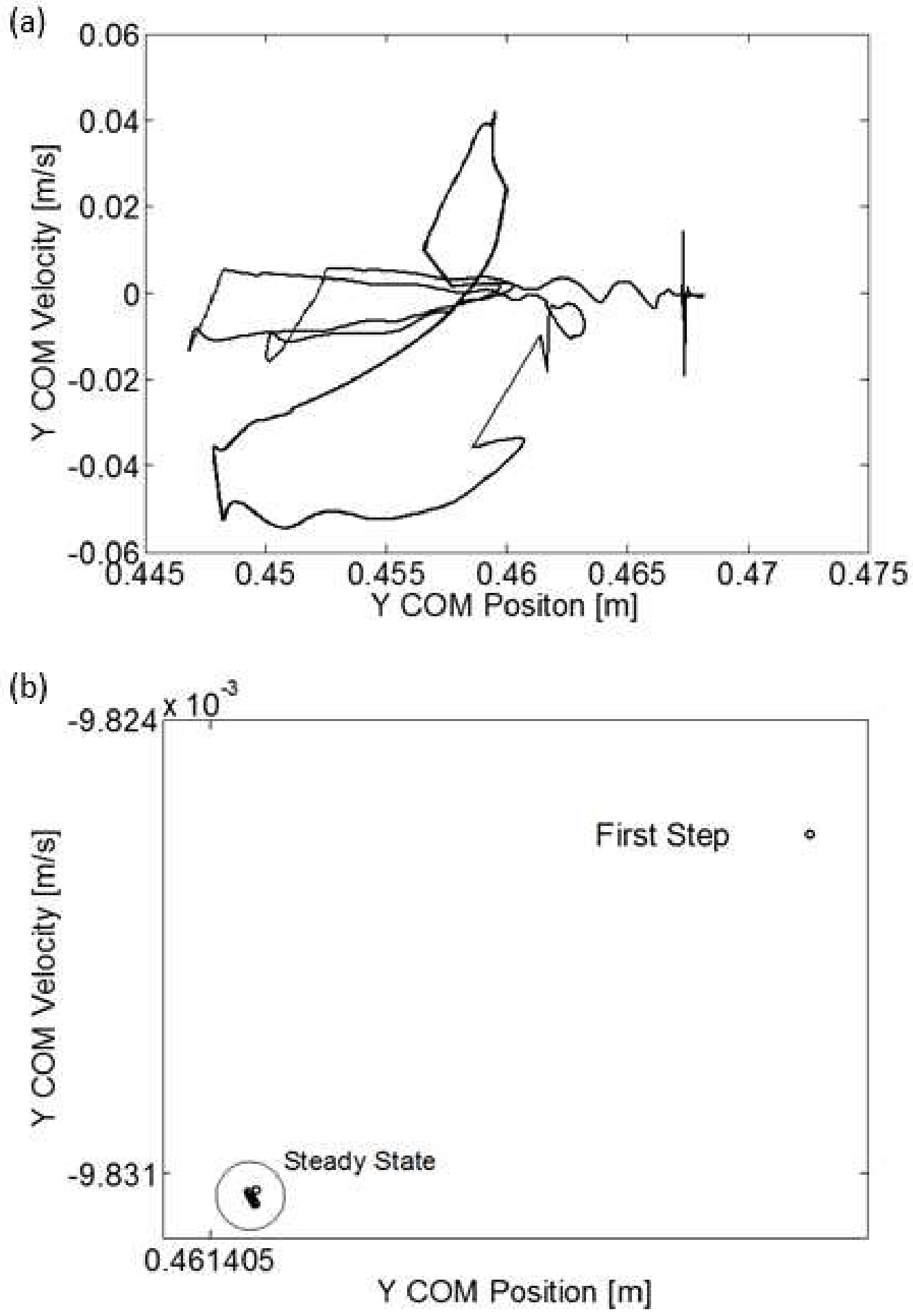

Figure 4.5: The phase plane of the Y COM motion is shown in (a) and corresponding Poincaré map in (b) for a 17 step walking simulation. 


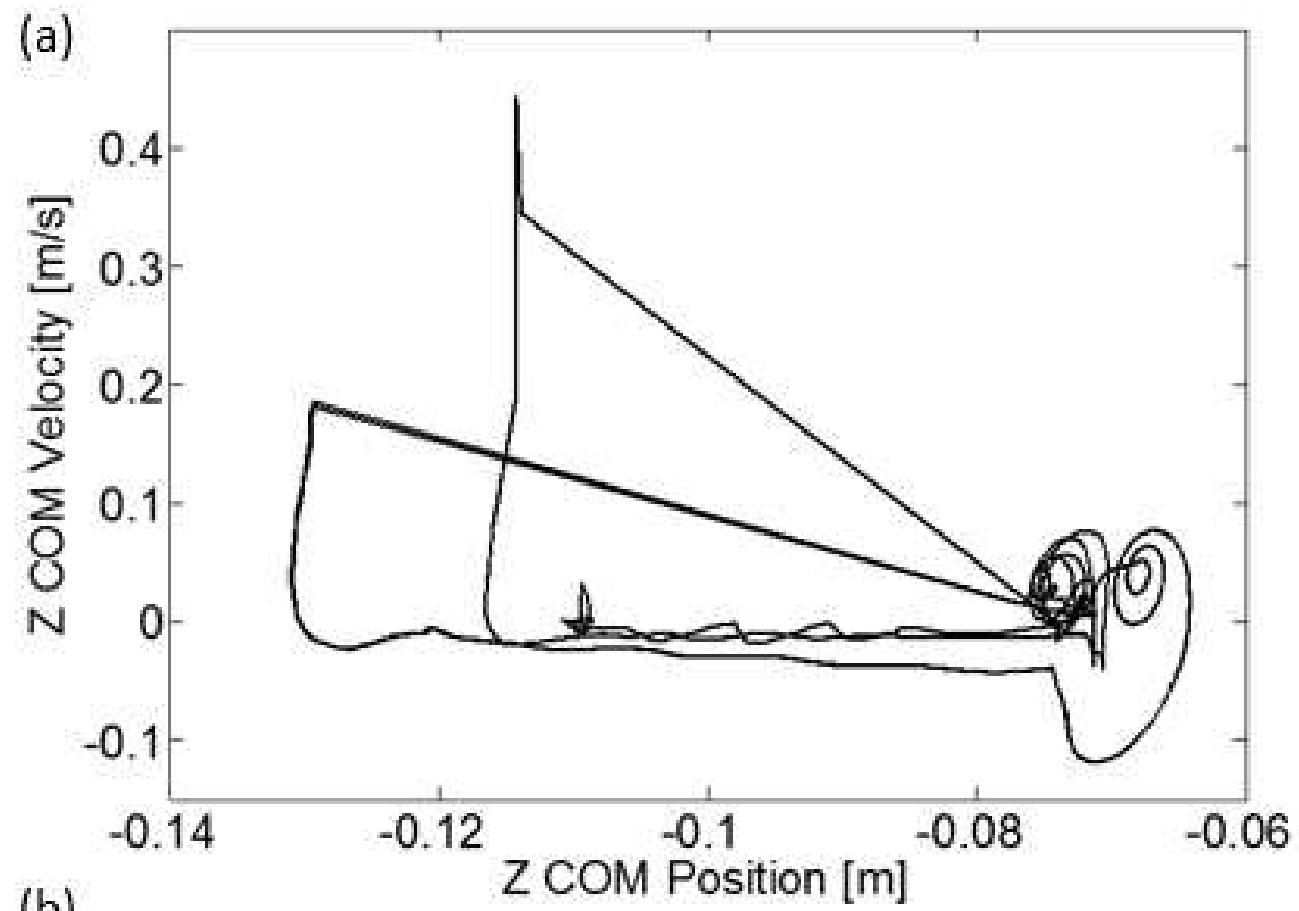

(b)

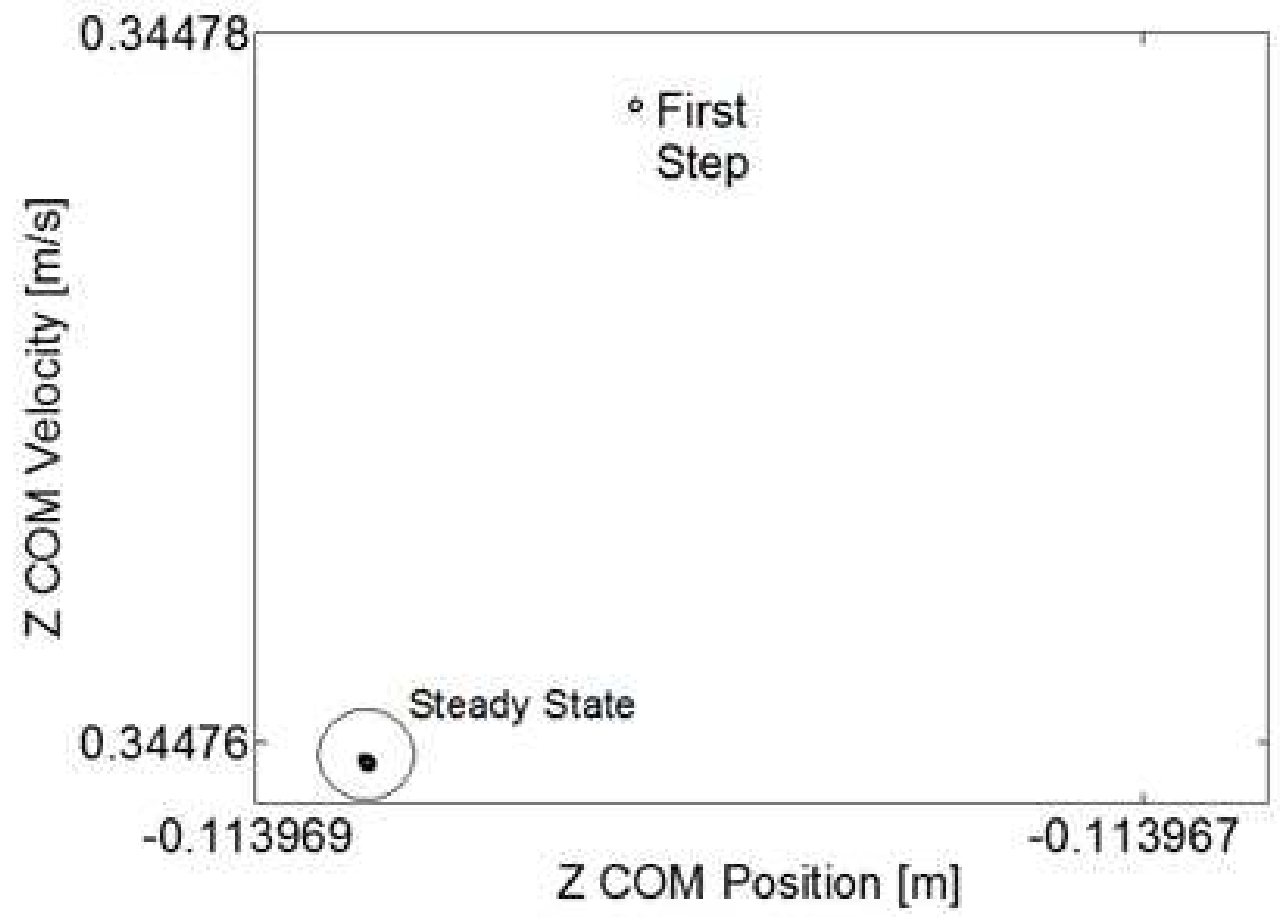

Figure 4.6: The phase plane of the $\mathrm{Z}$ COM motion is shown in (a) and corresponding Poincaré map in (b) for a 17 step walking simulation. 
This indicates the motion of the robot settles into a periodic motion which appears to be asymptotically stable and the walking motion would be continuously balanced given this repeated trajectory. One limitation of the resulting walking gait is slow walking speeds, requiring $12 \mathrm{~s}$ for a 2 step cycle. This is much slower than previous works and is primarily a result of mechanical design limitations. The pelvis width of ABL-BI is large compared to the body length, requiring large lateral weight shifts during double stance. Additionally, the mass distribution of ABL-BI is primarily concentrated in the legs (70\% of total mass, $30 \%$ in the torso). This mass distribution requires larger motions to perform lateral weight shifts, and also amplifies the dynamic effect of swinging a leg on the system. These effects combined together result in the primary limits on the walking speed of the system.

Overall, the key conclusions that we can draw from this first experiment is that the three layered structure of the BBLC can be used to generate a stable periodic walking motion that is similar to a standard controller structure, using a ZMP-based planning method.

\subsubsection{Push Disturbance}

The first disturbance that is examined is a push-type disturbance, where a disturbance force is applied to the pelvis of the robot during single stance. The objective of this experiment is to examine if the BBLC will find an emergent balancing strategy that will

compensate for this disturbance. Additionally, the generality of the learnt strategy is examined by varying the magnitude of the disturbance as compared with the disturbance applied in the initial learnt case. Finally, the behaviour of the BBLC is examined when the disturbance is transient, rather than continuous.

Initial Learning The first step is for the controller to learn a behaviour combination that will keep ABL-BI from falling. Initially a disturbance with a magnitude of $25 \mathrm{~N}$ along the $\mathrm{Z}$ axis and $15 \mathrm{~N}$ along the $\mathrm{X}$ axis is applied to the robot while the robot is in SSL. This causes 


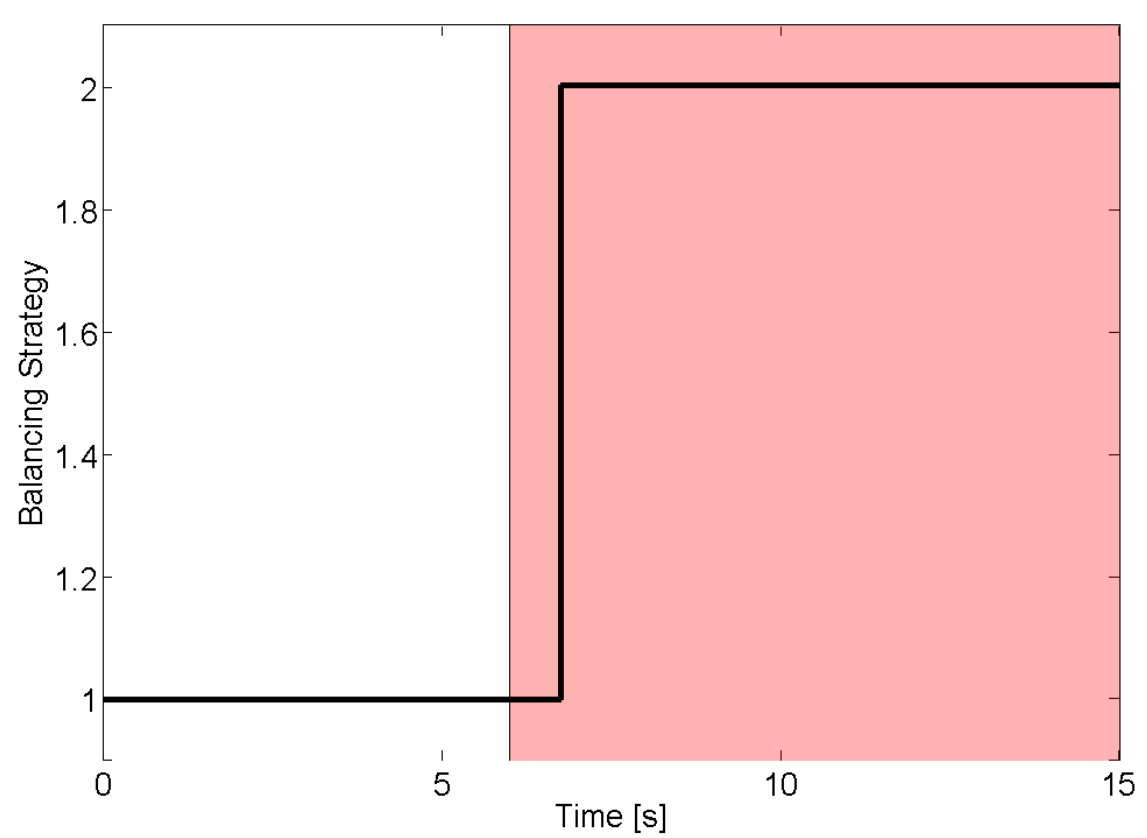

Figure 4.7: The balancing strategy being used in the BBLC controller where strategy 2 corresponds to an activation vector of $\vec{A}=\left[\begin{array}{lllll}1 & 3 & 2 & 3 & 2\end{array}\right]^{T}$ and strategy 1 to $\vec{A}=\left[\begin{array}{llllll}1 & 1 & 1 & 1 & 1 & 1\end{array}\right]^{T}$. The strategy is activated at $6.752 \mathrm{~s}$. The magnitude of the disturbance is $15 \mathrm{~N}$ in the $\mathrm{X}$ axis and $25 \mathrm{~N}$ in the $\mathrm{Z}$ axis (red area corresponds to the period when the disturbance is applied).

the robot to tip over if the default action vector is applied $\left(\vec{A}=[111111]^{T}\right)$. As a result, the Q-table is updated with a negative reward, associated with the state-space resulting from the disturbance being applied. When the same simulation is run again, the BBLC begins to search for a new behaviour combination that may balance the robot, under the same state conditions. This is illustrated in Figure 4.7, where the balancing strategy is applied at $6.752 \mathrm{~s}$, shortly after the disturbance is applied. The BBLC identifies that an unbalanced behaviour state is occurring and attempts a new behaviour combination (Note that for simplicity, the alternative balancing strategy will always have a value of 2 when plotted).

The learning algorithm iterates from $\vec{A}=\left[\begin{array}{llllll}1 & 1 & 1 & 1 & 1 & 1\end{array}\right]^{T}$ to $\vec{A}=\left[\begin{array}{llllll}3 & 3 & 4 & 3 & 5 & 5\end{array}\right]^{T}$ until a successful action vector is found. For this disturbance case, $\vec{A}=\left[\begin{array}{llllll}1 & 3 & 2 & 3 & 2 & 1\end{array}\right]^{T}$ was found to keep the robot balanced. When this action vector is activated by the BBLC, the swing leg motion is stopped, Y COM control is excluded and CMP control is activated for $\mathrm{X}$ 


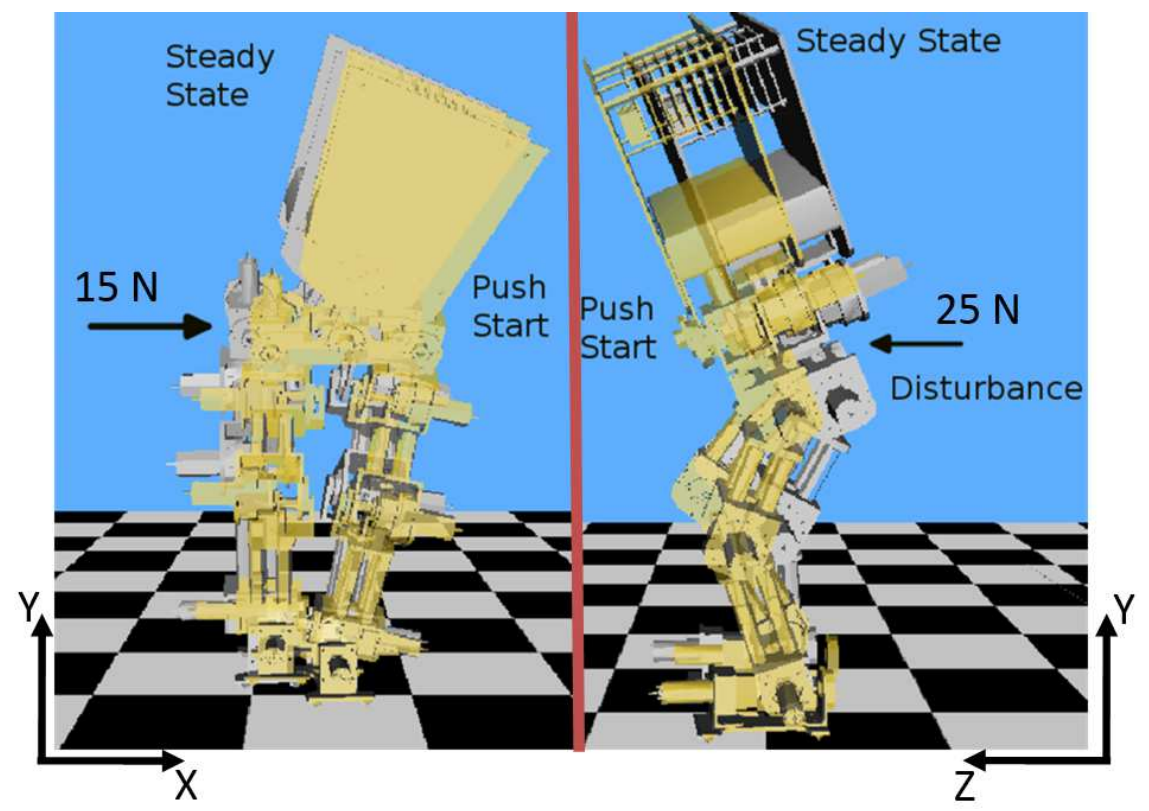

Figure 4.8: Motion of ABL-BI when a push is continually applied with a magnitude of 15 $\mathrm{N}$ in the $\mathrm{X}$ axis and $25 \mathrm{~N}$ in the $\mathrm{Z}$ axis. The initial position is shown in yellow, while the final steady state position is in grey. The motion of ABL-BI is against the direction of the disturbance.

(a)

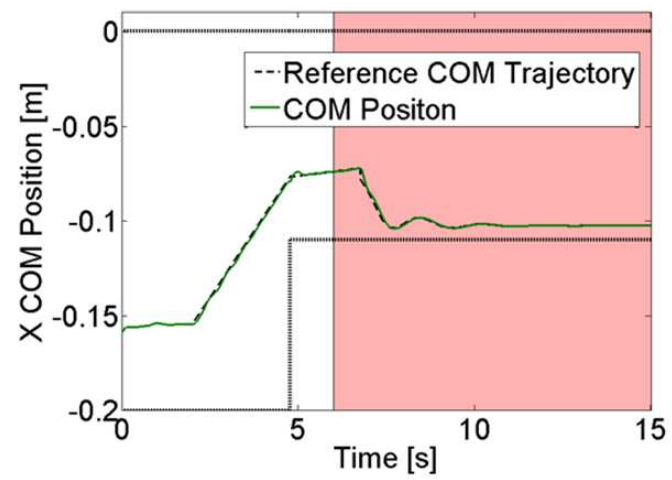

(b)

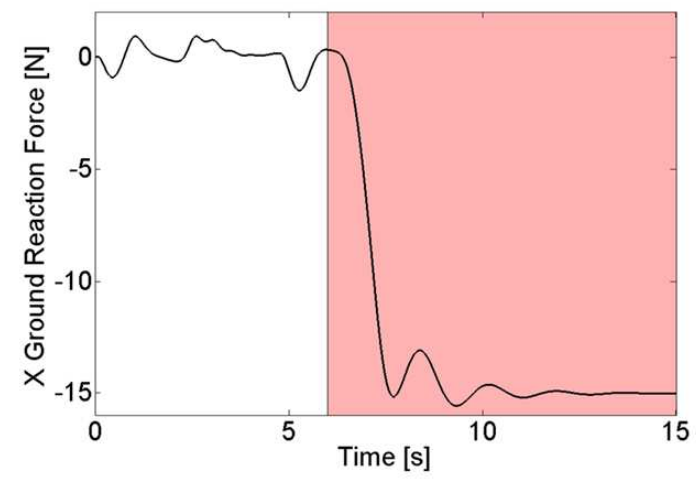

Figure 4.9: As a result of the push-type disturbance (red area corresponds to the period when the disturbance is applied) the CMP behaviour is activated for planning the COM motion. The resulting motion for $\mathrm{X} \mathrm{COM}$ is shown in (a) where the dotted boundary denotes the support polygon and the measured GRF in the X axis in (b). 
(a)

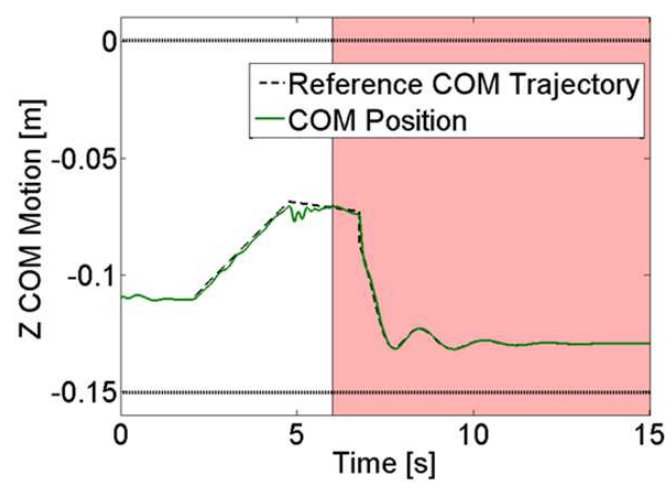

(b)

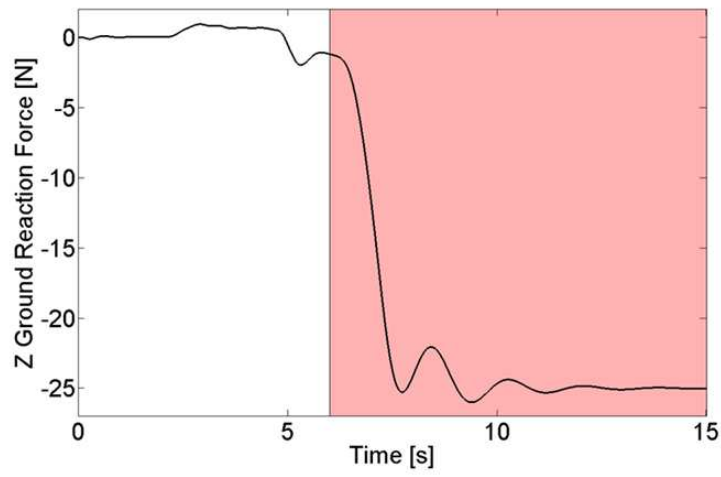

Figure 4.10: As a result of the push-type disturbance (red area corresponds to the period when the disturbance is applied) the CMP behaviour is activated for planning the COM motion. The resulting motion for Z COM is shown in (a) where the dotted boundary denotes the support polygon and the measured GRF in the $\mathrm{Z}$ axis in (b).

and Z COM motions. The resulting motion of the biped is shown in Figure 4.8. We can see that the overall motion of the biped is to move against the direction of the push force. This is reflected primarily in the X and Z COM motions, plotted in Figures 4.9 (a) and 4.10 (a). The reference COM motion diverges from the initial reference significantly after the disturbance is applied. This is the result of applying the CMP control law from Equations (4.4) and (4.5), where the reference COM motion is modified as a function of the lateral GRFs. These forces are shown in Figures 4.9 (b) and 4.10 (b) for the $\mathrm{X}$ and $\mathrm{Z}$ axes respectively. In steady-state, the measured GRFs settle to an equal but opposite magnitude of the disturbance force that is applied.

Extended Learning This initial learning phase demonstrated the basic functionality of the BBLC; an unknown disturbance was applied and the BBLC eventually learnt a new balancing strategy that was able to balance the robot under the same conditions. The next step is to examine how general the learning case is, i.e., will the newly found balancing strategy be activated under different conditions. A first test is to apply a similar push force, but with a different magnitude of $20 \mathrm{~N}$ in the $\mathrm{Z}$ axis and $10 \mathrm{~N}$ in the $\mathrm{X}$ axis. The resulting strategy activation is shown in Figure 4.11, where strategy 2 


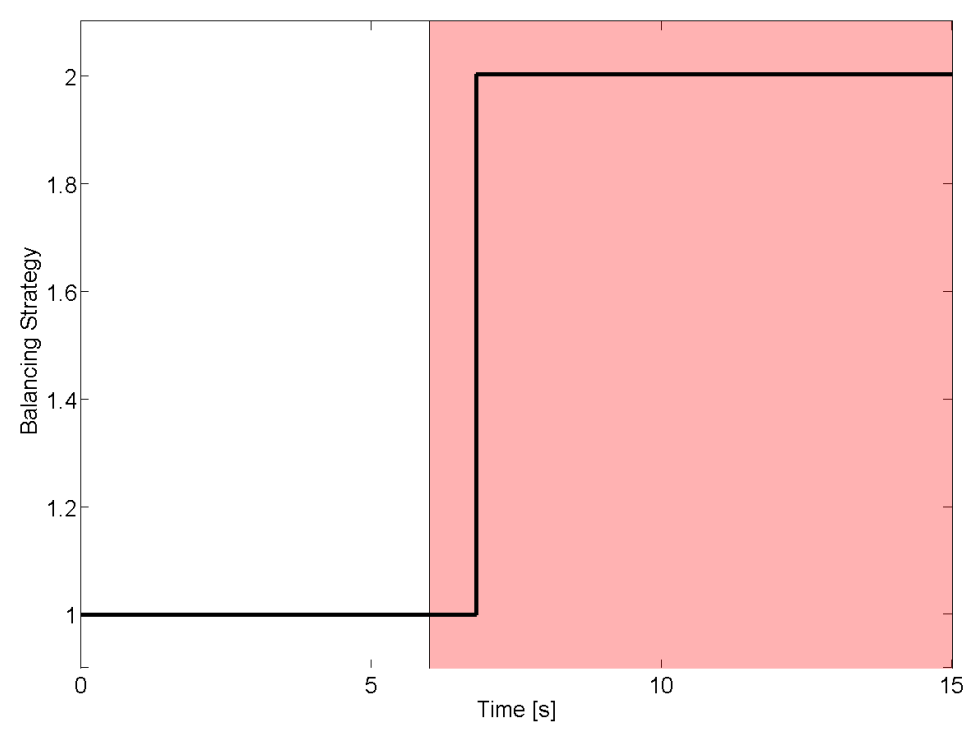

Figure 4.11: The balancing strategy being used in the BBLC controller where strategy 2 corresponds to an activation vector of $\vec{A}=[132321]^{T}$ and strategy 1 to $\vec{A}=[111111]^{T}$. The strategy is activated at $6.804 \mathrm{~s}$ and the magnitude of the disturbance (red area corresponds to the period when the disturbance is applied) is $10 \mathrm{~N}$ in the $\mathrm{X}$ axis and $20 \mathrm{~N}$ in the $\mathrm{Z}$ axis.

corresponds to $\vec{A}=\left[\begin{array}{lllll}1 & 3 & 2 & 3 & 2\end{array}\right]^{T}$. Compared to the behaviour activation in Figure 4.7 , the balancing strategy is activated $0.052 \mathrm{~s}$ later than for the originally-learnt case. However, it is still activated and keeps the robot balanced. This result shows that for different disturbance conditions, the initial learnt case still activated the balancing strategy. This experiment is repeated for a range of different $\mathrm{X}, \mathrm{Z}$ push magnitudes and the results are plotted in Figure 4.12. We can see that from one learnt case, the controller will activate the learnt balancing strategy to a larger set of disturbances with similar magnitudes. However, once the magnitude difference exceeds $10 \mathrm{~N}$, a new learning case will be required. Additionally there are many other variances which could be examined, including changing the disturbance position, varying the disturbance dynamically etc. To perform a truly exhaustive analysis of these possibilities may not practical. Rather, the BBLC is exposed to different disturbances types (step and slope) to establish if the algorithm can robustly compensate for other kinds of disturbances. 


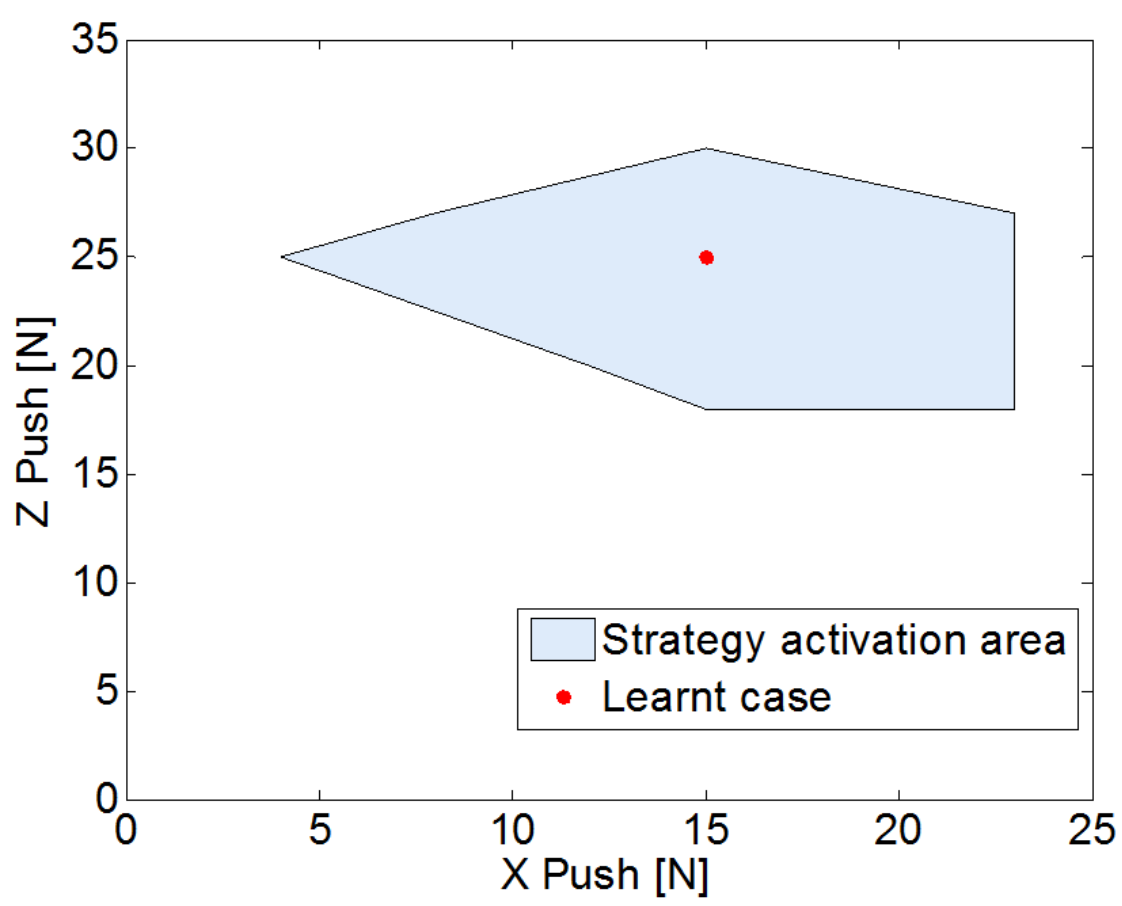

Figure 4.12: The area of push magnitudes under which a balancing strategy $(\vec{A}=$ $\left[\begin{array}{llllll}1 & 3 & 2 & 3 & 2 & 1\end{array}\right]^{T}$ ) is activated to maintain balance, given one learnt case.

If the disturbance force is applied during a different walking state such as in DBL, then the initially learnt case, learnt in SSL, is not sufficient to activate a proper balancing behaviour. What we can conclude from these results is that a specific learnt case provides some generality. If a disturbance of similar magnitude is applied during the same walking state, then one learning case is sufficient to activate the balancing strategy with $\vec{A}=$ $\left[\begin{array}{llllll}1 & 3 & 2 & 3 & 2 & 1\end{array}\right]^{T}$. However, for greater robustness to the disturbance, several more learning cases that cover a wider range of the state-space are needed. This is generally feasible in the simulation environment; however it may require proper planning from the designer and additional computational time. As an example, Figure 4.13 shows Q-values for $\vec{A}=$ $\left[\begin{array}{llllll}1 & 1 & 1 & 1 & 1 & 1\end{array}\right]^{T}$ of a learnt Q-table for varying magnitudes of push disturbances. Given that $Q \epsilon R^{5}$ the other variables are held constant for the surface plot shown $\left(0.01<S_{m}<0.01 \mathrm{~m}\right.$, $W S=3, \alpha_{e s t}=0^{\circ}$ ). This figure shows the learnt Q-values after exposing the learning algorithm to push-type disturbances ranging from -30 to $30 \mathrm{~N}$ for both the $\mathrm{X}$ and $\mathrm{Z}$ axis. A positive Q-value indicates that the BBLC has learnt for the state vector $\bar{s}$ corresponding 


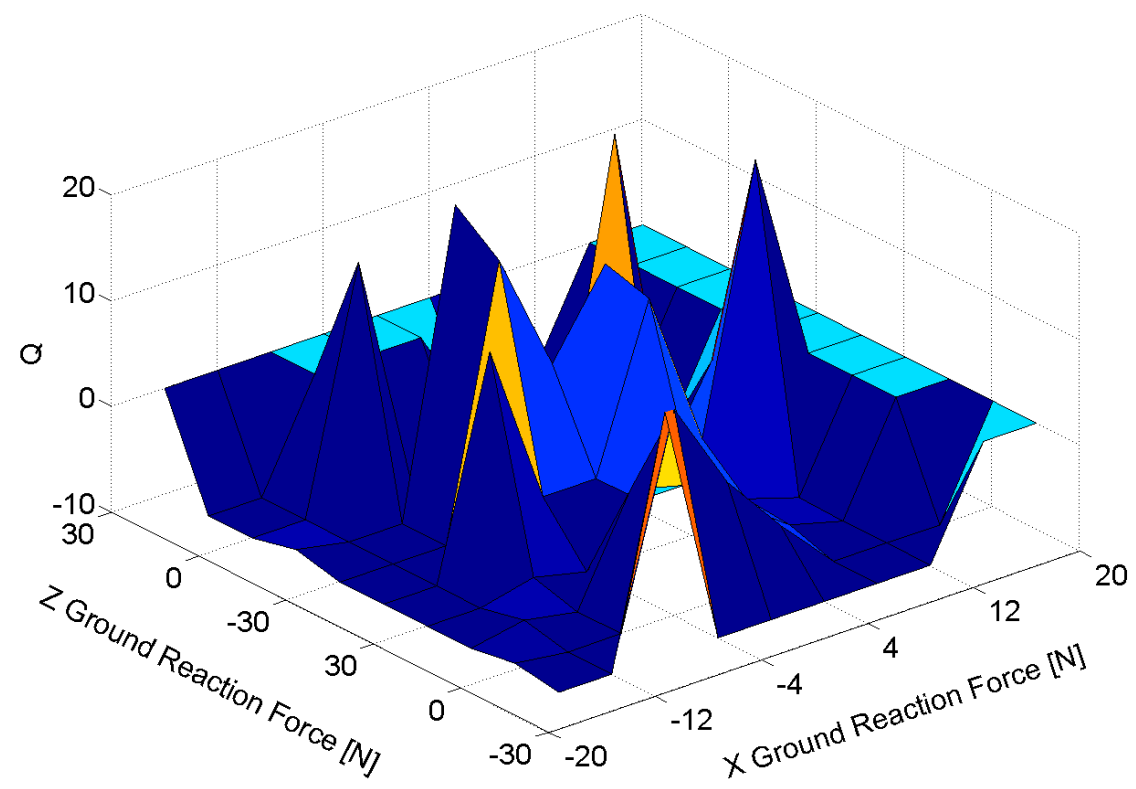

Figure 4.13: Surface plot showing Q-values as a function of the measured GRFs. All other states are held constant for this plot at values of $0^{\circ}$ for the slope estimate, Walking State $=3$, and stability margin $<0.01 \mathrm{~m}$.

to the Q-value, the robot is expected to remain balanced. Conversely, a negative Q-value indicates that a loss of balance is expected. For the area shown, the Q-values are mostly negative, indicating that the reinforcement learning algorithm has learnt for a wide range of push-type disturbances during single stance, with a stability margin less than $0.01 \mathrm{~m}$,

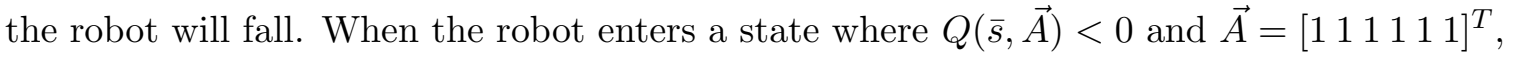
the algorithm will search for an alternate action vector $\vec{A}^{\prime}$ for which $Q\left(\vec{s}, \vec{A}^{\prime}\right)>0$. This will result in the activation of another balancing strategy which will have accumulated positive Q-values in the same region of the Q-table.

\section{Transient Disturbance}

A second extension that is examined for the push disturbance case is changing the nature of the push from a continuous to a transient push where the push is only applied for $5 \mathrm{~s}$. The resulting behaviour of the robot is shown in the strategy activation plot in Figure 4.14. For the period that the disturbance is applied, the balancing strategy is activated, similarly to the continuous case. Shortly after the disturbance is removed (0.752 s), the 


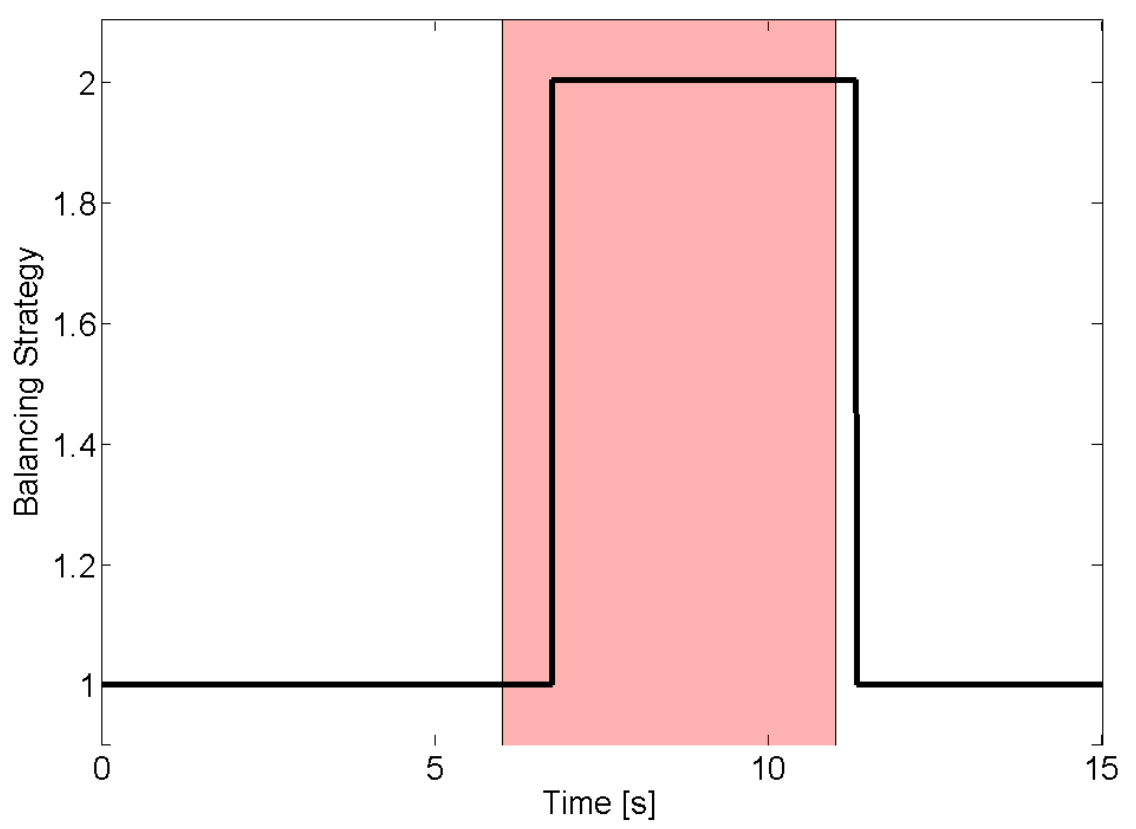

Figure 4.14: Balancing strategy activation for a transient disturbance (red area corresponds to the period when the disturbance is applied). The balancing strategy is activated $0.752 \mathrm{~s}$ after the disturbance is applied and deactivates $0.3050 \mathrm{~s}$ after the disturbance is removed.

BBLC recognizes a state change has occurred, the balancing strategy returns to the default walking action vector and normal walking resumes.

This result suggests that the state-space that was selected for the learning algorithm can effectively be used to identify when the push-type disturbance is being applied. Also, the controller structure allows the robot to return to normal walking state after the disturbance is removed.

\subsubsection{Step Disturbance}

The second disturbance case that is examined is placing a small step under one of the feet. Similarly to the push disturbance, the robot starts with no knowledge of the disturbance. When the default action vector is used, a fall occurs and negative reward is accumulated in the Q-table. The learning algorithm begins to search for new behaviour combinations 


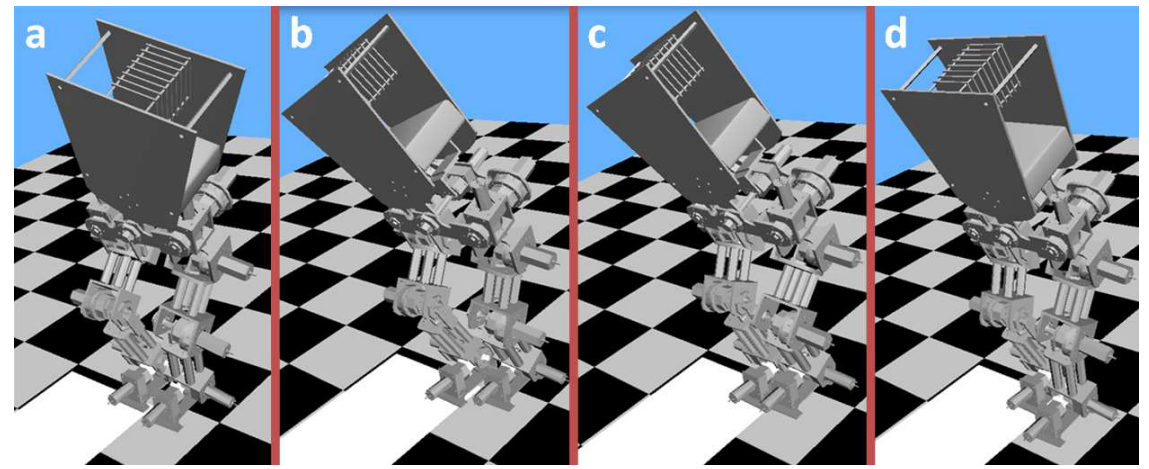

Figure 4.15: Walking motion of ABL-BI when a $1 \mathrm{~cm}$ step is placed under the right foot: (a) DBL; (b) DBLS; (c) SSL; (d) DBR.

(a)

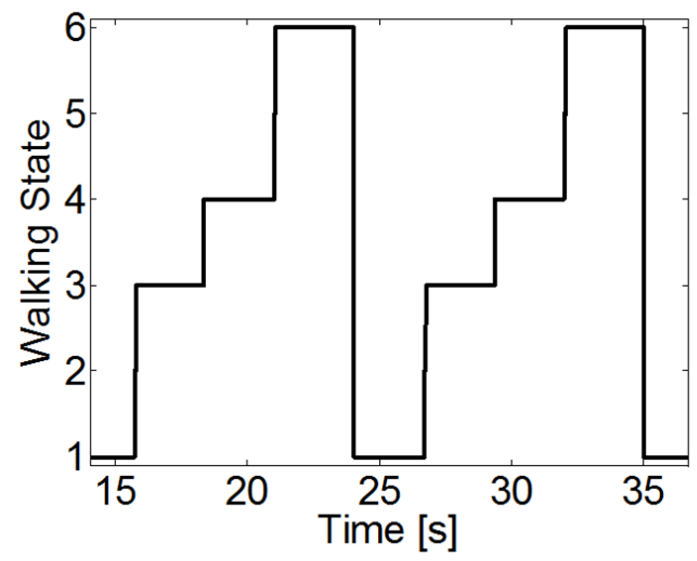

(b)

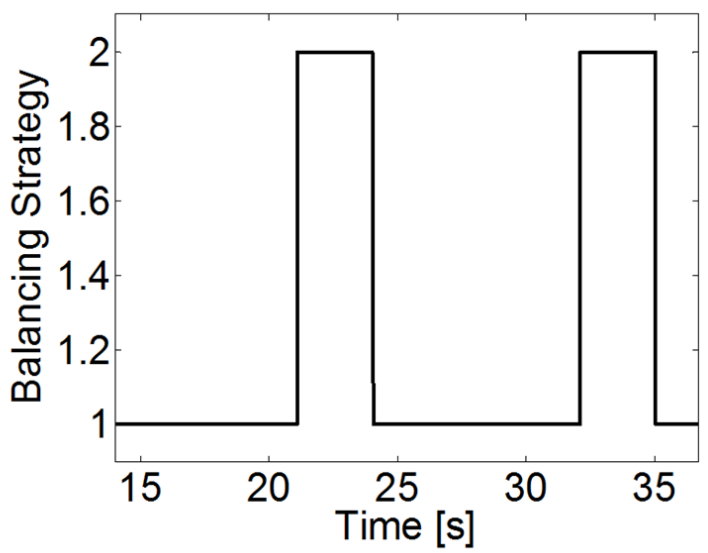

Figure 4.16: Activation of the balancing strategy when walking with a $1 \mathrm{~cm}$ step under the right foot in (b). The balancing strategy activation coincides with the robot being in single stance on the right foot where the walking state is shown in (a). 
until a successful balancing strategy is found. For the step disturbance, an action vector of $\vec{A}=\left[\begin{array}{lllll}1 & 1 & 2 & 3 & 2\end{array}\right]^{T}$ is found to maintain balance on steps of up to $1 \mathrm{~cm}$ in height. In this strategy, Y COM control is excluded and $\mathrm{X}$ and $\mathrm{Z}$ COM motions change to using CMP based planning. The resulting motion is illustrated in Figure 4.15. The balancing strategy activation is shown in Figure 4.16 (b).

There are two notable features with the results shown in this case. The first is that, unlike for the push-type disturbance, the new balancing strategy is not continuously applied. In Figure 4.16 (b), the balancing strategy is only activated periodically, and then returns to the default walking action vector. Comparing to the walking state plot in Figure 4.16 (a), the strategy activation coincides with the robot being in Walking State $=6$, which corresponds to single stance, where the left foot is swinging. Therefore, the BBLC has learnt that during this period in the walk, given the state-space that occurs when a step is present, the balancing strategy must be applied, otherwise normal walking is required. If the newly applied balancing strategy $\left(\vec{A}=\left[\begin{array}{llllll}1 & 1 & 2 & 3 & 2 & 1\end{array}\right]^{T}\right)$ is applied continuously for all walking states, a fall will occur. This is primarily a result of excluding the Y COM control from the motion control. As a result, the COM height continues to drop until a fall occurs as shown in Figure 4.17. This is a significant result, given that the BBLC learning algorithm not only learnt that a balancing strategy needed to be activated, but also deactivated for walking states other than SSR. The second key feature of this result is that the step is not reflected in any of the states directly; i.e., none of the state variables are directly related to the step height of the foot. The controller simply infers that an unstable situation is occurring from the measured reduced state-space.

Similarly to the push-type disturbance, the generality of the learnt example is dependent on the state-space definition. It was found that the behaviour would be activated for steps greater than $0.4 \mathrm{~cm}$. However, if the step is placed under the left foot, rather than the right, a new learning case is needed. 
(a)

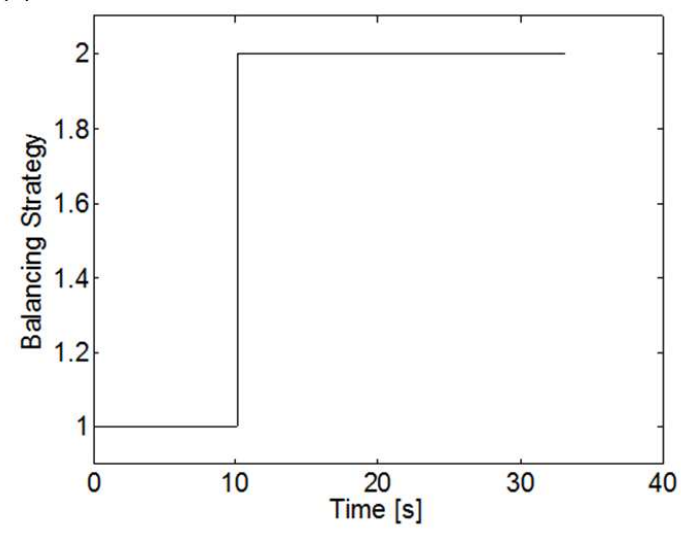

(b)

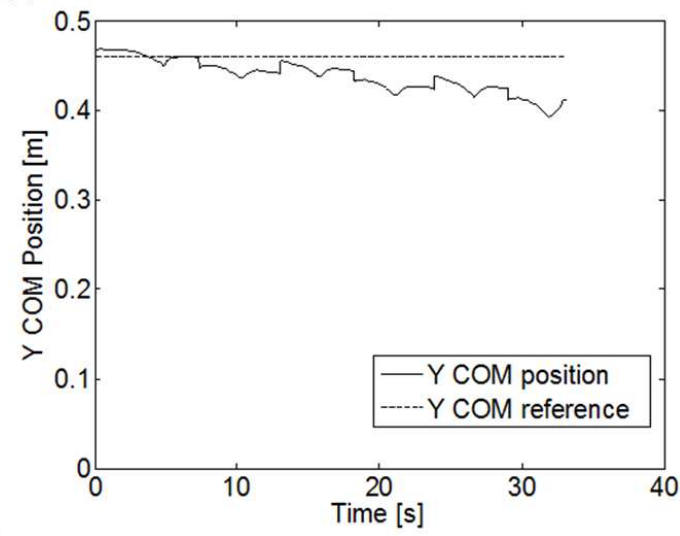

Figure 4.17: Balancing strategy is artificially kept activated in (a) causing a continual drop in the Y COM position in (b), eventually resulting in a fall.

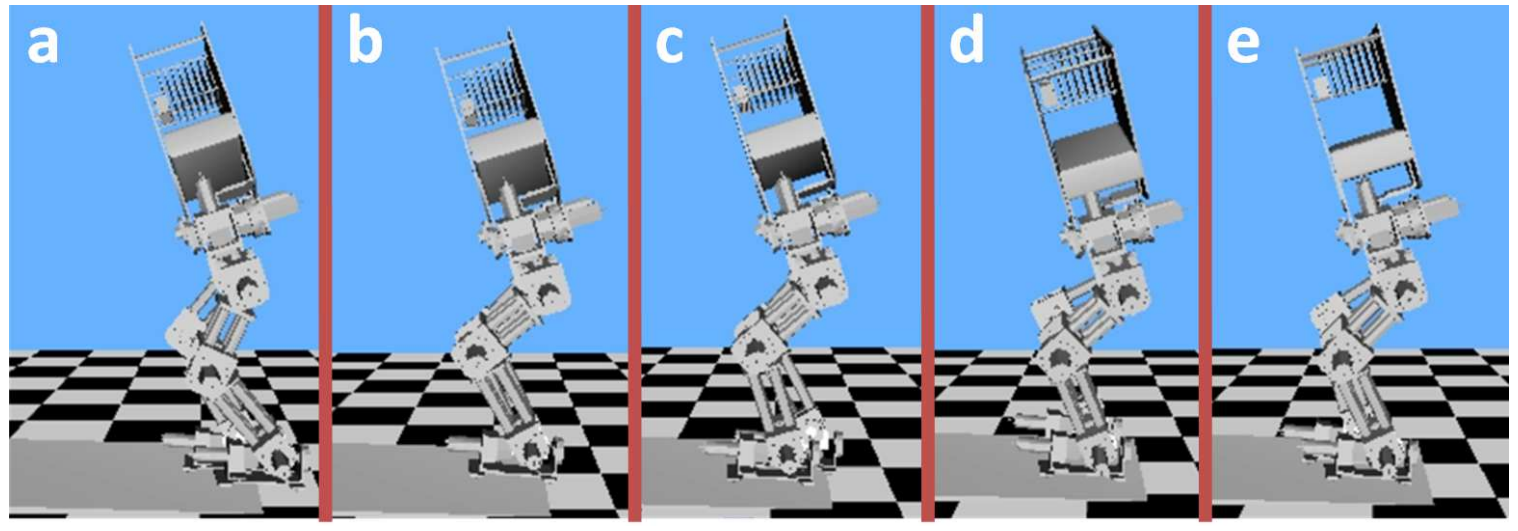

Figure 4.18: Motion of ABL-BI when walking up a $5^{\circ}$ slope: (a) double stance; (b) left foot swing; (c) double stance; (d) right foot swing; (e) double stance. 


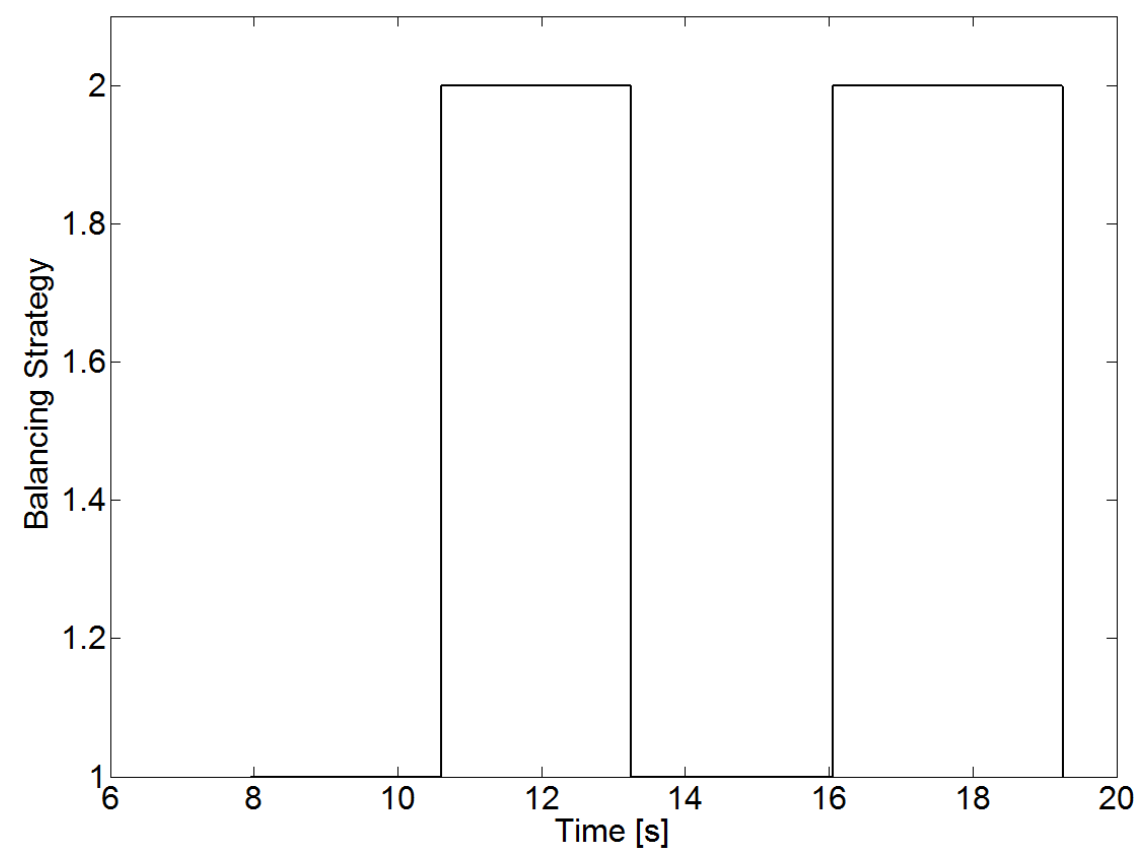

Figure 4.19: Balancing strategy activation when walking on a $5^{\circ}$ slope. The balancing strategy activation coincides with single stance for both the right and left foot.

\subsubsection{Slope Disturbance}

The last test case that is examined is walking on a slope. ABL-BI begins on flat level ground and beings stepping on a $5^{\circ}$ slope after the first step. Again the controller begins with no a priori knowledge of the disturbance, and must learn a new balancing strategy to

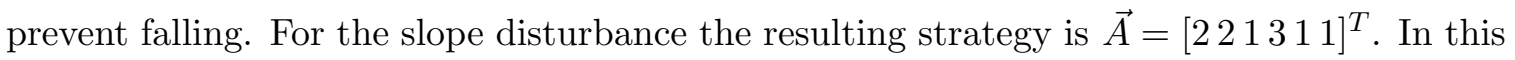
strategy the controller uses the slope estimating behaviour for the foot placement, a longer stride length for the gait parameter, and excludes the Y COM control. The resulting motion of the biped walking up a $5^{\circ}$ slope is shown in Figure 4.18, with the behaviour activation shown in Figure 4.19. For the slope disturbance, the balancing strategy is activated when the biped is in single stance, when either foot is swinging. Hence, during single stance, the Y COM motion is not controlled, the foot stride is extended and the landing angle of the foot is set to the slope estimate, shown in Figure 4.20. After the first step onto the slope at $7.51 \mathrm{~s}$, the slope estimate jumps from $0^{\circ}$ to above $5^{\circ}$ and then settles to $4.9^{\circ}$ afterwards. 


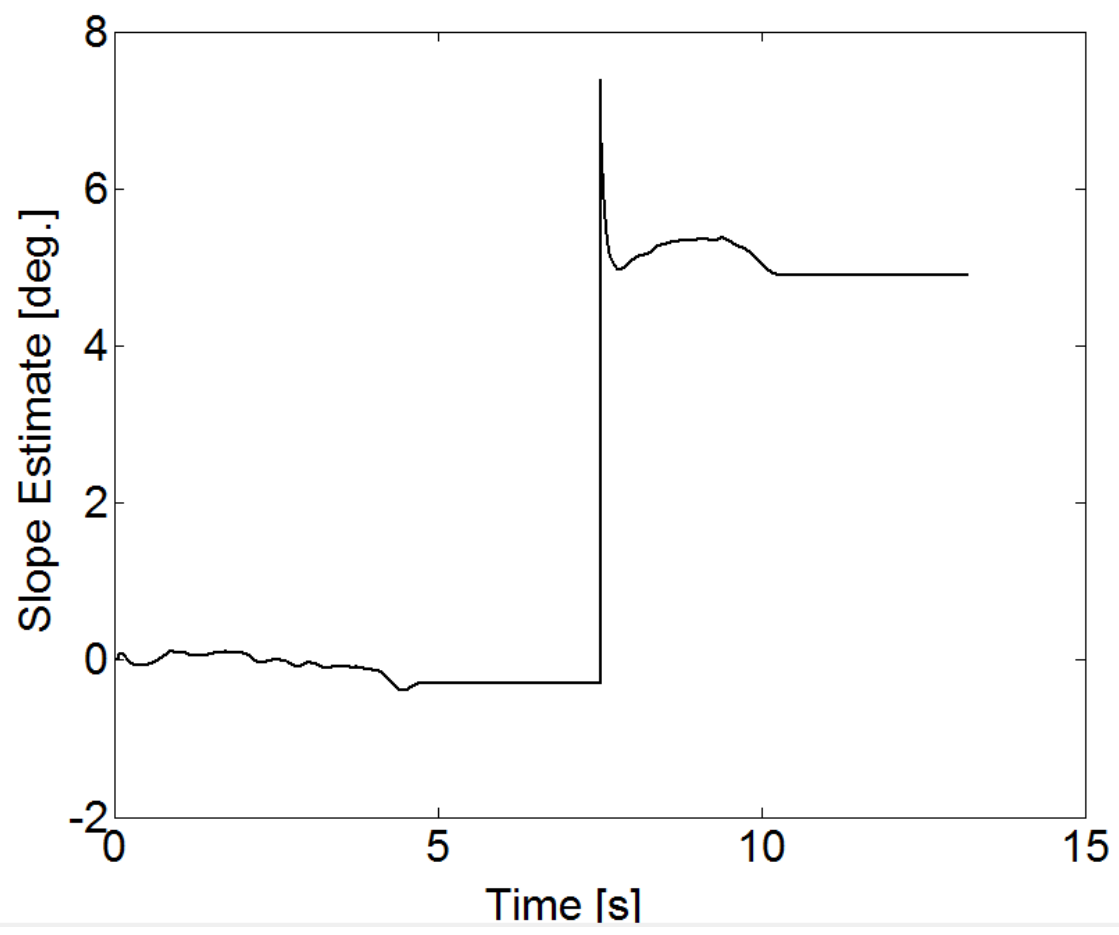

Figure 4.20: The slope estimate for a complete walking cycle where the robot transitions from a flat to sloped terrain.

Overall this result is similar to the step-type disturbance. The robot walks on modified terrain and a balancing strategy is periodically applied to maintain balance. However, for this disturbance there is some a priori planning in the controller given that a slope estimating behaviour is included for the foot angle task-space motion. However, this

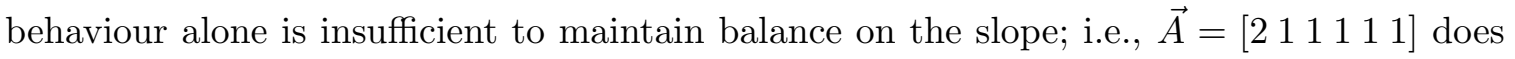
not result in balanced walking. Hence, the additional behaviours and the overall structure of the BBLC is required to find a new balancing strategy.

\subsection{Chapter Summary}

In this chapter, the implementation of the BBLC to control the ABL-BI robot was presented along with simulation results, summarized in Table 4.5, of four different cases: (1) walking 
without disturbance, (2) a push-type disturbance, (3) walking with a step under one foot and (4) walking up a slope. The result for the walking case showed that the three layered structure of the BBLC did not affect the controller's ability to generate a stable walking gait, when using a common ZMP walking planner. For each of the three disturbance cases, a new balancing strategy capable of maintaining the robot's balance emerged. As with the push disturbance case, the emergent strategy can sacrifice other control objectives, such as walking speed or energy efficiency to favour balance. Collectively, the results to the three disturbance cases support the core hypothesis of the BBLC - combining multiple balancing behaviours can result in emergent balancing strategies to unknown disturbances. The results also show that the reinforcement learning layer, with the pre-defined reduced state-space, was able to classify the unknown disturbances and find a balancing strategy to compensate for it.

Additionally, for the push disturbance case, the results showed the generalizability of a single learning case. It showed that for a disturbance within $\pm 10 \mathrm{~N}$ of the originally learnt case, the learnt behaviour strategy would be activated. However, for a disturbance with magnitudes diverging by more than $10 \mathrm{~N}$ from the learnt case, or occurring during a different walking state, a new learning case would be required. Finally the slope case showed that although the controller effectively generates new balancing strategies, it is

Table 4.5: Summary of simulation results for 4 different cases that were examined.

\begin{tabular}{|c|c|c|c|}
\hline Case & $\begin{array}{r}\text { Learnt balancing } \\
\text { strategy }\end{array}$ & $\begin{array}{r}\text { Maximum disturbance } \\
\text { before learning }\end{array}$ & $\begin{array}{r}\text { Maximum disturbance } \\
\text { after learning }\end{array}$ \\
\hline $\begin{array}{l}\text { Periodic } \\
\text { walk }\end{array}$ & $\vec{A}=\left[\begin{array}{llllll}1 & 1 & 1 & 1 & 1 & 1\end{array}\right]^{T}$ & $\mathrm{~N} / \mathrm{A}$ & $\mathrm{N} / \mathrm{A}$ \\
\hline $\begin{array}{l}\text { Push } \\
\text { disturbance }\end{array}$ & $\vec{A}=\left[\begin{array}{llllll}1 & 3 & 2 & 3 & 2 & 1\end{array}\right]^{T}$ & $F_{x}= \pm 10 \mathrm{~N}, F_{z}= \pm 20 \mathrm{~N}$ & $F_{x}= \pm 15 \mathrm{~N} F_{z}= \pm 30 \mathrm{~N}$ \\
\hline $\begin{array}{l}\text { Step } \\
\text { disturbance }\end{array}$ & $\vec{A}=\left[\begin{array}{llllll}1 & 1 & 2 & 3 & 2 & 1\end{array}\right]^{T}$ & $0.002 \mathrm{~m}$ & $0.01 \mathrm{~m}$ \\
\hline $\begin{array}{l}\text { Slope } \\
\text { disturbance }\end{array}$ & $\vec{A}=\left[\begin{array}{llllll}2 & 1 & 1 & 1 & 1 & 1\end{array}\right]^{T}$ & $0^{\circ}$ & $5^{\circ}$ \\
\hline
\end{tabular}


ultimately still limited by the pre-planned behaviours and definition of the state-space. Therefore, some a priori planning is still required. However, given the controller structure, it can be easily scaled to include new behaviours, of any complexity, to augment the balance control of the robot. 


\section{Chapter 5}

\section{Experiments with ABL-BI}

In Chapter 4, the BBLC was implemented on a 3D dynamic simulation model of ABL-BI. The results from Chapter 4 indicated that the BBLC is capable of generating emergent balancing strategies to unknown disturbances. In this chapter, the results of implementing the BBLC on the ABL-BI platform are presented. The primary objective of these results is to validate experimentally the results found in Chapter 4. More precisely, the following research questions are examined:

1. Can the Q-table learnt in simulation be transferred to the experimental platform with equivalent results?

2. Are the same balancing strategies activated on ABL-BI as in simulation?

3. Do the emergent balancing strategies compensate for the applied disturbances as in the simulation?

To answer these research questions, the experiments reproduced the same four cases that were examined in simulation as listed below:

1. Periodic walking experiment to examine if a balanced cyclic walking gait can be generated.

2. Push disturbance experiment where ABL-BI is exposed to a push disturbance applied at the pelvis of the robot. 
3. Step experiment where a $1 \mathrm{~cm}$ step is placed under one of the feet of ABL-BI while it is walking.

4. Slope experiment where a $5^{\circ}$ slope is placed in ABL-BI's path.

For these experiments, the implementation of the BBLC is identical to the implementation in simulation described in Section 4.1. In other words, the controller is composed of the same task-space motions and balancing behaviours. In the proposed experiments, no learning occurs as the Q-table which was learnt via simulation is transferred to ABL-BI. The primary difference with moving from the simulated to experimental ABL-BI is the variation between the two. In the simulation, dynamic and kinematic parameters were known exactly and sensors gave perfect information. Additionally, there is no direct measurement of the lateral GRFs. As a result, an estimator is designed to estimate these forces and is described in Section 5.1. The results for the four experiment cases listed above follow in Section 5.2. Finally, a chapter summary is presented in Section 5.3 .

\subsection{Estimation of Ground Reaction Forces}

In simulation, the GRFs are taken directly from the simulation model. Therefore, the controller has perfect knowledge of the normal ( $\mathrm{Y}$ axis) GRF and lateral GRFs (X and $\mathrm{Z}$ axes). ABL-BI has four force sensors on each foot, each measuring the normal force component of the GRF only, as described in Chapter 2. This allows for a measurement of the normal force Y GRF and COP, but the $\mathrm{X}$ and $\mathrm{Z}$ components of the GRFs (shear forces) are not measured directly (note that in other bipedal robots, a measurement of the GRFs and COP is achieved by placing a 6-axis force torque sensor in the ankle). By using the COP measurement in combination with the LIPM, an estimator can be designed to estimate these lateral GRFs. From (1.1) and (1.2). If no external disturbances are acting 
on the system, the ZMP of the biped is defined as

$$
\begin{aligned}
& x_{Z M P}=x_{C O M}-\frac{\ddot{x}_{C O M} y_{C O M}}{g}, \\
& z_{Z M P}=z_{C O M}-\frac{\ddot{z}_{C O M} y_{C O M}}{g},
\end{aligned}
$$

and the ZMP should be equivalent to the COP position. The COM position, $x_{C O M}, y_{C O M}, z_{C O M}$ are known from the forward kinematics. Similarly the COM accelerations are estimated from the forward kinematics as defined in (2.2). Hence, if the ZMP and COP are not coincident, there are three possibilities: (1) there is a measurement error in the COP, (2) there is a modelling error of the COM position, or (3) a disturbance is acting on the system. An estimator of the GRFs can be defined as

$$
\begin{aligned}
& F_{x}=K_{x}\left(x_{Z M P}-x_{C O P}\right)+C_{x}, \\
& F_{z}=K_{z}\left(z_{Z M P}-z_{C O P}\right)+C_{z},
\end{aligned}
$$

where $K_{x}, K_{z}, C_{x}, C_{z}$ are constants that are determined through calibration. The constant error between the model and measured ZMP will be accounted for by $C_{x}$ and $C_{z}$. These constants will also account for errors occurring due to possibilities (1) and (2) listed above. Therefore, any remaining divergence between the ZMP and COP will be the result of an external force acting on the system. A similar approach was used by Sugihara 137 where he also used the error between the measured COP and estimated ZMP. However, in this implementation, only modelling errors were being estimated to generate a more accurate estimate of the ZMP. The estimator defined by (5.3) and (5.4) was initially examined in simulation where the exact disturbance, and GRFs are known. Figure 5.1 shows a comparison of an estimated GRF and directly measured GRF from the model. 
(a)

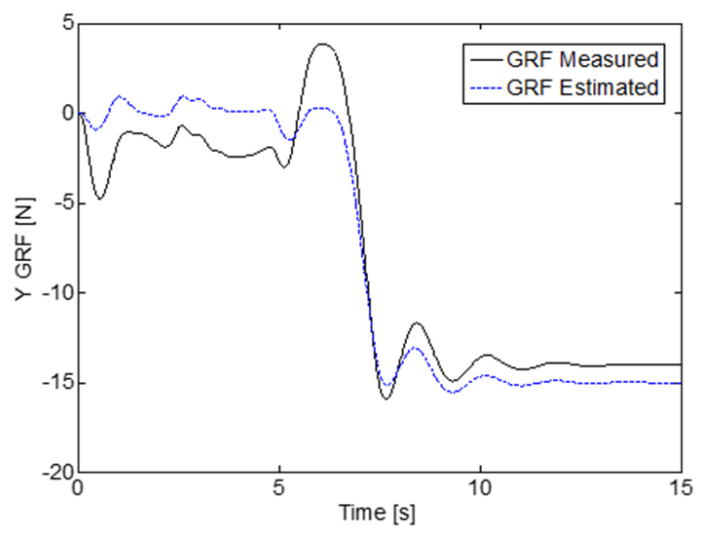

(b)

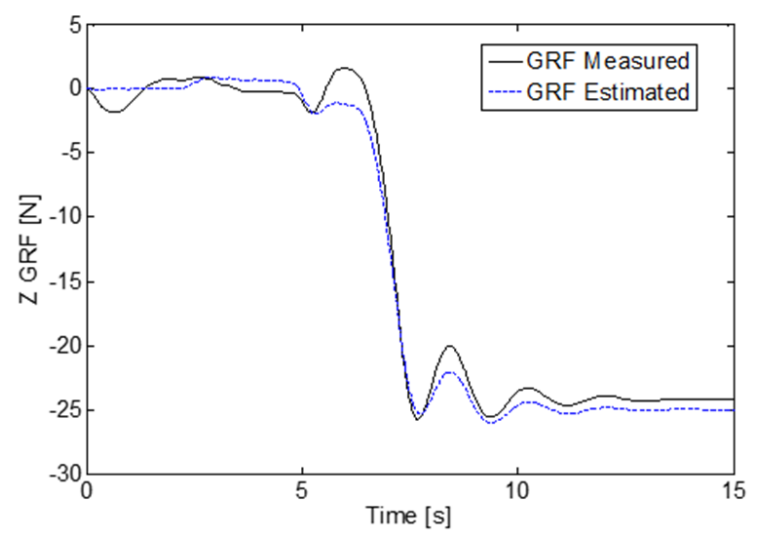

Figure 5.1: Comparison of the measured and estimated GRFs from simulation for both the $\mathrm{X}$ axis in (a) and $\mathrm{Z}$ axis in (b). The applied disturbance is $15 \mathrm{~N}$ in the $\mathrm{X}$ axis and $25 \mathrm{~N}$ in the $\mathrm{Z}$ axis.

On average, the error between both the actual and estimated GRF is found to be $6.9 \%$ after calibration of the estimator. However, as seen in Figure 5.1 there is a constant offset between the measured and estimated shear forces. This is primarily a result of keeping $C_{x}$ and $C_{z}$ constant. The constant offset between the estimated ZMP and measured COP is a function of the joint angles $\theta$. Therefore this offset will vary in different poses of the robot. However, in the case shown in Figure 5.1, this error does not result in a significant error in the estimated GRF.

To calibrate the GRF estimator, a known force is applied to the pelvis of the robot as shown in Figure 5.2, where the disturbance is applied via a set of weights of known mass.

Table 5.1: Calibrated GRF estimator parameters.

\begin{tabular}{|lr|}
\hline Parameter & Value \\
\hline$K_{x}$ & -404 \\
$C_{x}$ & 9.0 \\
$K_{z}$ & -333 \\
$C_{z}$ & -4.2 \\
\hline
\end{tabular}




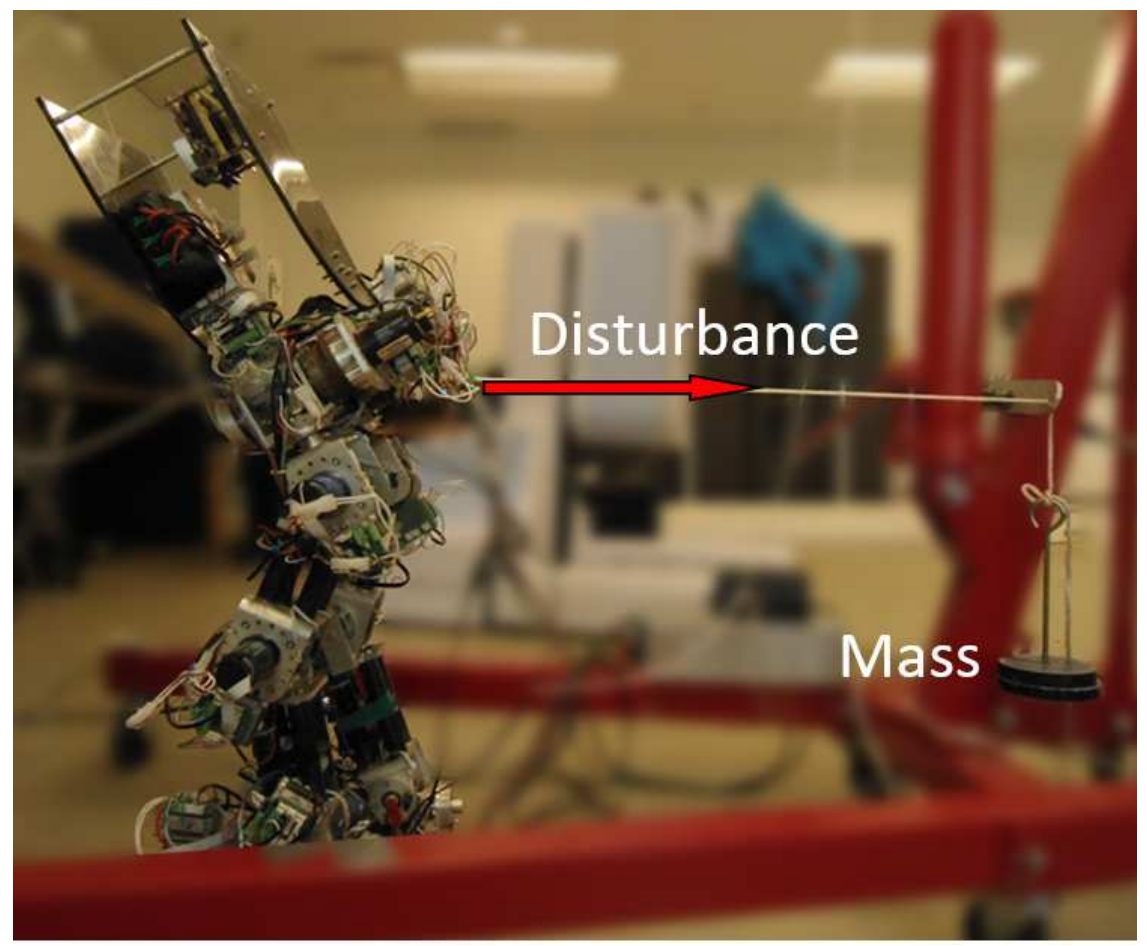

Figure 5.2: Method for calibrating lateral GRF estimation. A known mass is suspended generating a disturbance of a known magnitude acting on ABL-BI.

It is assumed the total GRF should be equal and opposite to the applied disturbance. This calibration resulted in the estimator parameters listed in Table 5.1. The calibration curves can be found in Appendix B.

\subsection{BBLC Experiments on ABL-BI and Analysis}

This section presents the experimental results for the four cases examined in simulation: (1) a walking experiment similar to the one in Section 5.2.1. (2) push disturbance experiment in Section 5.2.2, (3) step disturbance in Section 5.2.3 and (4) walking up a slope in Section 5.2.4. In all cases, any disturbance that is applied is not known by the BBLC. Hence, the key questions examined by these experiments are: (1) will the BBLC identify the disturbance applied by using the Q-table learnt in simulation, (2) will the BBLC activate the appropriate balancing strategy and (3) will the balancing strategy increase ABL-BI's robustness to disturbances? 


\subsubsection{Periodic Walking Experiment}

In the walking experiment, ABL-BI is placed on flat, level ground and no disturbances are applied to the robot. The walking gait is planned with the gait parameters listed in Table 5.2 which are equivalent to the gait parameters used in the simulation. The primary objective of this experiment is to examine if the robot settles into a balanced walking pattern using the BBLC architecture and if any balancing strategies are applied using the pre-learnt Q-table.

A successful walking gait was generated and data was collected for a total of 20 steps. The resulting motion of the robot is shown in Figure 5.3, where each phase of walking is shown for a complete cycle (including right and left foot steps). To examine if this gait is cyclic, and there is no divergence in the trajectory, the phase plane and Poincaré plots for the COM motion in the $\mathrm{X}, \mathrm{Y}$ and $\mathrm{Z}$ axes are shown in Figures 5.4 to 5.6. As in the simulation, the Poincaré points are taken at the state change between left foot swing and double stance right (walking state 6 to 1 ). The grouping of the Poincaré points is within $0.002 \mathrm{~m}$ for the COM position and $4.0 * 10^{-4} \mathrm{~m} / \mathrm{s}$ for the COM velocity. This indicates that the motion of the COM is repeatable between cycles. The cyclic nature of the gait is further confirmed in the phase plane plots shown in Figures 5.4(a), 5.5(a) and 5.6(a), where the trajectory between cycles are coincident. This result indicates that the generated walking gait should remain balanced indefinitely, as long as ABL-BI continues to walk on flat, level ground.

Table 5.2: Gait parameters used for walking experiment.

\begin{tabular}{|lr|}
\hline Gait Parameter & Value \\
\hline Step swing time & $3 \mathrm{~s}$ \\
Double stance time & $3 \mathrm{~s}$ \\
Step length & $0.10 \mathrm{~m}$ \\
\hline
\end{tabular}




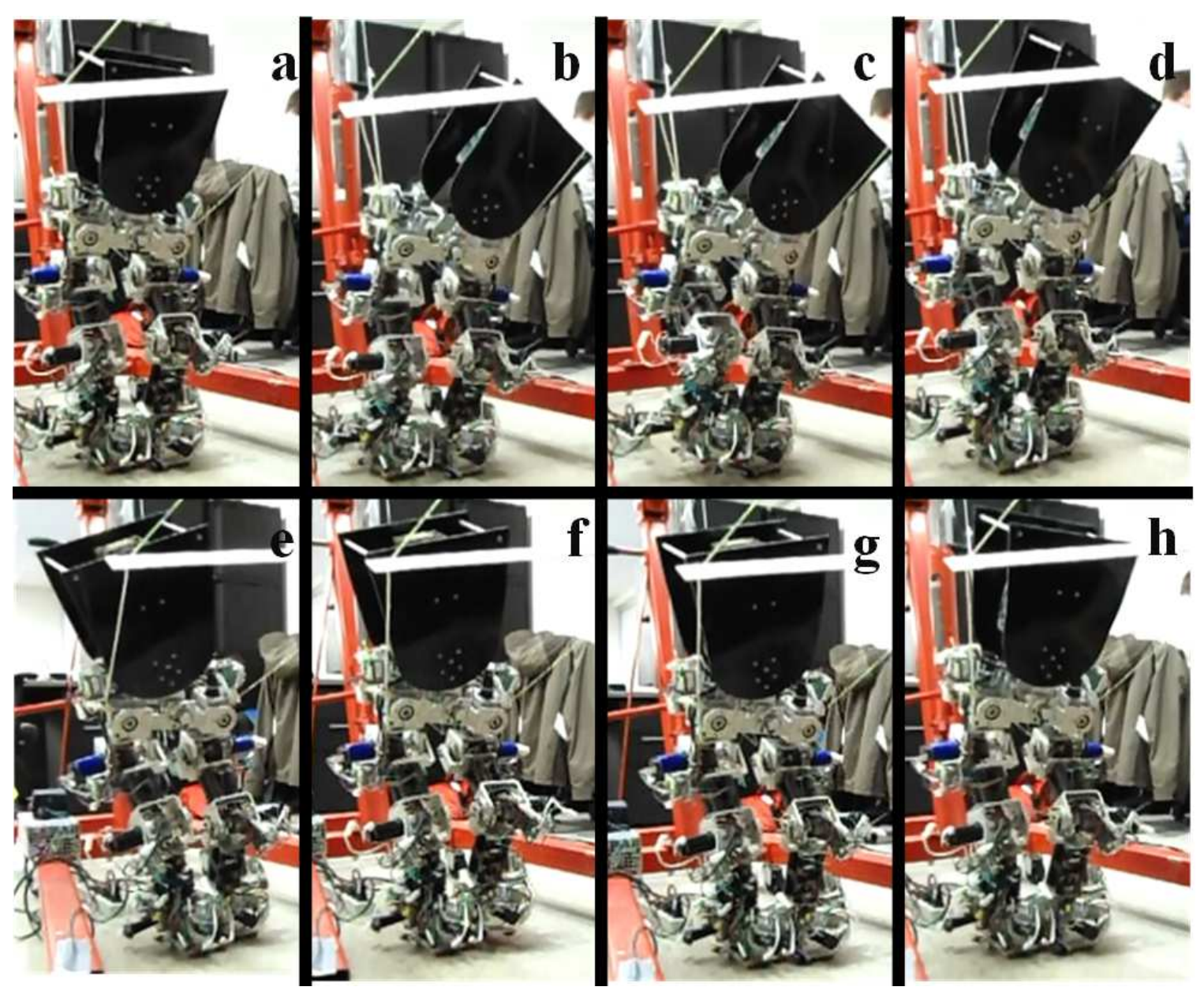

Figure 5.3: Resulting motion of ABL-BI during a balanced cyclic walk. Each frame corresponds to the following walking states: (a) DBL, (b) DBLS, (c) SSL, (d) DBR, (e) DBRS, (f) SSR, (g) DBL, (h) DBL.

The second item which can be examined in this experiment is if the BBLC applies any balancing strategies when the robot is undisturbed. A given balancing strategy will be activated by the BBLC if the system enters a state-space where $Q\left(\bar{s}_{t}, \vec{A}_{t}\right)<Q\left(\bar{s}_{t}, \vec{A}_{t}^{\prime}\right)$, where $\vec{A}_{t}^{\prime}$ is an alternative action vector and $\vec{A}_{t}=\left[\begin{array}{llllll}1 & 1 & 1 & 1 & 1 & 1\end{array}\right]$. Hence, the state vector $\bar{s}$, must be equivalent to a set of states that in simulation resulted in a fall, and has a negative Q-value. Although the simulation was designed to be analogous to ABL-BI, there will be differences in the system dynamics as well as unmodeled effects such as noise and 

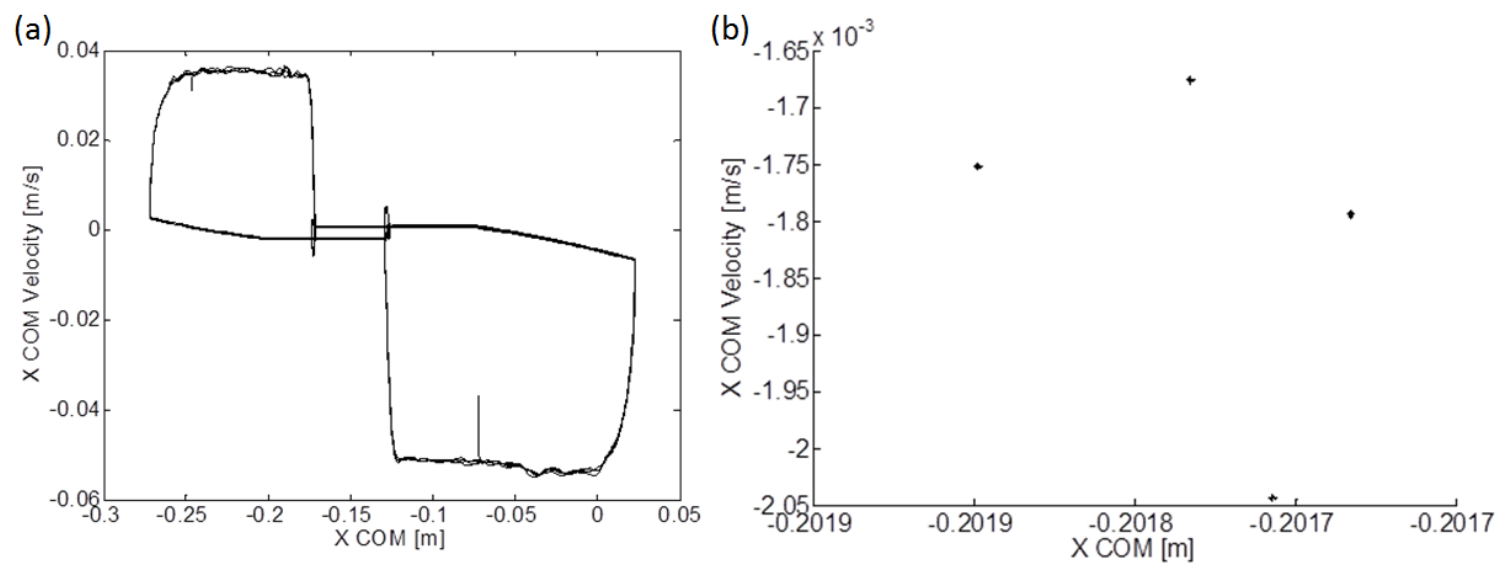

Figure 5.4: The phase plane of the X COM motion is shown in (a) and corresponding Poincaré map in (b) for 8 steps of the walking experiment.
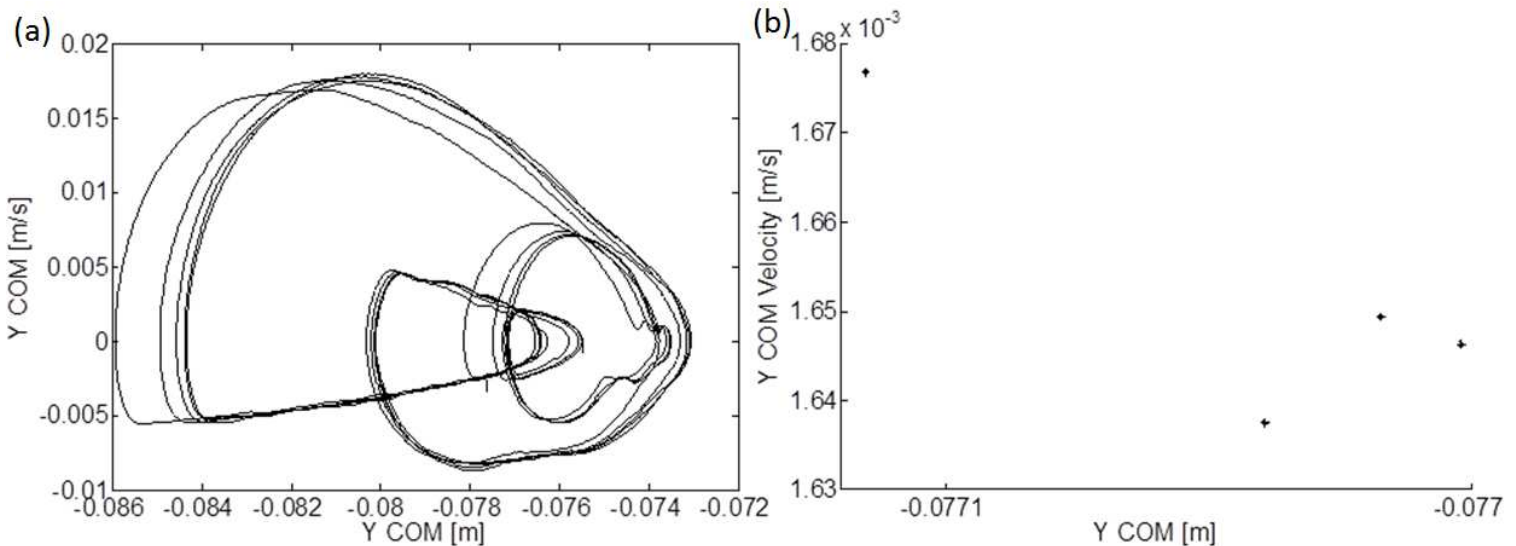

Figure 5.5: The phase plane of the Y COM motion is shown in (a) and corresponding Poincaré map in (b) for 8 steps of the walking experiment. 

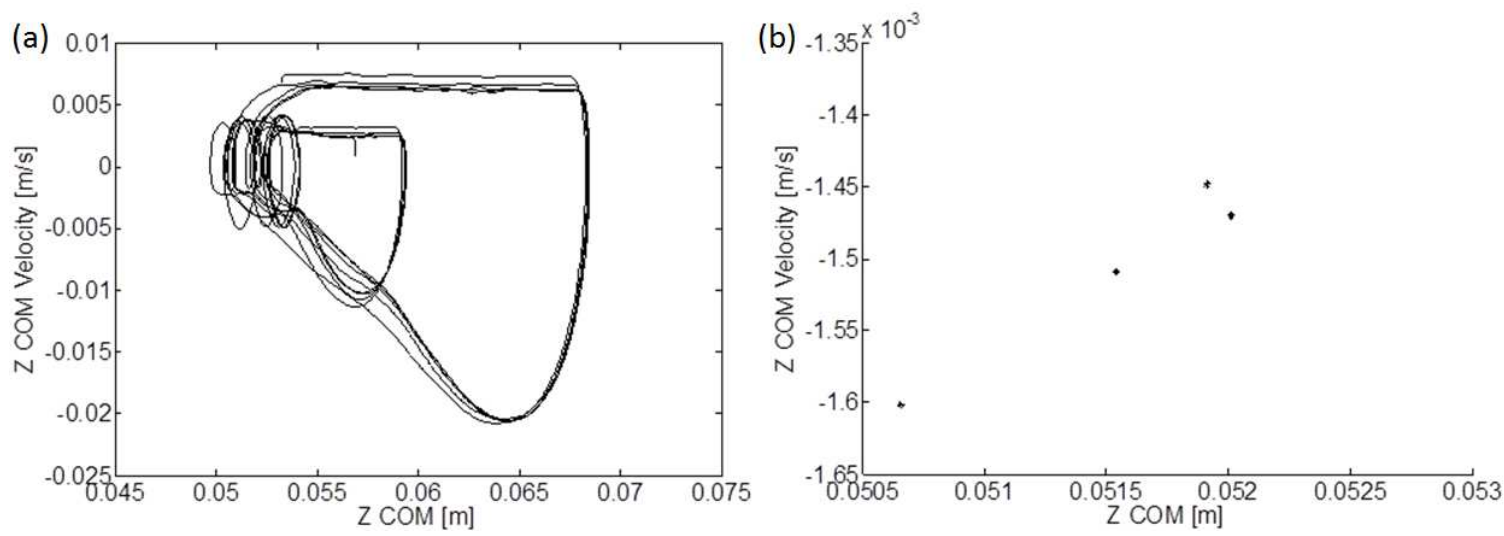

Figure 5.6: The phase plane of the Z COM motion is shown in (a) and corresponding Poincaré map in (b) for 8 steps of the walking experiment.

sensor performance. This may result in the measured state in the experiment to differ from the simulated state, and, as a consequence, the BBLC may activate a balancing strategy. For the walking experiment, this is not observed; the default action vector is applied throughout the walking motion. This indicates that during walking, the region spanned by the state vectors $\bar{s}$ in both the simulation and experiment are coincident.

\subsubsection{Push Disturbance Experiment}

In this test, a push-type disturbance is manually applied to the robot, when the robot is in single stance, as shown in Figure 5.7. This represents the worst case scenario, where the robot has a smaller support polygon. The magnitude of the force is not known, but estimated by the system as described in Section 5.1. This experiment diverges from the conditions in the push simulation experiment, where a pure constant force of known magnitude was applied to the robot. Given that the force is applied manually, there will be some variance in the force vector during application and the actual magnitude of the force is unknown.

The first objective of the experiment is to examine if the balancing strategy learnt in 


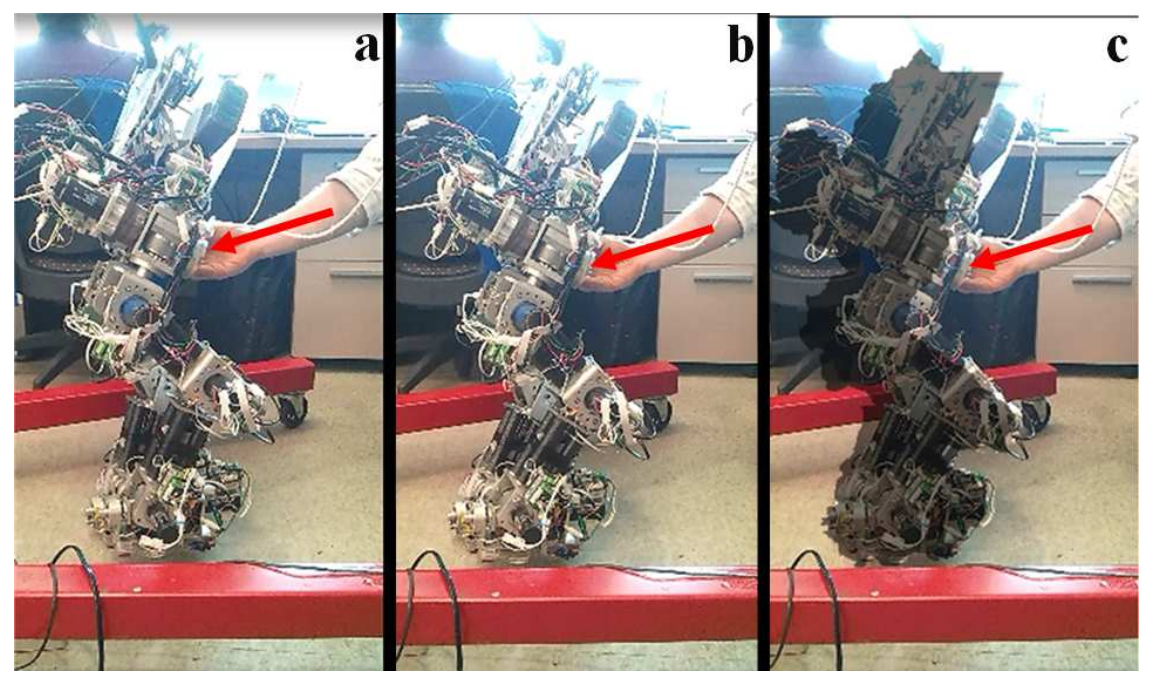

Figure 5.7: Resulting motion of ABL-BI when a push disturbance is applied to the pelvis during SSL. Frame (a) corresponds to the initial posture before the disturbance is applied, (b) the posture after the disturbance is applied and (c) a comparison of the intail and final postures, where the shaded area corresponds to the initial posture.

simulation will be activated to compensate for the push disturbance. As shown in Figure 5.8 , it can be seen that a balancing strategy is activated $0.52 \mathrm{~s}$ after the disturbance is applied. The strategy that is applied has an action vector of $\vec{A}=\left[\begin{array}{llllll}1 & 3 & 2 & 3 & 2 & 1\end{array}\right]^{T}$ which is the equivalent strategy that was learnt in simulation for a push-type disturbance. Activating this balancing strategy is a result of the robot entering a state where

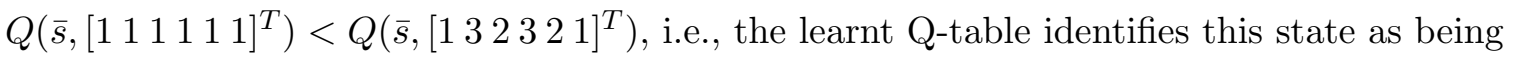
unbalanced if the default action vector is applied. At the time of the strategy activation $\bar{s}=\left[\begin{array}{lllll}2 & 5 & 6 & 3 & 5\end{array}\right]^{T}$ in simulation and $\bar{s}=\left[\begin{array}{lllll}2 & 5 & 5 & 3 & 5\end{array}\right]^{T}$ in the experiment. A comparison with the state vector from a simulated push disturbance case is also shown in Table 5.3 . It can be seen that the state indices are all equivalent except in the force index for Z GRF. This is primarily a result of the different load conditions that are applied to the system. This indicates that despite the difference in the experimental conditions, the robot enters a state-space where the controller has learnt to activate a balancing strategy. Additionally, the reduced state-space $\bar{s}$ appears to be robust to variances in the dynamics and kinematic parameters, given that for both simulation and experiment the robot enters a similar state-space. 
Table 5.3: Comparison of state vector initiating balancing strategy between experiment and simulation for the push disturbance experiment.

\begin{tabular}{|lrrr|}
\hline State & $\begin{array}{r}\text { State Value } \\
\text { (Experiment) }\end{array}$ & $\begin{array}{r}\text { State Index } \\
\text { (Experiment) }\end{array}$ & $\begin{array}{r}\text { State Index } \\
\text { (Simulation) }\end{array}$ \\
\hline $\begin{array}{l}\text { Stability } \\
\text { Margin }\end{array}$ & $0.0145 \mathrm{~m}$ & 2 & 2 \\
X GRF & $-2.6 \mathrm{~N}$ & 5 & 5 \\
Z GRF & $0.0 \mathrm{~N}$ & 6 & 5 \\
Walking State & 3 & 3 & 3 \\
Slope & $0^{\circ}$ & 5 & 5 \\
Estimate & & & \\
\hline
\end{tabular}

The equivalence between the simulated and experimental state vectors is a result of the design of the reduced state-space vector. The coarse discretization acts to filter out variations in states that are smaller than the discretization range. For example, the GRF values are not equivalent comparing Figures 5.9 (b) and 5.10 (b) as well as Figures 5.11 (b) and 5.12 (b). However, given that the discretization is coarse (4 N resolution for $\mathrm{X}$ GRF and $6 \mathrm{~N}$ resolution for $\mathrm{Z}$ GRF), force values can result in an equivalent state index, e.g., for $\mathrm{X}$ GRF values between $-4 \mathrm{~N}$ and $0 \mathrm{~N}$ the index will be 5 . The second factor is the dimensionality of the reduced state-space where $\bar{s} \in \mathbb{R}^{5}$. This reduces the size of the examined state-space to five key variables which are not as sensitive to the dynamics of the system, such as joint inertias, which are not equivalent between the simulation and experimental platform. Rather, the state-space is composed primarily of model independent variables, which are consistent between the experiment and simulation; thus explaining why the same balancing strategy is activated in both simulation and experiment.

The second aspect to examine in this experiment is if the balancing strategy defined by

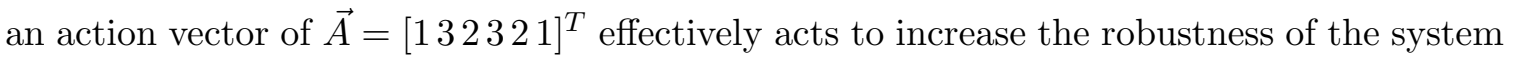
to push-type disturbances, as demonstrated in the simulation. This strategy primarily consists of applying the CMP planning controller for both the $\mathrm{X}$ and $\mathrm{Z} \mathrm{COM}$ motions and 


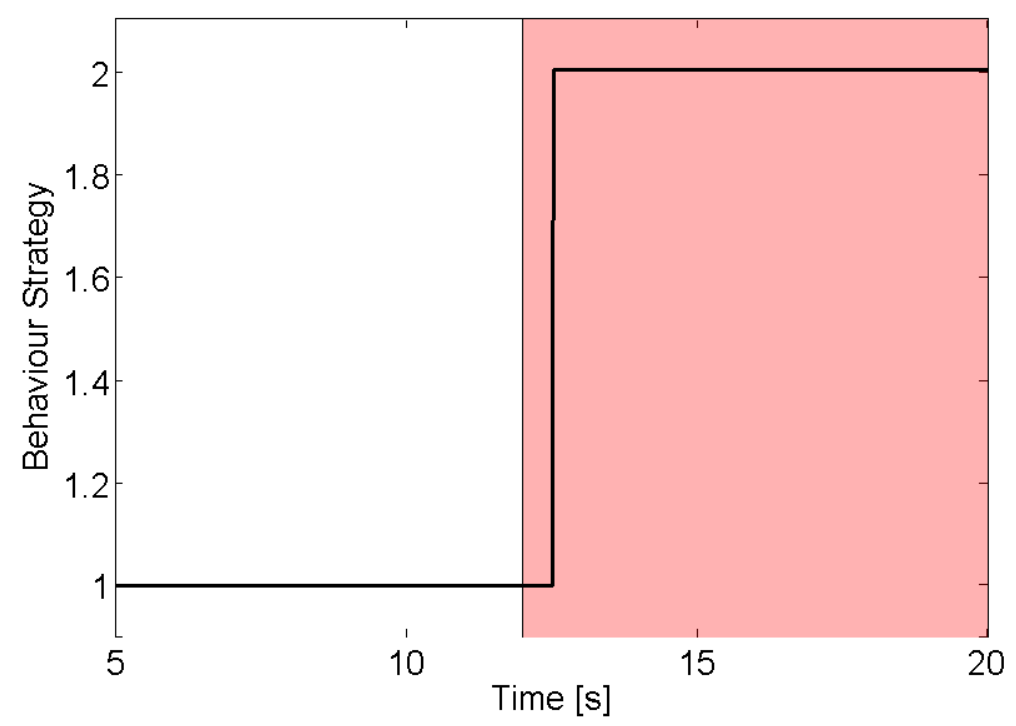

Figure 5.8: The balancing strategy being used in the BBLC controller where strategy 2 corresponds to an activation vector of $\vec{A}=\left[\begin{array}{llllll}1 & 3 & 2 & 3 & 2 & 1\end{array}\right]^{T}$. The strategy is activated at 12.52 $\mathrm{s}$ and the magnitude of the disturbance is $15 \mathrm{~N}$ in the $\mathrm{X}$ axis and $23 \mathrm{~N}$ in the $\mathrm{Z}$ axis (red area corresponds to the period when the disturbance is applied).

(a)

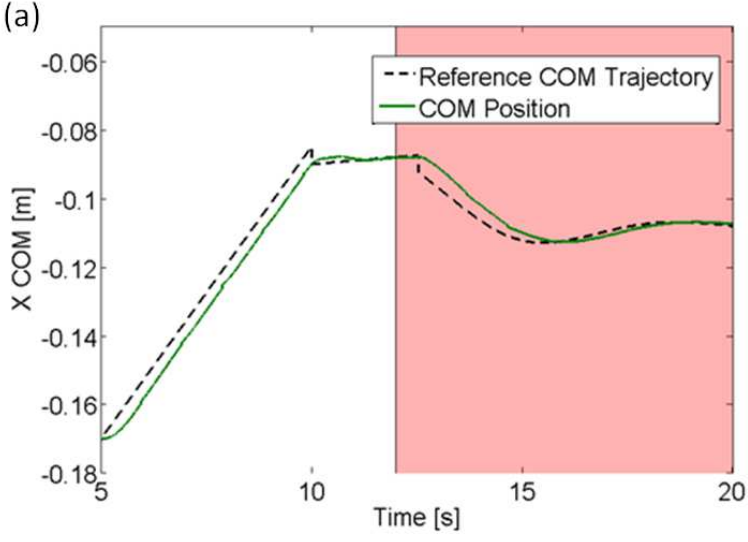

(b)

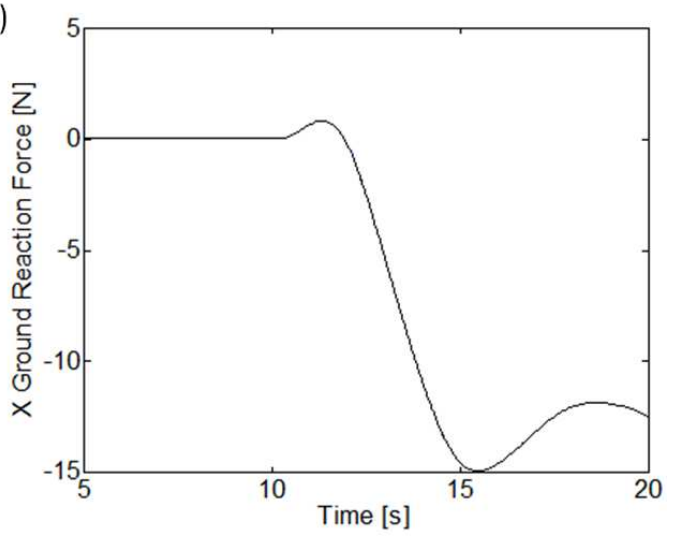

Figure 5.9: As a result of the push disturbance (red area corresponds to the period when the disturbance is applied) the CMP behaviour is activated for planning the COM motion. The resulting motion for X COM is shown in (a) and the estimated GRF in the X axis in (b). 
(a)

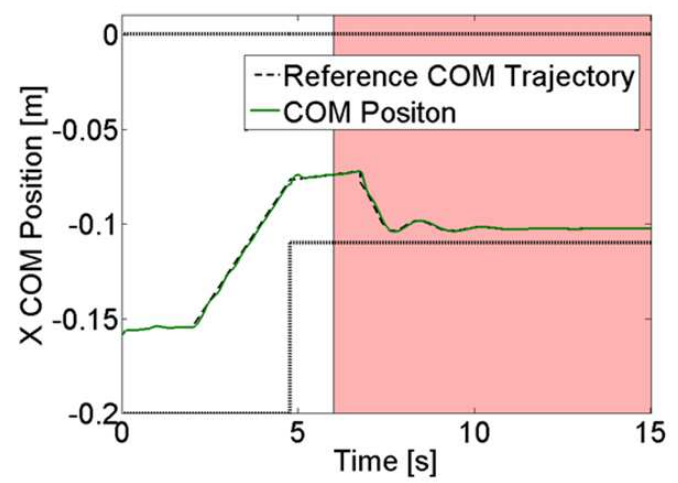

(b)

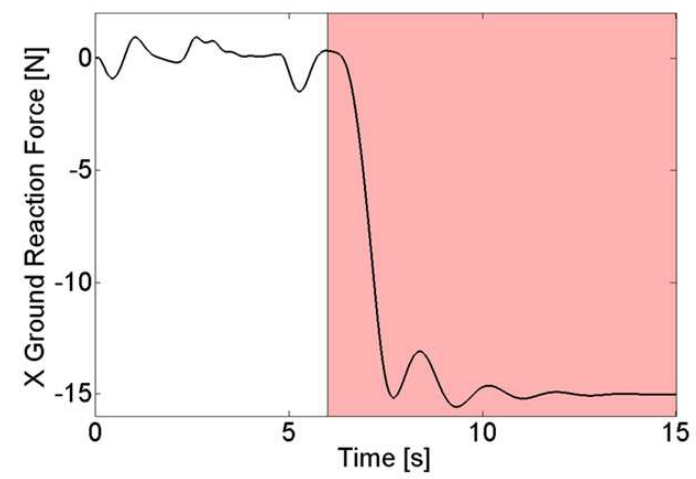

Figure 5.10: As a result of the push-type disturbance (red area corresponds to the period when the disturbance is applied) the CMP behaviour is activated for planning the COM motion. The resulting motion for X COM is shown in (a) where the dotted boundary denotes the support polygon and the measured GRF in the X axis in (b).

(a)

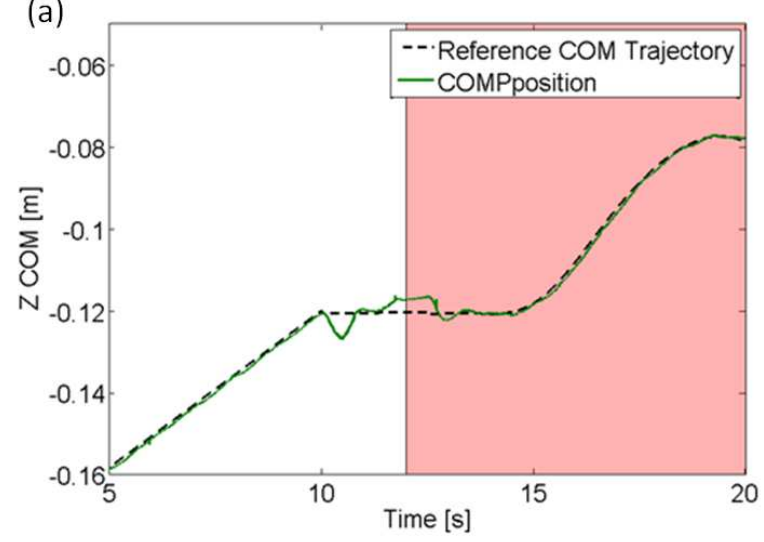

(b)

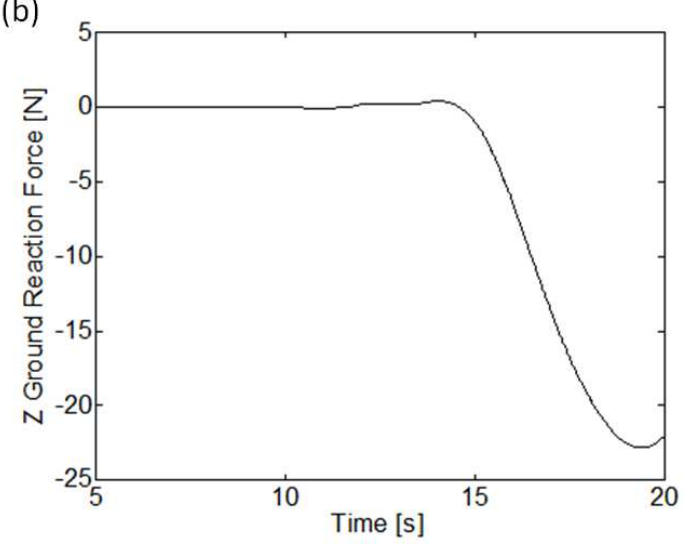

Figure 5.11: As a result of the push disturbance (red area corresponds to the period when the disturbance is applied) the CMP behaviour is activated for planning the COM motion. The resulting motion for $\mathrm{Z} \mathrm{COM}$ is shown in (a) and the estimated GRF in the $\mathrm{Z}$ axis in (b). 
(a)

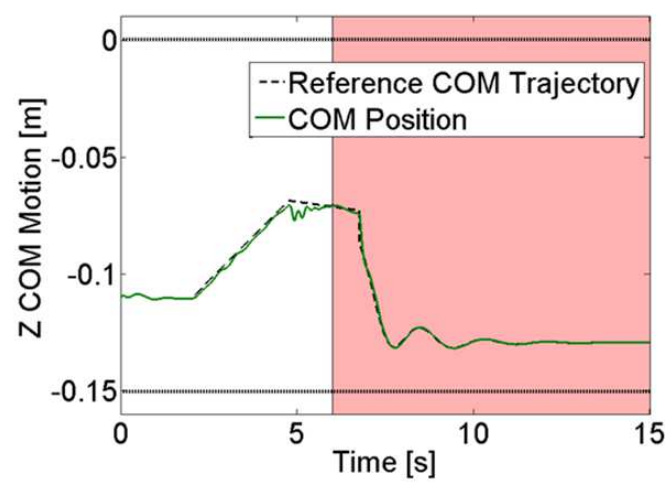

(b)

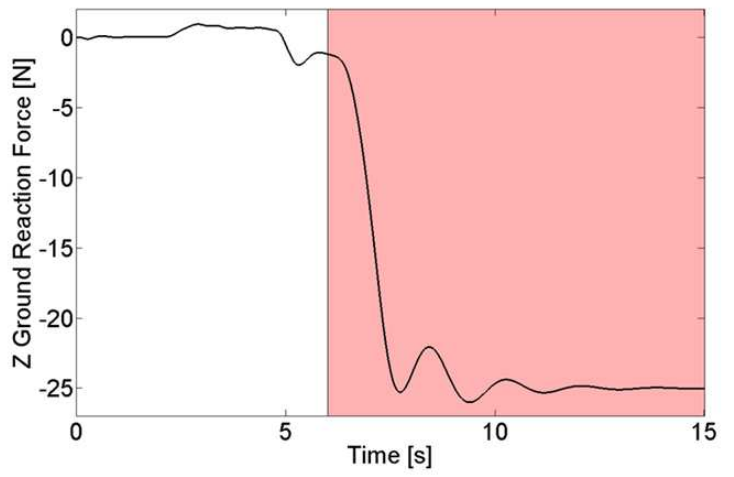

Figure 5.12: As a result of the push-type disturbance (red area corresponds to the period when the disturbance is applied) the CMP behaviour is activated for planning the COM motion. The resulting motion for Z COM is shown in (a) where the dotted boundary denotes the support polygon and the measured GRF in the $\mathrm{Z}$ axis in (b).

excluding swing leg control. For a disturbance of $15 \mathrm{~N}$ along the $\mathrm{X}$ axis and $23 \mathrm{~N}$ along the $\mathrm{Z}$ axis, the resulting change in the COM trajectory from applying this balancing strategy is shown in Figures 5.9 (a) and 5.11 (a) and the estimated GRFs are shown in Figures 5.9 (b) and 5.11 (b). These figures show that the COM motion planning is responding to the disturbance being applied, similarly to what was observed in simulation. This is reflected in the motion of ABL-BI as shown in Figure 5.7, where the change in posture as a result of applying the disturbance is shown relative to the initial posture of the robot, before the disturbance was applied. Qualitatively, ABL-BI moves forward against the direction of the force being applied.

The effect on the robustness to push-type disturbances during single stance is illustrated in Figure 5.13, where the data points correspond to disturbance magnitudes for which ABL-BI was able to maintain balance. The area delimited by the dashed box corresponds to the region for which not applying the balancing strategy (i.e., using $\vec{A}=\left[\begin{array}{llllll}1 & 1 & 1 & 1 & 1 & 1\end{array}\right]$ ) was able to maintain balance. This figure shows that there is a clear increase in the robustness of the system to external push disturbances, with a $10 \mathrm{~N}$ increase in $\mathrm{X}$ and $25 \mathrm{~N}$ increase in $\mathrm{Z}$. 


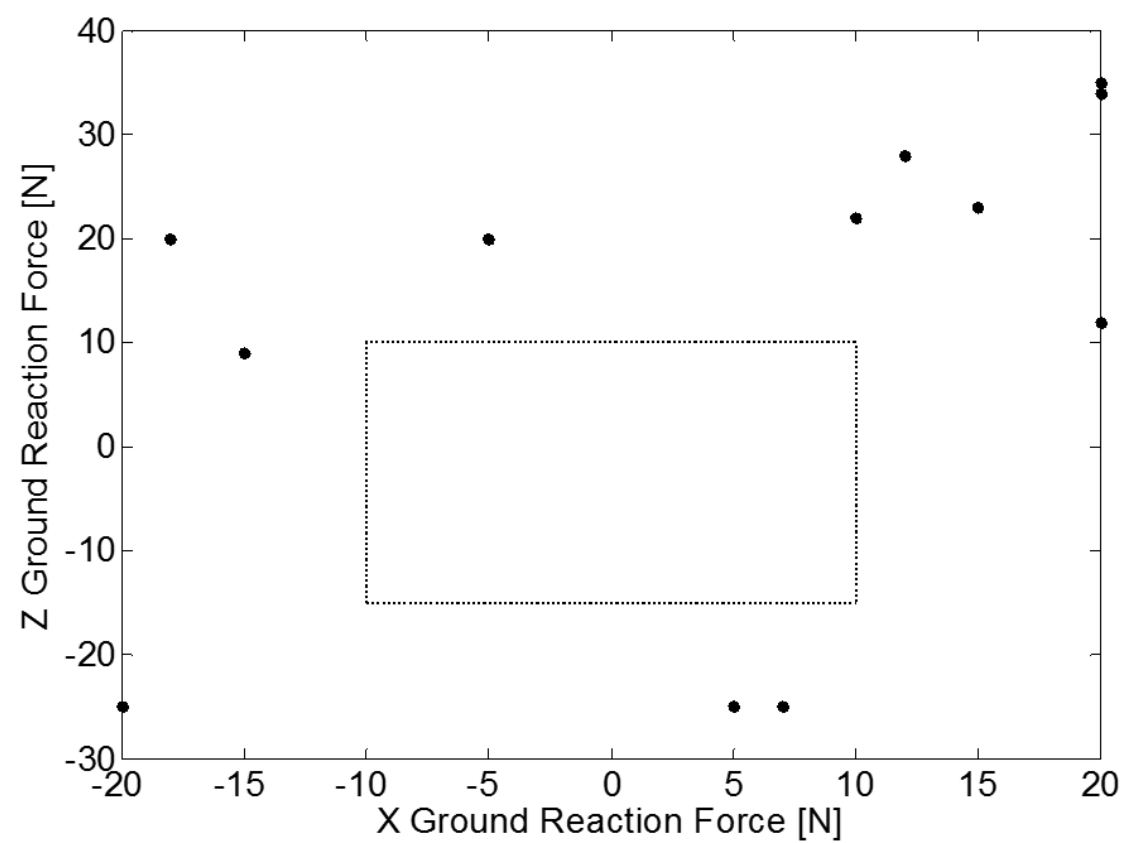

Figure 5.13: The set of different push disturbance tests resulting in a balanced posture as a function of the magnitude of the disturbance. The dotted box corresponds to the boundary within which the robot remains balanced while applying the default action vector.

In summary the push disturbance experiment showed that the Q-table learnt in simulation could be transferred to ABL-BI and results in the same balancing strategy being applied to compensate for the push disturbance. Also, the experiment demonstrated that the balancing strategy resulted in increased robustness to push disturbances.

\subsubsection{Step Disturbance Experiment}

In this experiment a $0.01 \mathrm{~m}$ step is placed under one foot during walking. Initially the robot begins at rest with no step under either foot and walks using the gait parameters listed in Table 5.2 and with no a priori knowledge of the disturbance. The step is introduced by placing a plate under the right foot during SSL as seen in Figure 5.14. The plate is then moved forward as the robot continues to walk such that the right foot always lands on the plate. Similarly to the push experiment, the objective of this experiment is to examine if the BBLC will identify the step disturbance using the learnt Q-table from simulation, activate the same balancing strategy and if this strategy will be effective in maintaining balance. 
The resulting motion of ABL-BI with a step placed under the right foot is shown in Figure 4.15, where all phases of walking for one cycle are shown. In this experiment, ABL-BI is able to remain balanced while walking on the step, which is not the case when only the default action vector is applied. This is the result of applying the balancing strategy that was learnt in simulation and which corresponds to an action vector of $\vec{A}=\left[\begin{array}{lllll}1 & 1 & 2 & 3 & 2\end{array}\right]^{T}$, with the corresponding strategy activation for two cycles of walking shown in Figure 5.15. As in the simulation experiment, this figure shows that the balancing strategy is activated when ABL-BI is in SSR, i.e., when the robot is trying to remain balanced on the right foot. The state vector when the strategy is initially activated is listed in Table 5.4 with a comparison to the corresponding state vector from simulation. For this experiment, the state vectors are exactly equivalent between the simulated and experimental case, as seen in Table 5.4 .

Table 5.4: Comparison of the state vector for which a balancing strategy is activated in the simulated and experimental step walking experiments.

\begin{tabular}{|lrrr|}
\hline State & $\begin{array}{r}\text { State Value } \\
\text { (Experiment) }\end{array}$ & $\begin{array}{r}\text { State Index } \\
\text { (Experiment) }\end{array}$ & $\begin{array}{r}\text { Sate Index } \\
\text { (Simulation) }\end{array}$ \\
\hline $\begin{array}{l}\text { Stability } \\
\text { Margin }\end{array}$ & $0.008 \mathrm{~m}$ & 1 & 1 \\
X GRF & $5 \mathrm{~N}$ & 7 & 7 \\
Z GRF & $5.8 \mathrm{~N}$ & 6 & 6 \\
Walking State & 6 & 6 & 6 \\
Slope & $0^{\circ}$ & 5 & 5 \\
Estimate & & & \\
\hline
\end{tabular}

As with the walking experiment, the cycle stability of the walking motion can be evaluated by analyzing the phase plane trajectory and Poincaré map, shown in Figure 5.16 for the X COM motion. The state change used to generate the Poincaré map is again the state change between SSL and DS (walking state 6 to 1). The Poincaré points are clusters with $0.0014 \mathrm{~m}$ for the COM position and $0.002 \mathrm{~m} / \mathrm{s}$ for the COM velocity. This indicates the 


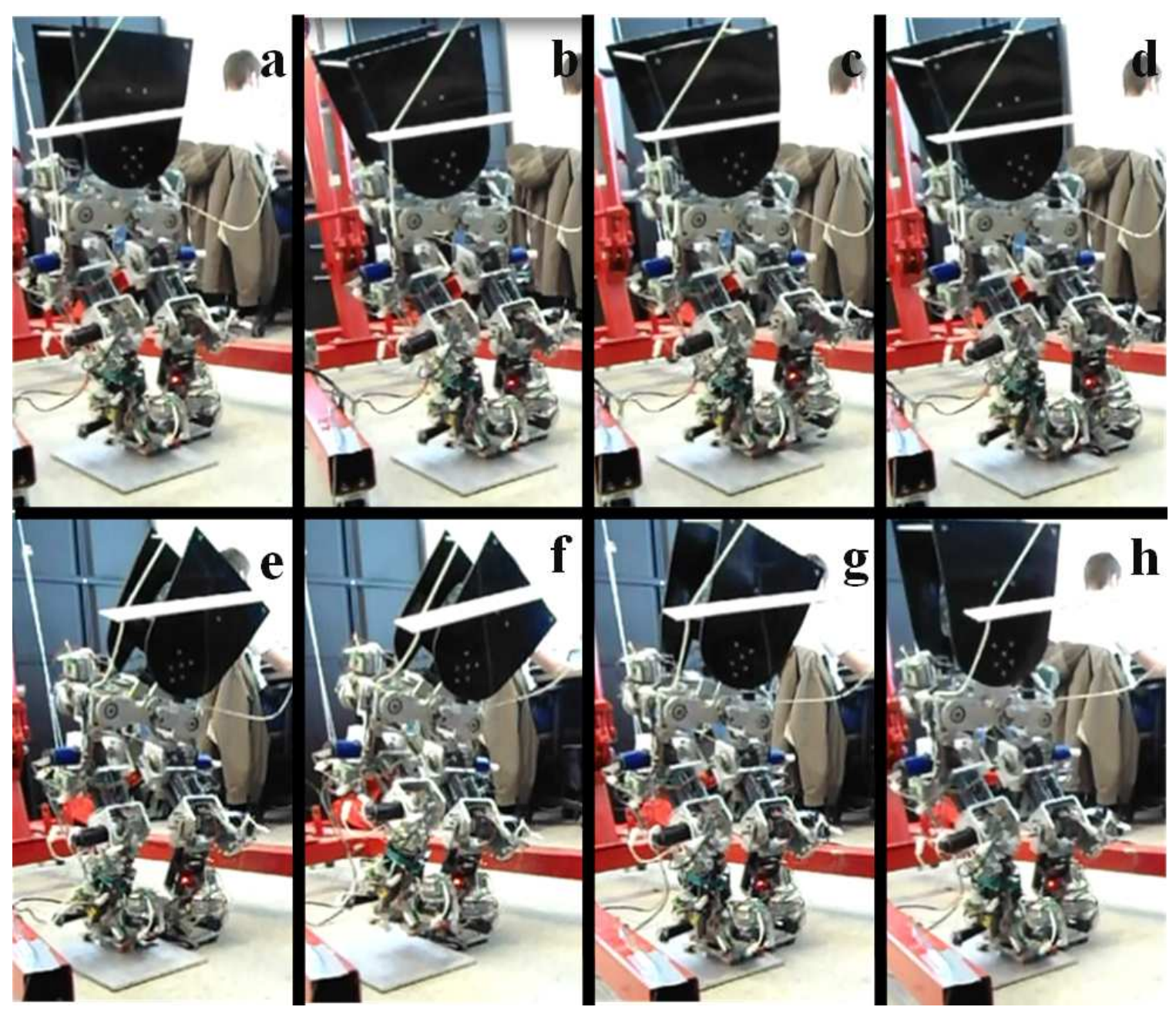

Figure 5.14: Resulting motion of ABL-BI during the step walk test. Each frame corresponds to the following walking states: (a) DBR, (b) DBRS, (c) SSR, (d) DBL, (e) DBLS, (f) SSL, (g) DBR, (h) DBR. 
(a)

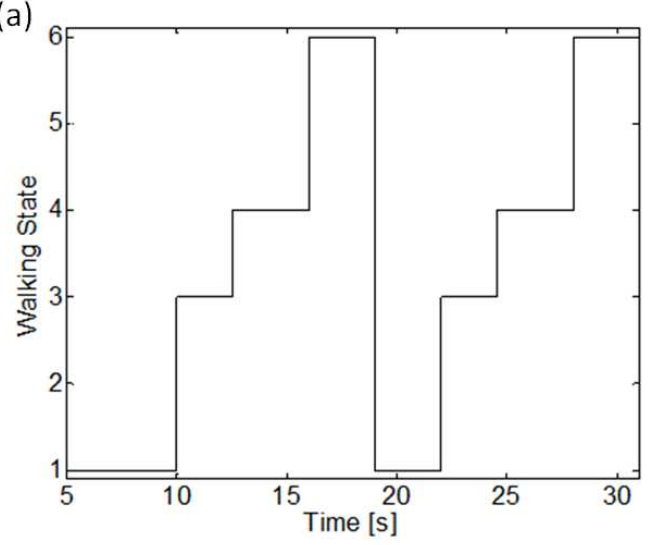

(b)

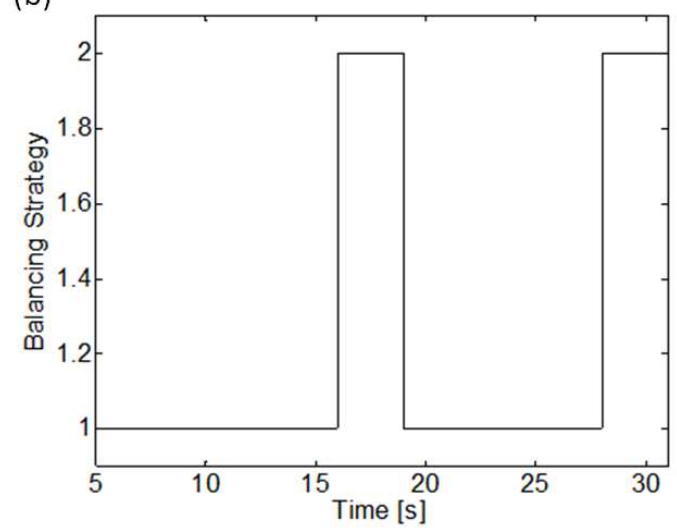

Figure 5.15: Activation of the balancing strategy when walking with a $1 \mathrm{~cm}$ step under the right foot in (b). The balancing strategy activation coincides with the robot being in single stance on the right foot (walking state 6) where the walking state is shown in (a).
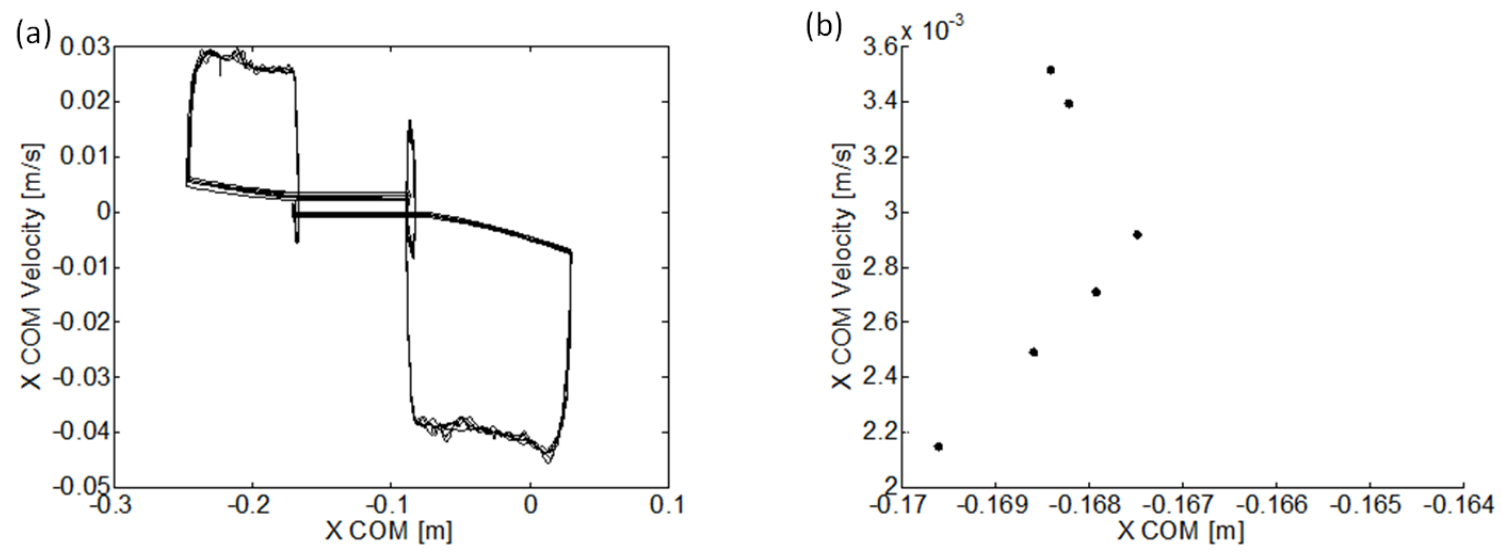

Figure 5.16: The phase plane of the X COM motion is shown in (a) and corresponding Poincaré map in (b) for 12 steps of the step walking experiment. 
cycle trajectory is repetitive and consistent which is also shown in the phase plane trajectory in Figure 5.16 (a), where it can be seen the trajectories in each cycle are coincident. Overall, this result indicates that the BBLC enters a state-space which was learnt to be unbalanced and applies the correct balancing strategy, resulting in a new stable walking cycle.

\subsubsection{Slope Disturbance Experiment}

The last experiment consists of placing a slope in ABL-BI's path. The robot initially starts on flat ground and begins to walk with the gait parameters listed in Table 5.2 with no a priori knowledge of the disturbance. From the first step, the robot beings walking up a ramp with a $5^{\circ}$ slope as shown in Figure 5.17. As with previous experiments, the objective is to examine if the BBLC will be able to identify the disturbance, activate the corresponding balancing strategy, learnt in simulation, and maintain balance while being exposed to the disturbance. Figure 5.18 shows the resulting motion for a complete cycle of walking as ABL-BI continues to walk up the slope.

As in the simulation results, the BBLC activates the balancing strategy with an action vector of $\vec{A}=\left[\begin{array}{llllll}2 & 2 & 1 & 3 & 1 & 1\end{array}\right]^{T}$ during the single stance phases of the walking motion, as shown in Figure 5.19. Note that without this balancing strategy applied, ABL-BI tips over when trying to walk up the slope. The corresponding state vectors resulting in the activation of the balancing strategy are listed in Table 5.5. These results show that as with the push experiment, the state vectors for both the experiment and simulation are similar, but not equivalent. However, given that the balancing strategy was activated, this indicates the state vector lies within the state-space where the balancing strategy was learnt to be effective. This is what results in the same balancing strategy being applied in both cases.

As in the simulation, applying the balancing strategy with $\vec{A}=[221311]^{T}$ results in the robot adapting to the slope by estimating the slope angle, adjusting the foot angle to the slope when the swing leg lands, as well as activating CMP planning control during single 


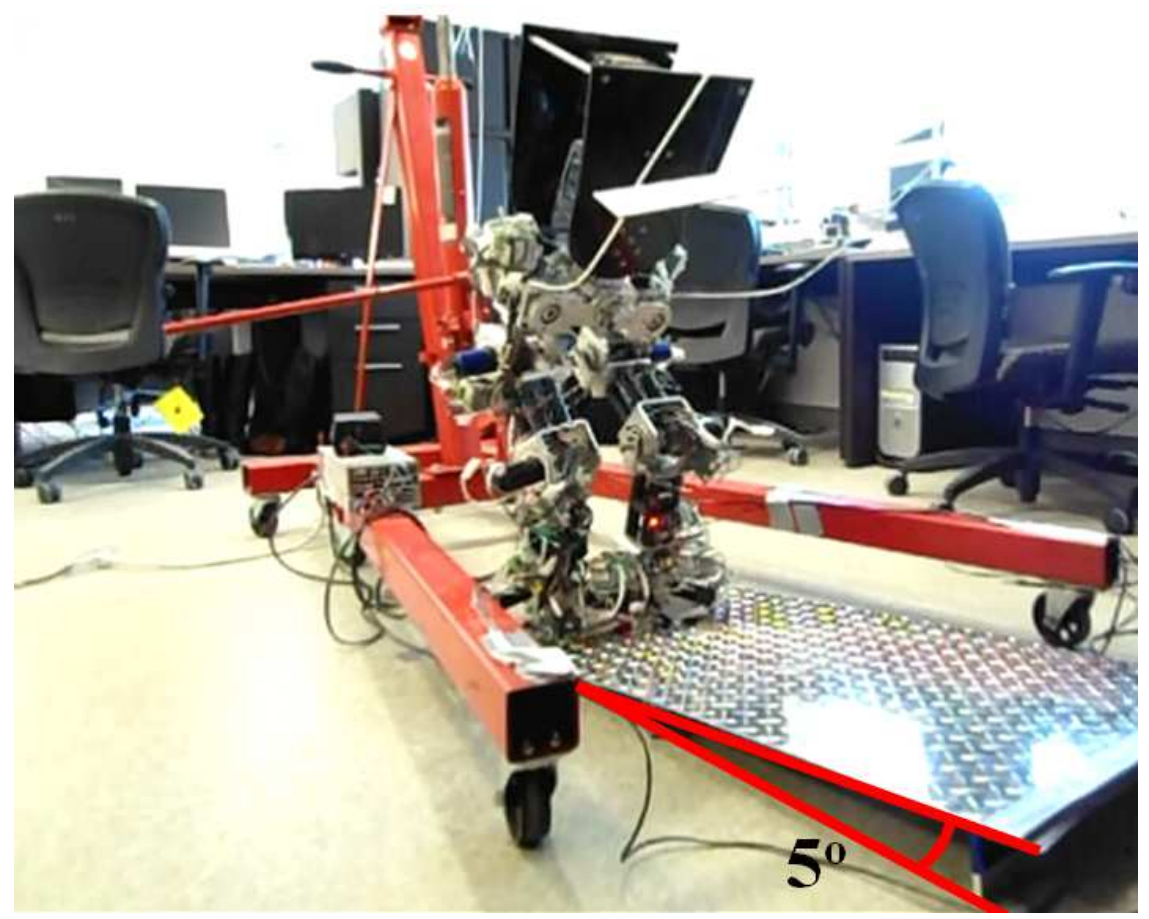

Figure 5.17: The $5^{\circ}$ slope along which ABL-BI walks during the slope experiment.

Table 5.5: Comparison of the state vectors for which a balancing strategy is activated in the simulated and experimental slope walking experiments.

\begin{tabular}{|lrrr|}
\hline State & $\begin{array}{r}\text { State Value } \\
\text { (Experiment) }\end{array}$ & $\begin{array}{r}\text { State Index } \\
\text { (Experiment) }\end{array}$ & $\begin{array}{r}\text { Sate Index } \\
\text { (Simulation) }\end{array}$ \\
\hline Stability & $0.044 \mathrm{~m}$ & 5 & 2 \\
Margin & & & 3 \\
X GRF & $-11.1 \mathrm{~N}$ & 3 & 6 \\
Z GRF Force & $5.7 \mathrm{~N}$ & 6 & 3 \\
Walking State & 3 & 3 & 10 \\
Slope & $5^{\circ}$ & 10 & \\
Estimate & & & \\
\hline
\end{tabular}




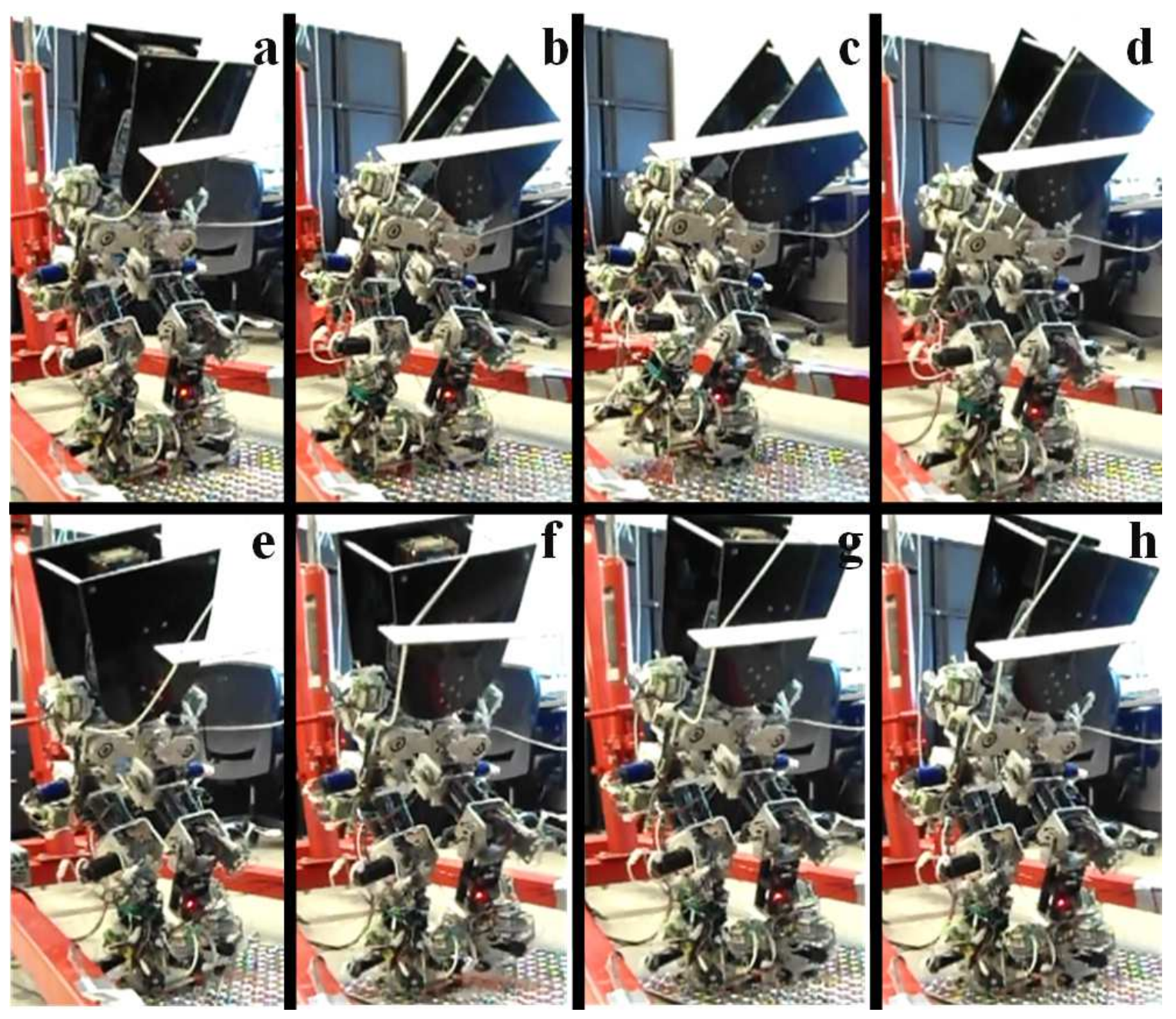

Figure 5.18: Resulting motion of ABL-BI during the step walk test. Each frame corresponds to the following walking states: (a) DBL, (b) DBLS, (c) SSL, (d) DBR, (e) DBRS, (f) SSR, (g) DBL, (h) DBL. 
(a)

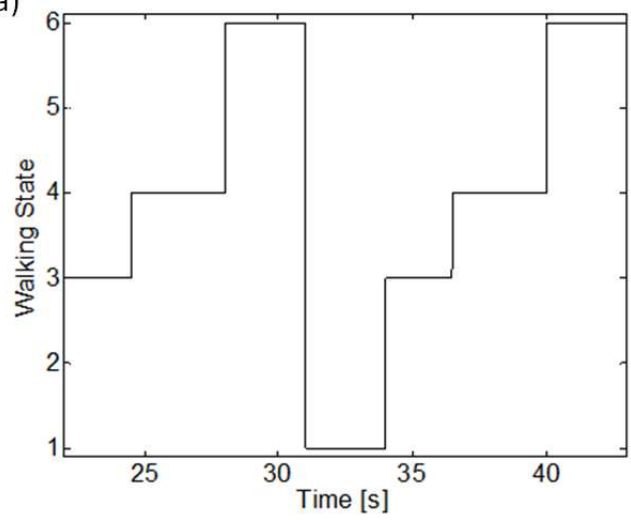

(b)

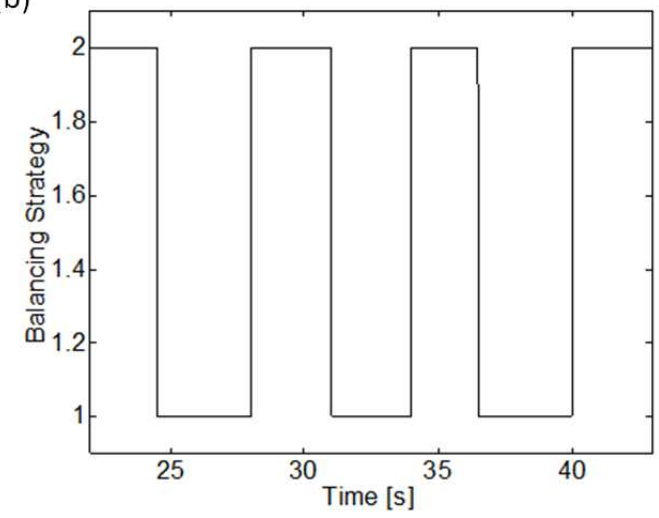

Figure 5.19: Balancing strategy activation when walking on a $5^{\circ}$ slope shown in (b). The balancing strategy activation coincides with single stance for both the right and left foot, where the walking state is shown in (a).
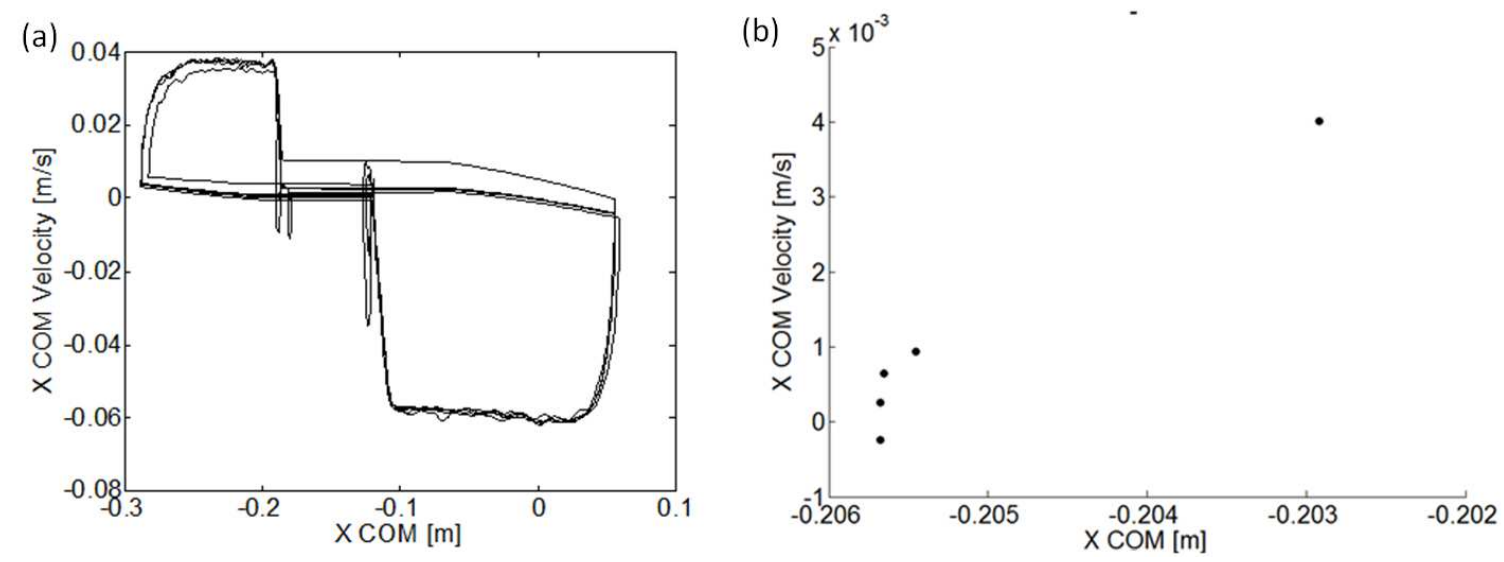

Figure 5.20: The phase plane of the X COM motion is shown in (a) and corresponding Poincaré map in (b) for a 10 steps of the slope walking experiment. 
stance. As with the Step and Walking experiments, the cycle stability of the generated walking motion can be examined through a phase plane and Poincaré map analysis, shown in Figure 5.20, where the Poincaré point is taken at the state transition change between SSR and DBL (walking states 6 to 1). As with previous cases, the clustering of the Poincaré points to within $0.003 \mathrm{~m}$ along the COM position and $0.005 \mathrm{~m} / \mathrm{s}$ along the COM velocity, indicates the cycle is repetitive. This is reflected in the coincident phase plane trajectories in 5.20 (a). This result indicates that as long as the conditions of the disturbance do not change, the walking motion will remain balanced and stable.

\subsection{Chapter Summary}

In this chapter the experimental results from applying the BBLC on the ABL-BI were presented. Four different cases were examined:

1. Cyclic walking experiment to examine if a balanced cyclic walking gait could be generated

2. Push disturbance experiment where ABL-BI was exposed to a push-type disturbance

3. Step experiment where a step was placed under one of the feet of ABL-BI while it was walking

4. Slope experiment where a $5^{\circ}$ slope was placed in ABL-BI's path during walking

The results from the experiments listed above clearly show that the balancing strategies that were activated in simulation and the experiments were equivalent. This was the result of the system entering the same reduced state-space when the biped was exposed to a similar disturbance, either in simulation or experiment. In other words, the Q-table that was learnt in simulation was indeed applicable to the experimental environment as well, without any additional learning. The learnt Q-table was also transferrable despite modelling errors and variations in sensor data between the experiment and simulation. This is a powerful feature, given that performing the learning of the Q-table is much easier 
and less costly in simulation. The robot can fall without causing damage, disturbance cases can be easily applied and many iterations can be simulated in a shorter time frame, as compared with on-line learning. This also contrasts to other approaches [76, 87, 90, 138] in bipedal learning, where tuning of gait parameters, or CPGs through learning were typically done on the experimental platform.

In each experimental case, the robot was able to react to the applied disturbance and maintain balance. This provides a strong answer to the final research question, in that the balancing strategies were able to compensate for the disturbances being applied. Additionally, this result validates the core novel aspect of the BBLC controller: new balancing strategies emerge from a subset of simpler balancing behaviours to compensate for unknown disturbances. These results present the first validation of such a BBC architecture for bipedal locomotion, where previously, emergent behaviour-based controllers were primarily applied in mobile robot navigation $44,45,118,139,140$. 


\section{Chapter 6}

\section{Stability Analysis of Behaviour Strategies Using the Linear Inverted Pendulum Model}

In previous chapters, the structure of the BBLC was presented along with simulation and experimental results. These results showed that the BBLC can effectively generate new balancing strategies to unknown disturbances by combining a set of balancing behaviours. However, given that these strategies emerge from applying a reinforcement learning algorithm, the underlying reasoning as to why these strategies succeed is not immediately obvious. The objective of this chapter is to further understand why these new strategies succeed. This is done by applying a stability analysis of the Linear Inverted Pendulum Model (LIPM) to understand how the stability of the biped is affected by applying different balancing behaviours. The push disturbance case is then analyzed in further detail using this framework.

\subsection{LIPM Stability Analysis}

As introduced in Chapter 1, the LIPM has been used extensively to plan stable walking trajectories for bipedal walking [62. The resulting simplified system is linear and can be analyzed to establish constraints for balanced walking $39,41,43,141$. Using this framework the effect of different balancing behaviours can be examined analytically to understand how 
they affect balance and why the BBLC uses them in emergent balancing strategies. More specifically, the behaviour applied in the push compensation strategy $\left(\vec{A}=\left[\begin{array}{lllll}1 & 3 & 2 & 3 & 2\end{array}\right]^{T}\right)$ will be examined. In this strategy, the swing leg motion planning is excluded from the task-priority control and CMP based planning is used to plan the X and Z COM trajectories.

The basic formulation of the dynamic equations of the LIPM presented in (1.1) and $(1.2)$, where the moment balance of the system is

$$
\begin{aligned}
& F_{x}=\frac{F_{y}}{y_{C O M}}\left(x_{C O M}-x_{C O P}\right), \\
& F_{z}=\frac{F_{y}}{y_{C O M}}\left(z_{C O M}-z_{C O P}\right) .
\end{aligned}
$$

The model can be further simplified by assuming that

$$
F_{x}=m \ddot{x}_{C O M},
$$

and

$$
F_{y}=m g
$$

where $m$ is the total mass of the robot and $g$ is gravitational acceleration. This results in the simplified equation

$$
\ddot{x}_{C O M}=\frac{g}{y_{C O M}}\left(x_{C O M}-x_{C O P}\right) .
$$

Note that from this point, the derivation will be presented only for the X Axis, while it can be similarly reproduced for the $\mathrm{Z}$ axis. The stability of this system can be examined by applying the ZMP balance constraint where the COP must lie within the support polygon of the biped, $-x_{C O P}^{\max }<x_{C O P}<x_{C O P}^{\max } 102$. For this derivation it is assumed that the reference frame is placed in the middle of the foot, therefore the boundary of the support polygon will be assumed to be symmetrical in either direction. Additionally, assuming that the vertical height of the COM is held constant, 6.5 represents an Ordinary Differential Equation (ODE) of the type

$$
\ddot{x}=a x+c,
$$


where $x$ is the time dependent variable, $a$ and $c$ are constants. This ODE has a general solution of the form

$$
x(t)=C_{1} \sinh (\sqrt{|a|} t)+C_{2} \cosh (\sqrt{|a|} t)+C_{3},
$$

where $C_{1}, C_{2}, C_{3}$ are constants dependent on the initial conditions. Applying this solution to 6.5 results in,

$$
x(t)=\dot{x}(0) \sqrt{\frac{y_{C O M}}{g}} \sinh \left(\sqrt{\frac{g}{y_{C O M}}} t\right)+\left( \pm x_{C O P}^{\max }+x(0)\right) \cosh \left(\sqrt{\frac{g}{y_{C O M}}} t\right)- \pm x_{C O P}^{\max } .
$$

This is a closed form solution of the open loop dynamics of the inverted pendulum model. This solution can be used to establish a stable phase plane region for the COM by applying the balance constraints

$$
-x_{C O P}^{\max }<x(t)<x_{C O P}^{\max }
$$

Inserting this constraint into 6.8 results in the following boundary on the COM phase plane

$$
\pm x_{C O P}^{\max }+x(0)+\dot{x}(0) \sqrt{\frac{y C O M}{g}}<0
$$

which can be rearranged as

$$
-x_{C O P}^{\max }<x(0)+\dot{x}(0) \sqrt{\frac{y_{C O M}}{g}}<x_{C O P}^{\max }
$$

This inequality defines a region in the COM phase plane in which the trajectory of the COM can be controlled to remain stable, as shown in Figure 6.1. Namely, the system can remain balanced by planning and tracking a reference COM trajectory that lies within the stable (green) region. However, once the COM exits the stable region, COM motion alone will be insufficient to maintain a balanced system and other strategies such as a stepping manoeuvre will be required $39,41,43,141]$.

\subsection{Push Disturbance Balancing Strategy Analysis}

In Section 6.1 a structure for analyzing the stability of the LIPM model was presented. This model can now be used to analyze how different behaviours affect the phase plane 


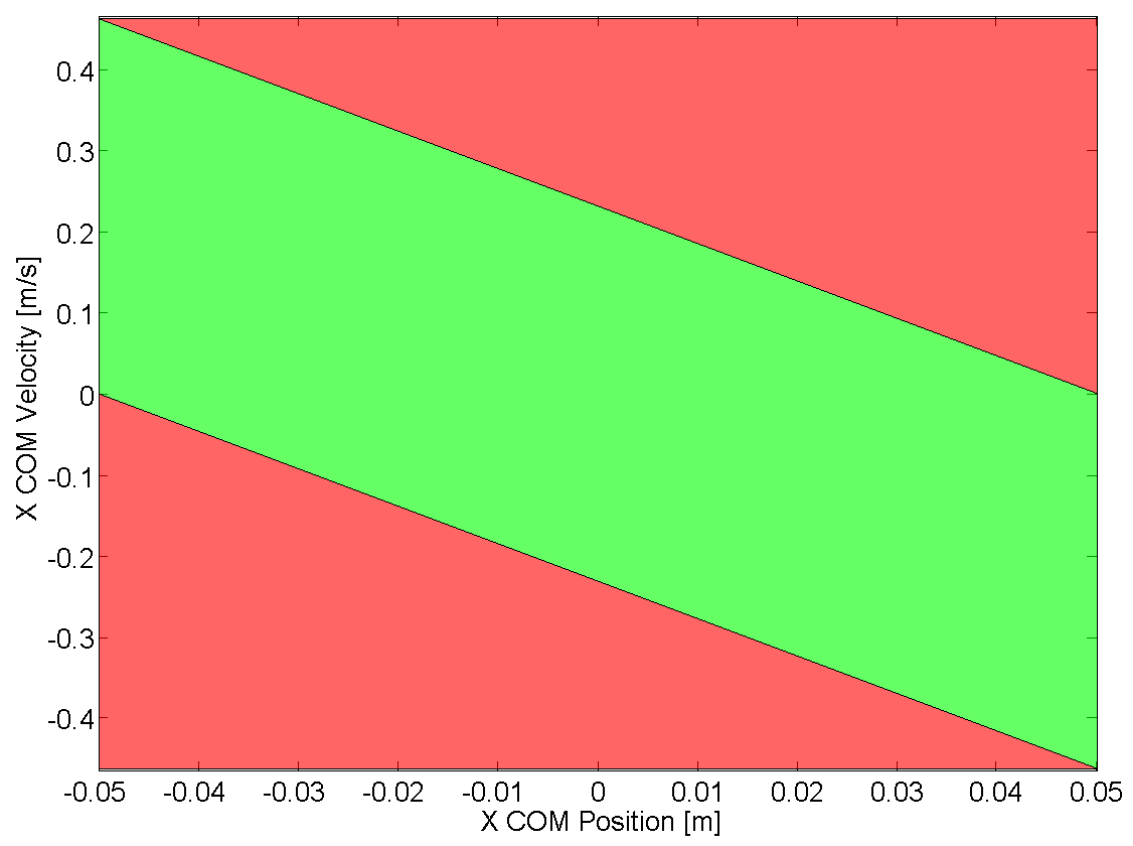

Figure 6.1: Stable region restriction on the $\mathrm{X}$ COM phase plane where the green region is stable and red is unstable.

stability region defined in 6.11). In this section, the push disturbance balancing strategy will be examined using this framework. From the results presented in Chapter 4, the BBLC

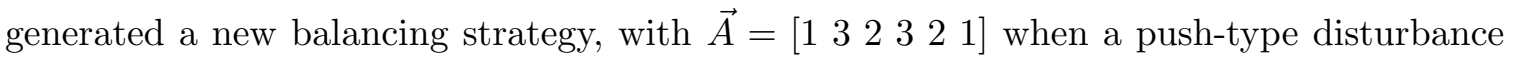
was applied. This balancing strategy primarily consisted of two behaviours: (1) activating CMP planning for the $\mathrm{X}$ and Z COM motion and (2) excluding the swing leg motion. In Sections 6.2.1 and 6.2.2, the effect of these behaviours on the phase plane stability region is examined. Then in Section 6.2.3, the expected stable regions are compared to the ones found from the experimental results.

\subsubsection{CMP Controller Effect on Stability}

The first key behaviour that is implemented in the push disturbance balancing strategy is planning the COM motion using the CMP controller defined in (4.4) and (4.5), where GRF feedback is used to modify the reference trajectory of the COM. 
First the change in the stability region defined in (6.11), as a result of applying a disturbance, can be examined. For this initial boundary, it was assumed that no external forces were applied to the system other than the GRFs, resulting in the simplifying assumption in (6.3). However, if a disturbance is applied then the simplifying assumption must be changed to

$$
F_{x}=m \ddot{x}_{C O M}+F_{x D},
$$

where $F_{x D}$ is the disturbance along the $\mathrm{X}$ axis. The resulting dynamic equation is

$$
\ddot{x}_{C O M}=\frac{g}{y_{C O M}}\left(x_{C O M}-x_{C O P}\right)-\frac{F_{x D}}{m} .
$$

with a closed form solution as

$x(t)=\dot{x}(0) \sqrt{\frac{y_{C O M}}{g}} \sinh \left(\sqrt{\frac{g}{y_{C O M}}} t\right)+\left( \pm x_{C O P}^{\max }+x(0)\right) \cosh \left(\sqrt{\frac{g}{y_{C O M}}} t\right) \pm x_{C O P}^{\max }-\frac{F_{x D} y_{C O M}}{m g}$.

This closed form solution results in the new phase plane stable region bounded by

$$
-x_{C O P}^{\max }-\frac{F_{x D} y_{C O M}}{m g}<x(0)+\dot{x}(0) \sqrt{\frac{y_{C O M}}{g}}<x_{C O P}^{\max }-\frac{F_{x D} y_{C O M}}{m g} .
$$

As shown in Figure 6.2, this causes the entire stable region to shift up or down along the $\dot{x}$ axis depending on the disturbance direction. In addition, the range of statically stable configurations is shifted. In the original region shown in Figure 6.1, the statically stable position of the COM, where $\dot{x}=0$, corresponds to the range of the support polygon, $-x_{C O P}^{\max }<x(t)<-x_{C O P}^{\max }$. When a disturbance is applied, this range is shifted by $-\frac{F_{x D} y_{C O M}}{m g}$. This indicates that there are now statically stable configurations where the position of the COM can lie outside of the support polygon and statically unstable configurations where the position of the COM lies within the support polygon.

Using a typical ZMP-based controller, the reference COM would still be planned to lie in the original stable region, given that there is no knowledge of the disturbance being applied. This may result in the controller planning a COM trajectory which lies in the unstable region when the disturbance is applied, as shown in Figure 6.2. 


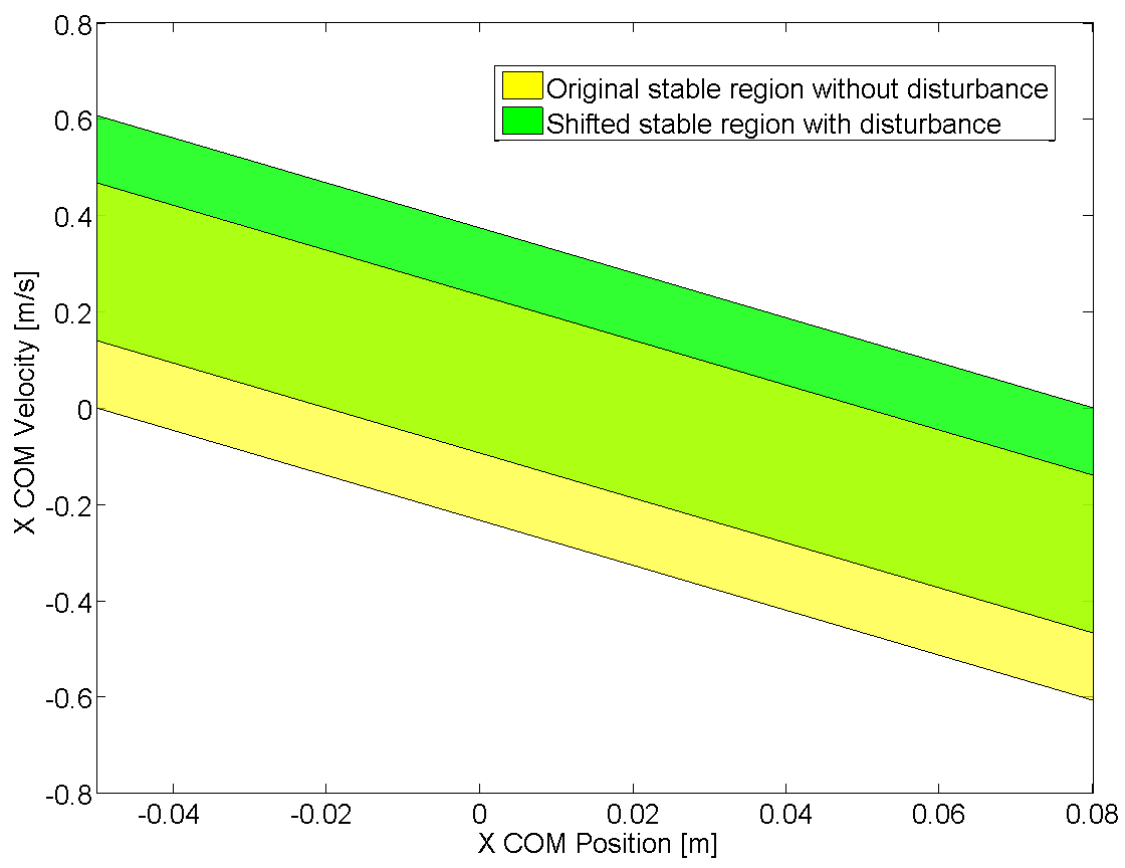

Figure 6.2: Stable region restriction on the $\mathrm{X}$ COM phase plane where the green region is stable and the yellow region corresponds to the undisturbed stable region.

Conversely, the CMP-based controller uses GRF force feedback to counteract this motion. We can substitute the reference COM controller defined in 4.4 into 6.15 resulting in a stable boundary for the CMP trajectory as

$$
-x_{C O P}^{\max }<x_{C M P}+\dot{x}_{C M P} \sqrt{\frac{y_{C O M}}{g}}<x_{C O P}^{\max } .
$$

This result shows that the CMP stable phase plane region is coincident with the original, undisturbed stable region defined in (6.11). If the CMP is planned within this region, the resulting reference COM will be planned in the stable region defined by (6.13), effectively accounting for the effect of the disturbance force applied to the system.

\subsubsection{Effect of Excluding Swing Leg Motion}

Qualitatively we can describe the effect of excluding the swing leg motion from motion planning as the control system focusing entirely on balance. Namely, the control system is no longer trying to walk, rather, it is using all joints to try and maintain balance. In the 
task priority architecture presented in Chapter 3, this results in the highest priority task to be removed from motion planning; no longer projecting lower priority tasks into the null space of the leg swing task-space Jacobian. This will result in the following change in the net projected Jacobians for the X and Z COM task-space motions from (3.5) as

$$
\begin{gathered}
J_{z C O M}^{\prime}=\left(I-J_{\text {swing }}^{\dagger} J_{\text {swing }}\right) J_{z C O M}, \\
J_{x C O M}^{\prime}=\left(I-J_{\text {swing }}^{\dagger} J_{\text {swing }}\right)\left(\left(I-J_{z C O M}^{\dagger} J_{z C O M}\right) J_{x C O M}\right),
\end{gathered}
$$

to

$$
\begin{gathered}
J_{z C O M}^{\prime}=J_{z C O M}, \\
J_{x C O M}^{\prime}=\left(I-J_{z C O M}^{\dagger} J_{z C O M}\right) J_{x C O M},
\end{gathered}
$$

where $J_{x C O M}$ and $J_{z C O M}$ are the Jacobians for the $\mathrm{X} \mathrm{COM}$ and $\mathrm{Z} \mathrm{COM}$ control and $J_{\text {swing }}$ is the Jacobian for swing leg control. This change in the projected Jacobian will have an effect on the manipulability of each Jacobian, affecting the maximum kinematically achievable COM velocity. Given that each Jacobian is defined for the motion of a single task-space motion $\left(X_{3} \in \mathbb{R}^{1}\right.$ and $\left.X_{5} \in \mathbb{R}^{1}\right)$, the maximum velocity is simply the principle Eigenvalue, $\sigma_{1}$, of the Jacobian 142 . This can be found by applying Singular Value Decomposition as

$$
J=U \sum V^{T}
$$

where $\sum$ will be a diagonal matrix composed of the Eigenvalues, $\sigma_{i} i=[1,2, \ldots, 13]$, of $\mathrm{J}$ and $U=\left[\vec{u}_{1}, \vec{u}_{2}, \ldots, \vec{u}_{13}\right]$. Note that for task-space motions where the dimensionality of $X_{r e f}>1$, a manipulability ellipsoid would be defined by the principal axes $\sigma_{1} \vec{u}_{1}$, $\sigma_{2} \vec{u}_{2}, \ldots, \sigma_{d_{i}} \vec{u}_{d_{i}}$. From the push disturbance case presented in Chapter 4, this maximum COM velocity is plotted in Figure 6.3 (a) and (b) for the $\mathrm{X}$ and $\mathrm{Z}$ axes respectively. From this figure, it can be seen that for both the $\mathrm{X}$ and $\mathrm{Z}$ COM motion, the maximum 

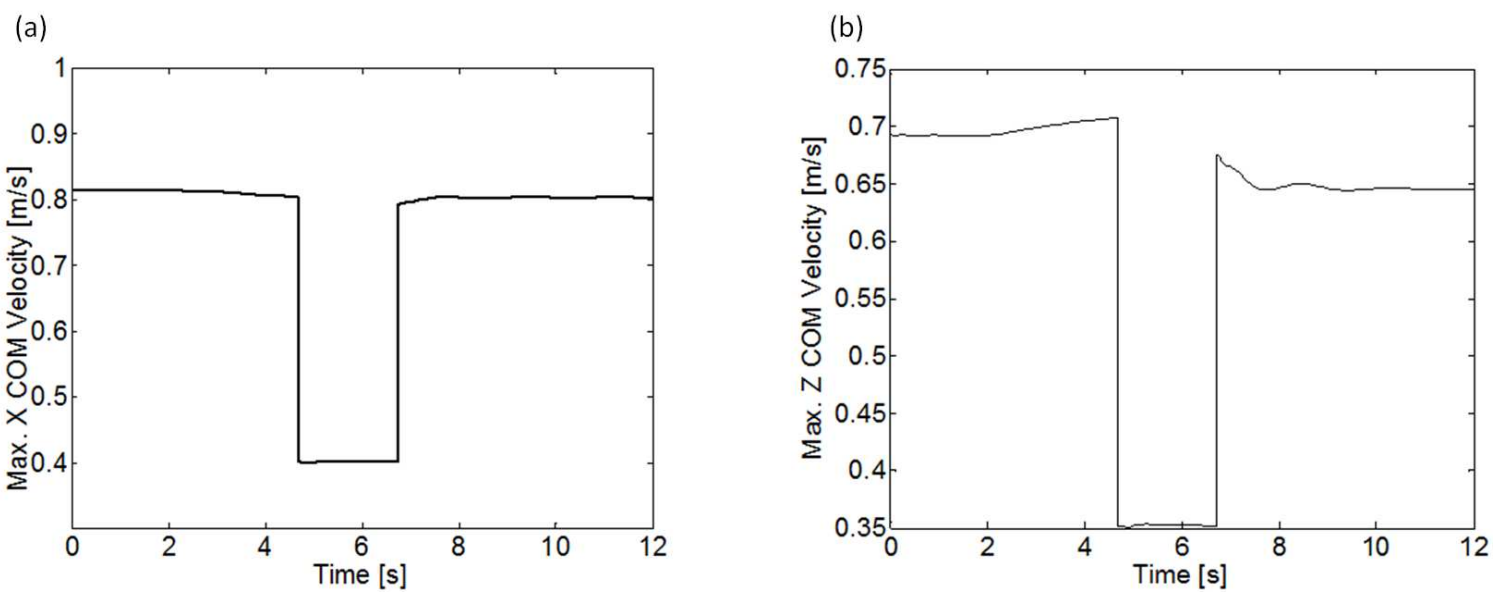

Figure 6.3: Maximum achievable COM velocity during push disturbance test as estimated by kinematic manipulability for (a) X COM and (b) Z COM. The manipulability is reduced during single stance, but returns to a higher level once the balancing strategy is engaged at $6.72 \mathrm{~s}$.

instantaneous velocity almost doubles from $0.4 \mathrm{~m} / \mathrm{s}$ to $0.8 \mathrm{~m} / \mathrm{s}$ in the $\mathrm{X}$ axis and 0.35 to 0.65 in the $\mathrm{Z}$ axis, when the balancing strategy is activated at $6.72 \mathrm{~s}$ and the swing leg motion is excluded from motion planning.

These velocity limits can be superimposed on the phase plane stability region for the COM found in Section 6.1 as shown in Figure 6.4. It can be seen that when the swing leg motion is not excluded, the maximum velocity constraint and the $\mathrm{X}$ and $\mathrm{Z}$ COM motions further reduce the stable region, when the LIPM is assumed to remain balanced. Conversely, when the swing leg motion is excluded, the COM velocity constraint lies outside the original stable boundary - increasing the reachable stable region for the control system. Therefore, the LIPM stability analysis shows how increasing the COM Jacobian's manipulability can increase the system's stability. This is also consistent with other works 143,144, where the effect of the COM Jacobian manipulability was related to balance control in ZMP-based controllers. 


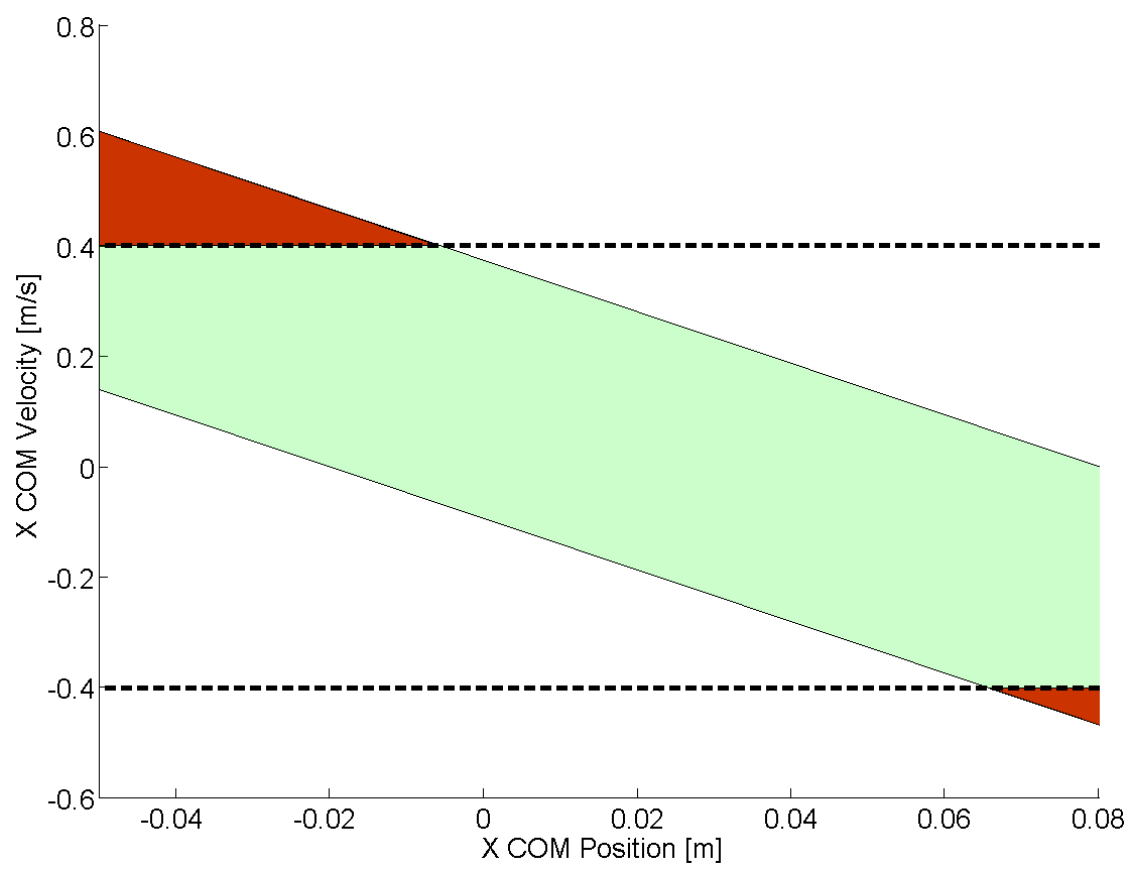

Figure 6.4: Stability region for X COM phase plane under $15 \mathrm{~N}$ push disturbance. Additional limits on $\mathrm{X}$ COM velocity result from manipulability of the COM, limiting the maximum achievable velocity.

\subsubsection{Results}

Given the stable regions defined by the analysis presented above, the results presented in Chapter 4 can now be analyzed within this framework. Figure 6.5 shows the phase plane Z COM motion for the push disturbance test where the disturbance is $15 \mathrm{~N}$ along the $\mathrm{X}$ axis and $25 \mathrm{~N}$ along the $\mathrm{Z}$ axis. In Figure 6.5, the reference trajectory for $\mathrm{Z} \mathrm{COM}$ is also plotted for both the CMP controller and default ZMP-based controller. It can be seen that the default trajectory lies outside of the stable region, while the CMP-based reference trajectory moves into the stable region, as the disturbance is applied, settling to $z_{C O M}=-0.03$ in steady state. This is consistent with the results, as for the same conditions, the default controller $\left(\vec{A}=\left[\begin{array}{llllll}1 & 1 & 1 & 1 & 1 & 1\end{array}\right]\right)$ results in a fall. Conversely, when the BBLC applies the balancing strategy with $\vec{A}=\left[\begin{array}{llllll}1 & 3 & 2 & 3 & 2 & 1\end{array}\right]$, the controller compensates for the disturbance and maintains balance. 
(a)
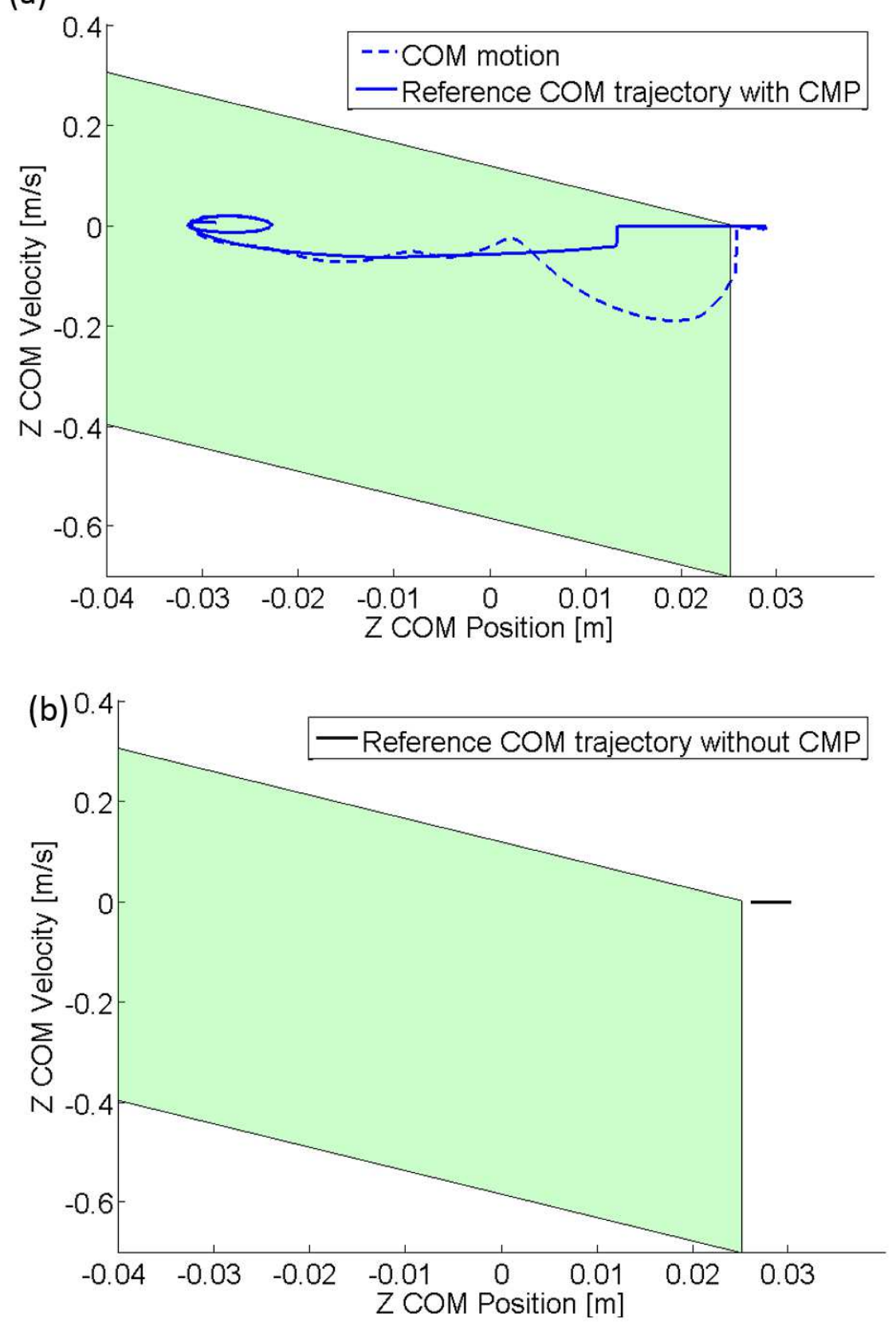

Figure 6.5: Phase plane trajectory of $\mathrm{Z} \mathrm{COM}$ when pushed by a $25 \mathrm{~N}$ disturbance superimposed on the expected stable region from the LIPM model. Without the CMP controller, the originally defined COM trajectory would lie outside the stable region. 


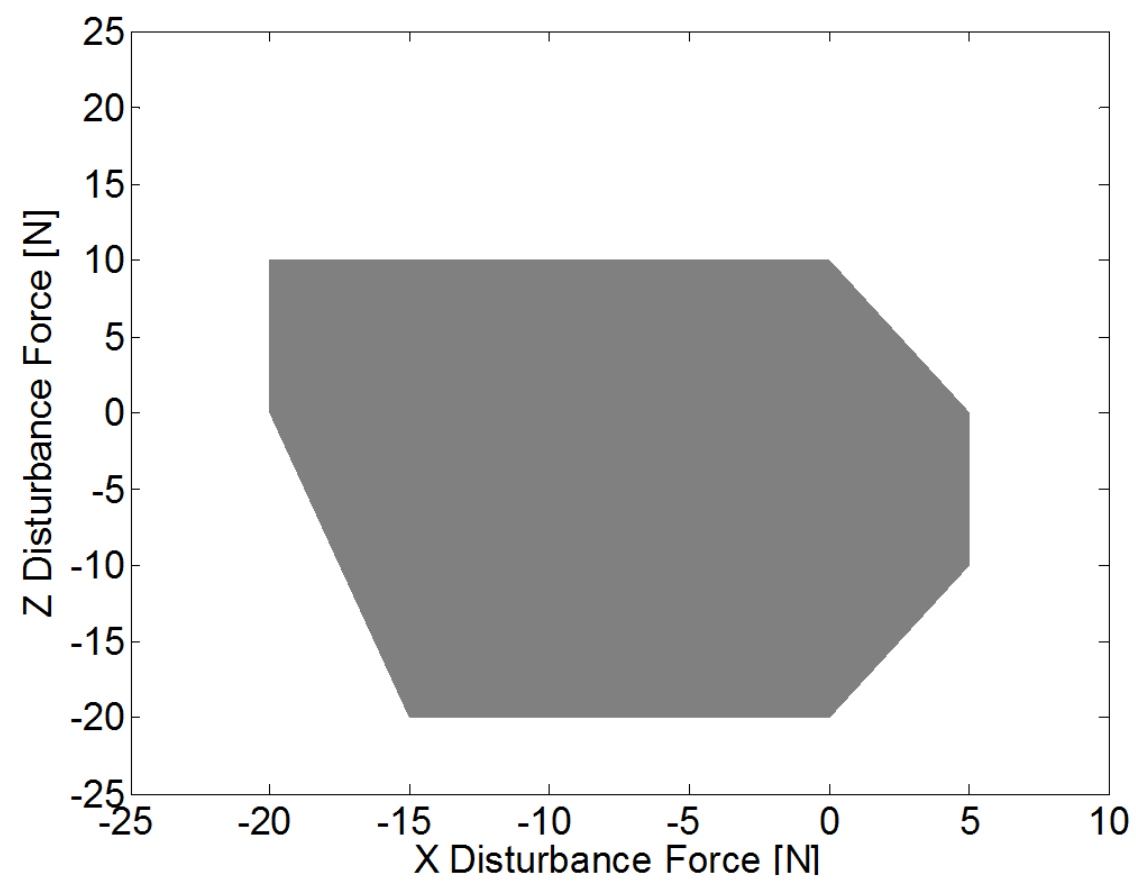

Figure 6.6: The shaded region corresponds to the region in which the default action vector

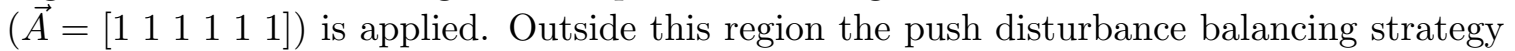

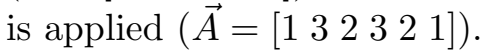

This result can be extended to estimate for which disturbances the default action vector

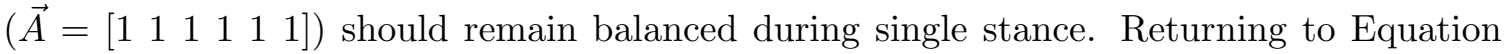
(6.15), the values $x(0)$ and $\dot{x}(0)$ can be assumed to be constant throughout single stance if $\vec{A}=\left[\begin{array}{llllll}1 & 1 & 1 & 1 & 1 & 1\end{array}\right]$. Given that all the other parameters are know, a maximum disturbance force can be calculated as

$$
F_{x D}=-\frac{\left(x(0) \pm x_{C O P}^{\max }\right) g m}{y_{C O M}}-\dot{x}(0) m .
$$

For the case examined above, the estimated maximum force in the $\mathrm{Z}$ axis is $22.6 \mathrm{~N}$ and 10 $\mathrm{N}$ in the $\mathrm{X}$ axis. In Figure 6.6, the region in which the balancing strategy is applied can be compared to the result from the analysis above. The shaded region corresponds to the region in which the default action vector continues to be applied when a disturbance is applied during single stance. Outside this region, the balancing strategy with $\vec{A}=\left[\begin{array}{llllll}1 & 3 & 2 & 3 & 2 & 1\end{array}\right]$, is applied to maintain balance of the robot. The upper boundaries for this region are 5 $\mathrm{N}$ for the $\mathrm{X}$ axis and $10 \mathrm{~N}$ for the $\mathrm{Z}$ axis. This result indicates the BBLC applies the 
balancing strategy for a threshold which is smaller than the one predicted by the LIPM analysis. Qualitatively, the controller can be described as being more conservative than is absolutely required to maintain balance. This difference can also be partially attributed to the discretization of the GRF data in the Q-table, which has a resolution of $5 \mathrm{~N}$. Overall, this result indicates that the BBLC successfully identifies the region in which a balancing behaviour needs to be applied; however, it may be overly conservative.

\subsection{Chapter Summary}

In this chapter, a stability analysis of the LIPM model was used to examine the effect of different balancing behaviour selections by the BBLC and their effect on stability. It was found that the balance constraints define a stable region in which the COM phase plane must remain to maintain balance. The effect of a push-type disturbance caused this stable region to shift. Additionally it was found that during single stance, the leg swing had the effect of reducing the size of the stable region by limiting the maximum achievable COM velocities by the controller.

By applying a CMP based planning strategy for the COM motion, and excluding the foot swing from the robot's motion control, the BBLC effectively accounts for the change in the stable phase plane region for the COM. This result shows that the learning algorithm of the BBLC is effectively identifying which regions in the state-space are unstable and applies the balancing strategies which can account for the changing balance constraints as disturbances are applied. 


\section{Chapter 7}

\section{Conclusions and Recommendations}

\subsection{Conclusions}

\subsubsection{BBLC Architecture}

Understanding bipedal balance is not only relevant to robotic locomotion, but also important in developing a better understanding of human walking and balance. Although this topic has been broadly studied, understanding what truly defines a balanced bipedal system remains ill-defined and has made controller design for bipedal robots challenging. This has limited most controllers for two legged robots to walking on flat level ground, with some special cases such as steps and slopes for which specific strategies have been developed. However, these controllers still lack the broader ability to walk on terrains where many different obstacles and disturbances may be present. The focus of the research presented in this thesis has been to develop a control architecture that can adapt to several different disturbances without any a priori planning. This was primarily achieved by applying a behaviour-based control architecture to the problem of maintaining balance. In this architecture several different balancing behaviours were combined together to generate new balancing strategies which can maintain balance when a disturbance is applied. To determine which strategies succeed for which disturbances, a learning algorithm is applied to iterate between different strategies and determine which ones are successful. 


\subsubsection{Simulation and Learning}

The controller was first implemented on two different platforms in simulation: a mobile manipulator and a planar biped. In both cases the BBLC was able to generate new balancing strategies when faced with various disturbances. These results confirmed the primary feature of the BBLC, that combining various balancing behaviours can result in emergent balancing strategies to unknown disturbances. The results also showed that the architecture was general enough that it could be implemented on two different robotic systems. Hence, the BBLC could be used anywhere balancing the system is a consideration and redundancy also exists in the system.

\subsubsection{Experimental Validation}

A more complete validation of the BBLC was then performed by implementing it on ABL-BI, both in simulation and in experiment. This represented a more challenging problem, given the added complexity of a 13-DOF bipedal robot as well as the challenges of an experimental platform. The initial simulation results showed that the BBLC was able to generate new balancing strategies for three distinct disturbances: a push disturbance, placing a step under one foot and walking up a slope. This confirmed the initial results from the planar biped implementation. In all cases, the results showed that applying the behaviour strategy resulted in an increase in the robustness of the system. The experiments validated these simulation results, where the same three disturbance cases were examined with ABL-BI. For all three cases, the BBLC applied the balancing strategies learnt in simulation, resulting in ABL-BI compensating for disturbances that would have otherwise caused a fall. This showed that the learning algorithm was model independent, given that the differences between the simulation and experimental platforms did not have an effect on the system performance. Finally, a more in-depth analysis of an emergent behaviour strategy was performed using the linearized inverted pendulum model. This analysis showed that the BBLC was effectively selecting balancing behaviours which compensated 
for the effect different disturbances had on the system's stable region.

Both the simulation and experimental results show that the BBLC provides a powerful control architecture where, through the combination of simple control behaviours, new balancing strategies can emerge to compensate for a wide range of disturbances. This is a departure from current approaches to robotic balance, where a single strategy is used or planned to compensate for a specific disturbances. This can allow robotic systems such as bipedal robots and exoskeletons to learn balancing strategies for a wide range of disturbances and allow for balanced walking on a much wider array of terrains than is currently possible with existing control systems.

\subsection{Recommendations for Future Work}

The work in this thesis presented the architecture of the BBLC as well as testing the controller with different disturbance cases. The key expansion for future work will be to increase the number of disturbances the BBLC learns to compensate for, and evaluating the controller's ability to walk in a highly unstructured environment. Overall, the following recommendations are made for future work:

- Increasing the number of behaviours available to the BBLC for bipedal locomotion. Principally including existing behaviours such as stepping manoeuvres and modelbased control approaches.

- Completing a more thorough analysis of the control architecture behaviours and priority scheme. This can include: the effect of switching between different behaviour combinations and a more complete analysis of how well the task-priority architecture allows multiple task-space motions to be tracked.

- An evaluation of the controller in a wider range of environments using multiple bipedal or humanoid platforms. This would involve a larger set of learning cases to determine 
if the BBLC can keep a bipedal walker balanced in a highly-unstructured environment, such as walking on rough terrain. 


\section{List of References}

[1] M. Hirose and K. Ogawa, "Honda humanoid robots development," Philosophical Transactions of the Royal Society A: Mathematical, Physical and Engineering Sciences, vol. 365, no. 1850, pp. 11-19, 2007.

[2] R. Zaier and S. Kanda, "Adaptive locomotion controller and reflex system for humanoid robots," 2008 IEEE/RSJ International Conference on Intelligent Robots and Systems, pp. 2492-2497, 2008.

[3] T. Ishida, Y. Kuroki, and J. Yamaguchi, "Mechanical system of a small biped entertainment robot," in IEEE/RSJ International Conference on Intelligent Robots and Systems, pp. 1129-1134, 2003.

[4] C. Chevallereau, G. Abba, Y. Aoustin, F. Plestan, E. Westervelt, C. Canudas-de Wit, and J. Grizzle, "RABBIT: A testbed for advanced control theory," IEEE Control Systems Magazine, vol. 23, no. 5, pp. 57-79, 2003.

[5] F. Pfeiffer, K. Loffler, and M. Gienger, "The concept of jogging johnnie," vol. 3, pp. 3129-3135, 2002.

[6] T. Sugihara, Y. Nakamura, and H. Inoue, "Real-time humanoid motion generation through ZMP manipulation based on inverted pendulum control," in IEEE International Conference on Robotics and Automation, pp. 1404-1409, 2002.

[7] J. Pratt, "Virtual Model Control: An Intuitive Approach for Bipedal Locomotion," The International Journal of Robotics Research, vol. 20, no. 2, pp. 129-143, 2001.

[8] E. Wayman, "Becoming human: The evolution of walking upright," Smithsonian.com, 2012.

[9] K. Hirai, M. Hirose, Y. Haikawa, and T. Takenaka, "The development of Honda humanoid robot," IEEE International Conference on Robotics and Automation, pp. 1321-1326, 1998.

[10] C. Chevallereau and P. Sardain, "Design and actuation optimization of a 4-axes biped robot for walking and running," in IEEE International Conference on Robotics and Automation. Symposia Proceedings, pp. 3365-3370, 2000. 
[11] S. Collins and A. Ruina, "A Bipedal Walking Robot with Efficient and Human-Like Gait," in IEEE International Conference on Robotics and Automation, pp. 1983-1988, 2005.

[12] B. Espiau and P. Sardain, "The anthropomorphic biped robot bip2000," IEEE Conference on Robotics and Automation, vol. 4, pp. 3996-4001, 2000.

[13] M. Gienger, K. Loffler, and F. Pfeiffer, "Towards the design of a biped jogging robot," in IEEE International Conference on Robotics and Automation, pp. 4140-4145, 2001.

[14] S. Shirata, A. Konno, and M. Uchiyama, "Design and development of a light-weight biped humanoid robot Saika-4," IEEE/RSJ International Conference on Intelligent Robots and Systems, pp. 148-153, 2004.

[15] B. Vanderborght, B. Verrelst, R. Van Ham, J. Naudet, J. Vermeulen, D. Lefeber, and F. Daerden, "LUCY, a Bipedal Walking Robot with Pneumatic Artificial Muscles," IEEE Mechatronics and Robotics Conference, 2004.

[16] S. Kajita, T. Nagasaki, K. Kaneko, and H. Hirukawa, "ZMP-Based Biped Running Control," IEEE Robotics \& Automation Magazine, vol. 14, no. 2, pp. 63-72, 2007.

[17] M. Vukobratovic and B. Borovac, "Zero-moment point-thirty five years of its life," International Journal of Humanoid Robotics, vol. 1, no. 1, pp. 157-173, 2004.

[18] H. Herr and M. B. Popovic, "Ground Reference Points in Legged Locomotion: Definitions, Biological Trajectories and Control Implications," The International Journal of Robotics Research, vol. 24, no. 12, pp. 1013-1032, 2005.

[19] S. Kajita, F. Kanehiro, K. Kaneko, K. Fujiwara, K. Harada, K. Yokoi, and H. Hirukawa, "Biped walking pattern generation by using preview control of zero-moment point," IEEE International Conference on Robotics and Automation, pp. 1620-1626, 2003.

[20] S. Kajita, O. Matsumoto, and M. Saigo, "Real-time 3D walking pattern generation for a biped robot with telescopic legs," IEEE International Conference on Robotics and Automation, pp. 2299-2306, 2001.

[21] T. Sugihara and Y. Nakamura, "A Fast Online Gait Planning with Boundary Condition Relaxation for Humanoid Robots," IEEE International Conference on Robotics and Automation, pp. 305-310, 2005.

[22] T. Sugihara, "Standing stabilizability and stepping maneuver in planar bipedalism based on the best COM-ZMP regulator," IEEE International Conference on Robotics and Automation, pp. 1966-1971, 2009. 
[23] T. Sugihara and Y. Nakamura, "Contact phase invariant control for humanoid robot based on variable impedant inverted pendulum model," IEEE International Conference on Robotics and Automation, pp. 51-56, 2003.

[24] J. J. Alcaraz-Jiménez, D. Herrero-Pérez, and H. Martínez-Barberá, "Robust feedback control of zmp-based gait for the humanoid robot nao," The International Journal of Robotics Research, vol. 32, no. 9-10, pp. 1074-1088, 2013.

[25] C. L. Shih and C. J. Chiou, "The motion control of a statically stable biped robot on an uneven floor.," IEEE transactions on systems, man, and cybernetics. Part B, Cybernetics, vol. 28, no. 2, pp. 244-9, 1998.

[26] C.-L. Shih, "Ascending and descending stairs for a biped robot," IEEE Transactions on Systems, Man, and Cybernetics - Part A: Systems and Humans, vol. 29, no. 3, pp. 255-268, 1999.

[27] S. Kajita, F. Kanehiro, K. Kaneko, K. Fujiwara, K. Harada, K. Yokoi, and H. Hirukawa, "Resolved momentum control: Humanoid motion planning based on the linear and angular momentum," in IEEE/RSJ International Conference on Intelligent Robots and Systems, 2003, vol. 2, pp. 1644-1650, 2003.

[28] S.-H. Lee and A. Goswami, "A momentum-based balance controller for humanoid robots on non-level and non-stationary ground," Autonomous Robots, vol. 33, no. 4, pp. 399-414, 2012.

[29] S.-H. Lee and A. Goswami, "Reaction Mass Pendulum (RMP): An explicit model for centroidal angular momentum of humanoid robots," IEEE International Conference on Robotics and Automation, pp. 4667-4672, 2007.

[30] A. Goswami, "Foot rotation indicator (FRI) point: a new gait planning tool to evaluate postural stability of biped robots," IEEE International Conference on Robotics and Automation, pp. 47-52, 1999.

[31] A. Goswami, "Postural Stability of Biped Robots and the Foot-Rotation Indicator (FRI) Point," The International Journal of Robotics Research, vol. 18, no. 6, pp. 523$533,1999$.

[32] A. Goswami and V. Kallem, "Rate of change of angular momentum and balance maintenance of biped robots," IEEE International Conference on Robotics and Automation, 2004, pp. 3785-3790, 2004.

[33] M. Popovic, A. Englehart, and H. Herr, "Angular momentum primitives for human walking: biomechanics and control," IEEE/RSJ International Conference on Intelligent Robots and Systems, pp. 1685-1691, 2004. 
[34] M. Popovic, A. Hofmann, and H. Herr, "Angular momentum regulation during human walking: biomechanics and control," IEEE International Conference on Robotics and Automation, pp. 2405-2411, 2004.

[35] M. Popovic, W. Gu, and H. Herr, "Conservation of angular momentum during human locomotion," Artificial Intelligence Laboratory Research Abstracts, pp. 264-265, 2002.

[36] H. Herr and M. Popovic, "Angular momentum in human walking.," The Journal of experimental biology, vol. 211, no. Pt 4, pp. 467-81, 2008.

[37] R. Beranek, H. Fung, and M. Ahmadi, "A Walking Stability Controller with Disturbance Rejection Based on CMP Criterion and Ground Reaction Force Feedback," in IEEE/RSJ International Conferance on Intelligent Robots and Systems, 2011.

[38] T. Koolen, T. de Boer, J. Rebula, A. Goswami, and J. Pratt, "Capturability-based analysis and control of legged locomotion, Part 1: Theory and application to three simple gait models," The International Journal of Robotics Research, vol. 31, no. 9, pp. 1094-1113, 2012.

[39] J. Pratt, J. Carff, S. Drakunov, and A. Goswami, "Capture Point: A Step toward Humanoid Push Recovery," IEEE-RAS International Conference on Humanoid Robots, pp. 200-207, 2006.

[40] J. R. Rebula, F. Canas, J. E. Pratt, and A. Goswami, "Learning Capture Points for Bipedal Push Recovery," IEEE International Conference on Robotics and Automation, pp. 1774-1774, 2008.

[41] B. Stephens, "Humanoid push recovery," IEEE-RAS International Conference on Humanoid Robots, pp. 589-595, 2007.

[42] B. J. Stephens and C. G. Atkeson, "Push recovery by stepping for humanoid robots with force controlled joints," in IEEE-RAS International Conference on Humanoid Robots, pp. 52-59, 2010.

[43] J. Pratt, T. Koolen, T. de Boer, J. Rebula, S. Cotton, J. Carff, M. Johnson, and P. Neuhaus, "Capturability-based analysis and control of legged locomotion, Part 2: Application to M2V2, a lower-body humanoid," The International Journal of Robotics Research, vol. 31, no. 10, pp. 1117-1133, 2012.

[44] R. C. Arkin, Behavior-Based Robotics . MIT Press, 1998.

[45] R. Brooks, "A robust layered control system for a mobile robot," IEEE Journal on Robotics and Automation, vol. 2, no. 1, pp. 14-23, 1986.

[46] M. Mataric and M. J. Matarić, "Behavior-based robotics as a tool for synthesis of artificial behavior and analysis of natural behavior.," Trends in cognitive sciences, vol. 2, no. 3, pp. 82-6, 1998 . 
[47] M. J. Mataric, "Behavior-based control: Main properties and implications," in IEEE International Conference on Robotics and Automation, Workshop on Architectures for Intelligent Control Systems, pp. 46-54, 1992.

[48] Deference Advanced Research Projects Agency, "About the robotics grand challenge," June 2014.

[49] B. Espiau and P. Sardain, "The anthropomorphic biped robot BIP2000," in IEEE Conference on Robotics and Automation, vol. 4, pp. 3996-4001, 2002.

[50] K. Hosoda, T. Takuma, and M. Ishikawa, "Design and control of a 3D biped robot actuated by antagonistic pairs of pneumatic muscles," in Proceedings of International Symposium on Adaptive Motion in Animals and Machines, 2005.

[51] K. Kaneko, F. Kanehiro, S. Kajita, H. Hirukawa, T. Kawasaki, M. Hirata, K. Akachi, and T. Isozumi, "Humanoid robot HRP-2," IEEE International Conference on Robotics and Automation, pp. 1083-1090, 2004.

[52] A. Konno, R. Sellaouti, F. Amar, and F. Ouezdou, "Design and development of the biped prototype ROBIAN," IEEE International Conference on Robotics and Automation, pp. 1384-1389, 2002.

[53] S. Lohmeier, T. Buschmann, H. Ulbrich, and F. Pfeiffer, "Modular joint design for performance enhanced humanoid robot LOLA," IEEE International Conference on Robotics and Automation, 2006, pp. 88-93, 2006.

[54] K. Nishiwaki, J. Kuffner, S. Kagami, M. Inaba, and H. Inoue, "The experimental humanoid robot H7: a research platform for autonomous behaviour.," Philosophical transactions. Series A, Mathematical, physical, and engineering sciences, vol. 365, no. 1850, pp. 79-107, 2007.

[55] N. Tsagarakis, M. Sinclair, F. Becchi, G. Metta, G. Sandini, and D. Caldwell, "Lower Body Design of the 'iCub' a Human-baby like Crawling Robot," IEEE-RAS International Conference on Humanoid Robots, pp. 450-455, 2006.

[56] B. Verrelst, R. Van Ham, B. Vanderborght, D. Lefeber, and F. Daerden, "Lucy: a walking biped,"

[57] M. Wisse and J. Frankenhuyzen, "Design and construction of MIKE; a 2-D autonomous biped based on passive dynamic walking," Adaptive Motion of Animals and Machines, pp. 143-154, 2006.

[58] T. Sugihara and Y. Nakamura, "Variable impedant inverted pendulum model control for a seamless contact phase transition on humanoid robot," in IEEE International Conference on Humanoid Robots, 2003. 
[59] T. Sugihara and Y. Nakamura, "Whole-body cooperative COG control through ZMP manipulation for humanoid robots," in 2nd Int. Symp. on Adaptive Motion of Animals and Machines, 2003.

[60] J.-I. Yamaguchi, A. Takanishi, and I. Kato, "Development of a biped walking robot compensating for three-axis moment by trunk motion," vol. 1, pp. 561-566, 1993.

[61] J. Yamaguchi, E. Soga, S. Inoue, and A. Takanishi, "Development of a bipedal humanoid robot-control method of whole body cooperative dynamic biped walking," in IEEE International Conference on Robotics and Automation, vol. 1, pp. 368-374, 2002.

[62] T. Sugihara and Y. Nakamura, "Whole-body cooperative balancing of humanoid robot using COG Jacobian," in IEEE/RSJ International Conference on Intelligent Robots and Systems, vol. 3, pp. 2575-2580, 2002.

[63] K. Nishiwaki and S. Kagami, "Online Walking Control System for Humanoids with Short Cycle Pattern Generation," The International Journal of Robotics Research, vol. 28, no. 6, pp. 729-742, 2009.

[64] J. H. Park and H. Chung, "ZMP compensation by online trajectory generation for biped robots," IEEE International Conference on Systems, Man, and Cybernetics, pp. 960-965, 1999.

[65] J. Strom, G. Slavov, and E. Chown, "Omnidirectional walking using zmp and preview control for the nao humanoid robot," RoboCup 2009: Robot Soccer World Cup XIII, pp. 378-389, 2010.

[66] Y. Kaneshima and A. Takanishi, "Online walking pattern generation for biped humanoid robot with trunk," IEEE International Conference on Robotics and Automation, pp. 3111-3116, 2002.

[67] I. Poulakakis and J. W. Grizzle, "The Spring Loaded Inverted Pendulum as the Hybrid Zero Dynamics of an Asymmetric Hopper," IEEE Transactions on Automatic Control, vol. 54, no. 8, pp. 1779-1793, 2009.

[68] T. Komura, H. Leung, and J. Kuffner, "A Feedback Controller for Biped Humanoids that Can Counteract Large Perturbations During Gait," IEEE International Conference on Robotics and Automation, pp. 1989-1995, 2005.

[69] G. A. Pratt, "Low Impedance Walking Robots," Integrative and Comparative Biology, vol. 42, no. 1, pp. 174-181, 2002.

[70] C. Atkeson and J. Morimoto, "Nonparametric representation of policies and value functions: A trajectory-based approach," Advances in neural information processing systems, pp. 1643-1650, 2003. 
[71] H. Benbrahim and J. A. J. Franklin, "Biped dynamic walking using reinforcement learning," Robotics and Autonomous Systems, vol. 22, no. 3-4, pp. 283-302, 1997.

[72] J. Braaksma, R. Babuska, and D. Hobbelen, "Reinforcement Learning Control for Biped Robot Walking on Uneven Surfaces," IEEE International Joint Conference on Neural Network Proceedings, pp. 4173-4178, 2006.

[73] G. Cheng, C. Atkeson, and G. Zeglin, "A simple reinforcement learning algorithm for biped walking," IEEE International Conference on Robotics and Automation, pp. 3030-3035, 2004.

[74] G. Cheng, C. Atkeson, and G. Zeglin, "Poincaré-Map-Based Reinforcement Learning For Biped Walking," IEEE International Conference on Robotics and Automation, pp. 2381-2386, 2005.

[75] C.-M. Chew and G. A. Pratt, "Dynamic bipedal walking assisted by learning," Robotica, vol. 20, no. 05, pp. 477-491, 2002.

[76] G. Endo, J. Morimoto, T. Matsubara, J. Nakanishi, and G. Cheng, "Learning CPGbased Biped Locomotion with a Policy Gradient Method: Application to a Humanoid Robot," The International Journal of Robotics Research, vol. 27, no. 2, pp. 213-228, 2008.

[77] T. Geng, "Fast Biped Walking with a Sensor-driven Neuronal Controller and Realtime Online Learning," The International Journal of Robotics Research, vol. 25, no. 3, pp. 243-259, 2006.

[78] T. Geng, B. Porr, and F. Wörgötter, "A reflexive neural network for dynamic biped walking control.," Neural computation, vol. 18, no. 5, pp. 1156-96, 2006.

[79] K. Hitomi, T. Shibata, Y. Nakamura, and S. Ishii, "Reinforcement learning for quasipassive dynamic walking of an unstable biped robot," Robotics and Autonomous Systems, vol. 54, no. 12, pp. 982-988, 2006.

[80] L. Hu, C. Zhou, and Z. Sun, "Estimating Probability Distribution with Q-learning for Biped Gait Generation and Optimization," IEEE/RSJ International Conference on Intelligent Robots and Systems, pp. 362-367, 2006.

[81] M. Huber and R. Grupen, "A control structure for learning locomotion gaits," in International Symposium on Robotic and Applications, 1998.

[82] D. Katić and M. Vukobratović, "Control Algorithm for Humanoid Walking Based on Fuzzy Reinforcement Learning," Serbian-Hungarian Joint Symposium on Intelligent Systems, pp. 81-93, 2006. 
[83] J. Kho and D. Lim, "A learning controller for repetitive gait control of biped walking robot," in SICE 2004 Annual Conference, vol. 1, pp. 885-889, 2004.

[84] A. Laud and G. DeJong, "Reinforcement learning and shaping: Encouraging intended behaviors," in International Conferene on Machine Learning, pp. 355-362, 2002.

[85] T. Mandersloot, M. Wisse, and C. Atkeson, "Controlling Velocity In Bipedal Walking: A Dynamic Programming Approach," IEEE-RAS International Conference on Humanoid Robots, pp. 124-130, 2006.

[86] P. Manoonpong, T. Geng, T. Kulvicius, B. Porr, and F. Wörgötter, "Adaptive, fast walking in a biped robot under neuronal control and learning.," PLoS Computational Biology, vol. 3, no. 7, p. e134, 2007.

[87] T. Matsubara, J. Morimoto, J. Nakanishi, M.-A. Sato, and K. Doya, "Learning CPGbased biped locomotion with a policy gradient method," IEEE-RAS International Conference on Humanoid Robots, pp. 208-213, 2005.

[88] T. Matsubara, M. Sato, and K. Doya, "Learning Sensory Feedback to CPG with Policy Gradient for Biped Locomotion," IEEE International Conference on Robotics and Automation, pp. 4164-4169, 2005.

[89] J. Morimoto and C. Atkeson, "Minimax differential dynamic programming: An application to robust biped walking," Advances in neural information processing systems, pp. 1563-1570, 2003.

[90] J. Morimoto, S. Hyon, G. Cheng, D. Bentivegna, and C. Atkeson, "Modulation of simple sinusoidal patterns by a coupled oscillator model for biped walking," IEEE International Conference on Robotics and Automation, 2006, pp. 1579-1584, 2006.

[91] J. Morimoto and C. Atkeson, "Learning Biped Locomotion," IEEE Robotics \& Automation Magazine, vol. 14, no. 2, pp. 41-51, 2007.

[92] J. Pratt and G. Pratt, "Stable adaptive control of a bipedal walking; robot with CMAC neural networks," IEEE International Conference on Robotics and Automation, pp. 1050-1056, 1999.

[93] M. Ogino, Y. Katoh, M. Aono, M. Asada, and K. Hosoda, "Reinforcement learning of humanoid rhythmic walking parameters based on visual information," Advanced Robotics, vol. 18, no. 7, pp. 677-697, 2004.

[94] A. W. Salatian, K. Y. Yi, and Y. F. Zheng, "Reinforcement learning for a biped robot to climb sloping surfaces," Journal of Robotic Systems, vol. 14, no. 4, pp. 283-296, 1997. 
[95] R. Tedrake, T. Zhang, and H. Seung, "Stochastic policy gradient reinforcement learning on a simple 3D biped," in IEEE/RSJ International Conference on Intelligent Robots and Systems, pp. 2849-2854, 2004.

[96] R. Tedrake, T. Zhang, and H. Seung, "Learning to walk in 20 minutes," in Yale Workshop on Adaptive and Learning Systems, 2005.

[97] T. Ueno, Y. Nakamura, T. Takuma, T. Shibata, K. Hosoda, and S. Ishii, "Fast and Stable Learning of Quasi-Passive Dynamic Walking by an Unstable Biped Robot Based on Off-Policy Natural Actor-Critic," IEEE/RSJ International Conference on Intelligent Robots and Systems, pp. 5226-5231, 2006.

[98] Y. Zhou and S. Member, "Dynamic fuzzy Q-learning control of uncertain systems with applications to humanoids," IEEE Conference on Control Applications, pp. 459-464, 2005.

[99] R. S. Sutton and A. G. Barto, Introduction to reinforcement learning. MIT Press, 1998.

[100] J. Shan and F. Nagashima, "Neural locomotion controller design and implementation for humanoid robot HOAP-1," in 20th Annual Conference of the Robotics Society of Japan, 2002.

[101] A. Sano and J. Furusho, "Realization of natural dynamic walking using the angular momentum information," in IEEE International Conference on Robotics and Automation, pp. 1476-1481, 2002.

[102] B. Stephens, Push recovery control for force-controlled humanoid robots. Carnegie Mellon University, 2011.

[103] H. Fung, Modelling, Simulation, and Control for a Bipedal Walking Robot by. Carleton Unversity, 2011.

[104] M. Carreras, J. Batlle, P. Ridao, and G. Roberts, "An overview on behaviour-based methods for AUV control," IFAC Conference on Manoeuvring and Control of Marine Crafts, 1999.

[105] M. Carreras, "PhD Thesis A Proposal of a Behavior-based Control Architecture with Reinforcement Learning for an Autonomous Underwater Robot," Universitat de Girona, 2004.

[106] R. Beranek, A. Morbi, and M. Ahmadi, "A Fuzzy Controller for Mobile Robots with Adjustable Length on Steep Sloping Terrains," in Canadan Society of Mechanical Engineers Conference, 2008. 
[107] G. Antonelli, F. Arrichiello, and S. Chiaverini, "The null-space-based behavioral control for autonomous robotic systems," Intelligent Service Robotics, vol. 1, no. 1, pp. 27-39, 2007.

[108] G. Antonelli, F. Arrichiello, and S. Chiaverini, "Stability analysis for the null-spacebased behavioral control for multi-robot systems," IEEE Conference on Decision and Control, pp. 2463-2468, 2008.

[109] Y. Koren and J. Borenstein, "Potential field methods and their inherent limitations for mobile robot navigation," in IEEE International Conference on Robotics and Automation, pp. 1398-1404, 1991.

[110] M. Proetzsch, T. Luksch, and K. Berns, "Development of complex robotic systems using the behavior-based control architecture ib2c," Robotics and Autonomous Systems, vol. 58 , no. 1 , pp. $46-67,2010$.

[111] J. Zhao and K. Berns, "Integrating capture point into biologically motivated controlled biped for maintaining stable state," in Adaptive Mobile Robotics: Proceedings of the 15th International Conference on Climbing and Walking Robots and the Support Technologies for Mobile Machines, pp. 255-262, 2012.

[112] M. Proetzsch, T. Luksch, and K. Berns, "The behaviour-based control architecture ib2c for complex robotic systems," in KI 2007: Advances in Artificial Intelligence, pp. 494-497, 2007.

[113] O. Barker, R. Beranek, and M. Ahmadi, "Design of a 13 Degree-of-Freedom Biped Robot with a CAN-Based Distributed Digital Control System," in Advanced Intelligent Mechatronics, pp. 836-841, 2010.

[114] A. Barbalace, A. Luchetta, G. Manduchi, M. Moro, A. Soppelsa, and C. Taliercio, "Performance comparison of vxworks, linux, rtai and xenomai in a hard real-time application," in IEEE-NPSS Real-Time Conference, pp. 1-5, 2007.

[115] J. Wang and Y. Li, "Kinematics and tip-over stability analysis for a mobile humanoid robot moving on a slope," IEEE International Conference on Automation and Logistics, pp. 2426-2431, 2008.

[116] Y. Nakamura, Advanced Robotics: Redundancy and Optimization . Addison Wesley, 1991.

[117] F. Arrichiello, S. Chiaverini, G. Indiveri, and P. Pedone, "The Null-Space-based Behavioral Control for Mobile Robots with Velocity Actuator Saturations," The International Journal of Robotics Research, vol. 29, no. 10, pp. 1317-1337, 2010. 
[118] G. Antonelli, F. Arrichiello, and S. Chiaverini, "The null-space-based behavioral control for autonomous robotic systems," Intelligent Service Robotics, vol. 1, no. 1, pp. 27-39, 2007.

[119] R. Zaier and F. Nagashima, "Motion Pattern Generator and Reflex System for Humanoid Robots," IEEE/RSJ International Conference on Intelligent Robots and Systems, pp. 840-845, 2006.

[120] R. Zaier and S. Kanda, "Piecewise-Linear Pattern Generator and Reflex System for Humanoid Robots," IEEE International Conference on Robotics and Automation, pp. 2188-2195, 2007.

[121] O. Hohn and W. Gerth, "Probabilistic Balance Monitoring for Bipedal Robots," The International Journal of Robotics Research, vol. 28, no. 2, pp. 245-256, 2009.

[122] S. Kalyanakrishnan and A. Goswami, "Learning to predict humanoid fall," International Journal of Humanoid Robotics, vol. 8, no. 02, pp. 245-273, 2011.

[123] C. J. C. H. Watkins and P. Dayan, "Q-learning," Machine Learning, vol. 22, no. 3-4, pp. 25-292, 1992.

[124] J. Peng and R. Williams, "Incremental multi-step Q-learning," Machine Learning, vol. 22, no. 1, pp. 283-290, 1996.

[125] S. Dubowsky and E. Vance, "Planning mobile manipulator motions considering vehicle dynamic stability constraints," in IEEE International Conference on Robotics and Automation, pp. 1271-1276, 1989.

[126] P. R. Roan, A. Burmeister, A. Rahimi, K. Holz, and D. Hooper, "Real-world validation of three tipover algorithms for mobile robots," in IEEE International Conference on Robotics and Automation, pp. 4431-4436, 2010.

[127] J. Kim, W. K. Chung, Y. Youm, and B. H. Lee, "Real-time zmp compensation method using motion for mobile manipulators," in IEEE International Conference on Robotics and Automation, pp. 1967-1972, 2002.

[128] A. M. Hootsmans, Norbert and S. Dubowsky, "Control of mobile manipulators including vehicle dynamics characteristics," Topical Meeting on Robotics and Remote Systems, 1991.

[129] Q. Huang, S. Sugano, and K. Tanie, "Motion planning for a mobile manipulator considering stability and task constraints," in IEEE International Conference on Robotics and Automation, pp. 2192-2198, 1998.

[130] Q. Huang, "Manipulator Motion Planning for Stabilizing a Mobile-Manipulator," IEEE/RSJ International Conference on Intelligent Robots and Systems, pp. 467-472, 1995. 
[131] D. A. Rey and E. G. Papadoupoulos, "Online automatic tipover prevention for mobile manipulators," IEEE/RSJ International Conference on Intelligent Robot and Systems, pp. 1273-1278, 1997.

[132] S. A. A. Moosavian and K. Alipour, "Moment-height tip-over measure for stability analysis of mobile robotic systems," in IEEE/RSJ International Conference on Intelligent Robots and Systems, pp. 5546-5551, 2006.

[133] A. Ghaffari, A. Meghdari, D. Naderi, and S. Eslami, "Tipover Stability Enhancement of Wheeled Mobile Manipulators Using an Adaptive Neuro-Fuzzy Inference Controller System," in Proceedings of World Academy of Science, Engineering and Technology, pp. 241-247, 2008.

[134] A. Meghdari, D. Naderi, and M. Alam, "Neural-network-based observer for real-time tipover estimation," Mechatronics, vol. 15, no. 8, pp. 989-1004, 2005.

[135] E. Papadopoulos and D. A. Rey, "A new measure of tipover stability margin for mobile manipulators," in IEEE International Conference on Robotics and Automation, vol. 4, pp. 3111-3116, 1996.

[136] A. De Luca, G. Oriolo, and C. Samson, "Feedback control of a nonholonomic car-like robot," in Robot motion planning and control, pp. 171-253, Springer, 1998.

[137] T. Sugihara, "Mobility Enhancement Control of Humanoid Robot based on Reaction Force Manipulation via Whole Body Motion," Ph.D. Thesis, University of Tokyo.

[138] J. Morimoto and C. Atkeson, "Learning Biped Locomotion," IEEE Robotics \& Automation Magazine, vol. 14, no. 2, pp. 41-51, 2007.

[139] P. Maes and R. Brooks, "Learning to coordinate behaviors," in Proceedings of the Eighth National Conference on Artificial Intelligence, pp. 796-802, 1990.

[140] G. Antonelli and F. Arrichiello, "Experiments of formation control with collisions avoidance using the Null-Space-based Behavioral control," Pace Pacing And Clinical Electrophysiology, 2006.

[141] J. Rebula, F. Canas, J. Pratt, and A. Goswami, "Learning Capture Points for humanoid push recovery," IEEE-RAS International Conference on Humanoid Robots, pp. 65-72, 2007.

[142] N. Naksuk and C. S. G. Lee, "Utilization of Movement Prioritization for Whole-Body Humanoid Robot Trajectory Generation," pp. 79-84, 2005.

[143] N. Naksuk and C. Lee, "Zero moment point manipulability ellipsoid," IEEE International Conference on Robotics and Automation, pp. 1970-1975, 2006. 
[144] S. Cotton, P. Fraisse, and A. P. Murray, "On the Manipulability of the Center of Mass of Humanoid Robots: Application to Design," Volume 2: 34th Annual Mechanisms and Robotics Conference, Parts $A$ and B, pp. 1259-1267, 2010. 


\section{Appendix A}

\section{Transformation Equations and Parameters for ABL-BI Kinematics}

Table A.1: Relative position between joint frames.

\begin{tabular}{l|r}
\hline Frame transformation & Position $[\mathrm{X}, \mathrm{Y}, \mathrm{Z}][\mathrm{m}]$ \\
\hline$\vec{P}_{0}^{1}$ & {$[-0.113,-0.017,-0.072]$} \\
$\vec{P}_{1}^{2}$ & {$[0,0,0]$} \\
$\vec{P}_{2}^{3}$ & {$[0,-0.12,0]$} \\
$\vec{P}_{3}^{4}$ & {$[-0.002,-0.18,0.0]$} \\
$\vec{P}_{4}^{5}$ & {$[0.01,-0.225,0.0]$} \\
$\vec{P}_{5}^{6}$ & {$[0.007,-0.025,0.07]$} \\
$\vec{P}_{0}^{7}$ & {$[0.113,-0.017,-0.072]$} \\
$\vec{P}_{7}^{8}$ & {$[0,0,0]$} \\
$\vec{P}_{8}^{9}$ & {$[0,-0.12,0]$} \\
$\vec{P}_{9}^{10}$ & {$[0.002,-0.18,0.0]$} \\
$\vec{P}_{10}^{11}$ & {$[-0.01,-0.225,0.0]$} \\
$\vec{P}_{11}^{12}$ & {$[-0.007,-0.025,0.07]$} \\
$\vec{P}_{0}^{13}$ & {$[0,0,0]$} \\
\hline
\end{tabular}


Table A.2: Relative position of the joint centre of mass to joint frame.

\begin{tabular}{|c|c|}
\hline Frame transformation & Position $[\mathrm{X}, \mathrm{Y}, \mathrm{Z}][\mathrm{m}]$ \\
\hline$\vec{P}_{0}^{0_{C O M}}$ & {$[0,0.0145,-0.136]$} \\
\hline$\vec{P}_{1}^{1_{C O M}}$ & {$[-0.002,-0.0238,0]$} \\
\hline$\vec{P}_{2}^{2}{ }_{C O M}$ & {$[-0.029,0.0035,0.0]$} \\
\hline$\vec{P}_{3}^{3{ }^{C O M}}$ & {$[-0.017,-0.146,0.0]$} \\
\hline$\vec{P}_{4}^{4}{ }_{C O M}$ & {$[-0.0095,-0.168,0.0]$} \\
\hline$\vec{P}_{5}^{5 C O M}$ & {$[0.012,-0.014,0.0001]$} \\
\hline$\vec{P}_{6}^{6_{C O M}}$ & {$[0,-0.004,0.009]$} \\
\hline$\vec{P}_{7}^{7} \mathrm{COM}$ & {$[0.002,-0.024,0.0]$} \\
\hline$\vec{P}_{8}^{8} \mathrm{COM}$ & {$[-0.029,0.0035,0.0]$} \\
\hline$\vec{P}_{9}^{9} \mathrm{COM}$ & {$[0.017,-0.146,0.0]$} \\
\hline$\vec{P}_{10}^{10_{C O M}}$ & {$[0.0095,-0.168,0.0]$} \\
\hline$\vec{P}_{11}^{11_{C O M}}$ & {$[0.012,-0.014,0.0001]$} \\
\hline$\vec{P}_{12}^{12}$ COM & {$[0,-0.004,0.009]$} \\
\hline$\vec{P}_{13}^{13_{C O M}}$ & {$[0,0.143,-0.131]$} \\
\hline
\end{tabular}

$T_{0}^{1}=\left[\begin{array}{cccc}\cos \left(\theta_{1}\right) & -\sin \left(\theta_{1}\right) & 0 & P_{0}^{1}(X) \\ \sin \left(\theta_{1}\right) & \cos \left(\theta_{1}\right) & 0 & P_{0}^{1}(Y) \\ 0 & 0 & 1 & P_{0}^{1}(Z) \\ 0 & 0 & 0 & 1\end{array}\right]$

$$
T_{1}^{2}=\left[\begin{array}{cccc}
\cos \left(\theta_{2}\right) & 0 & \sin \left(\theta_{2}\right) & P_{1}^{2}(X) \\
0 & 1 & 0 & P_{1}^{2}(Y) \\
-\sin \left(\theta_{2}\right) & 0 & \cos \left(\theta_{2}\right) & P_{1}^{2}(Z) \\
0 & 0 & 0 & 1
\end{array}\right]
$$




$$
\begin{aligned}
& T_{2}^{3}=\left[\begin{array}{cccc}
1 & 0 & 0 & P_{2}^{3}(X) \\
0 & \cos \left(\theta_{3}\right) & -\sin \left(\theta_{3}\right) & P_{2}^{3}(Y) \\
0 & \sin \left(\theta_{3}\right) & \cos \left(\theta_{3}\right) & P_{2}^{3}(Z) \\
0 & 0 & 0 & 1
\end{array}\right] \\
& T_{3}^{4}=\left[\begin{array}{cccc}
1 & 0 & 0 & P_{3}^{4}(X) \\
0 & \cos \left(\theta_{4}\right) & -\sin \left(\theta_{4}\right) & P_{3}^{4}(Y) \\
0 & \sin \left(\theta_{4}\right) & \cos \left(\theta_{4}\right) & P_{3}^{4}(Z) \\
0 & 0 & 0 & 1
\end{array}\right] \\
& T_{4}^{5}=\left[\begin{array}{cccc}
1 & 0 & 0 & P_{4}^{5}(X) \\
0 & \cos \left(\theta_{5}\right) & -\sin \left(\theta_{5}\right) & P_{4}^{5}(Y) \\
0 & \sin \left(\theta_{5}\right) & \cos \left(\theta_{5}\right) & P_{4}^{5}(Z) \\
0 & 0 & 0 & 1
\end{array}\right] \\
& T_{5}^{6}=\left[\begin{array}{cccc}
\cos \left(\theta_{6}\right) & -\sin \left(\theta_{6}\right) & 0 & P_{5}^{6}(X) \\
\sin \left(\theta_{6}\right) & \cos \left(\theta_{6}\right) & 0 & P_{5}^{6}(Y) \\
0 & 0 & 1 & P_{5}^{6}(Z) \\
0 & 0 & 0 & 1
\end{array}\right]
\end{aligned}
$$




$$
\begin{aligned}
& T_{0}^{7}=\left[\begin{array}{cccc}
\cos \left(\theta_{7}\right) & -\sin \left(\theta_{7}\right) & 0 & P_{0}^{7}(X) \\
\sin \left(\theta_{7}\right) & \cos \left(\theta_{7}\right) & 0 & P_{0}^{7}(Y) \\
0 & 0 & 1 & P_{0}^{7}(Z) \\
0 & 0 & 0 & 1
\end{array}\right] \\
& T_{7}^{8}=\left[\begin{array}{cccc}
\cos \left(\theta_{8}\right) & 0 & \sin \left(\theta_{8}\right) & P_{7}^{8}(X) \\
0 & 1 & 0 & P_{7}^{8}(Y) \\
-\sin \left(\theta_{8}\right) & 0 & \cos \left(\theta_{8}\right) & P_{7}^{8}(Z) \\
0 & 0 & 0 & 1
\end{array}\right] \\
& T_{8}^{9}=\left[\begin{array}{cccc}
1 & 0 & 0 & P_{8}^{9}(X) \\
0 & \cos \left(\theta_{9}\right) & -\sin \left(\theta_{9}\right) & P_{8}^{9}(Y) \\
0 & \sin \left(\theta_{9}\right) & \cos \left(\theta_{9}\right) & P_{8}^{9}(Z) \\
0 & 0 & 0 & 1
\end{array}\right] \\
& T_{8}^{9}=\left[\begin{array}{cccc}
1 & 0 & 0 & P_{9}^{10}(X) \\
0 & \cos \left(\theta_{10}\right) & -\sin \left(\theta_{10}\right) & P_{9}^{10}(Y) \\
0 & \sin \left(\theta_{10}\right) & \cos \left(\theta_{10}\right) & P_{9}^{10}(Z) \\
0 & 0 & 0 & 1
\end{array}\right]
\end{aligned}
$$




$$
\begin{aligned}
T_{10}^{11} & =\left[\begin{array}{cccc}
1 & 0 & 0 & P_{10}^{11}(X) \\
0 & \cos \left(\theta_{11}\right) & -\sin \left(\theta_{11}\right) & P_{10}^{11}(Y) \\
0 & \sin \left(\theta_{11}\right) & \cos \left(\theta_{11}\right) & P_{10}^{11}(Z) \\
0 & 0 & 0 & 1
\end{array}\right] \\
T_{11}^{12} & =\left[\begin{array}{cccc}
\cos \left(\theta_{12}\right) & -\sin \left(\theta_{12}\right) & 0 & P_{11}^{12}(X) \\
\sin \left(\theta_{12}\right) & \cos \left(\theta_{12}\right) & 0 & P_{11}^{12}(Y) \\
0 & 0 & 1 & P_{11}^{12}(Z) \\
0 & 0 & 0 & 1
\end{array}\right] \\
T_{0}^{13}= & {\left[\begin{array}{cccc}
13 \\
\cos \left(\theta_{13}\right) & -\sin \left(\theta_{13}\right) & 0 & P_{0}^{13}(X) \\
\sin \left(\theta_{13}\right) & \cos \left(\theta_{13}\right) & 0 & P_{0}^{13}(Y) \\
0 & 0 & 1 & P_{0}^{13}(Z) \\
0 & 0 & 0 & 1
\end{array}\right] }
\end{aligned}
$$


Appendix B

Calibration Curves for Ground Reaction Force Estimator 


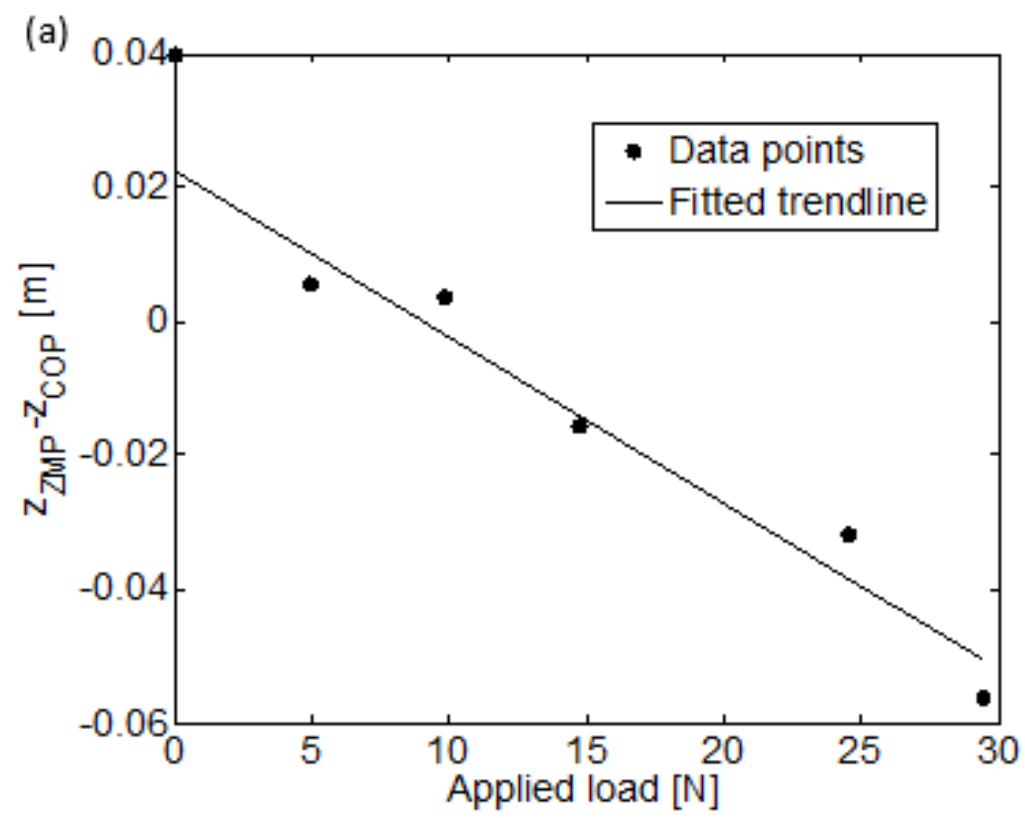

(b)

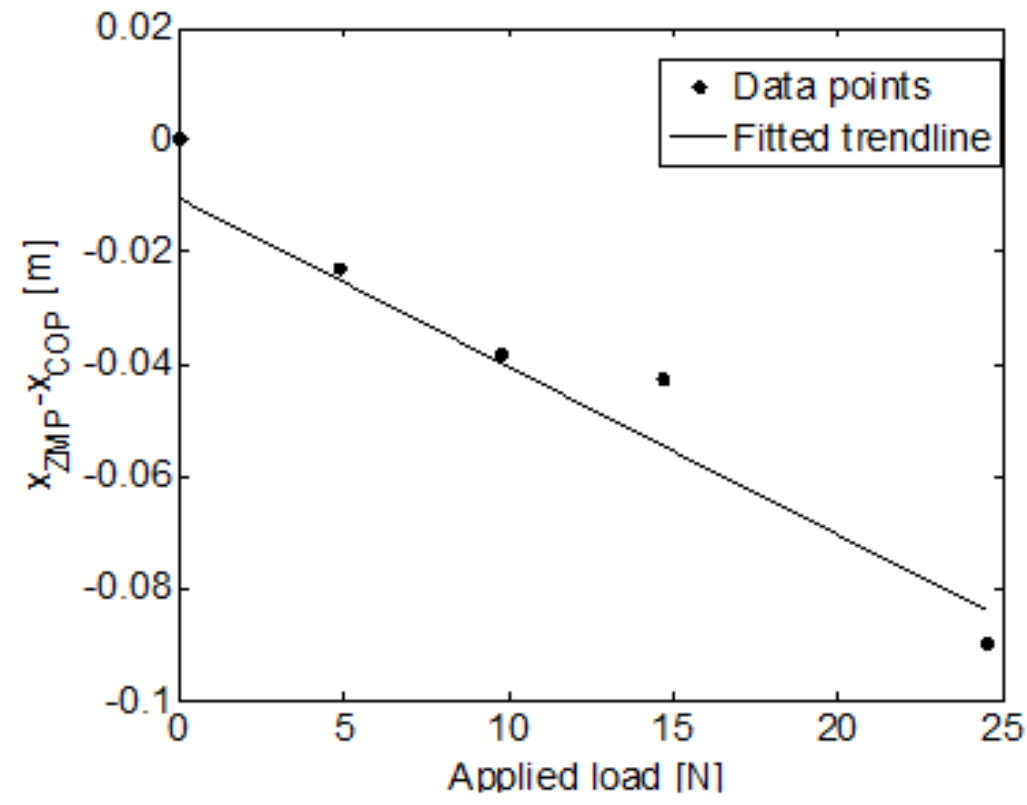

Figure B.1: Calibration curves used to establish the parameters for the ground reaction forces estimates. 